\title{
One-Pot Enantioselective Synthesis of 2-Pyrrolidinone Derivatives Bearing a Trifluoromethylated All-Carbon Quaternary Stereocenter
}

\author{
Zhuqing Jia, ${ }^{\dagger, \S}$ Xiaoyi Hu, ${ }^{\dagger, \S}$ Yunlong Zhao, ${ }^{\ddagger}$ Fayang G. Qiu, ${ }^{*}{ }^{\dagger}$ Albert S. C. Chan, ${ }^{*}{ }^{\star}$ and \\ Junling Zhao, \\ ${ }^{\dagger}$ Guangzhou Institutes of Biomedicine and Health, Chinese Academy of Sciences, Guangzhou 510530, \\ P. R. China. \\ Guangdong Provincial Key Laboratory of Chiral Molecule and Drug Discovery, School of \\ Pharmaceutical Sciences, Sun Yat-sen University, Guangzhou 510006, P. R. China. \\ ${ }^{\S}$ University of Chinese Academy of Sciences, Beijing 100049, P. R. China. \\ *E-mail: qiu_fayang@gibh.ac.cn; chenxz3@mail.sysu.edu.cn; zhaojling3@mail.sysu.edu.cn
}

\section{Table of contents}

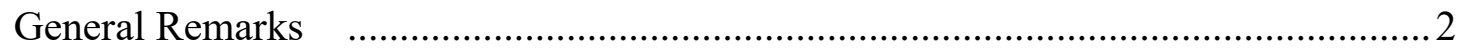

Typical procedures and characterization data for compounds 3 ...............................2

Typical procedures and characterization data for compounds $\mathbf{4 , 5}$ and $\mathbf{6} \ldots \ldots \ldots \ldots \ldots \ldots . . .54$

X-ray data of 3a 


\section{General Remarks}

All the addition reactions were carried out in the dried glassware under $\mathrm{N}_{2}$ atmosphere with magnetic stirring, while the reductive lactamizations were performed under $\mathrm{H}_{2}$ atmosphere. Reagents and organocatalysts were obtained from commercial supplier and used without further purification unless otherwise noted. NMR spectra were recorded on Bruker Avance $400 \mathrm{MHz}$ and $125 \mathrm{MHz}$ spectrometer. $\mathrm{CDCl}_{3}$ or DMSO-d6 were used as solvent. ${ }^{1} \mathrm{H}$ NMR spectra were referenced internally to the residual proton resonance in $\mathrm{CDCl}_{3}(\delta 7.26 \mathrm{ppm})$ and DMSO-d6 $(\delta$ $2.50 \mathrm{ppm})$. Chemical shifts $(\delta)$ reported as part per million $(\mathrm{ppm})$. 13C NMR spectra were referenced to $\mathrm{CDCl}_{3}(\delta 77.16 \mathrm{ppm}$, the middle peak) and DMSO-d6 $(\delta 39.52 \mathrm{ppm}$, the middle peak). Optical rotations were measured on a WZZ-2S Automatic polarimeter. High performance liquid chromatography was performed on Shimadzu LC-20AT Series HPLC using Daicel AD-H or OD-H chiral column eluted with a mixture of hexane and isopropyl alcohol. High resolution mass spectra were performed on an Agilent 6210 LC/MSD TOF spectrograph. X-ray structure analysis was carried out at an Oxford Gemini R Ultra diffractometer. Column chromatography was performed with silica gel (200-300 mesh), eluted with a mixture of petroleum ether and ethyl acetate.

Isatin-derived $\alpha$-trifluoromethylacrylates $\mathbf{1}$ were prepared according to our previously reported procedure. For details, see: Lou, Q.-X.; Ding, Y.-Y.; Xu, D.-F.; Liu, G.-K.; Zhao, J.-L. Adv. Synth. Catal. 2017, 359, 2557.

\section{Typical procedures and characterization data for compounds 3}

\section{General procedure for the synthesis of products 3}<smiles>[R]C([R])=C1C(=O)N(C(C)(C)C)C2=CC=[R1]#CC21</smiles>

1
$\mathrm{R}^{4} \mathrm{CH}_{2} \mathrm{NO}_{2} \underset{\text { 2) Raney } \mathrm{Ni} / \mathrm{H}_{2}, \mathrm{C}_{2} \mathrm{H}_{5} \mathrm{OH}, \mathrm{rt}}{\stackrel{\text { 28 }(10 \mathrm{~mol} \%), \text { neat, rt. }}{\longrightarrow}}$

2

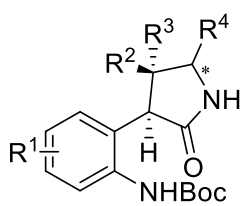

3

To a sample bottle equipped with a magnetic stirring bar, C8 (10 mol\%) and 2 ( $2 \mathrm{mmol}, 20.0$ equiv.) were stirred at room temperature under $\mathrm{N}_{2}$. Subsequently, $1(0.1 \mathrm{mmol}, 1.0$ equiv.) was added in one portion. After completion (about 3 to $6 \mathrm{~h}$, monitored by TLC), the reaction mixture was directly concentrated under reduced pressure. The residue was dissolved in $\mathrm{C}_{2} \mathrm{H}_{5} \mathrm{OH}(5.0 \mathrm{~mL})$, and Raney Nickel catalyst (10\%) was added, then the mixture was stirred at room temperature under hydrogen atmosphere until completion (about 10 to 30 hours, monitored by TLC). The reaction mixture was filtered through Celite. The filtrate was concentrated and the residue was purified by flash chromatography on silica gel (petroleum ether/ethyl acetate) to afford the compounds 3 .

General procedure for the synthesis of the racemic products 3 


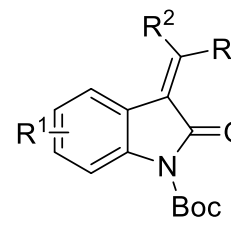

1

$$
\begin{gathered}
\text { 1) } \begin{array}{c}
\mathrm{DBU}, \mathrm{DCM} \\
\text { rt., } 0.5 \mathrm{~h}
\end{array} \\
\text { 2) Ranny Ni/H } \\
\mathrm{C}_{2} \mathrm{H}_{5} \mathrm{OH}, \mathrm{rt} .
\end{gathered}
$$

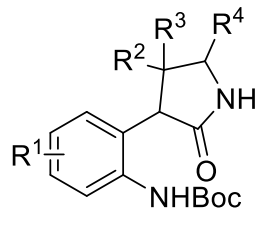

3

To a sample bottle equipped with a magnetic stirring bar, $1(0.1 \mathrm{mmol}, 1.0$ equiv. $)$ was added to a solution of DBU ( $0.01 \mathrm{mmol}, 0.1$ equiv.) and $2(1 \mathrm{mmol}, 10.0$ equiv. $)$ in DCM $(2.0 \mathrm{~mL})$ at room temperature under $\mathrm{N}_{2}$. After $0.5 \mathrm{~h}$, the reaction mixture was directly concentrated under reduced pressure. The residue was dissolved in $\mathrm{C}_{2} \mathrm{H}_{5} \mathrm{OH}(5.0 \mathrm{~mL})$, and Raney Nickel catalyst $(10 \%)$ was added, then the mixture was stirred at room temperature under hydrogen atmosphere until completion (about 10 to 30 hours, monitored by TLC). The reaction mixture was filtered through Celite. The filtrate was concentrated and the residue was purified by flash chromatography on silica gel (petroleum ether/ethyl acetate) to afford the racemic compounds 3 . 
ethyl (3S,4S)-4-(2-((tert-butoxycarbonyl) amino) phenyl)-5-oxo-3-(trifluoromethyl) pyrrolidine-3-carboxylate (3a)<smiles>CCOC(=O)c1ccccc1Nc1ccccc1C1C(=O)NCC1(C(F)(F)F)C(F)(F)F</smiles>

3a

3a was obtained as a white foam in 95\% yield ( $3 \mathrm{~h}$ for Michael addition and $10 \mathrm{~h}$ for catalytic hydrogenation), $96 \%$ ee and $>20: 1 \mathrm{dr}$. The enantiomeric excess was determined by HPLC (Daicel Chiralpak AD-H, hexane/i-PrOH $=80: 20(\mathrm{v} / \mathrm{v}), \lambda=254 \mathrm{~nm}$, flow rate $\left.=1.0 \mathrm{~mL} / \mathrm{min}, 25^{\circ} \mathrm{C}\right): t_{\text {minor }}=4.89 \mathrm{~min}$, $t_{\text {major }}=3.78 \mathrm{~min} ;[\alpha]_{\mathrm{D}}{ }^{25} 11.3(c 0.33, \mathrm{MeOH}) ;{ }^{1} \mathrm{H} \mathrm{NMR}\left(400 \mathrm{MHz}, \mathrm{DMSO}-d_{6}\right)$ $\delta 8.75(\mathrm{~s}, 1 \mathrm{H}), 8.48(\mathrm{~s}, 1 \mathrm{H}), 7.38(\mathrm{~d}, J=8.0 \mathrm{~Hz}, 1 \mathrm{H}), 7.25(\mathrm{t}, \mathrm{J}=7.6 \mathrm{~Hz}, 1 \mathrm{H})$, $7.08(\mathrm{t}, J=7.6 \mathrm{~Hz}, 1 \mathrm{H}), 6.92(\mathrm{~d}, J=7.7 \mathrm{~Hz}, 1 \mathrm{H}), 4.69(\mathrm{~s}, 1 \mathrm{H}), 4.04(\mathrm{~d}, J=11.7 \mathrm{~Hz}, 1 \mathrm{H}), 3.75(\mathrm{dq}$, $J=10.6,7.1 \mathrm{~Hz}, 1 \mathrm{H}), 3.70-3.52(\mathrm{~m}, 2 \mathrm{H}), 1.45(\mathrm{~s}, 9 \mathrm{H}), 0.80(\mathrm{t}, J=7.1 \mathrm{~Hz}, 3 \mathrm{H}) \mathrm{ppm} .{ }^{13} \mathrm{C}$ NMR $\left(125 \mathrm{MHz}, \mathrm{DMSO}-d_{6}\right) \delta 173.46,165.69,154.05,138.48,129.33,128.54,128.49,126.17,126.11$ (q, $J=282.5 \mathrm{~Hz}), 124.78,79.05,62.58,59.55$ (q, $J=25.4 \mathrm{~Hz}), 45.44,43.93,28.54,13.41 \mathrm{ppm}$; ${ }^{19} \mathrm{~F}$ NMR (471 MHz, DMSO- $\left.d_{6}\right) \delta-71.50 \mathrm{ppm}$. ESI-HRMS m/z: $417.1638[\mathrm{M}+\mathrm{H}]{ }^{+}$, $\mathrm{C}_{19} \mathrm{H}_{23} \mathrm{~F}_{3} \mathrm{~N}_{2} \mathrm{O}_{5}+\mathrm{H}^{+}$requires 417.1632.
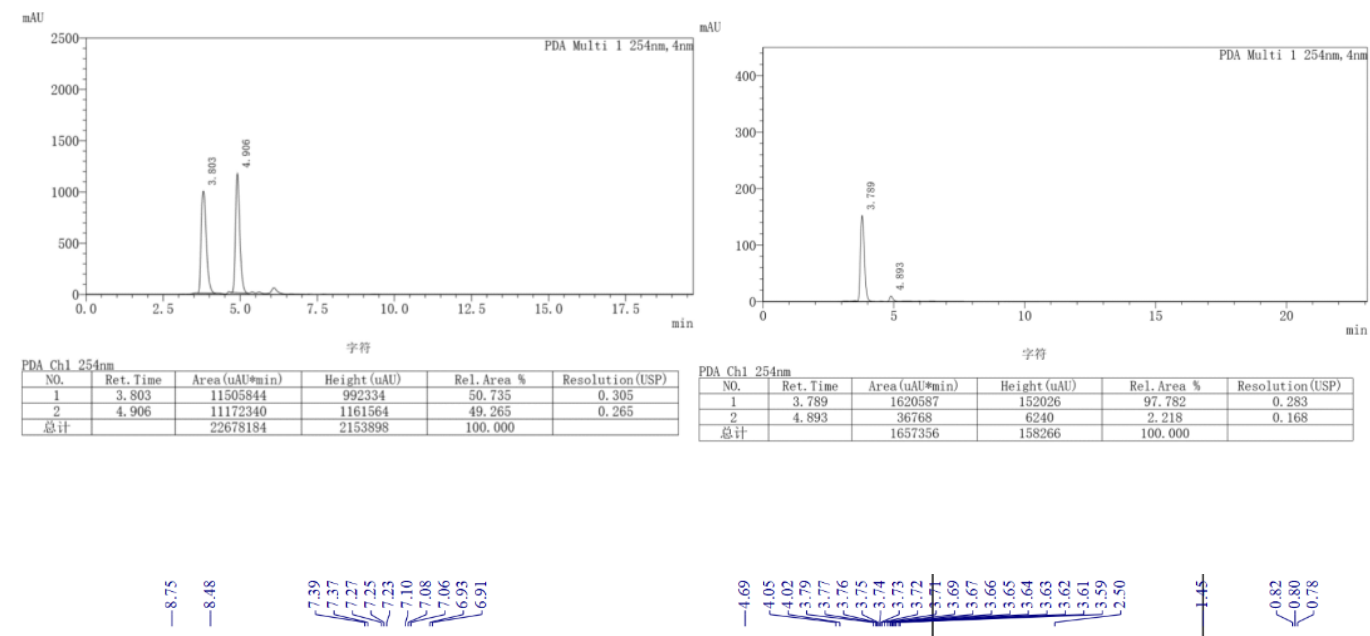<smiles>CCOC(=O)C(C)(C)C1CNC(=O)[C@H]1c1ccccc1N</smiles>

3a
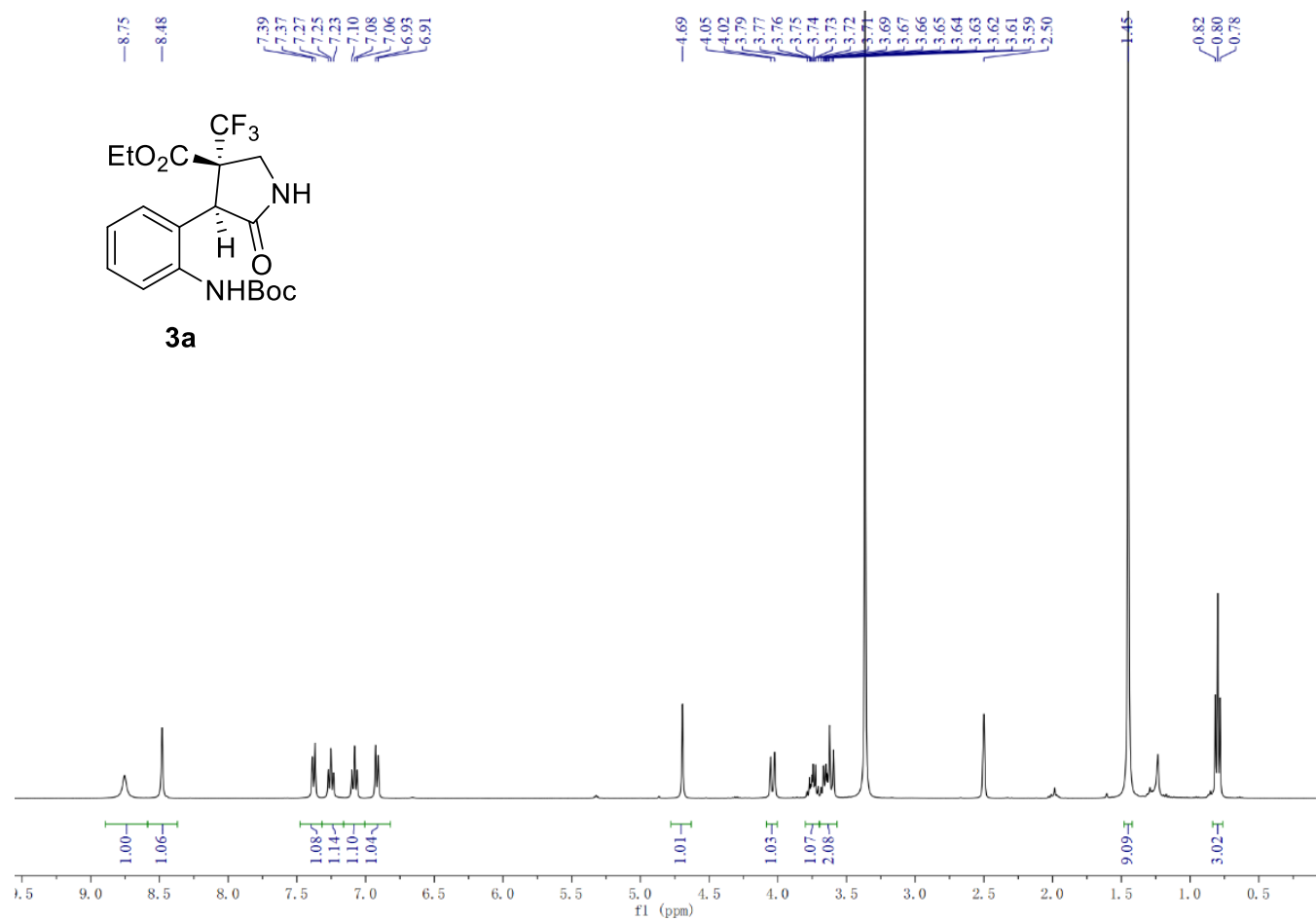

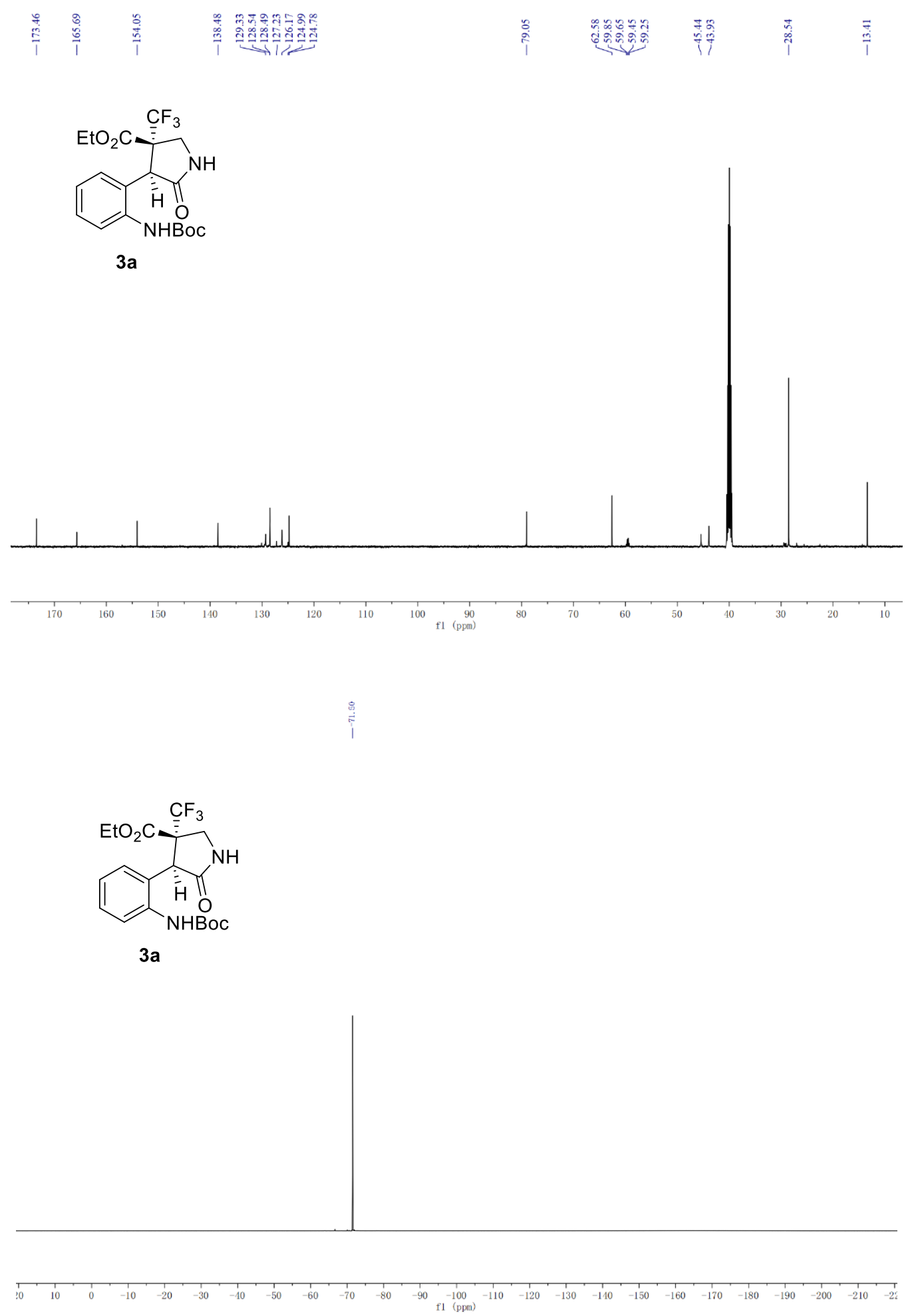
<smiles>CCOC(=O)c1cc(C)ccc1N=C1NCC(C(C)(C)C)(C(F)(F)F)C1c1cccc(C)c1</smiles>

$3 b$

3b was obtained as a yellow foam in $87 \%$ yield ( $4 \mathrm{~h}$ for Michael addition and $12 \mathrm{~h}$ for Catalytic hydrogenation), $91 \%$ ee and > 20:1 dr. The enantiomeric excess was determined by HPLC (Daicel Chiralpak AD-H, hexane/i-PrOH $=80: 20(\mathrm{v} / \mathrm{v}), \lambda=254 \mathrm{~nm}$, flow rate $\left.=1.0 \mathrm{~mL} / \mathrm{min}, 25^{\circ} \mathrm{C}\right)$ : $t_{\text {minor }}=5.91 \mathrm{~min}, t_{\text {major }}=3.75 \mathrm{~min} ;[\alpha]_{\mathrm{D}}{ }^{25} 52.3(c 0.47, \mathrm{MeOH}) ;{ }^{1} \mathrm{H} \mathrm{NMR}$ (500 MHz, Chloroform- $d$ ) $\delta 7.61(\mathrm{~s}, 1 \mathrm{H}), 7.47(\mathrm{~s}, 1 \mathrm{H}), 7.09(\mathrm{~d}, J=8.3 \mathrm{~Hz}$, $1 \mathrm{H}), 6.83(\mathrm{~s}, 1 \mathrm{H}), 6.63(\mathrm{~d}, J=8.3 \mathrm{~Hz}, 1 \mathrm{H}), 4.28(\mathrm{~d}, J=12.2 \mathrm{~Hz}, 2 \mathrm{H}), 3.85$ (dq, $J=14.2,7.4,5.6 \mathrm{~Hz}, 1 \mathrm{H}), 3.73(\mathrm{td}, J=13.1,12.1,6.8 \mathrm{~Hz}, 2 \mathrm{H}), 2.27(\mathrm{~s}, 3 \mathrm{H}), 1.50(\mathrm{~s}, 9 \mathrm{H})$, $0.89(\mathrm{t}, J=7.2 \mathrm{~Hz}, 3 \mathrm{H}) \mathrm{ppm} .{ }^{13} \mathrm{C}$ NMR $(125 \mathrm{MHz}$, Chloroform- $d$ ) $\delta 174.85,165.18,153.60$, $135.11,134.65,129.82,127.02,125.96,125.61(\mathrm{q}, J=283.1 \mathrm{~Hz}), 122.22,80.34,62.98,59.64(\mathrm{q}$, $J=25.9,25.3 \mathrm{~Hz}), 46.12,44.16,28.30,20.87,13.14 \mathrm{ppm} ;{ }^{19} \mathrm{~F}$ NMR (471 MHz, Chloroform- $d$ ) $\delta$ -73.46 ppm. ESI-HRMS m/z: $431.1785[\mathrm{M}+\mathrm{H}]{ }^{+}, \mathrm{C}_{20} \mathrm{H}_{25} \mathrm{~F}_{3} \mathrm{~N}_{2} \mathrm{O}_{5}+\mathrm{H}^{+}$requires 431.1788.
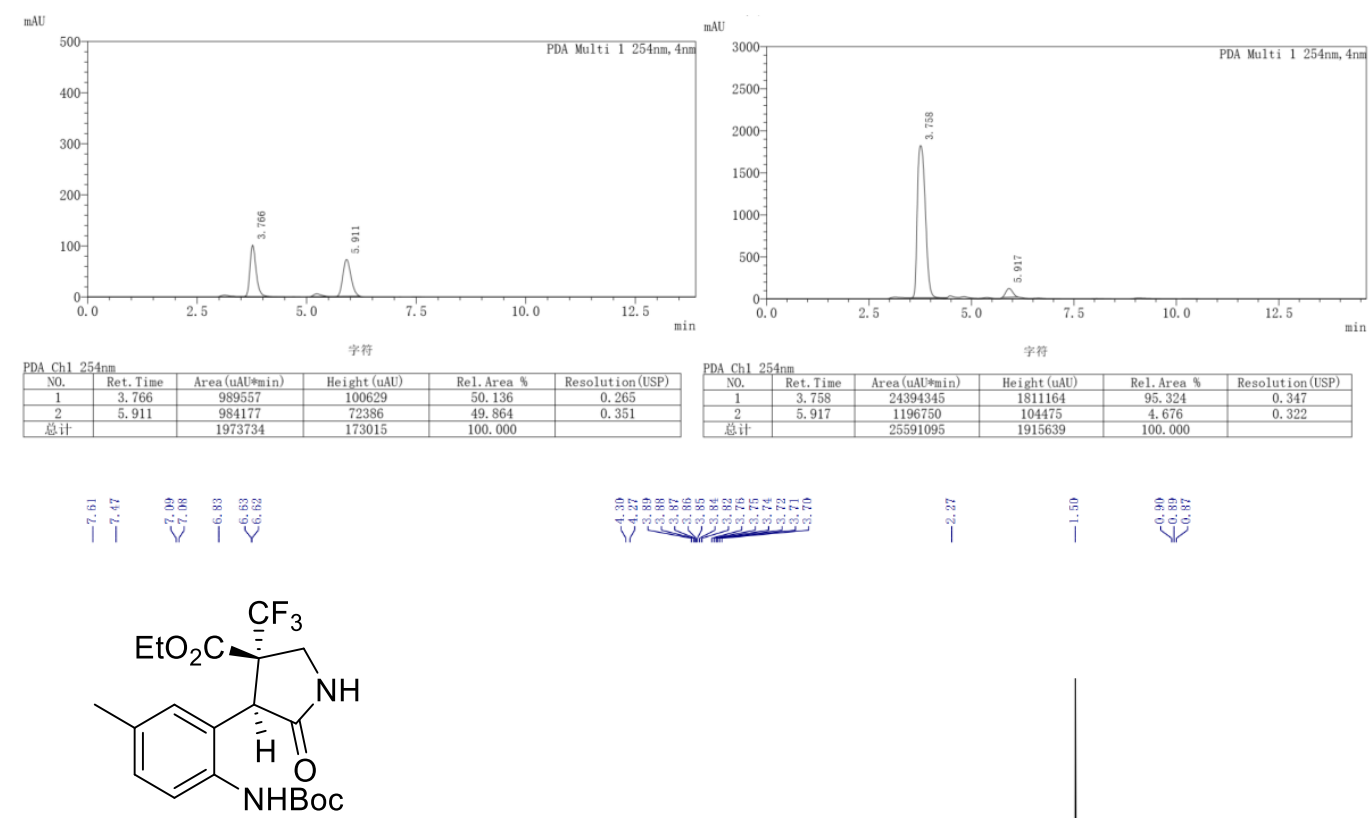

3b

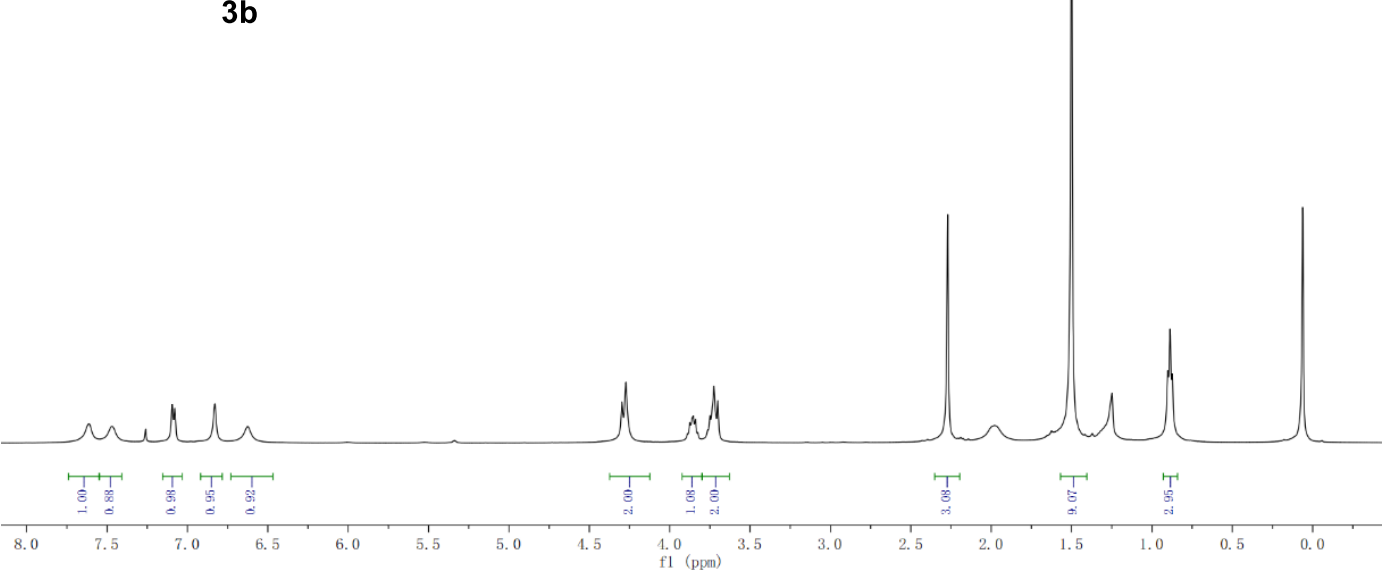




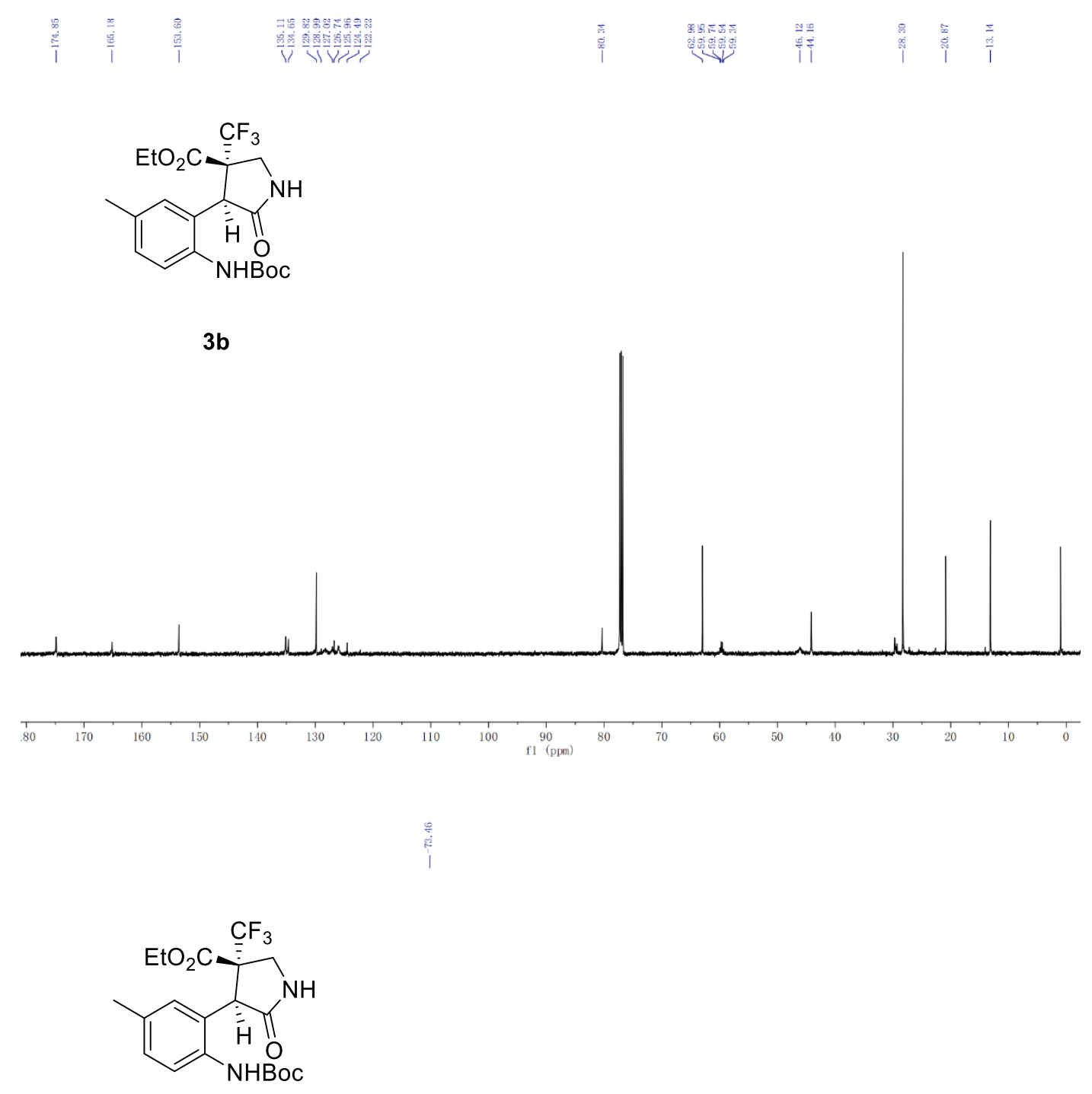

$3 \mathbf{b}$

ethyl (3S,4S)-4-(2-((tert-butoxycarbonyl) amino)-5-methoxyphenyl)-5-oxo-3-(trifluoromethyl) pyrrolidine-3-carboxylate (3c) 
<smiles>CCOC(=O)Nc1ccc(OC)cc1C1C(=O)NCC1(C(F)(F)F)C(F)(F)F</smiles>

$3 c$

3c was obtained as a white foam in $93 \%$ yield ( $4 \mathrm{~h}$ for Michael addition and $12 \mathrm{~h}$ for catalytic hydrogenation), 94\% ee and > 20:1 dr. The enantiomeric excess was determined by HPLC (Daicel Chiralpak AD-H, hexane $/ \mathrm{i}-\mathrm{PrOH}=80: 20(\mathrm{v} / \mathrm{v}), \lambda=254 \mathrm{~nm}$, flow rate $\left.=1.0 \mathrm{~mL} / \mathrm{min}, 25^{\circ} \mathrm{C}\right)$ : $t_{\text {minor }}=5.80 \mathrm{~min}, t_{\text {major }}=4.23 \mathrm{~min} ;[\alpha]_{\mathrm{D}}{ }^{25}-7.5(c 0.45, \mathrm{MeOH}) ;{ }^{1} \mathrm{H} \mathrm{NMR}$

(400 MHz, Chloroform-d) $\delta 7.46$ (s, 1H), 7.38 (s, 1H), 6.83 (dd, J = 8.9, $2.9 \mathrm{~Hz}, 1 \mathrm{H}), 6.65-6.40(\mathrm{~m}, 2 \mathrm{H}), 4.29$ (d, $J=11.4 \mathrm{~Hz}, 2 \mathrm{H}), 3.90(\mathrm{dq}, \mathrm{J}=10.6,7.1 \mathrm{~Hz}, 1 \mathrm{H}), 3.84-$ $3.59(\mathrm{~m}, 5 \mathrm{H}), 1.50(\mathrm{~s}, 9 \mathrm{H}), 0.92(\mathrm{t}, J=7.2 \mathrm{~Hz}, 3 \mathrm{H}) \mathrm{ppm} .{ }^{13} \mathrm{C}$ NMR $(125 \mathrm{MHz}$, Chloroform- $d) \delta$ 173.51, 164.19, 156.19, 152.88, 129.08, 128.90, 125.66, 123.71 (q, $J=283.7 \mathrm{~Hz}), 123.41,113.08$, 79.27, 62.05, $58.51(\mathrm{q}, J=26.0 \mathrm{~Hz}), 54.50,45.26,43.14,27.30,12.21 \mathrm{ppm} ;{ }^{19} \mathrm{~F}$ NMR $(471 \mathrm{MHz}$, Chloroform- $d$ ) $\delta$-73.49 ppm. ESI-HRMS m/z: $447.1737[\mathrm{M}+\mathrm{H}]{ }^{+}, \mathrm{C}_{20} \mathrm{H}_{25} \mathrm{ClF}_{3} \mathrm{~N}_{2} \mathrm{O}_{6}+\mathrm{H}^{+}$requires 447.1737.
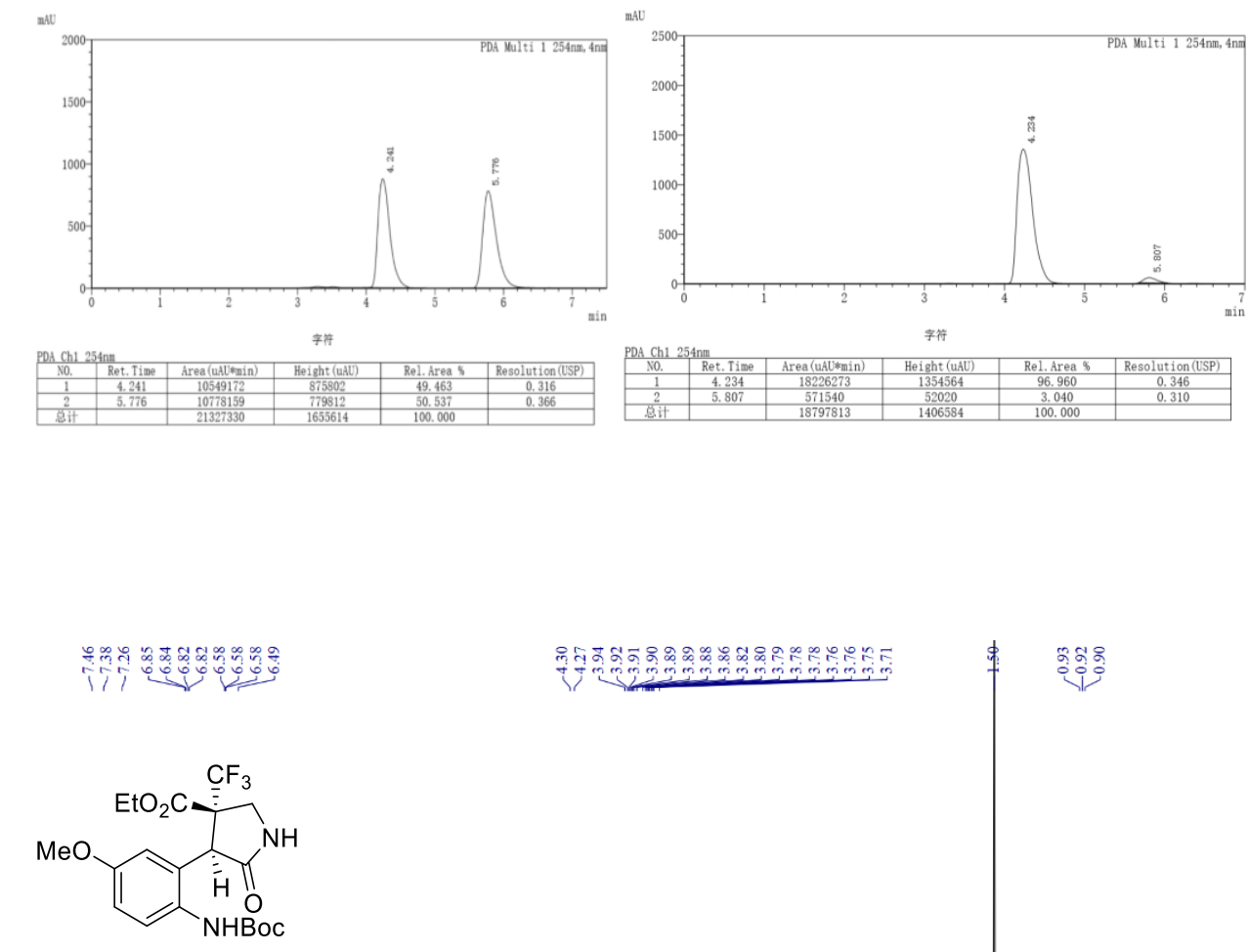

$3 c$

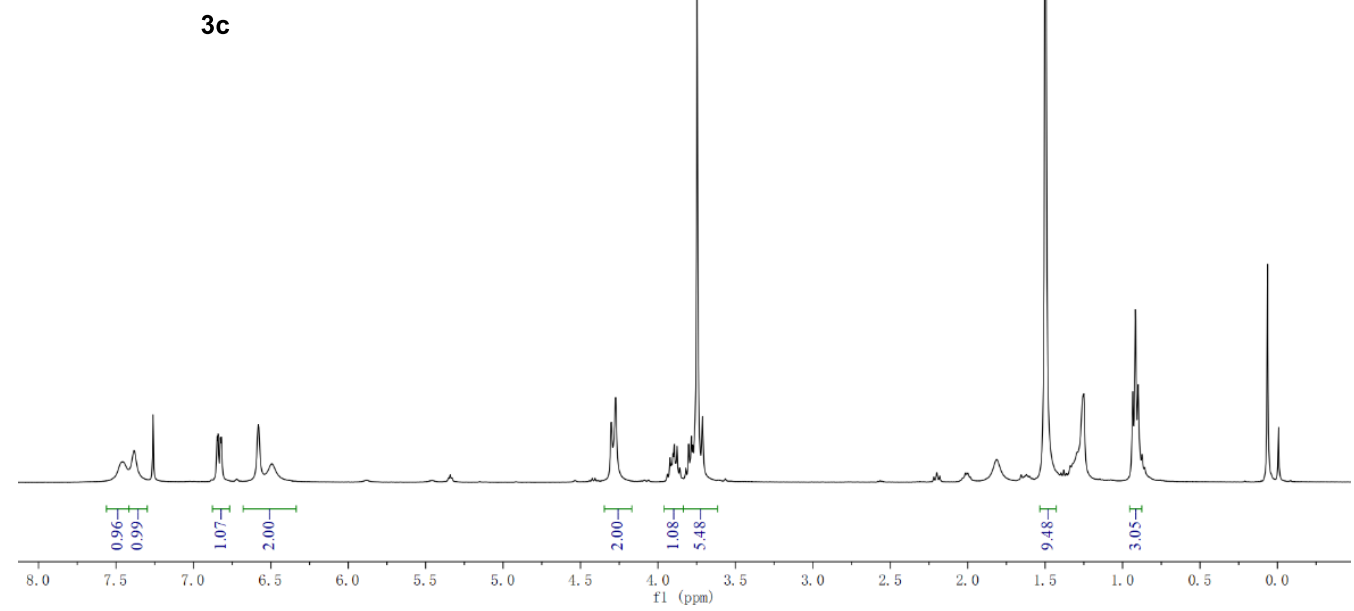



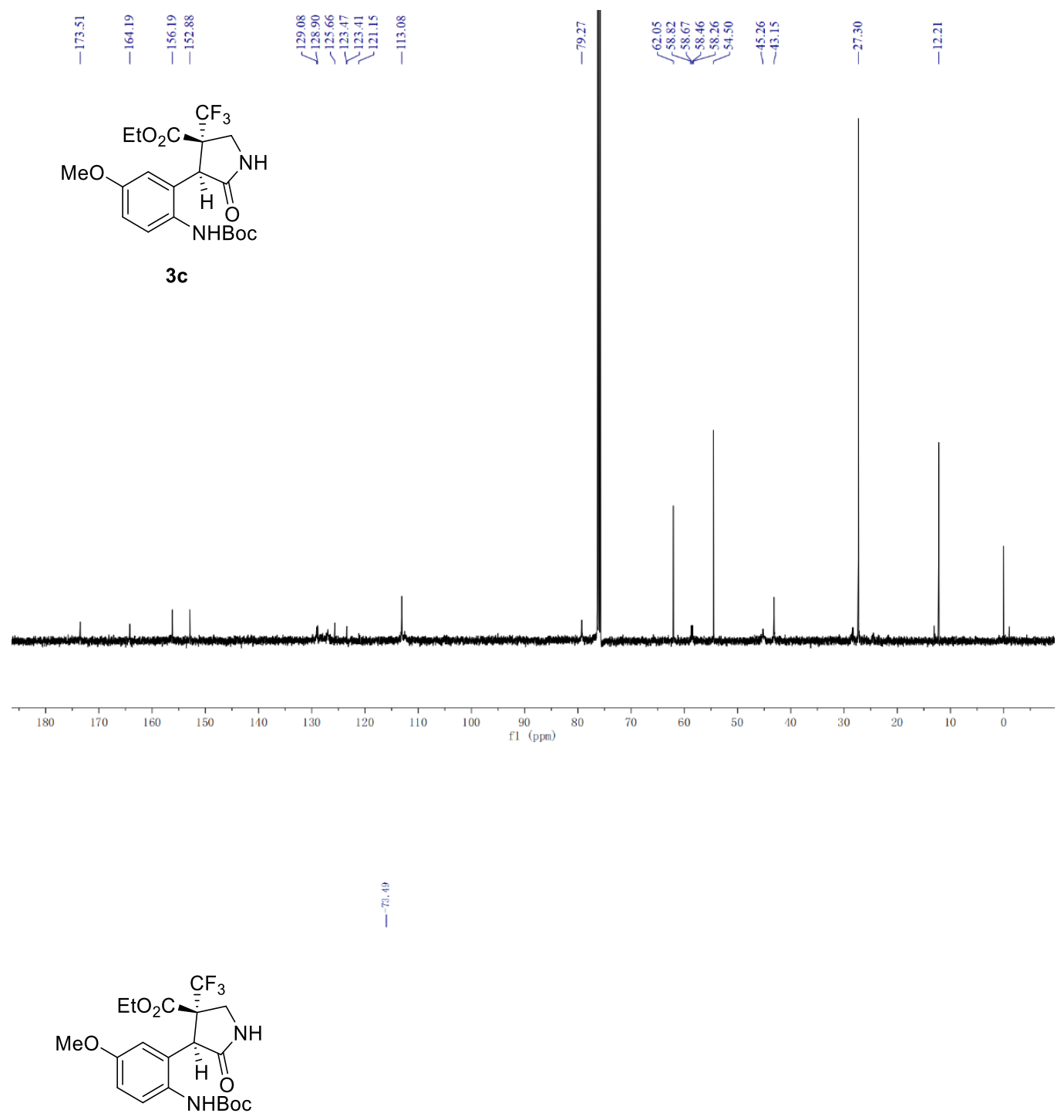

$3 c$
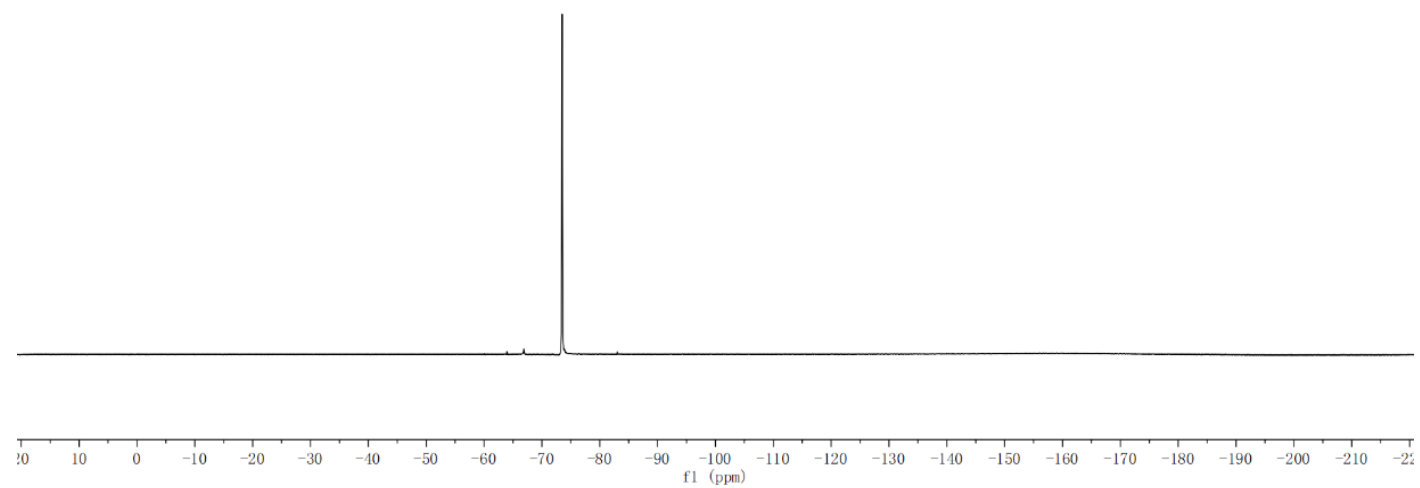

ethyl

(3S,4S)-4-(2-((tert-butoxycarbonyl)amino)-5-(trifluoromethyl)phenyl)-5-oxo-3-(trifluoromethyl) pyrrolidine-3-carboxylate (3d) 
<smiles>CCOC(=O)Nc1ccc(C(F)(F)F)cc1C1C(=O)NC[C@]1(C)C(F)(F)F</smiles>

$3 d$

3d was obtained as a yellow foam in $88 \%$ yield ( $3 \mathrm{~h}$ for Michael addition and $10 \mathrm{~h}$ for catalytic hydrogenation), 93\% ee and > 20:1 dr. The enantiomeric excess was determined by HPLC (Daicel Chiralpak AD-H, hexane $/ \mathrm{i}-\mathrm{PrOH}=90: 10(\mathrm{v} / \mathrm{v}), \lambda=254 \mathrm{~nm}$, flow rate $\left.=0.5 \mathrm{~mL} / \mathrm{min}, 25^{\circ} \mathrm{C}\right)$ : $t_{\text {minor }}=9.46 \mathrm{~min}, t_{\text {major }}=11.74 \mathrm{~min} ;[\alpha]_{\mathrm{D}}{ }^{25}-32.8(c 0.27, \mathrm{MeOH}) ;{ }^{1} \mathrm{H}$ NMR (500 MHz, Chloroform- $d$ ) $\delta 7.97$ (s, 1H), 7.71 (s, 1H), 7.33 (d, $J=$ $8.1 \mathrm{~Hz}, 1 \mathrm{H}), 7.15$ (d, J = 8.2 Hz, 1H), 6.99 (s, 1H), 4.36 (s, 1H), 4.30 (d, $J=12.1 \mathrm{~Hz}, 1 \mathrm{H}), 3.88(\mathrm{dq}, J=14.5,7.4 \mathrm{~Hz}, 1 \mathrm{H}), 3.75(\mathrm{~d}, J=12.0 \mathrm{~Hz}, 2 \mathrm{H}), 1.53(\mathrm{~s}, 9 \mathrm{H}), 0.89(\mathrm{t}, J$ $=7.1 \mathrm{~Hz}, 3 \mathrm{H}) \mathrm{ppm} .{ }^{13} \mathrm{C}$ NMR (125 MHz, Chloroform- $d$ ) $\delta$ 173.70, 164.93, 152.82, 138.27, 131.49 $(\mathrm{q}, J=32.7 \mathrm{~Hz}), 129.67,125.41(\mathrm{q}, J=283.0 \mathrm{~Hz}), 123.48(\mathrm{q}, J=272.5 \mathrm{~Hz}), 121.93,121.29,81.30$, 63.36, $59.50(\mathrm{q}, J=26.8,26.3 \mathrm{~Hz}), 46.00,44.11,28.22,13.12 \mathrm{ppm} ;{ }^{19} \mathrm{~F}$ NMR $(471 \mathrm{MHz}$, Chloroform- $d$ ) $\delta$-63.02, -73.34 ppm. ESI-HRMS m/z: $485.1504[\mathrm{M}+\mathrm{H}]{ }^{+}, \mathrm{C}_{20} \mathrm{H}_{22} \mathrm{~F}_{6} \mathrm{~N}_{2} \mathrm{O}_{5}+\mathrm{H}^{+}$ requires 485.1506 .
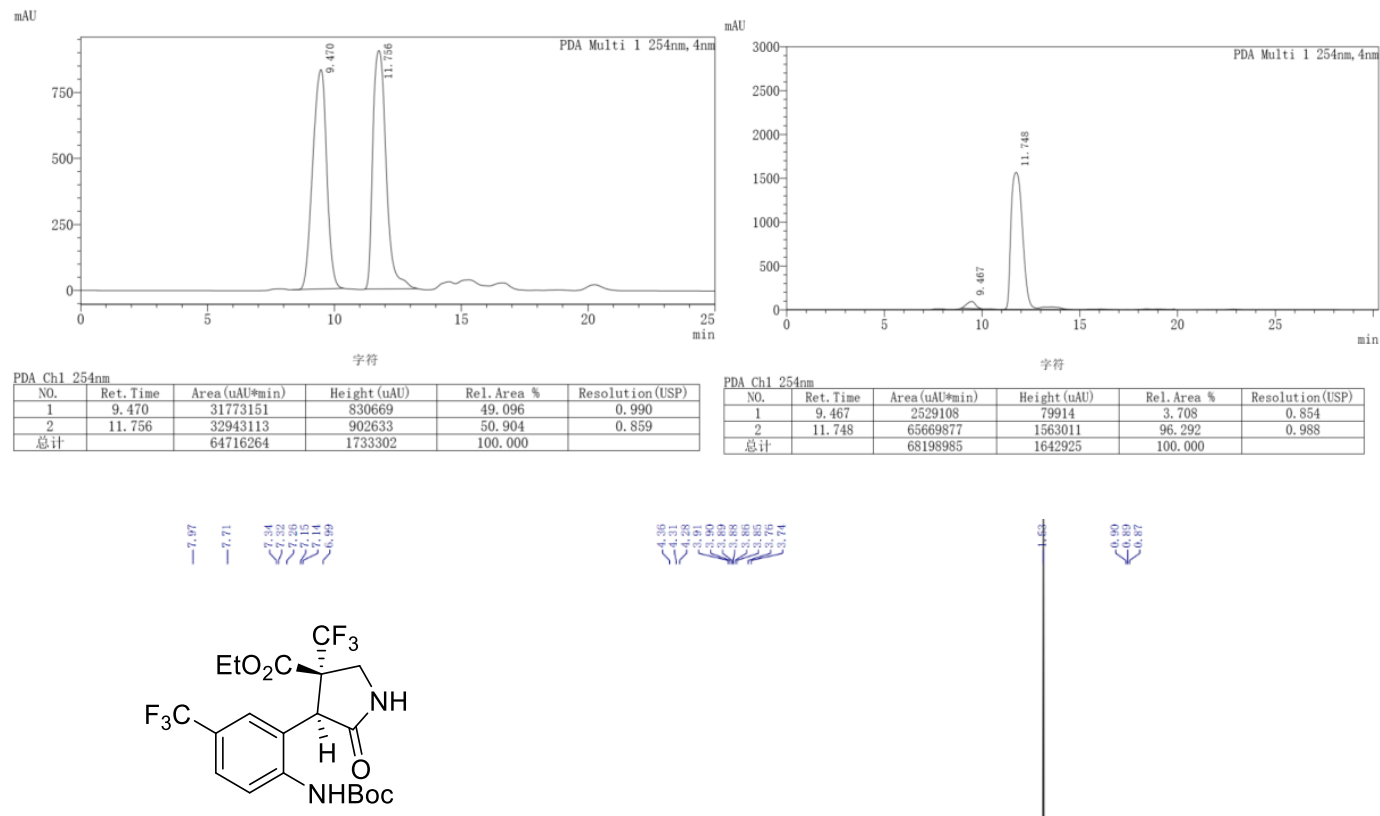

$3 d$
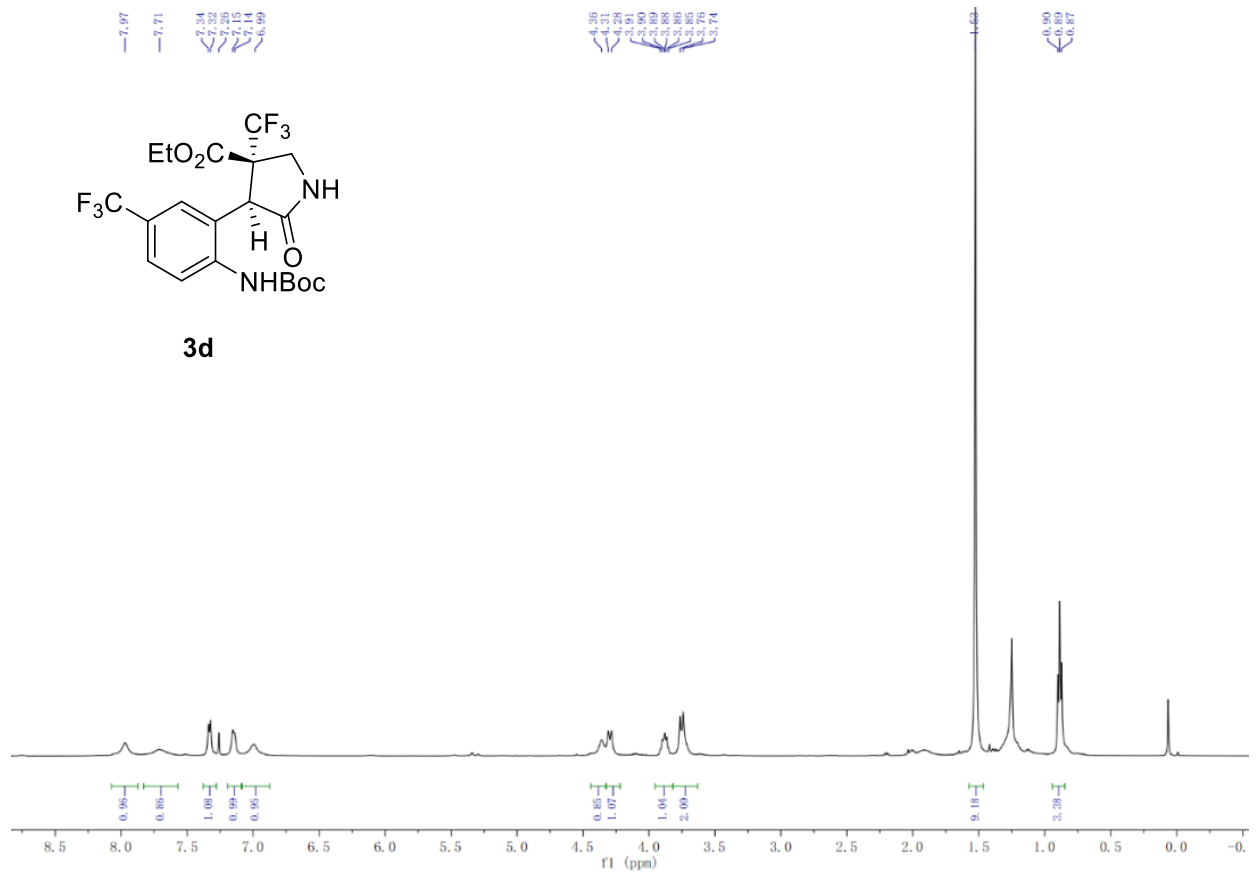

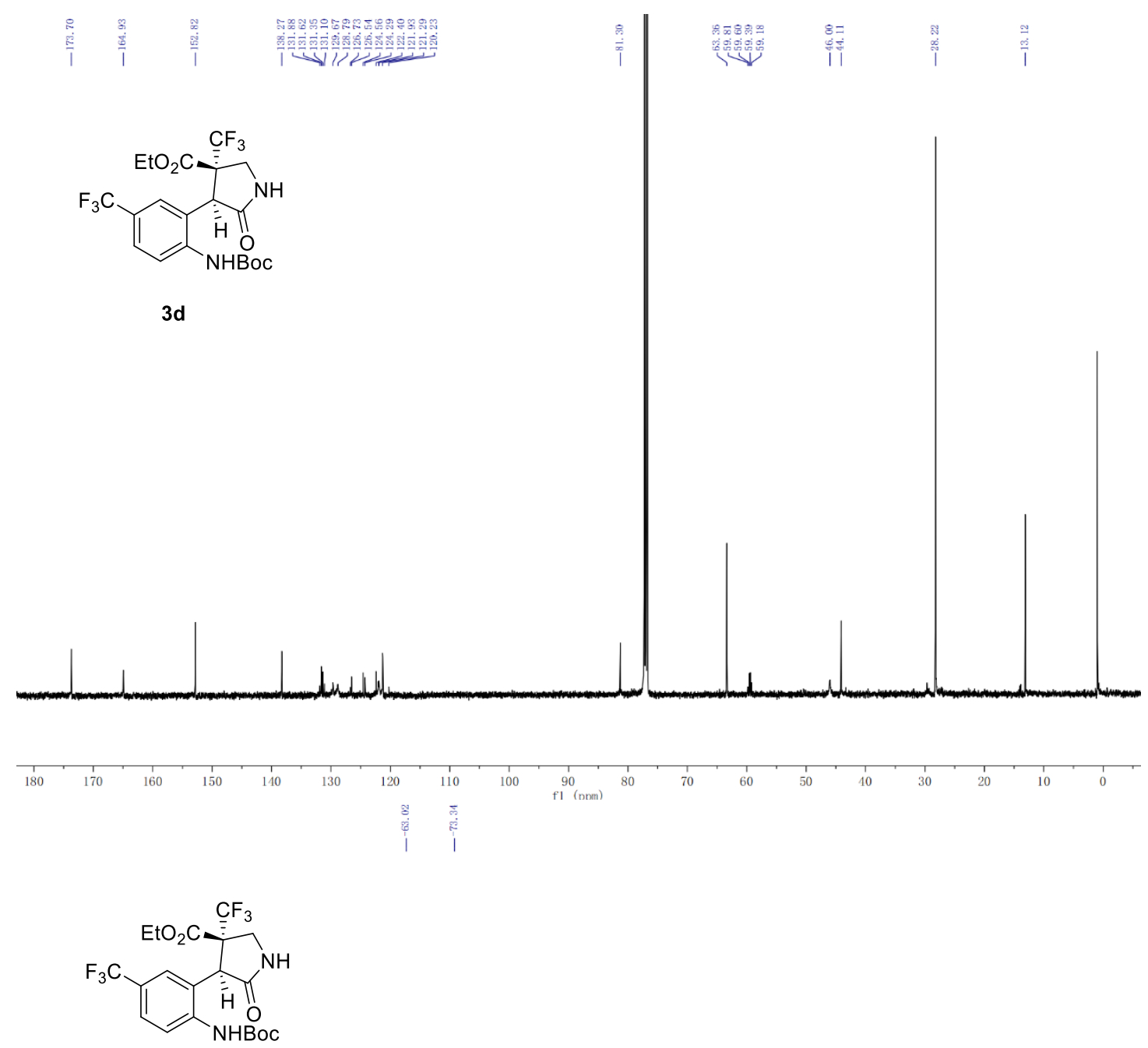

3d

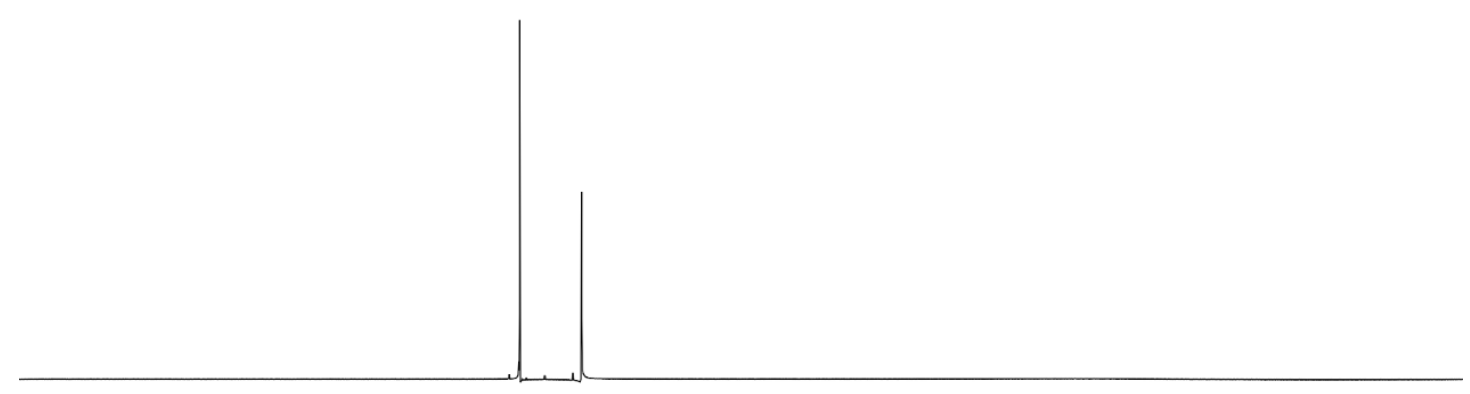

$\begin{array}{llllllllllllllllllllllllllll}1 & 1 \\ 30 & 10 & 0 & -10 & -20 & -30 & -40 & -50 & -60 & -70 & -80 & -90 & -100 & -110 & -120 & -130 & -140 & -150 & -160 & -170 & -180 & -190 & -200 & -210 & -22\end{array}$ 
ethyl (3S,4S)-4-(2-((tert-butoxycarbonyl)amino)-5-fluorophenyl)-5-oxo-3-(trifluoromethyl)pyrrolidine-3-carboxylate (3e)<smiles>CCOC(=O)Nc1ccc(F)cc1C1C(=O)NCC1(C(=O)OCC)C(F)(F)F</smiles>

$3 e$

3e was obtained as a yellow foam in $88 \%$ yield ( $4 \mathrm{~h}$ for Michael addition and $12 \mathrm{~h}$ for catalytic hydrogenation), $93 \%$ ee and > 20:1 dr. The enantiomeric excess was determined by HPLC (Daicel Chiralpak AD-H, hexane/i-PrOH $=90: 10(\mathrm{v} / \mathrm{v}), \lambda=254 \mathrm{~nm}$, flow rate $\left.=0.5 \mathrm{~mL} / \mathrm{min}, 25^{\circ} \mathrm{C}\right)$ : $t_{\text {minor }}=12.41 \mathrm{~min}, t_{\text {major }}=16.83 \mathrm{~min} ;[\alpha]_{\mathrm{D}}{ }^{25} 23.2(c 0.17, \mathrm{MeOH}) ;{ }^{1} \mathrm{H} \mathrm{NMR}$ $(500 \mathrm{MHz}$, Chloroform- $d$ ) $\delta 7.56(\mathrm{~s}, 1 \mathrm{H}), 7.44(\mathrm{~s}, 1 \mathrm{H}), 7.01$ (ddd, $J=8.9$, 7.7, $2.9 \mathrm{~Hz}, 1 \mathrm{H}), 6.79(\mathrm{~d}, J=9.0 \mathrm{~Hz}, 1 \mathrm{H}), 6.67(\mathrm{~s}, 1 \mathrm{H}), 4.27$ (d, $J=12.1 \mathrm{~Hz}$ 2H), $3.92(\mathrm{dq}, J=10.7,7.1 \mathrm{~Hz}, 1 \mathrm{H}), 3.80(\mathrm{dd}, J=10.8,7.1 \mathrm{~Hz}, 1 \mathrm{H}), 3.74(\mathrm{dd}, J=11.8,1.4 \mathrm{~Hz}$, 1H), $1.50(\mathrm{~s}, 9 \mathrm{H}), 0.94(\mathrm{t}, J=7.1 \mathrm{~Hz}, 3 \mathrm{H}) \mathrm{ppm} .{ }^{13} \mathrm{C}$ NMR $(125 \mathrm{MHz}$, Chloroform- $d$ ) $\delta 174.27$, 165.07, 160.73, 158.78, 153.60, 133.46, 127.95, 125.51 (q, $J=282.6 \mathrm{~Hz}), 115.94$ (d, $J=22.0 \mathrm{~Hz})$, 114.85, 80.60, 63.17, 59.47 (q, $J=26.0 \mathrm{~Hz}), 46.36,44.21,28.22,13.17 \mathrm{ppm} ;{ }^{19} \mathrm{~F}$ NMR $(471 \mathrm{MHz}$, Chloroform- $d$ ) $\delta$-73.44, -116.07 ppm. ESI-HRMS m/z: $435.1535[\mathrm{M}+\mathrm{H}]{ }^{+}, \mathrm{C}_{19} \mathrm{H}_{22} \mathrm{~F}_{4} \mathrm{~N}_{2} \mathrm{O}_{5}+\mathrm{H}^{+}$ requires 435.1538 .
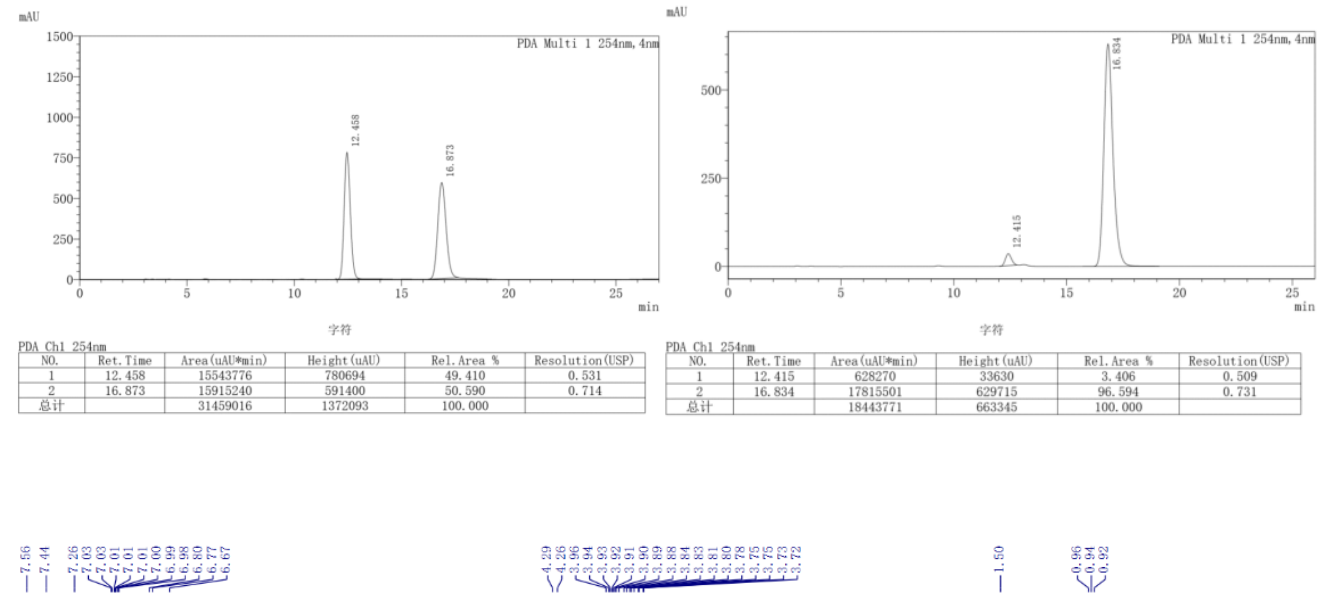<smiles>CCOC(=O)C1(C(F)(F)F)CNC(=O)C1c1cc(F)ccc1NC(=O)OCc1ccccc1</smiles>

$3 e$

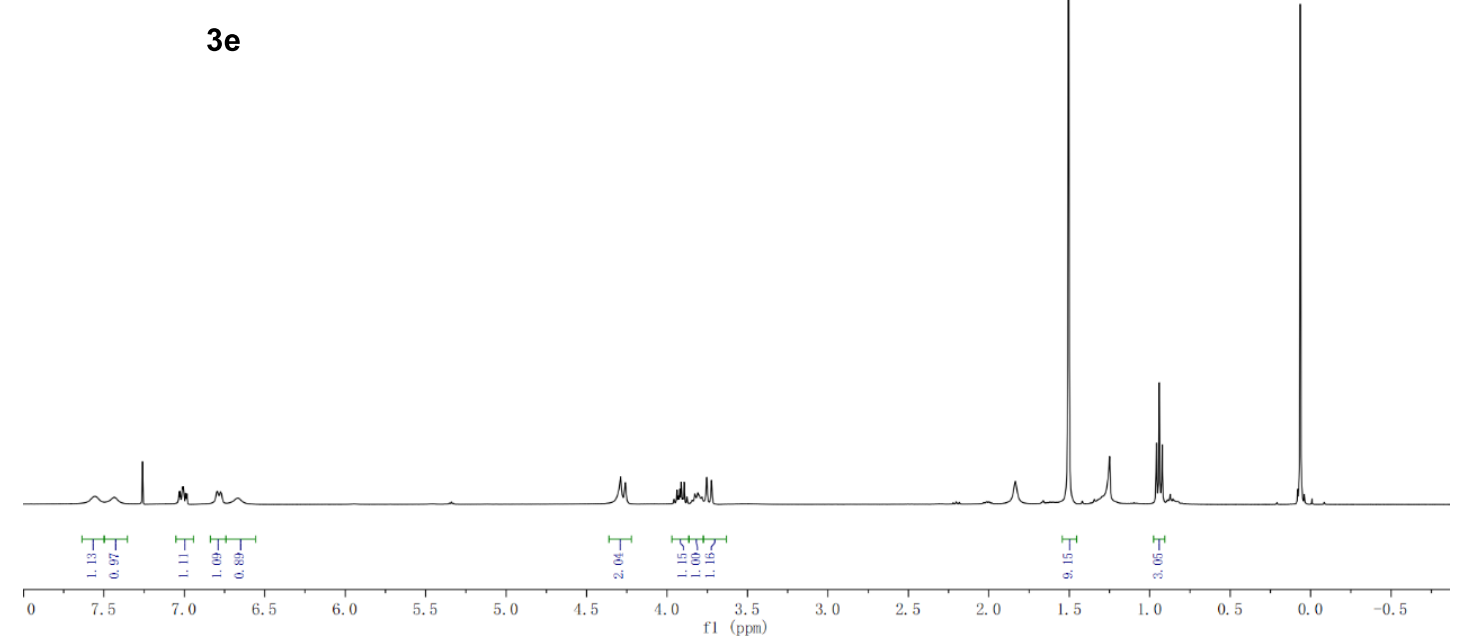



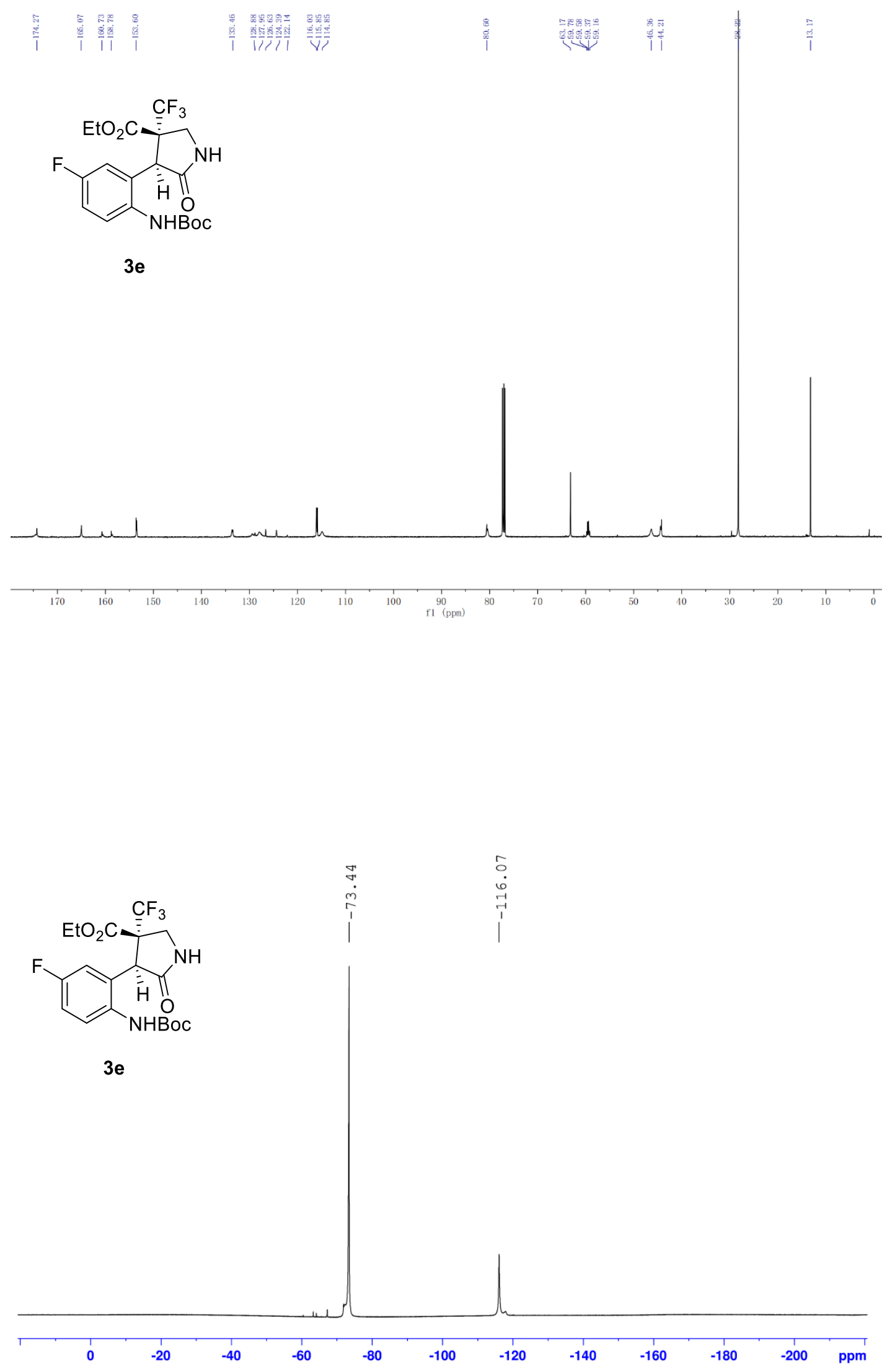
(3S,4S)-4-(2-((tert-butoxycarbonyl)amino)-5-chlorophenyl)-5-oxo-3-(trifluoromethyl)pyrrolidine-3-carboxylate (3f)<smiles>CCOC(=O)Nc1ccc(Cl)cc1C1C(=O)NCC1C(F)(F)F</smiles>

$3 \mathbf{f}$

3f was obtained as a light yellow foam in $89 \%$ yield ( $4 \mathrm{~h}$ for Michael addition and $10 \mathrm{~h}$ for catalytic hydrogenation), $90 \%$ ee and $>20: 1 \mathrm{dr}$. The enantiomeric excess was determined by HPLC (Daicel Chiralpak AD-H, hexane $/ \mathrm{i}-\mathrm{PrOH}=80: 20(\mathrm{v} / \mathrm{v}), \lambda=254 \mathrm{~nm}$, flow rate $\left.=1.0 \mathrm{~mL} / \mathrm{min}, 25^{\circ} \mathrm{C}\right)$ : $t_{\text {minor }}=4.95 \mathrm{~min}, t_{\text {major }}=3.69 \mathrm{~min} ;[\alpha]_{\mathrm{D}}{ }^{25} 13.4($ c $0.26, \mathrm{MeOH}) ;{ }^{1} \mathrm{H} \mathrm{NMR}$ $(500 \mathrm{MHz}$, Chloroform- $d$ ) $\delta 7.74(\mathrm{~s}, 1 \mathrm{H}), 7.64-7.49(\mathrm{~m}, 1 \mathrm{H}), 7.30-7.14$ $(\mathrm{m}, 1 \mathrm{H}), 7.01(\mathrm{~s}, 1 \mathrm{H}), 6.78(\mathrm{~s}, 1 \mathrm{H}), 4.41-4.15(\mathrm{~m}, 2 \mathrm{H}), 3.90(\mathrm{dt}, J=14.0,7.1 \mathrm{~Hz}, 1 \mathrm{H}), 3.86-$ $3.77(\mathrm{~m}, 1 \mathrm{H}), 3.72(\mathrm{~d}, J=11.7 \mathrm{~Hz}, 1 \mathrm{H}), 1.50(\mathrm{~s}, 9 \mathrm{H}), 0.93(\mathrm{td}, J=7.1,2.7 \mathrm{~Hz}, 3 \mathrm{H}) \mathrm{ppm} .{ }^{13} \mathrm{C}$ NMR (125 MHz, Chloroform- $d$ ) $\delta 174.11,165.00,153.19,136.14,130.36,129.17,128.25,126.81$, 125.45 (q, $J=282.9 \mathrm{~Hz}), 124.38,80.84,63.24,59.48$ (q, $J=25.2 \mathrm{~Hz}), 46.16,44.19,28.23,13.21$ ppm; ${ }^{19} \mathrm{~F}$ NMR (471 MHz, Chloroform- $d$ ) $\delta-73.39$ ppm. ESI-HRMS m/z: $451.1239[\mathrm{M}+\mathrm{H}]{ }^{+}$, $\mathrm{C}_{19} \mathrm{H}_{22} \mathrm{ClF}_{3} \mathrm{~N}_{2} \mathrm{O}_{5}+\mathrm{H}^{+}$requires 451.1242 .
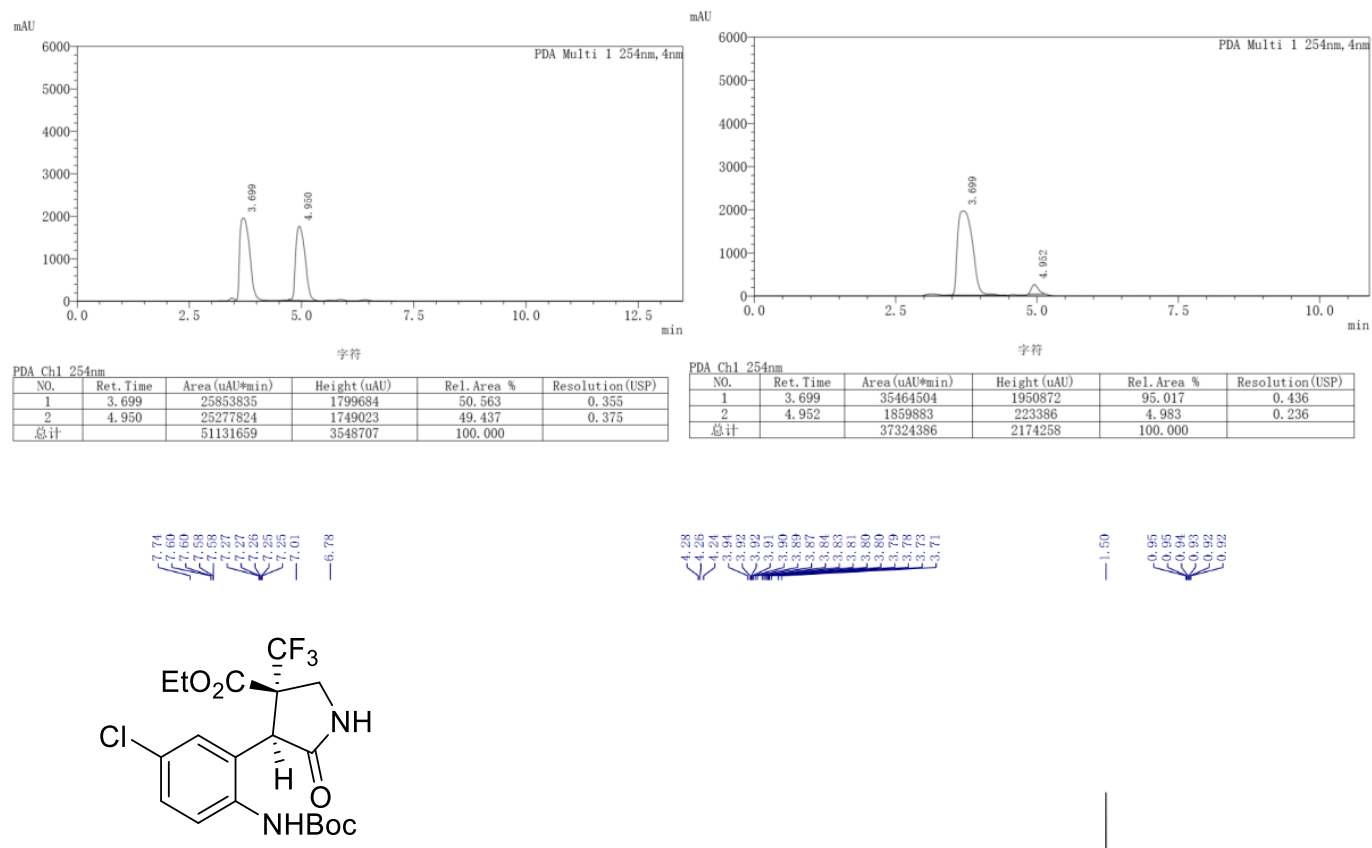

$3 f$

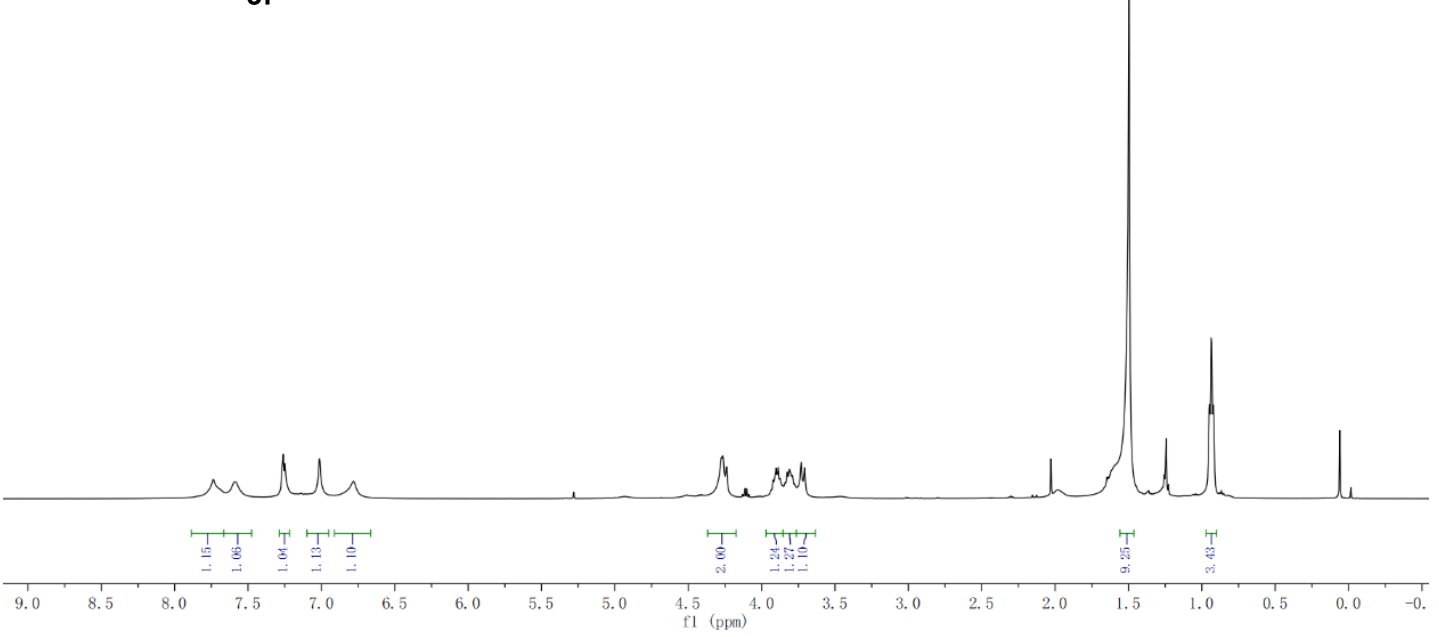




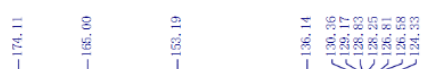

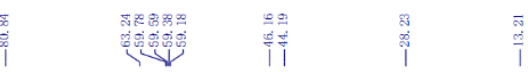

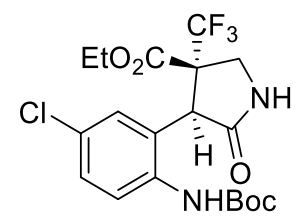

$3 f$
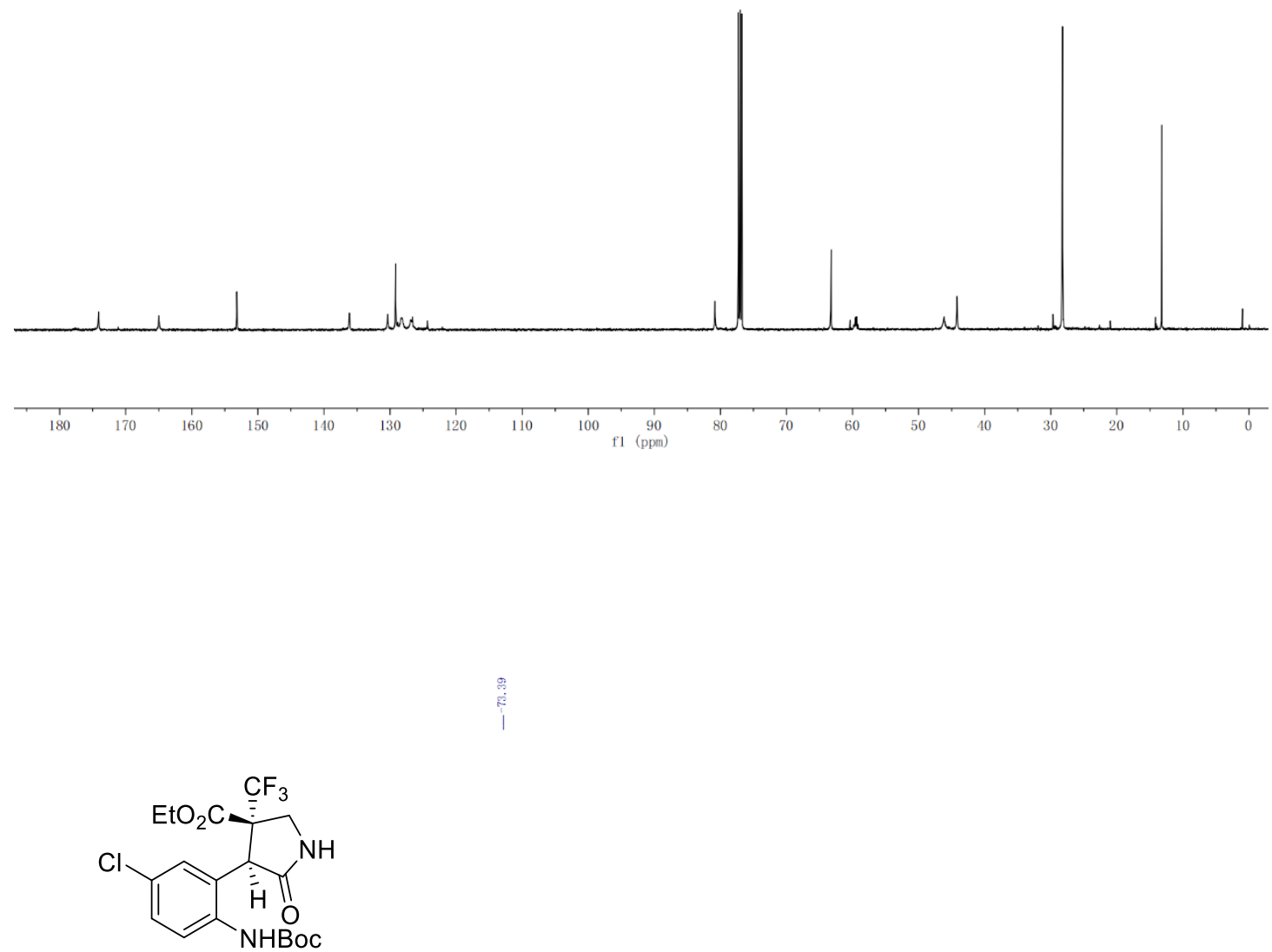

$3 f$

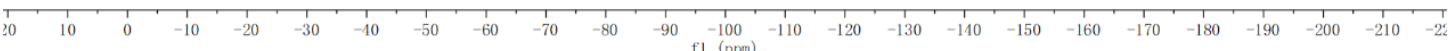


(3S,4S)-4-(5-bromo-2-((tert-butoxycarbonyl)amino)phenyl)-5-oxo-3-(trifluoromethyl)pyrrolidine-3-carboxylate (3g)<smiles>CCOC(=O)c1cc(Br)ccc1NC(=O)OC(C)(F)C1C(=O)NCC1C(F)(F)F</smiles>

$3 \mathbf{g}$

$3 \mathrm{~g}$ was obtained as a yellow foam in $90 \%$ yield ( $4 \mathrm{~h}$ for Michael addition and $11 \mathrm{~h}$ for catalytic hydrogenation), 93\% ee and > 20:1 dr. The enantiomeric excess was determined by HPLC (Daicel Chiralpak AD-H, hexane/i-PrOH $=80: 20(\mathrm{v} / \mathrm{v}), \lambda=254 \mathrm{~nm}$, flow rate $\left.=1.0 \mathrm{~mL} / \mathrm{min}, 25{ }^{\circ} \mathrm{C}\right)$ : $t_{\text {minor }}=4.97 \mathrm{~min}, t_{\text {major }}=3.82 \mathrm{~min} ;[\alpha]_{\mathrm{D}}{ }^{25} 17.2(c 0.37, \mathrm{MeOH}) ;{ }^{1} \mathrm{H} \mathrm{NMR}$ $(500 \mathrm{MHz}$, Chloroform- $d) \delta 7.66(\mathrm{~s}, 1 \mathrm{H}), 7.30(\mathrm{td}, J=8.3,7.8,1.7 \mathrm{~Hz}, 1 \mathrm{H})$, $7.11(\mathrm{td}, J=7.6,1.3 \mathrm{~Hz}, 1 \mathrm{H}), 7.05(\mathrm{~d}, J=7.8 \mathrm{~Hz}, 1 \mathrm{H}), 6.71(\mathrm{~s}, 1 \mathrm{H}), 4.30(\mathrm{~m}, 2 \mathrm{H}), 3.87(\mathrm{dq}, J=$ 10.7, 7.1 Hz, 1H), $3.81-3.57(\mathrm{~m}, 2 \mathrm{H}), 1.52(\mathrm{~s}, 9 \mathrm{H}), 0.90(\mathrm{t}, J=7.1 \mathrm{~Hz}, 3 \mathrm{H}) \mathrm{ppm} .{ }^{13} \mathrm{C}$ NMR $(125$ MHz, Chloroform- $d$ ) $\delta 174.48,165.16,153.31,137.31,129.12,126.80,125.60,125.60$ (q, $J=$ $283.1 \mathrm{~Hz}$ ), 125.23, 125.19, 80.53, 63.09, 59.64 (q, $J=24.5 \mathrm{~Hz}), 44.07,29.68,28.29,13.18$ ppm; ${ }^{19} \mathrm{~F}$ NMR (471 MHz, Chloroform- $d$ ) $\delta-73.41 \mathrm{ppm}$. ESI-HRMS m/z: $495.0738[\mathrm{M}+\mathrm{H}]{ }^{+}$, $\mathrm{C}_{19} \mathrm{H}_{22} \mathrm{BrF}_{3} \mathrm{~N}_{2} \mathrm{O}_{5}+\mathrm{H}^{+}$requires 495.0736 .
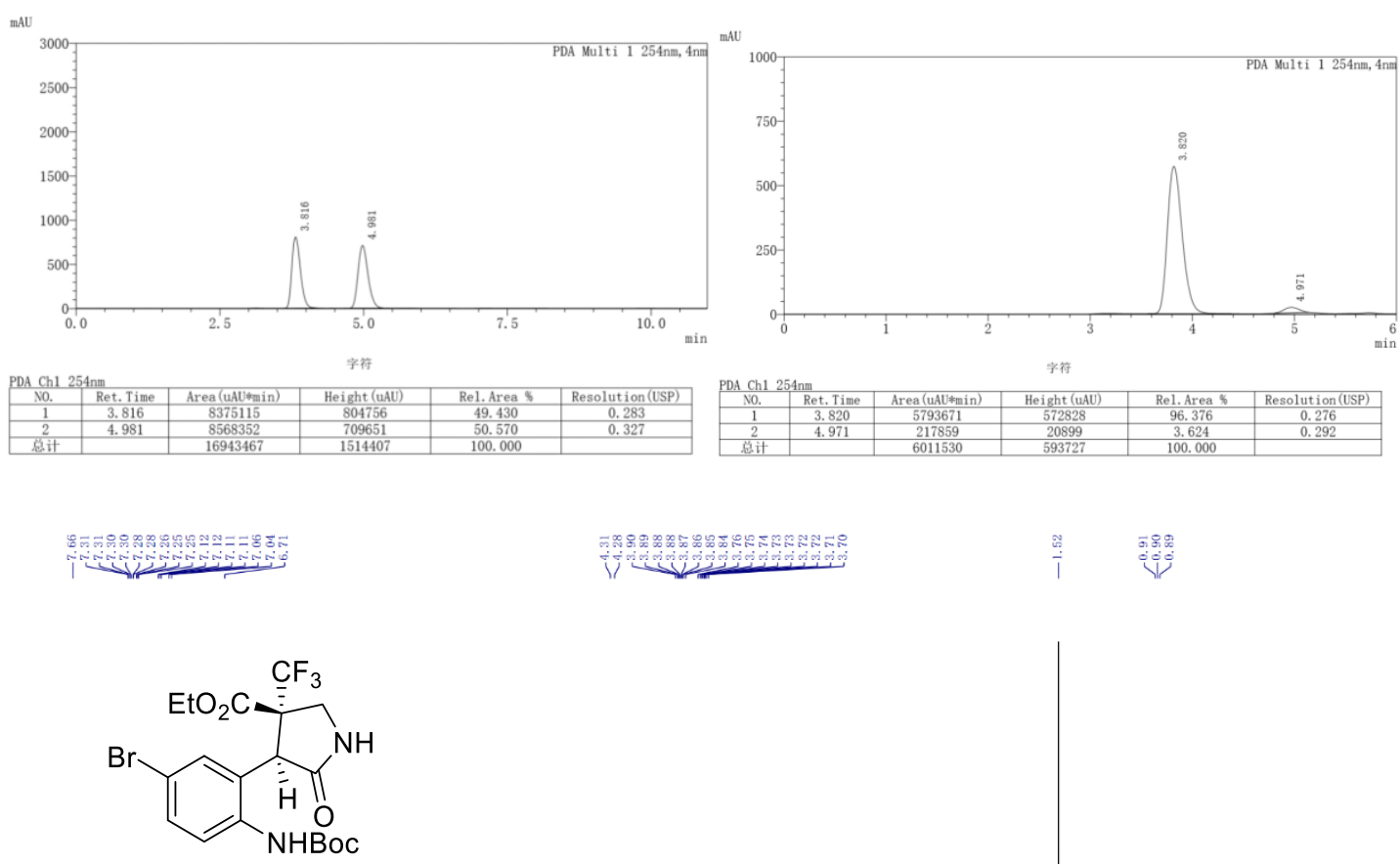

$3 \mathbf{g}$

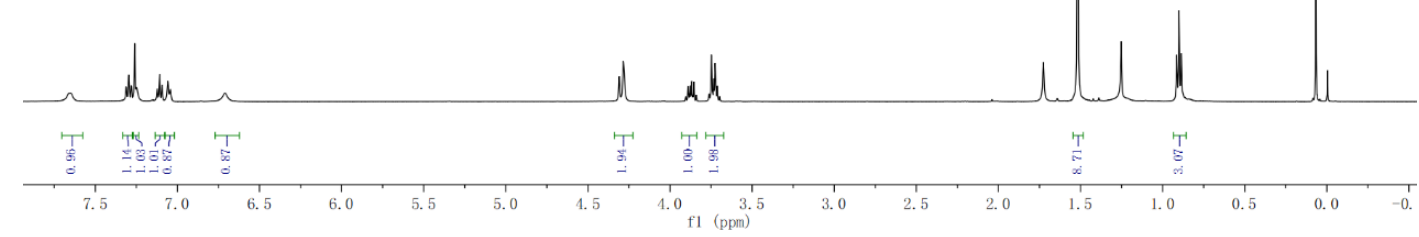



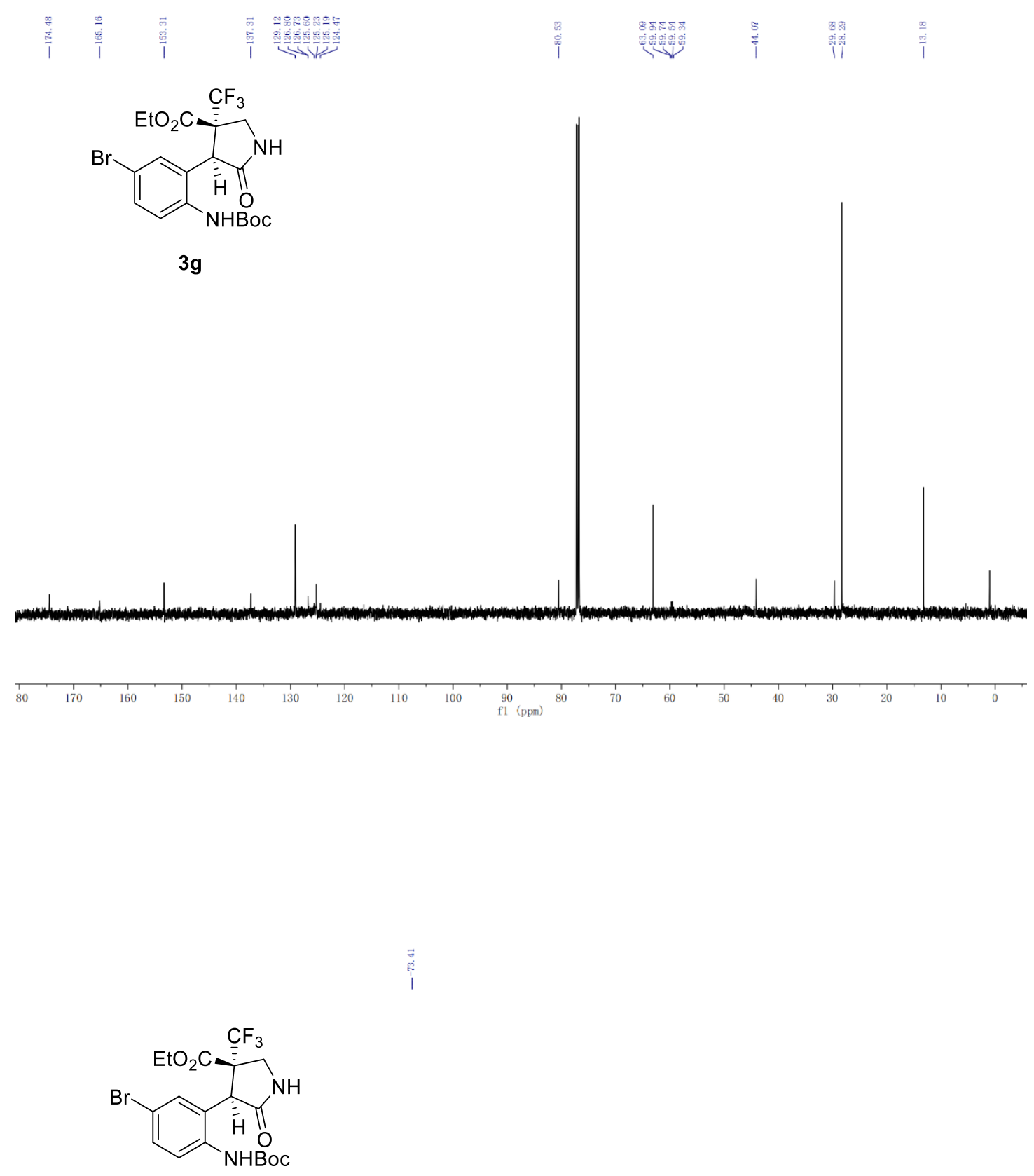

$3 g$

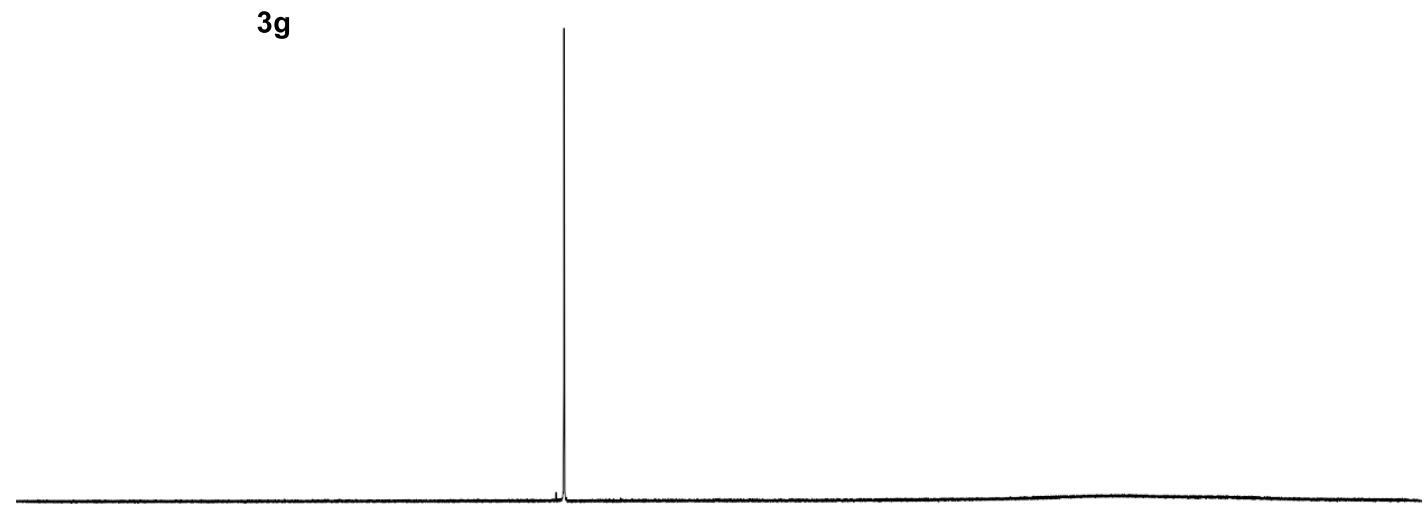

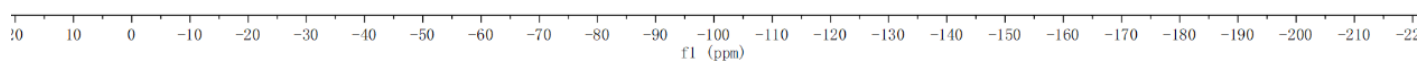


ethyl (3S,4S)-4-(2-((tert-butoxycarbonyl) amino)-4-chlorophenyl)-5-oxo-3-(trifluoromethyl)pyrrolidine-3-carboxylate (3h)

3h was obtained as a yellow foam in $91 \%$ yield ( $4 \mathrm{~h}$ for Michael addition<smiles>CCOC(=O)c1ccc(Cl)cc1NC(=O)OC(C)(C)C1(C(F)(F)F)CNC(=O)C1c1ccccc1</smiles>

$3 h$ and $10 \mathrm{~h}$ for catalytic hydrogenation), $93 \%$ ee and > 20:1 dr. The enantiomeric excess was determined by HPLC (Daicel Chiralpak OD-H, hexane/i-PrOH $=90: 10(\mathrm{v} / \mathrm{v}), \lambda=254 \mathrm{~nm}$, flow rate $\left.=0.5 \mathrm{~mL} / \mathrm{min}, 25{ }^{\circ} \mathrm{C}\right)$ : $t_{\text {minor }}=11.95 \mathrm{~min}, t_{\text {major }}=9.83 \mathrm{~min} ;[\alpha]_{\mathrm{D}}{ }^{25} 8.24(c 0.36, \mathrm{MeOH}) ;{ }^{1} \mathrm{H} \mathrm{NMR}$ (400 MHz, DMSO- $\left.d_{6}\right) \delta 9.05(\mathrm{~s}, 1 \mathrm{H}), 8.54(\mathrm{~s}, 1 \mathrm{H}), 7.49(\mathrm{~d}, J=2.2 \mathrm{~Hz}, 1 \mathrm{H})$, $7.17(\mathrm{dd}, J=8.5,2.3 \mathrm{~Hz}, 1 \mathrm{H}), 6.92(\mathrm{~d}, J=8.4 \mathrm{~Hz}, 1 \mathrm{H}), 4.79(\mathrm{~s}, 1 \mathrm{H}), 4.02(\mathrm{~d}$, $J=11.6 \mathrm{~Hz}, 1 \mathrm{H}), 3.81(\mathrm{dq}, J=10.6,7.1 \mathrm{~Hz}, 1 \mathrm{H}), 3.70(\mathrm{dq}, J=10.8,7.1 \mathrm{~Hz}, 1 \mathrm{H}), 3.61(\mathrm{~d}, J=11.6$ $\mathrm{Hz}, 1 \mathrm{H}), 1.46(\mathrm{~s}, 9 \mathrm{H}), 0.87$ (t, $J=7.1 \mathrm{~Hz}, 3 \mathrm{H}) \mathrm{ppm} .{ }^{13} \mathrm{C}$ NMR $\left(125 \mathrm{MHz}\right.$, DMSO- $\left.d_{6}\right) \delta 173.01$, 165.65, 153.83, 140.09, 132.73, 131.19, 126.81, 125.95 (q, $J=282.2 \mathrm{~Hz}), 125.01,124.35,79.59$, $62.71,59.47(\mathrm{q}, J=25.7 \mathrm{~Hz}), 44.93,43.94,28.49,13.42 \mathrm{ppm} ;{ }^{19} \mathrm{~F}$ NMR (471 MHz, DMSO- $\left.d_{6}\right) \delta$ -71.26 ppm. ESI-HRMS m/z: $451.1237[\mathrm{M}+\mathrm{H}]{ }^{+}, \mathrm{C}_{19} \mathrm{H}_{22} \mathrm{ClF}_{3} \mathrm{~N}_{2} \mathrm{O}_{5}+\mathrm{H}^{+}$requires 451.1242.
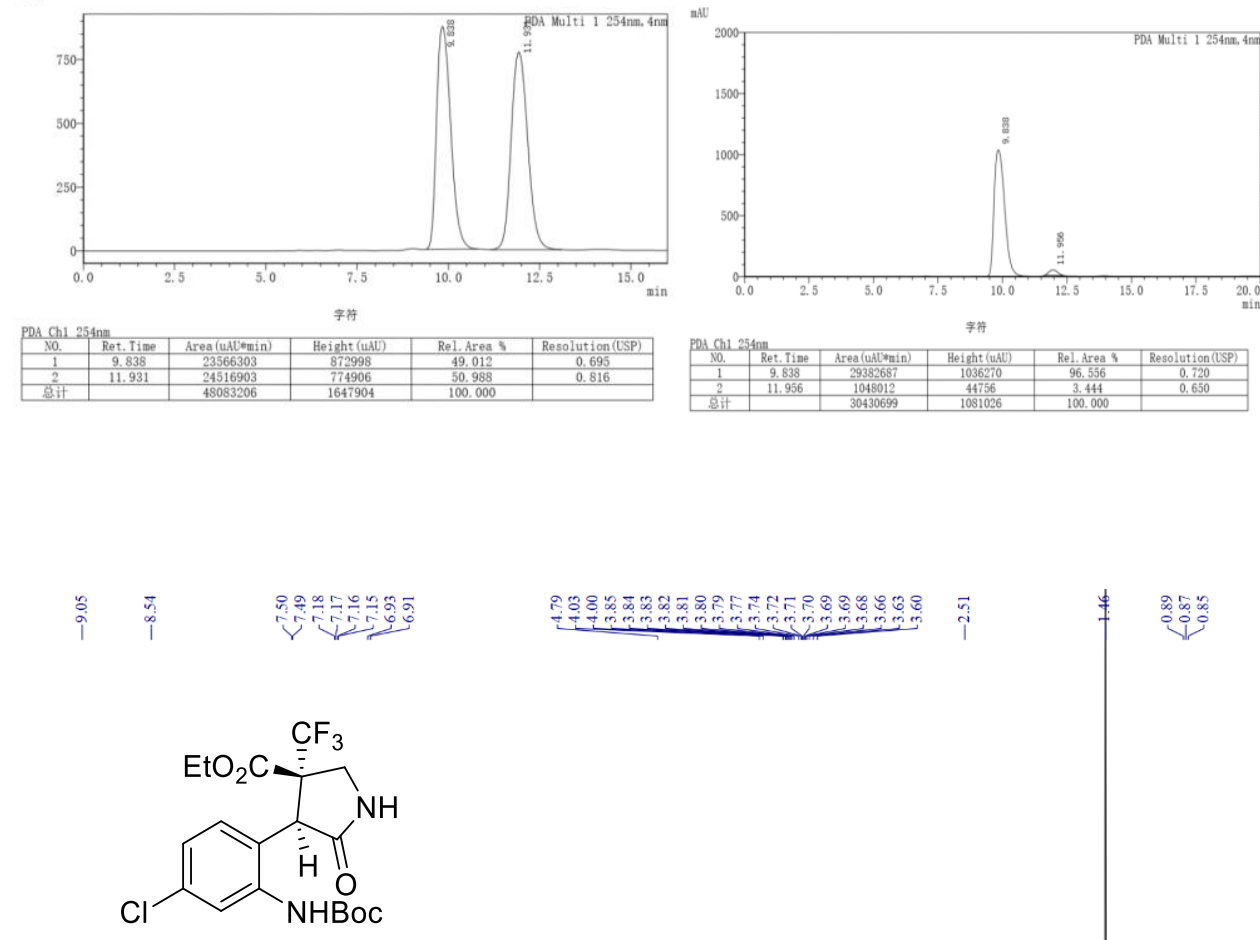

$3 h$
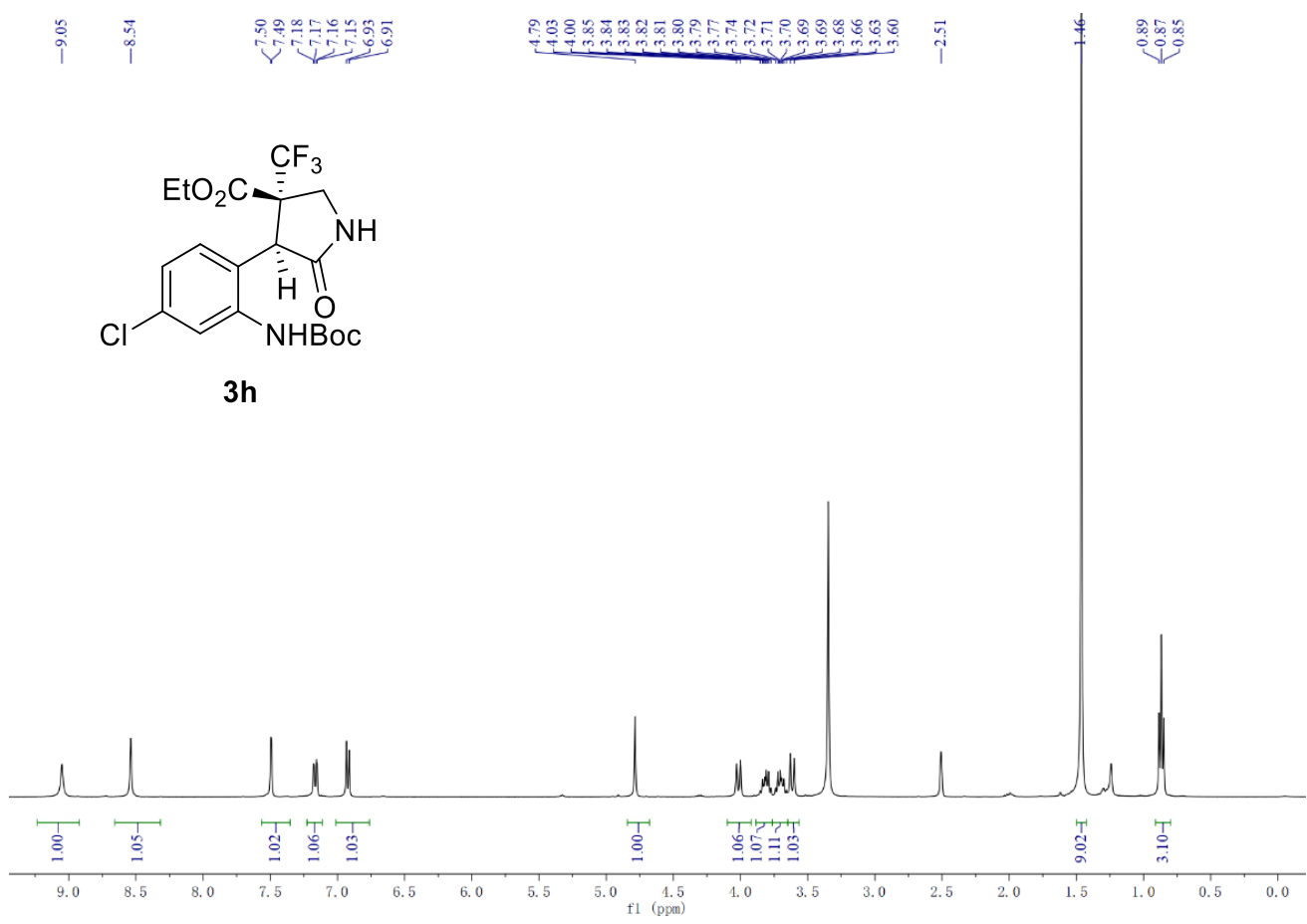

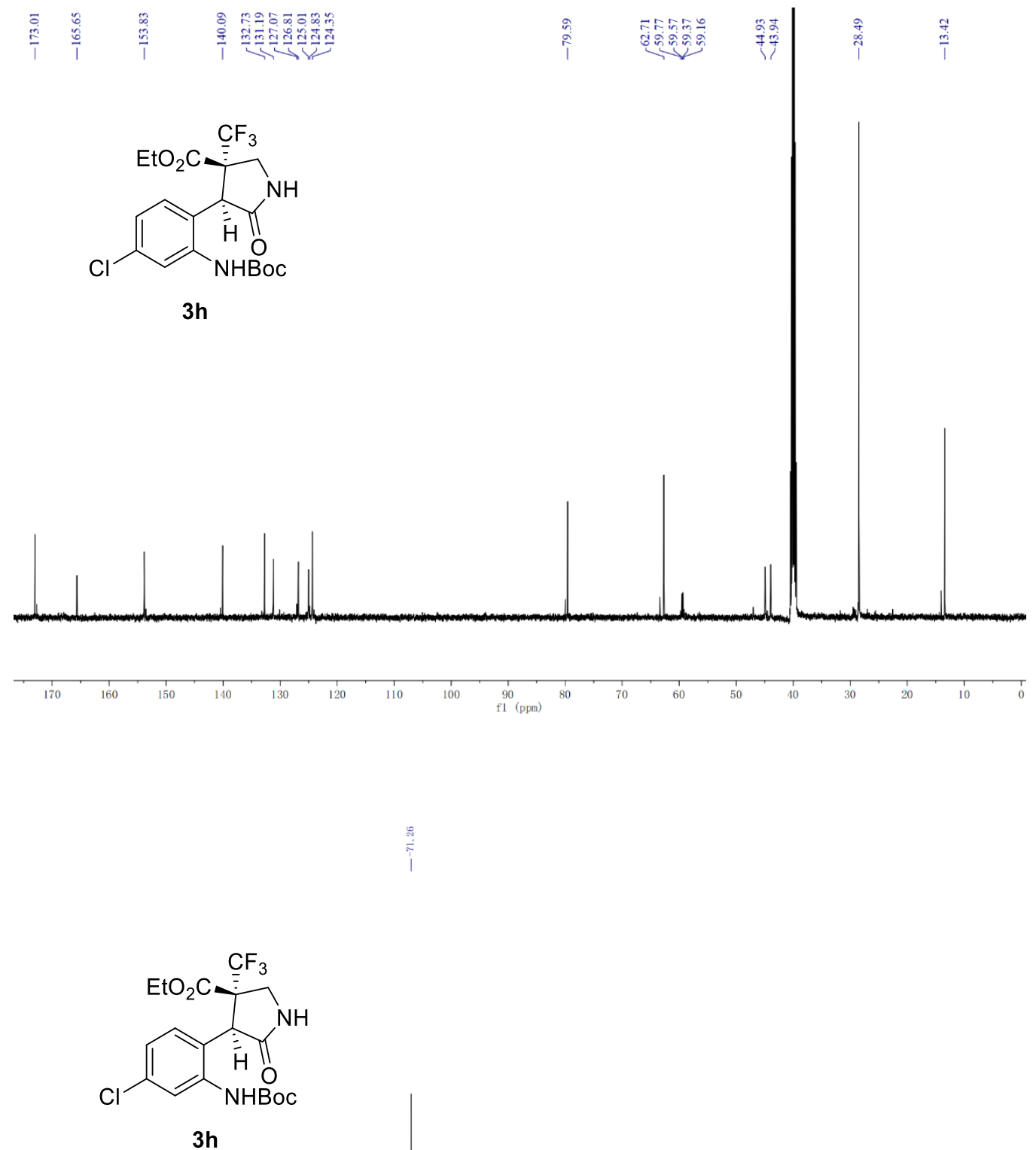

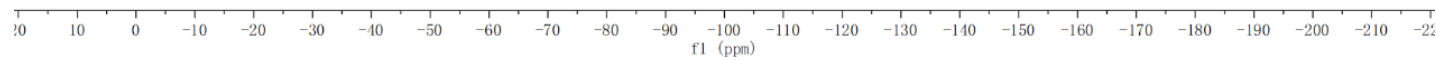


ethyl (3S,4S)-4-(4-bromo-2-((tert-butoxycarbonyl) amino) phenyl)-5-oxo-3-(trifluoromethyl)pyrrolidine-3-carboxylate (3i)<smiles>CCOC(=O)c1ccc(Br)cc1NC(=O)OC1C(=O)NCC1(C(F)(F)F)C(F)(F)F</smiles>

$3 \mathbf{i}$

3i was obtained as a yellow foam in $93 \%$ yield ( $3 \mathrm{~h}$ for Michael addition and $10 \mathrm{~h}$ for catalytic hydrogenation), $96 \%$ ee and > 20:1 dr. The enantiomeric excess was determined by HPLC (Daicel Chiralpak OD-H, hexane $/ \mathrm{i}-\mathrm{PrOH}=90: 10(\mathrm{v} / \mathrm{v}), \lambda=254 \mathrm{~nm}$, flow rate $\left.=0.5 \mathrm{~mL} / \mathrm{min}, 25{ }^{\circ} \mathrm{C}\right)$ : $t_{\text {minor }}=12.30 \mathrm{~min}, t_{\text {major }}=10.03 \mathrm{~min} ;[\alpha]_{\mathrm{D}}{ }^{25} 11.4(c 0.30, \mathrm{MeOH}) ;{ }^{1} \mathrm{H}$ NMR (400 MHz, Chloroform- $d$ ) $\delta 7.93(\mathrm{~s}, 1 \mathrm{H}), 7.23(\mathrm{~d}, J=8.3 \mathrm{~Hz}, 1 \mathrm{H}), 7.08$ (s, $1 \mathrm{H}), 6.90(\mathrm{~d}, J=8.4 \mathrm{~Hz}, 1 \mathrm{H}), 6.77(\mathrm{~s}, 1 \mathrm{H}), 4.28(\mathrm{~d}, J=11.8 \mathrm{~Hz}, 1 \mathrm{H}), 4.19$ (s, 1H), $3.94(\mathrm{dt}, J=14.2,7.3 \mathrm{~Hz}, 1 \mathrm{H}), 3.88-3.64(\mathrm{~m}, 2 \mathrm{H}), 1.52(\mathrm{~s}, 9 \mathrm{H}), 0.97$ (t, $J=7.2 \mathrm{~Hz}, 3 \mathrm{H})$ ppm. ${ }^{13} \mathrm{C}$ NMR (125 MHz, Chloroform- $d$ ) $\delta 174.09,165.01,152.82,138.76,129.24,127.91$, $127.72,125.47$ (q, $J=283.2 \mathrm{~Hz}), 124.86,122.81,81.07,63.32,59.43$ (q, $J=26.0 \mathrm{~Hz}), 45.83$, 44.09, 28.25, 13.26 ppm. ${ }^{19} \mathrm{~F}$ NMR (471 MHz, Chloroform- $d$ ) $\delta-73.32 \mathrm{ppm}$. ESI-HRMS m/z: $495.0738[\mathrm{M}+\mathrm{H}]^{+}, \mathrm{C}_{19} \mathrm{H}_{22} \mathrm{BrF}_{3} \mathrm{~N}_{2} \mathrm{O}_{5}+\mathrm{H}^{+}$requires 495.0736.
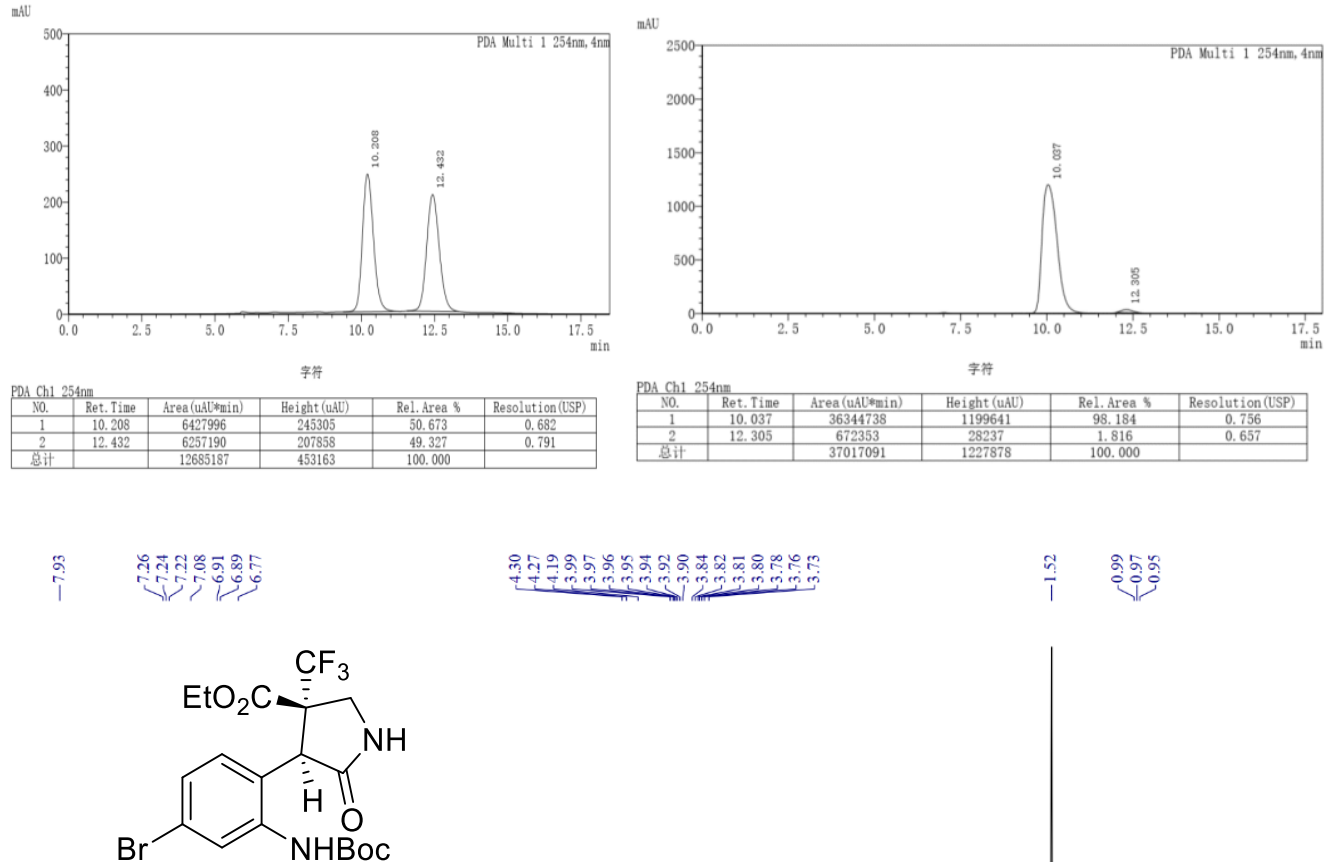

3i

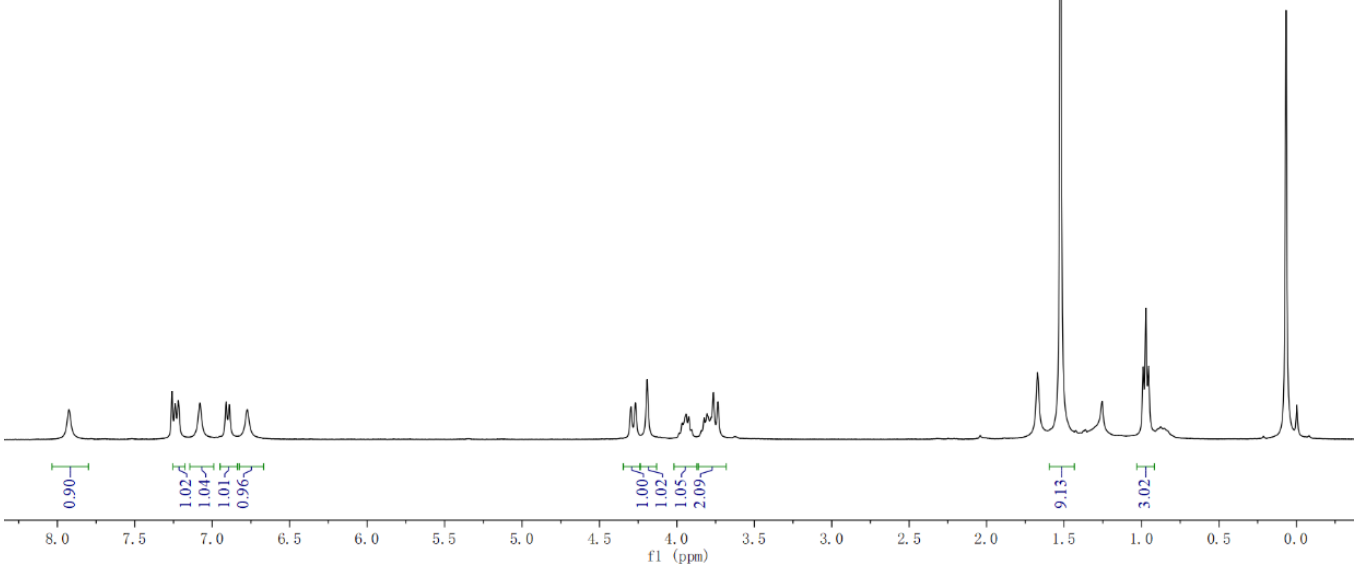



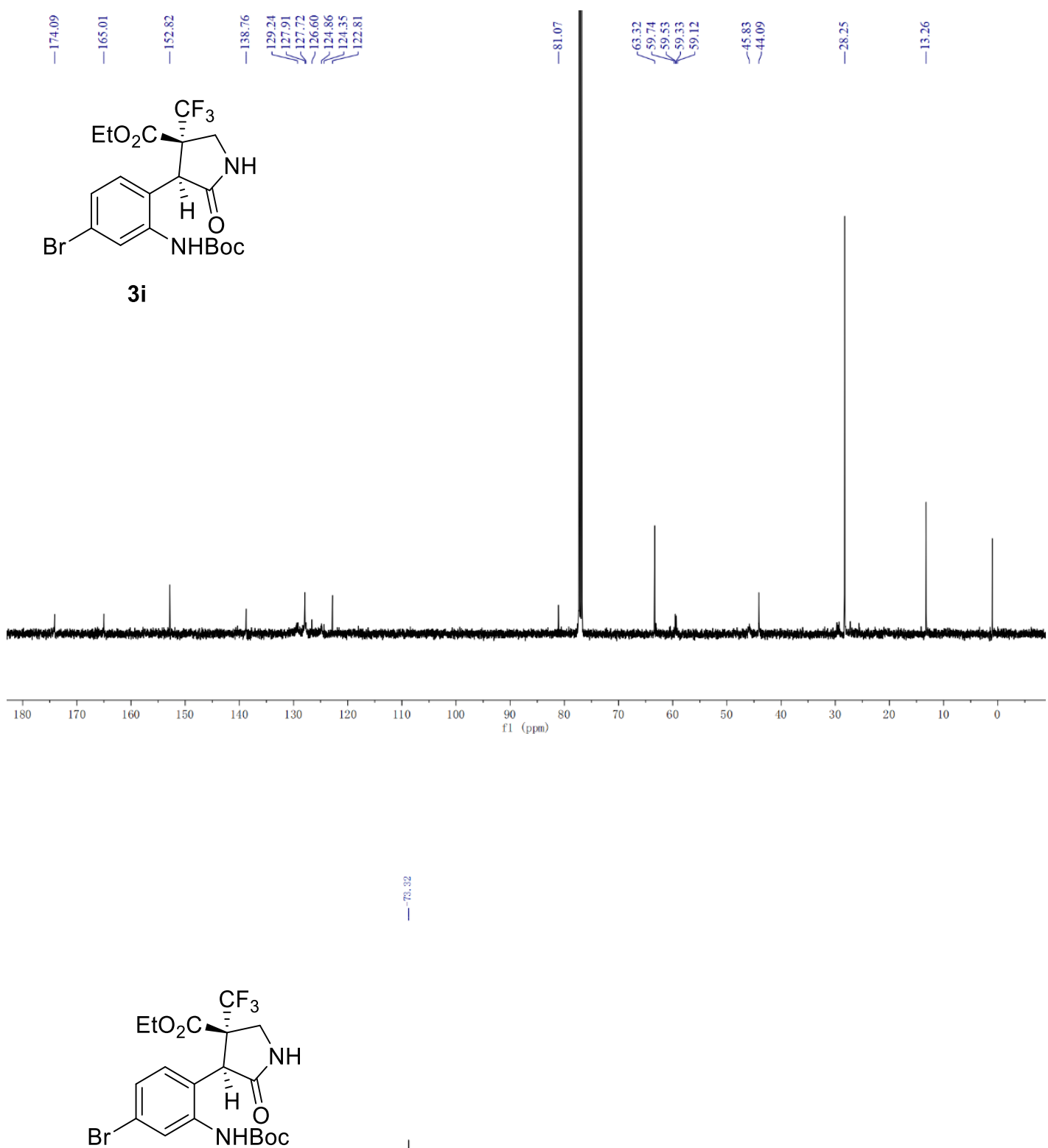

$3 \mathbf{i}$

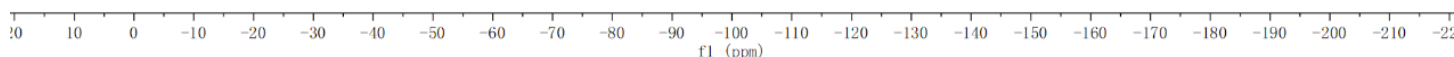


ethyl (3S,4S)-4-(2-((tert-butoxycarbonyl)amino)-3-fluorophenyl)-5-oxo-3-(trifluoromethyl)pyrrolidine-3-carboxylate (3j)<smiles>CCOC(=O)Nc1c(F)cccc1C1C(=O)NC2CNC(C(F)(F)F)C21C(F)(F)F</smiles>

3j

3j was obtained as a light yellow foam in $92 \%$ yield ( $4 \mathrm{~h}$ for Michael addition and $10 \mathrm{~h}$ for catalytic hydrogenation), $90 \%$ ee and $>20: 1 \mathrm{dr}$. The enantiomeric excess was determined by HPLC (Daicel Chiralpak AD-H, hexane/i-PrOH $=90: 10(\mathrm{v} / \mathrm{v}), \lambda=254 \mathrm{~nm}$, flow rate $\left.=0.5 \mathrm{~mL} / \mathrm{min}, 25{ }^{\circ} \mathrm{C}\right): t_{\text {minor }}=20.97 \mathrm{~min}$, $t_{\text {major }}=12.70 \mathrm{~min} ;[\alpha]_{\mathrm{D}}{ }^{25}-16.8(c \quad 0.19, \mathrm{MeOH}) ;{ }^{1} \mathrm{H}$ NMR $(500 \mathrm{MHz}$, Chloroform- $d$ ) $\delta 8.07(\mathrm{~s}, 1 \mathrm{H}), 7.18(\mathrm{q}, J=7.4 \mathrm{~Hz}, 1 \mathrm{H}), 7.06(\mathrm{t}, J=8.9 \mathrm{~Hz}, 1 \mathrm{H})$ 6.85-6.84 (m, 1H), $6.53(\mathrm{~s}, 1 \mathrm{H}), 4.51(\mathrm{~s}, 1 \mathrm{H}), 4.19(\mathrm{~d}, J=11.9 \mathrm{~Hz}, 1 \mathrm{H}), 3.92-$ $3.79(\mathrm{~m}, 1 \mathrm{H}), 3.76(\mathrm{dq}, J=14.4,7.6 \mathrm{~Hz}, 1 \mathrm{H}), 3.65(\mathrm{~d}, J=11.7 \mathrm{~Hz}, 1 \mathrm{H}), 1.47(\mathrm{~s}, 9 \mathrm{H}), 0.86(\mathrm{t}, J=$ $7.3 \mathrm{~Hz}, 3 \mathrm{H}) \mathrm{ppm} .{ }^{13} \mathrm{C}$ NMR $(125 \mathrm{MHz}$, Chloroform- $d$ ) $\delta 173.64,164.24,158.68,156.63,152.32$, 132.66, $126.94(\mathrm{~d}, J=8.6 \mathrm{~Hz}), 124.40(\mathrm{q}, J=287.3,285.0 \mathrm{~Hz}), 122.11,115.27(\mathrm{~d}, J=21.4 \mathrm{~Hz})$, $79.79,61.97,58.59$ (q, $J=26.6 \mathrm{~Hz}), 44.80,43.35,27.11,12.23 \mathrm{ppm} ;{ }^{19} \mathrm{~F}$ NMR $(471 \mathrm{MHz}$, Chloroform- $d$ ) $\delta$-73.37, -116.97 ppm. ESI-HRMS m/z: $435.1540[\mathrm{M}+\mathrm{H}]{ }^{+}, \mathrm{C}_{19} \mathrm{H}_{22} \mathrm{~F}_{4} \mathrm{~N}_{2} \mathrm{O}_{5}+\mathrm{H}^{+}$ requires 435.1538 .
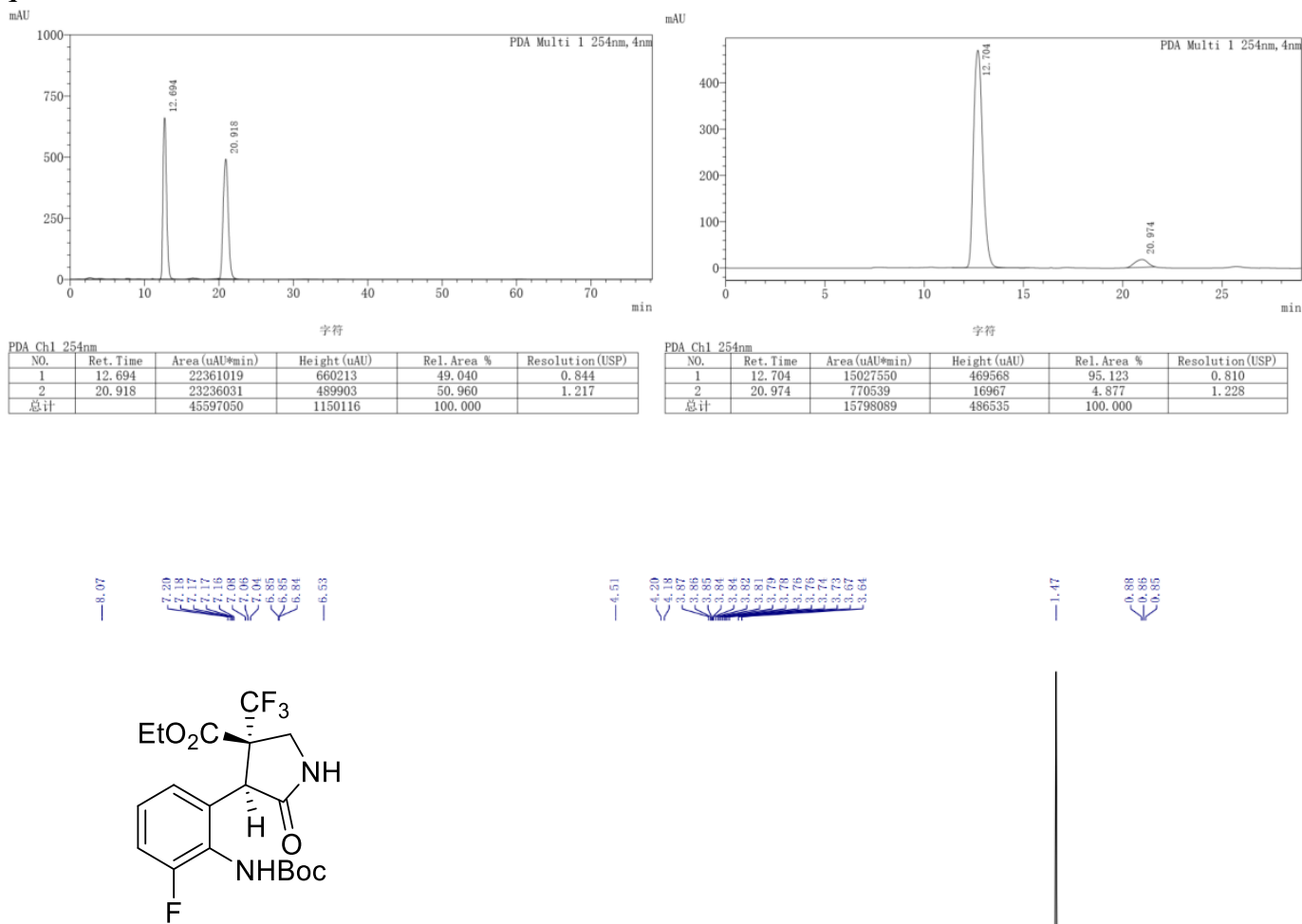

$3 \mathbf{j}$

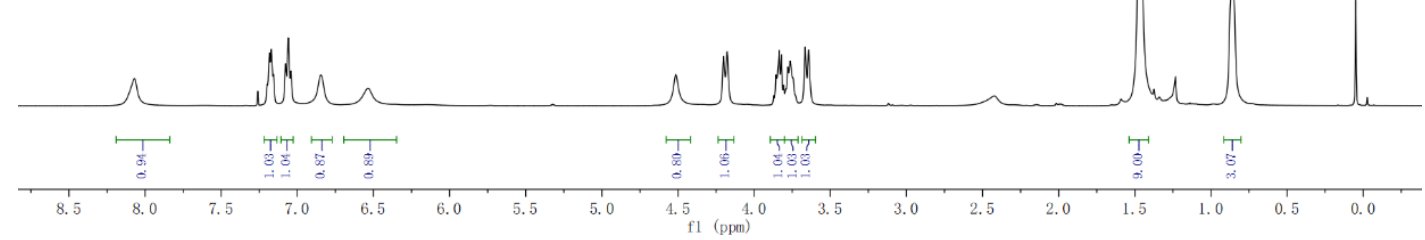




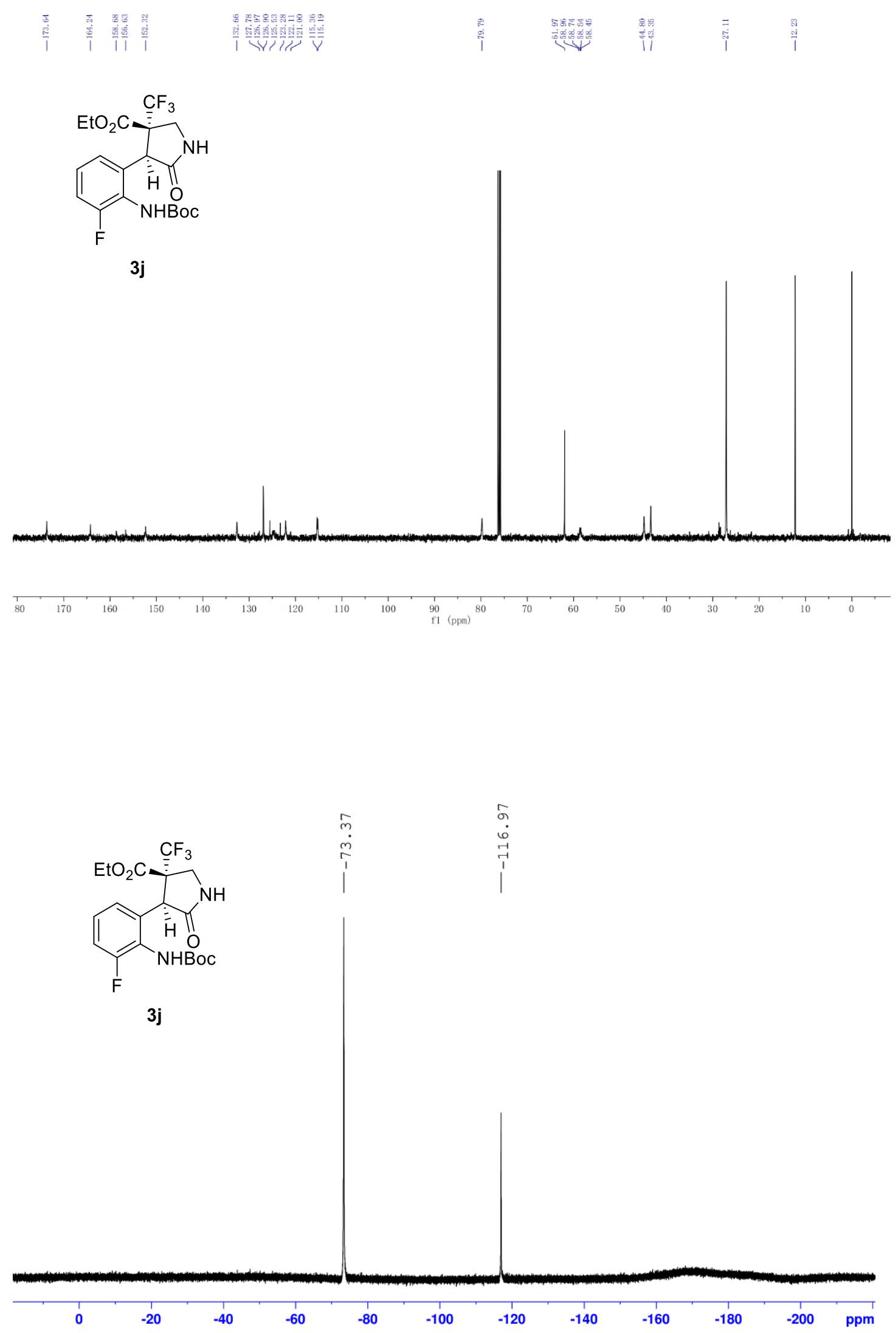


(3S,4S)-4-(2-((tert-butoxycarbonyl)amino)-3-chlorophenyl)-5-oxo-3-(trifluoromethyl)pyrrolidine-3-carboxylate (3k)<smiles>CCOC(=O)Nc1c(Cl)cccc1C1C(=O)NCC1C(F)(F)F</smiles>

$3 k$

3k was obtained as a light yellow foam in $90 \%$ yield ( $4 \mathrm{~h}$ for Michael addition and $11 \mathrm{~h}$ for catalytic hydrogenation), $94 \%$ ee and $>20: 1 \mathrm{dr}$. The enantiomeric excess was determined by HPLC (Daicel Chiralpak AD-H, hexane/i-PrOH $=80: 20(\mathrm{v} / \mathrm{v}), \lambda=254 \mathrm{~nm}$, flow rate $\left.=1.0 \mathrm{~mL} / \mathrm{min}, 25{ }^{\circ} \mathrm{C}\right): t_{\text {minor }}=4.25 \mathrm{~min}$, $\left.t_{\text {major }}=4.74 \mathrm{~min} ;[\alpha]_{\mathrm{D}}{ }^{25}-16.9(c) 0.23, \mathrm{MeOH}\right) ;{ }^{1} \mathrm{H} \mathrm{NMR}(500 \mathrm{MHz}$, Chloroform- $d$ ) $\delta 8.10(\mathrm{~s}, 1 \mathrm{H}), 7.39(\mathrm{~d}, J=8.0 \mathrm{~Hz}, 1 \mathrm{H}), 7.17(\mathrm{t}, J=7.9 \mathrm{~Hz}, 1 \mathrm{H})$, $7.00(\mathrm{~d}, J=7.8 \mathrm{~Hz}, 1 \mathrm{H}), 6.44(\mathrm{~s}, 1 \mathrm{H}), 4.57(\mathrm{~s}, 1 \mathrm{H}), 4.19(\mathrm{~d}, J=11.9 \mathrm{~Hz}, 1 \mathrm{H})$, $3.88-3.71(\mathrm{~m}, 2 \mathrm{H}), 3.68(\mathrm{~d}, J=11.8 \mathrm{~Hz}, 1 \mathrm{H}), 1.48(\mathrm{~s}, 9 \mathrm{H}), 0.86(\mathrm{t}, J=7.1 \mathrm{~Hz}, 3 \mathrm{H}) \mathrm{ppm} .{ }^{13} \mathrm{C}$ NMR (125 MHz, Chloroform- $d$ ) $\delta 174.66,165.24,153.23,134.88,134.32,129.96,128.80,128.13$, $126.69,125.41(\mathrm{q}, J=283.2 \mathrm{~Hz}), 80.75,62.89,59.63(\mathrm{q}, J=26.7 \mathrm{~Hz}), 46.76,44.42,28.14,13.23$ ppm; ${ }^{19} \mathrm{~F}$ NMR (471 MHz, Chloroform- $d$ ) $\delta-73.26$ ppm. ESI-HRMS m/z: $451.1237[\mathrm{M}+\mathrm{H}]{ }^{+}$, $\mathrm{C}_{19} \mathrm{H}_{22} \mathrm{ClF}_{3} \mathrm{~N}_{2} \mathrm{O}_{5}+\mathrm{H}^{+}$requires 451.1242 .
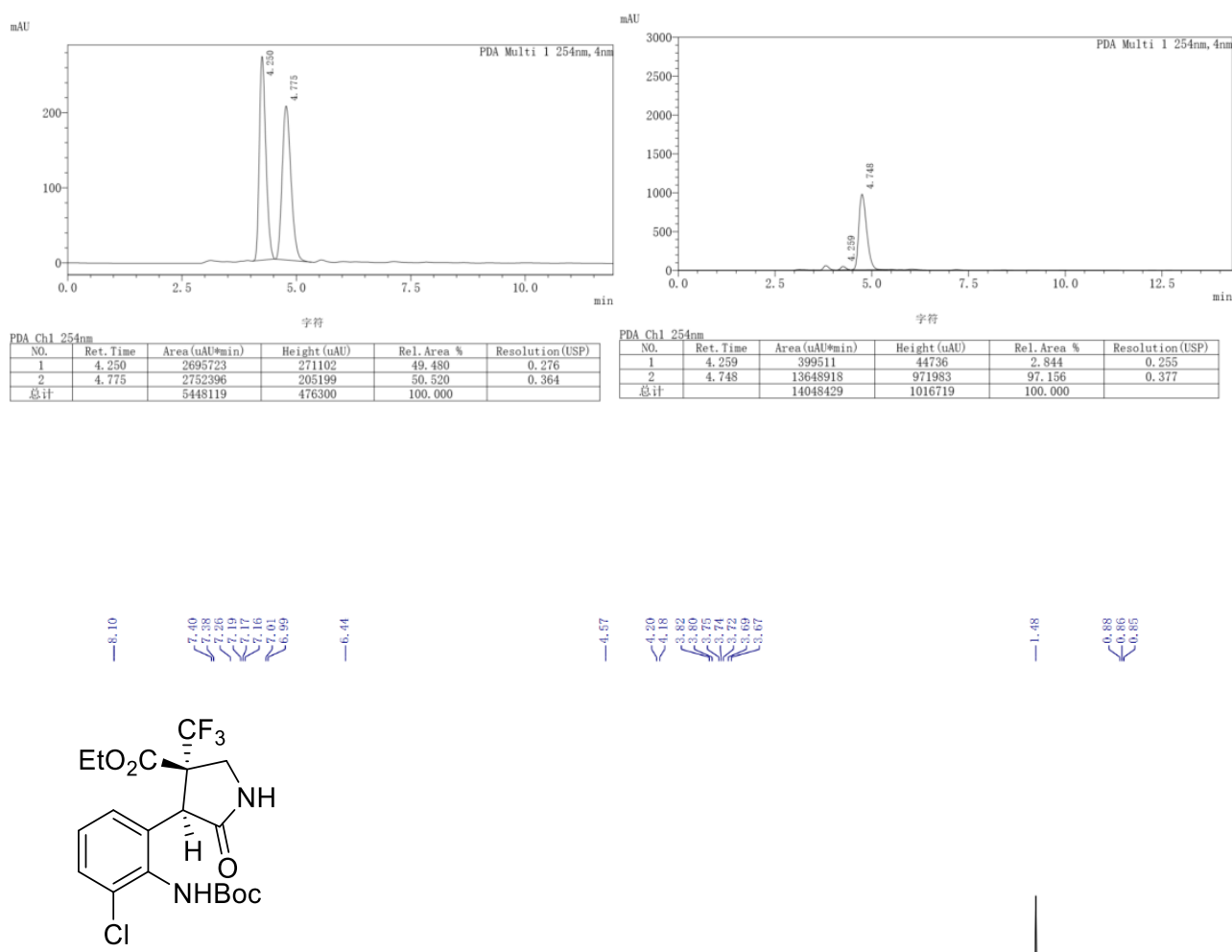

$3 k$

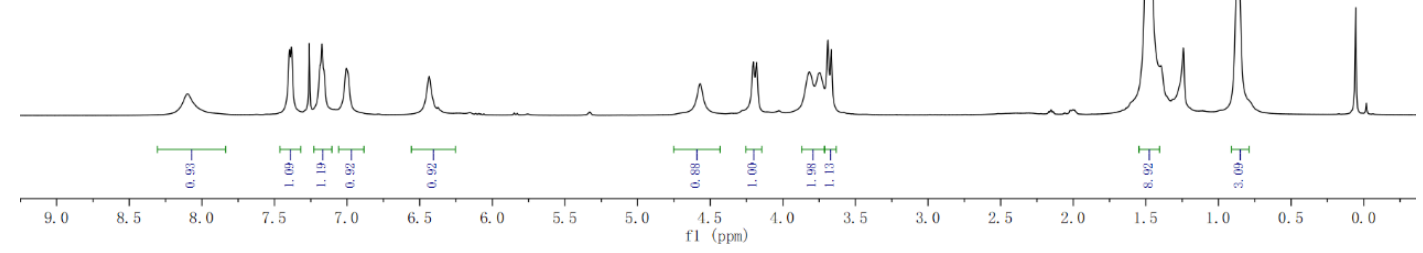




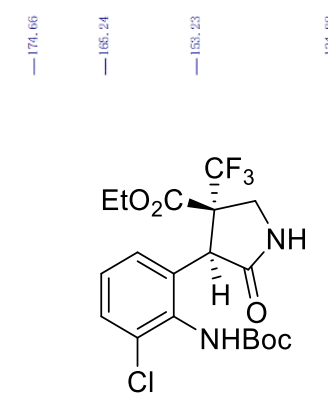

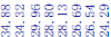

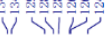

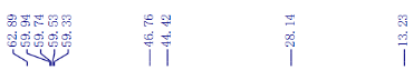

3k
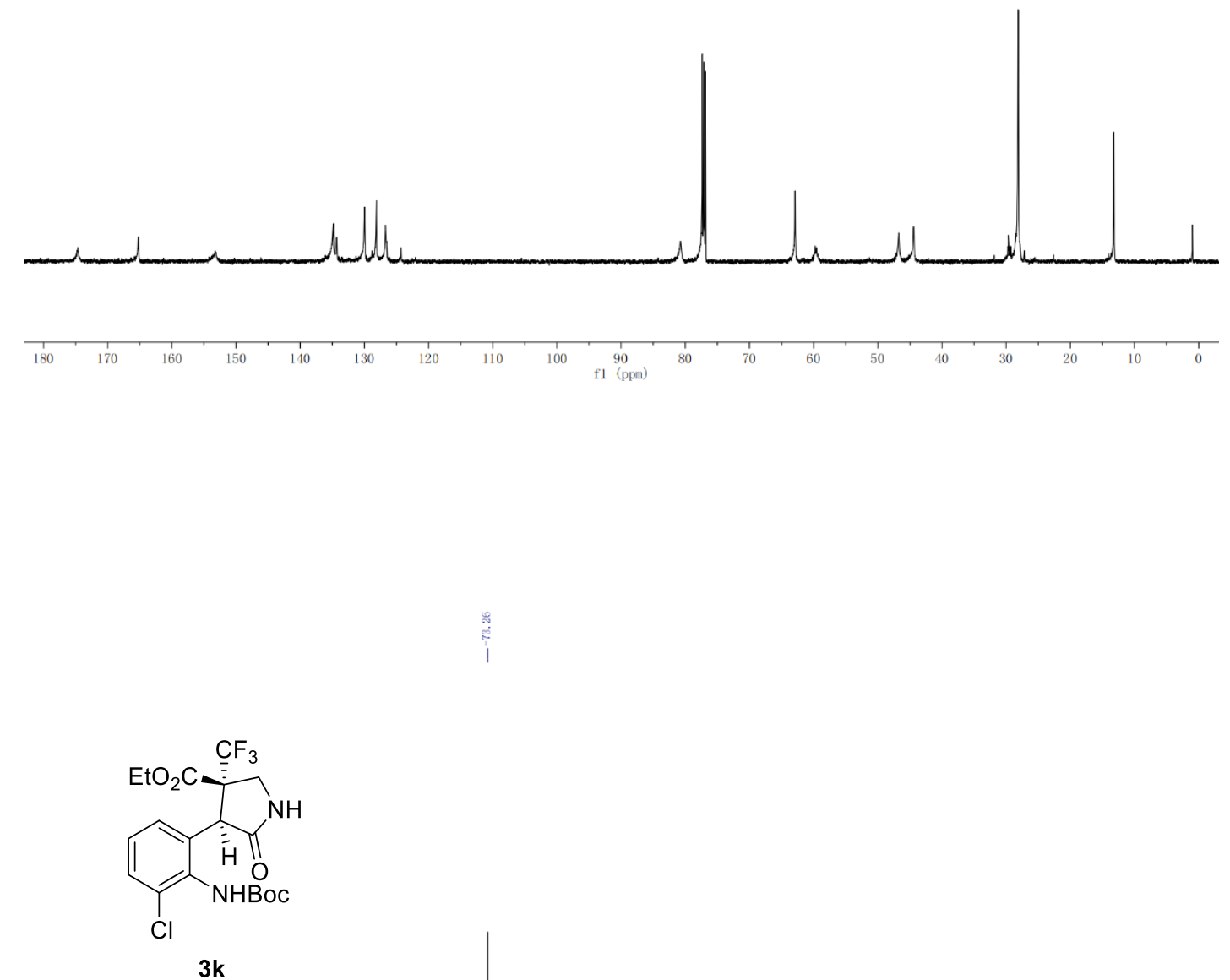
(3S,4S)-4-(3-bromo-2-((tert-butoxycarbonyl)amino)phenyl)-5-oxo-3-(trifluoromethyl)pyrrolidine-3-carboxylate (3l)<smiles>CCOC(=O)Nc1c(Br)cccc1C1C(=O)NC2CC(C(F)(F)F)CC21C(F)(F)F</smiles>

3!

31 was obtained as a yellow foam in $91 \%$ yield ( $4 \mathrm{~h}$ for Michael addition and $10 \mathrm{~h}$ for catalytic hydrogenation), $87 \%$ ee and $>20: 1 \mathrm{dr}$. The enantiomeric excess was determined by HPLC (Daicel Chiralpak AD-H, hexane/i-PrOH $=90: 10(\mathrm{v} / \mathrm{v}), \lambda=254 \mathrm{~nm}$, flow rate $\left.=0.5 \mathrm{~mL} / \mathrm{min}, 25^{\circ} \mathrm{C}\right): t_{\text {minor }}=17.77 \mathrm{~min}$, $t_{\text {major }}=10.25 \mathrm{~min} ;[\alpha]_{\mathrm{D}}{ }^{25}-25.1(c 0.34, \mathrm{MeOH}) ;{ }^{1} \mathrm{H} \mathrm{NMR}(500 \mathrm{MHz}$, Chloroform- $d$ ) $\delta 8.02(\mathrm{~s}, 1 \mathrm{H}), 7.18(\mathrm{td}, J=8.1,5.3 \mathrm{~Hz}, 1 \mathrm{H}), 7.06(\mathrm{t}, J=8.8 \mathrm{~Hz}$ $1 \mathrm{H}), 6.86-6.85(\mathrm{~m}, 1 \mathrm{H}), 6.51(\mathrm{~s}, 1 \mathrm{H}), 4.51(\mathrm{~s}, 1 \mathrm{H}), 4.20(\mathrm{~d}, J=11.8 \mathrm{~Hz}, 1 \mathrm{H})$, $3.84(\mathrm{dq}, J=10.4,7.1 \mathrm{~Hz}, 1 \mathrm{H}), 3.77(\mathrm{p}, J=7.1 \mathrm{~Hz}, 1 \mathrm{H}), 3.66(\mathrm{~d}, J=11.8 \mathrm{~Hz}, 1 \mathrm{H}), 1.47(\mathrm{~s}, 9 \mathrm{H})$, 0.87 (t, $J=7.2 \mathrm{~Hz}, 3 \mathrm{H}) \mathrm{ppm} .{ }^{13} \mathrm{C}$ NMR (125 MHz, Chloroform- $d$ ) $\delta 174.56,165.21,153.17$, $136.28,135.09,133.18,128.59,127.46,125.41$ (q, $J=282.4 \mathrm{~Hz}), 125.06,80.83,62.91,59.68$ (q, $J=26.0 \mathrm{~Hz}), 47.02,44.39,28.18,13.28 \mathrm{ppm} ;{ }^{19} \mathrm{~F}$ NMR (471 MHz, Chloroform- $d$ ) $\delta-73.22 \mathrm{ppm}$. ESI-HRMS m/z: $495.0734[\mathrm{M}+\mathrm{H}]{ }^{+}, \mathrm{C}_{19} \mathrm{H}_{22} \mathrm{BrF}_{3} \mathrm{~N}_{2} \mathrm{O}_{5}+\mathrm{H}^{+}$requires 495.0736.
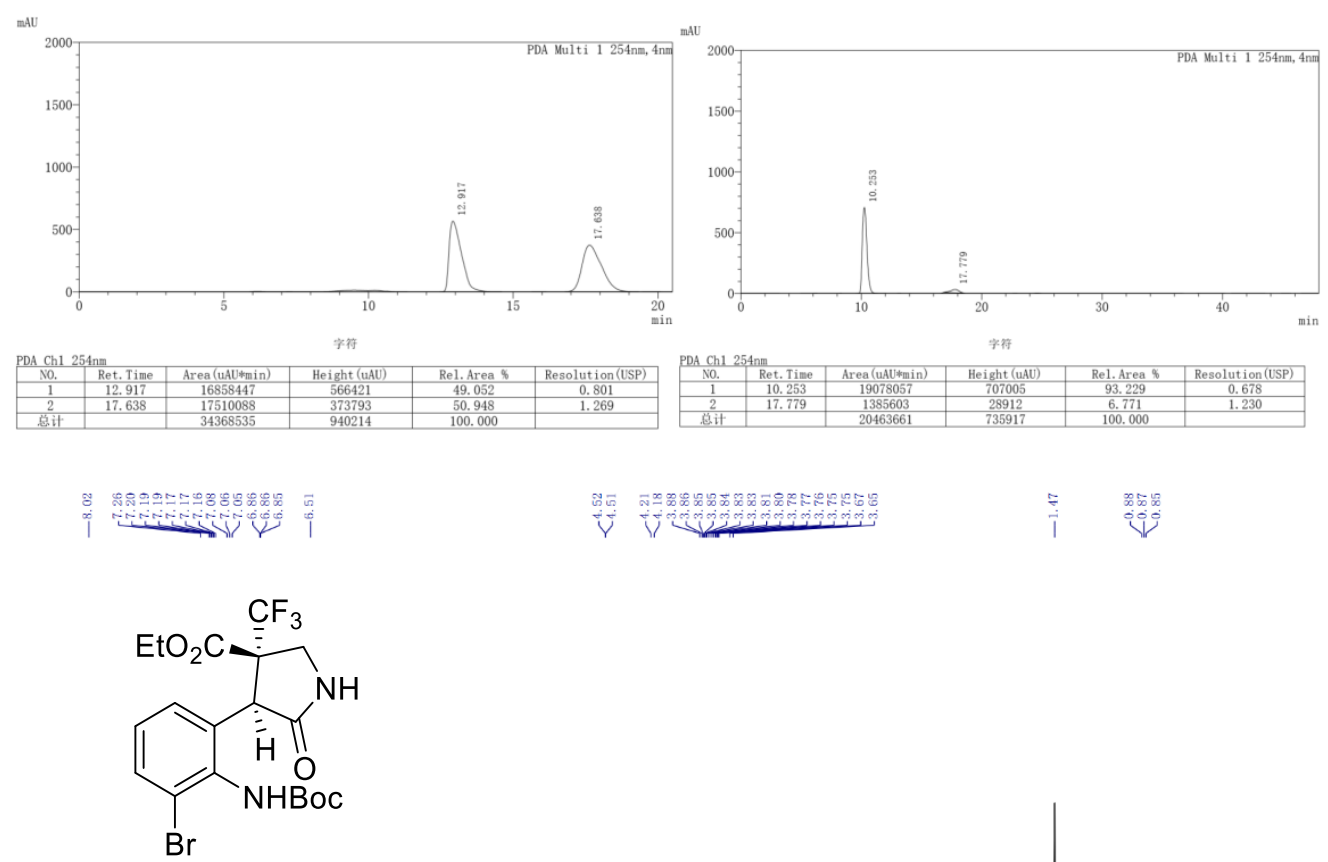

31

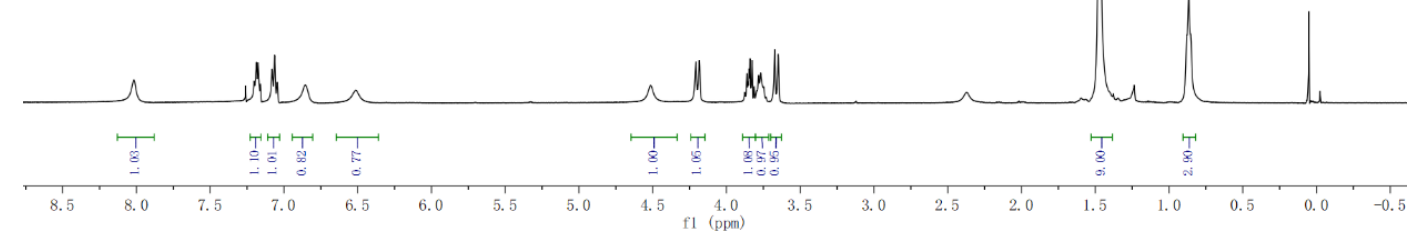




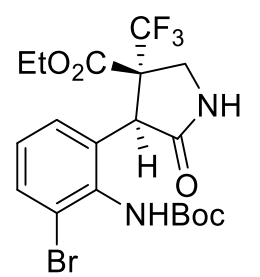

3I
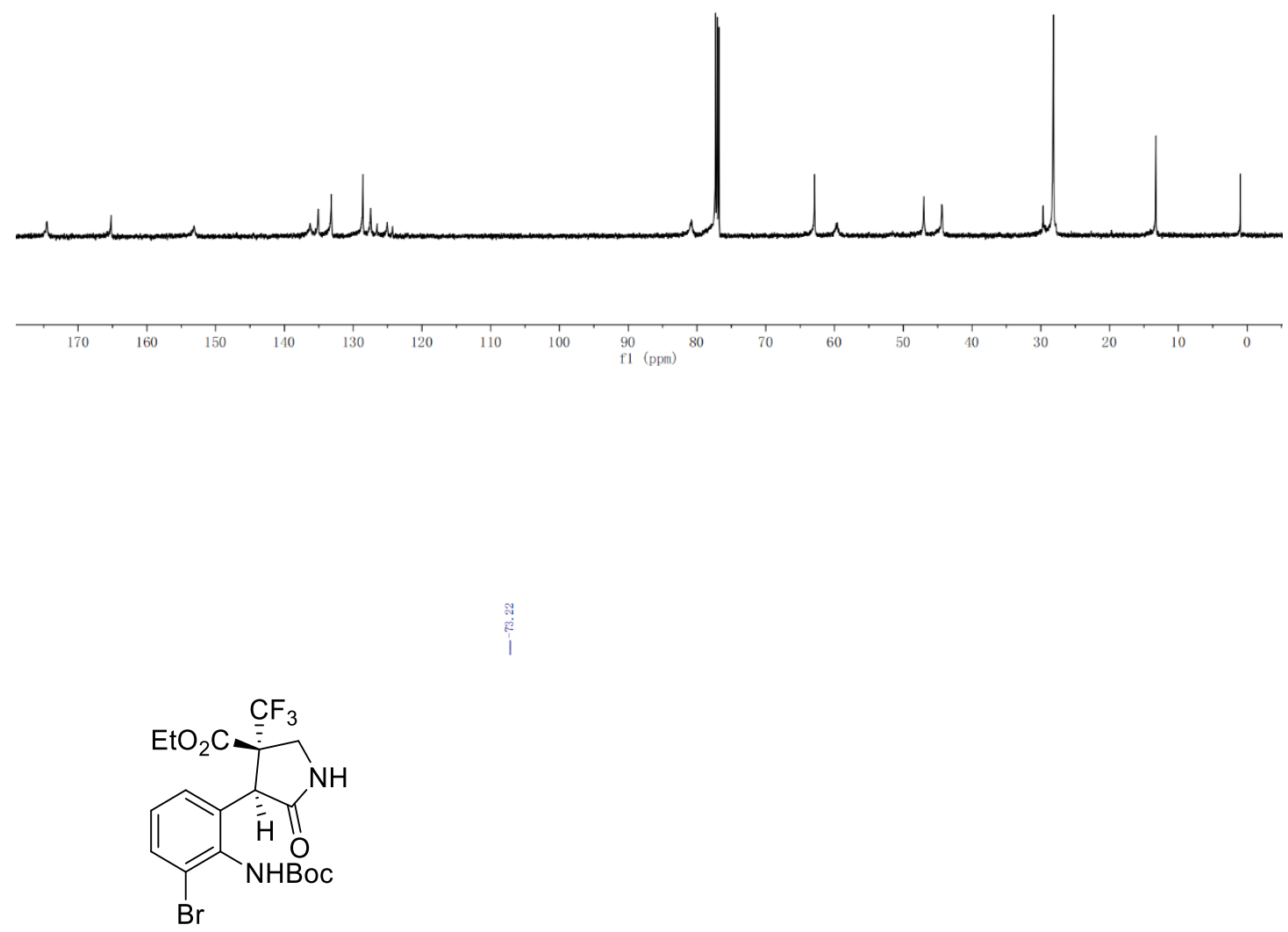

3I

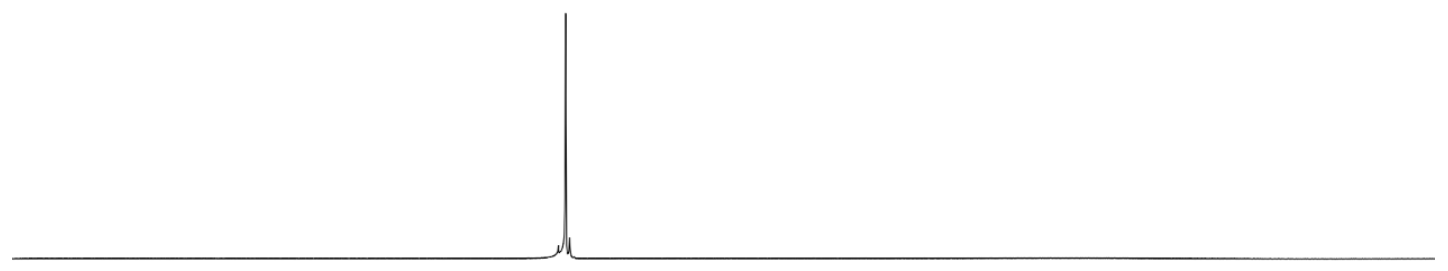

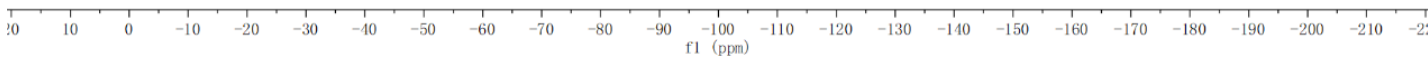


<smiles>CCOC(=O)C(=C1C(=O)N(C(=O)OC(C)(C)C)c2c(Br)cccc21)C(F)(F)F</smiles><smiles>CCOC(=O)C(CN(C)C(=O)[O-])(C1C(=O)Nc2c(Br)cccc21)C(F)(F)F</smiles>

To a sample bottle equipped with a magnetic stirring bar, C10 (10 mol\%) and 2a (2 mmol, 20.0 equiv) were added at room temperature under $\mathrm{N}_{2}$. Subsequently, $1 \mathbf{l}(0.1 \mathrm{mmol}, 1.0$ equiv) was added in one portion. After completion (monitored by TLC), the reaction mixture was directly concentrated under reduced pressure. The residue was dissolved in DCM $(0.1 \mathrm{ml}), \mathrm{CF}_{3} \mathrm{COOH}(0.5$ mmol, 5.0 equiv.) was added. After $0.5 \mathrm{~h}$, the mixture was concentrated and the residue was purified by flash chromatography on silica gel (petroleum ether/ethyl acetate) to afford the compounds 31'.<smiles>CCOC(=O)C(C1C(=O)Nc2c(Br)cccc21)(C(F)(F)F)C(F)(F)F</smiles>

31' was obtained as a yellow foam in $97 \%$ yield ( $4 \mathrm{~h}$ for Michael addition and $0.5 \mathrm{~h}$ for removing the Boc group), $87 \%$ ee (the ee value was measured after removing $\mathrm{Br}$ group by hydrogenation) and 3.5:1 dr. The enantiomeric excess was determined by HPLC (Daicel Chiralpak AD-H, hexane/i-PrOH $=80: 20(\mathrm{v} / \mathrm{v}), \lambda=254 \mathrm{~nm}$, flow rate $\left.=1.0 \mathrm{~mL} / \mathrm{min}, 25^{\circ} \mathrm{C}\right): t_{\text {minor }}=7.22 \mathrm{~min}$, $t_{\text {major }}=9.10 \mathrm{~min} ;[\alpha]_{\mathrm{D}}{ }^{25} 41.3($ c $0.82, \mathrm{MeOH}) ;{ }^{1} \mathrm{H}$ NMR $(400 \mathrm{MHz}$, Chloroform- $d$ ) $\delta 8.85(\mathrm{~s}, 1 \mathrm{H}), 7.43(\mathrm{t}, J=8.4 \mathrm{~Hz}, 2 \mathrm{H}), 6.95(\mathrm{t}, J=8.0 \mathrm{~Hz}, 1 \mathrm{H}), 5.60(\mathrm{~d}, J=15.4$ $\mathrm{Hz}, 1 \mathrm{H}), 5.32$ (d, $J=15.5 \mathrm{~Hz}, 1 \mathrm{H}), 4.62(\mathrm{~s}, 1 \mathrm{H}), 4.49$ (qd, $J=7.2,1.8 \mathrm{~Hz}, 2 \mathrm{H}), 1.40$ (t, $J=7.1 \mathrm{~Hz}$, $3 \mathrm{H}) \mathrm{ppm} .{ }^{13} \mathrm{C}$ NMR (125 MHz, Chloroform- $d$ ) $\delta 173.85,165.18,140.49,132.00,126.19,124.77$, $123.91,123.57$ (q, $J=286.8 \mathrm{~Hz}), 103.15,72.66,64.14,57.74$ (q, $J=24.8 \mathrm{~Hz}), 46.30,13.79$ ppm; ${ }^{19} \mathrm{~F}$ NMR (471 MHz, Chloroform- $d$ ) $\delta$-64.66 ppm. ESI-HRMS m/z: $424.9954[\mathrm{M}+\mathrm{H}]{ }^{+}$, $\mathrm{C}_{14} \mathrm{H}_{12} \mathrm{BrF}_{3} \mathrm{~N}_{2} \mathrm{O}_{5}+\mathrm{H}^{+}$requires 424.9954 .
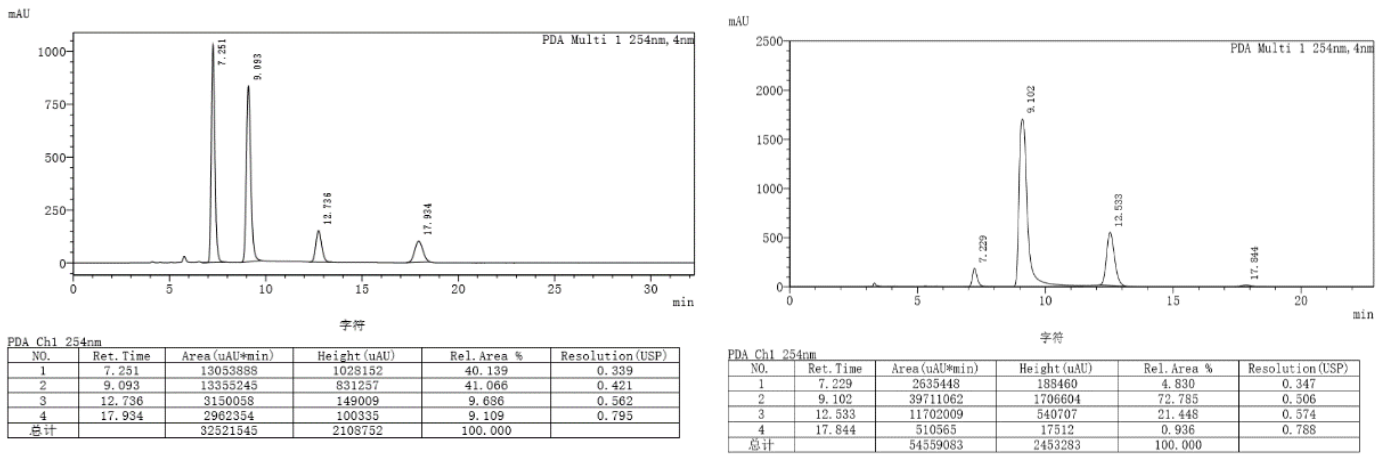
<smiles>CCOC(=O)C(CN[N+](=O)[O-])(C1C(=O)Nc2c(Br)cccc21)C(F)(F)F</smiles>

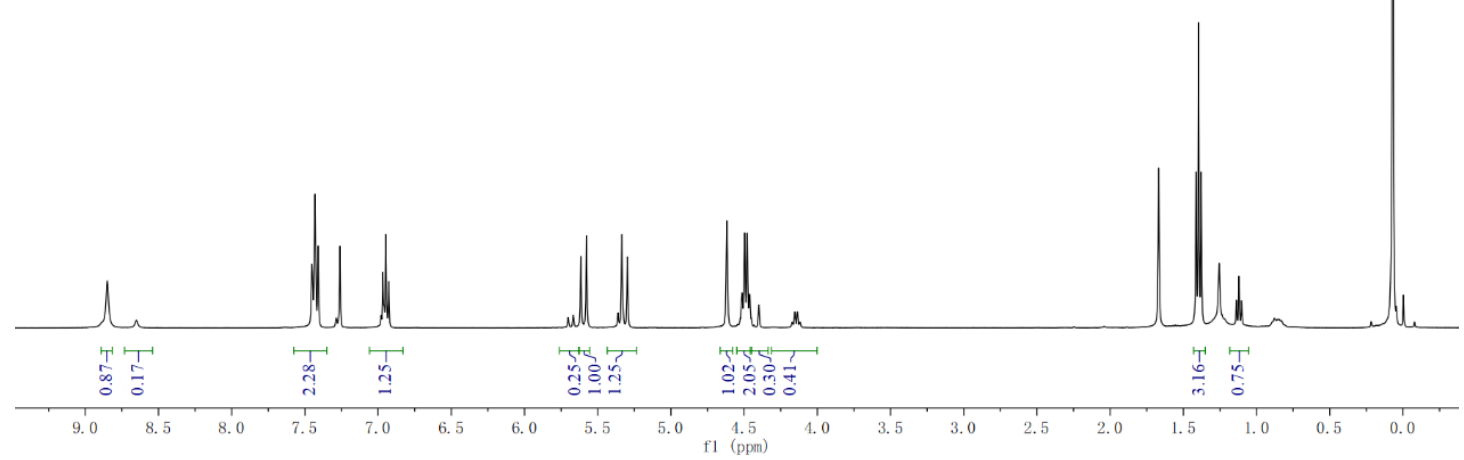

$$
\text { (1) }
$$
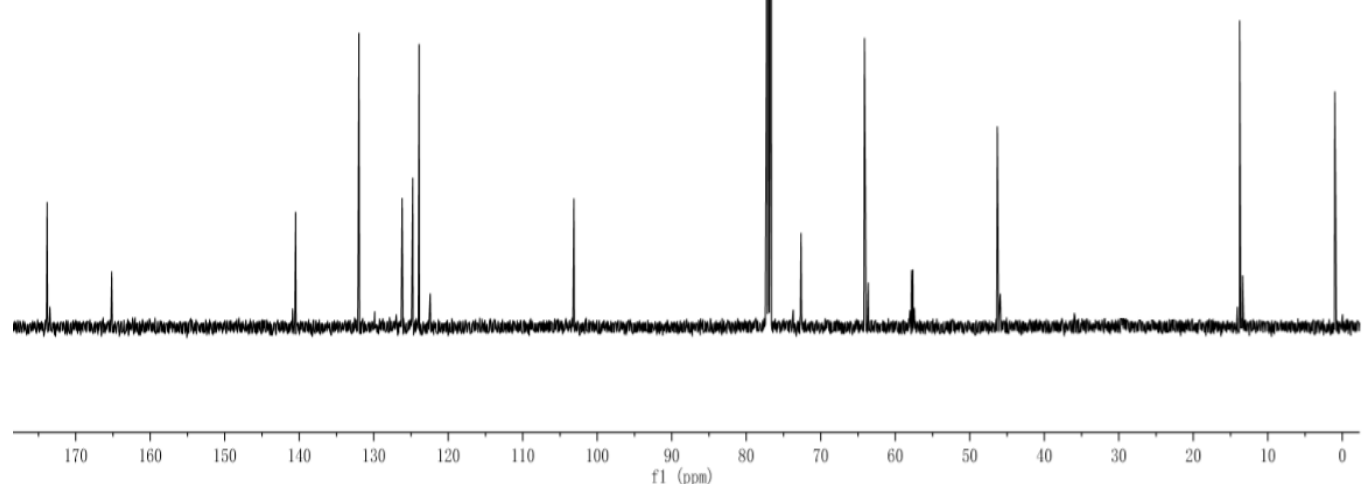
<smiles>CCOC(=O)C(C(C(=O)Nc1ccccc1)C(=O)Nc1cccc(Br)c1)C(F)(F)F</smiles>

$31^{\prime}$

ethyl

(3S,4S)-4-(2-((tert-butoxycarbonyl)amino)-3,5-dimethylphenyl)-5-oxo-3-(trifluoromethyl)pyrroli dine-3-carboxylate (3m)<smiles>CCOC(=O)[O-]</smiles>

$3 m$

$3 \mathbf{m}$ was obtained as a yellow foam in $90 \%$ yield ( $4 \mathrm{~h}$ for Michael addition and $12 \mathrm{~h}$ for catalytic hydrogenation), $90 \%$ ee and $>20: 1 \mathrm{dr}$. The enantiomeric excess was determined by HPLC (Daicel Chiralpak OD-H, hexane $/ \mathrm{i}-\mathrm{PrOH}=90: 10(\mathrm{v} / \mathrm{v}), \lambda=254 \mathrm{~nm}$, flow rate $\left.=0.5 \mathrm{~mL} / \mathrm{min}, 25^{\circ} \mathrm{C}\right)$ : $t_{\text {minor }}=16.95 \mathrm{~min}, t_{\text {major }}=9.74 \mathrm{~min} ;[\alpha]_{\mathrm{D}} 2532.56(c 0.58, \mathrm{MeOH}) ;{ }^{1} \mathrm{H}$ NMR (500 MHz, Chloroform- $d$ ) $\delta 7.99(\mathrm{~s}, 1 \mathrm{H}), 6.98(\mathrm{~s}, 1 \mathrm{H}), 6.68(\mathrm{~s}, 1 \mathrm{H})$, $6.35(\mathrm{~s}, 1 \mathrm{H}), 4.40(\mathrm{~s}, 1 \mathrm{H}), 4.24(\mathrm{~d}, J=12.0 \mathrm{~Hz}, 1 \mathrm{H}), 3.78(\mathrm{~m}, 2 \mathrm{H}), 3.66$ $(\mathrm{d}, J=11.9 \mathrm{~Hz}, 1 \mathrm{H}), 2.24(\mathrm{~d}, J=2.9 \mathrm{~Hz}, 6 \mathrm{H}), 1.48(\mathrm{~s}, 9 \mathrm{H}), 0.83(\mathrm{t}, J=7.1 \mathrm{~Hz}, 3 \mathrm{H}) \mathrm{ppm} .{ }^{13} \mathrm{C}$ NMR (125 MHz, Chloroform- $d$ ) $\delta 175.39,165.28,153.39,137.61,137.06,132.89,132.00,131.66$, 125.61 (q, $J=282.1 \mathrm{~Hz}), 125.48,79.75,62.73,59.73$ (q, $J=25.6 \mathrm{~Hz}), 46.38,44.26,28.25,20.95$, 18.50, $13.11 \mathrm{ppm} ;{ }^{19} \mathrm{~F}$ NMR (471 MHz, Chloroform- $d$ ) $\delta-73.69$ ppm. ESI-HRMS m/z: 445.1942 $[\mathrm{M}+\mathrm{H}]^{+}, \mathrm{C}_{21} \mathrm{H}_{27} \mathrm{~F}_{3} \mathrm{~N}_{2} \mathrm{O}_{5}+\mathrm{H}^{+}$requires 445.1945.
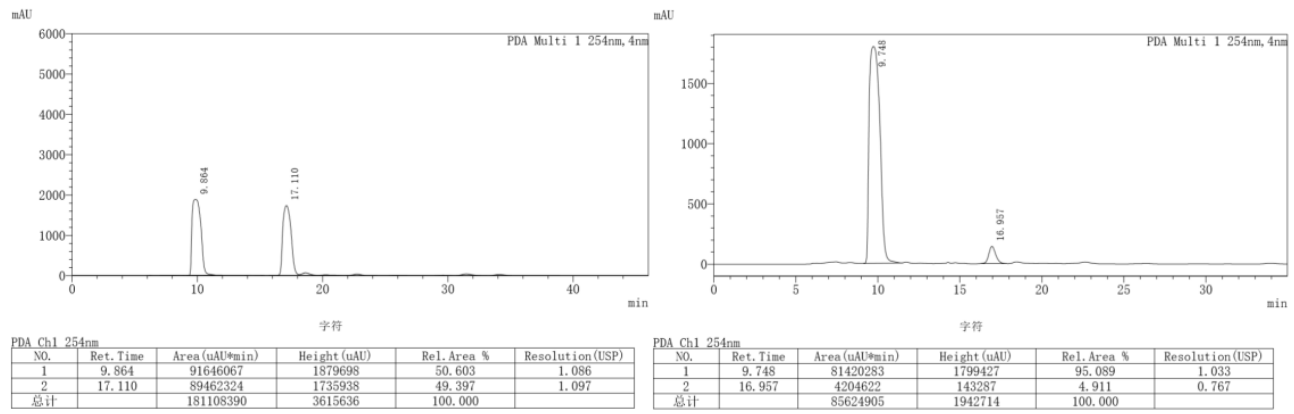

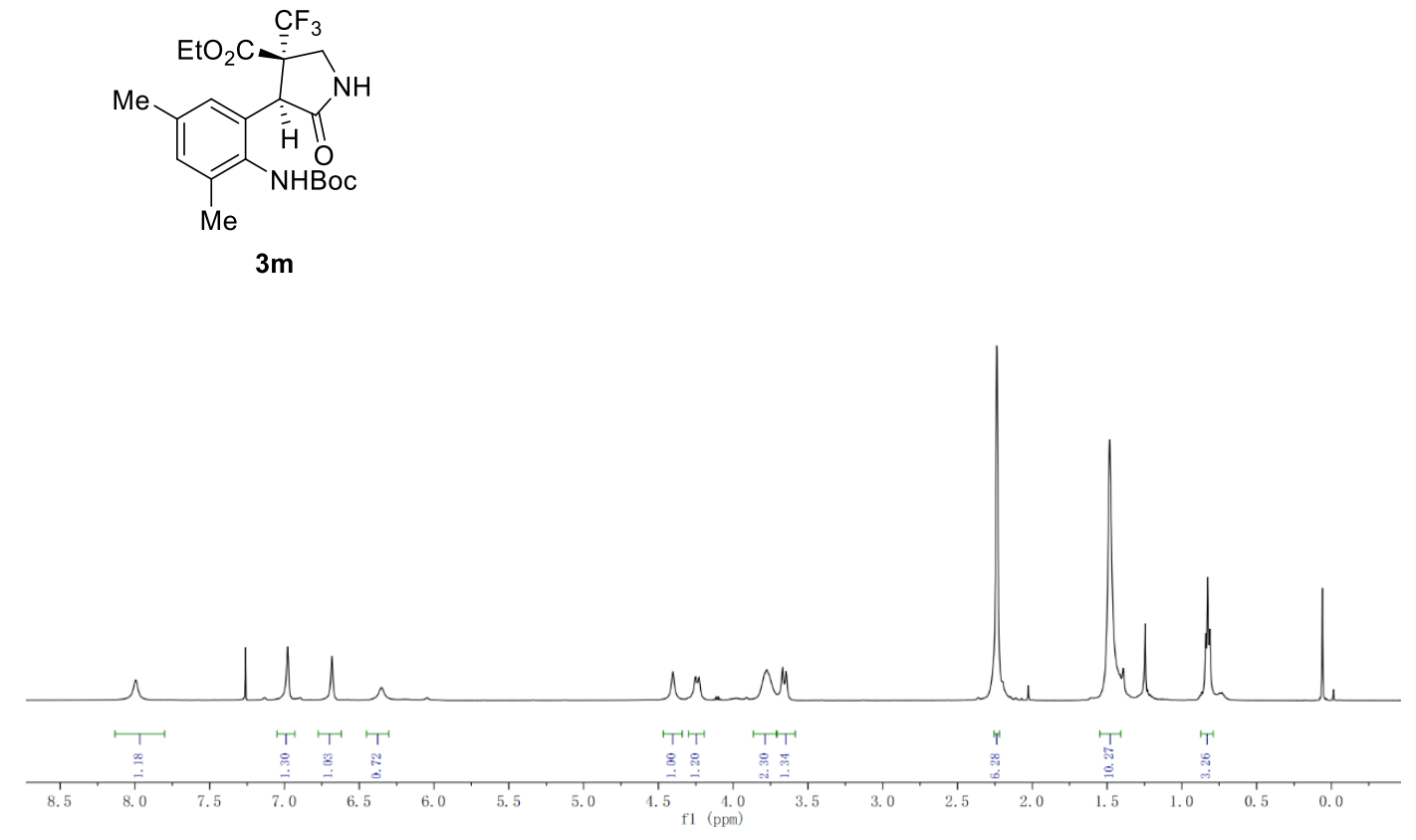

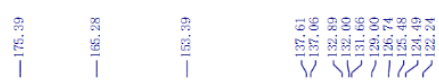

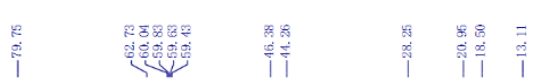

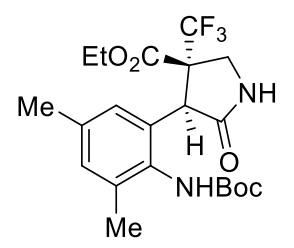

$3 m$

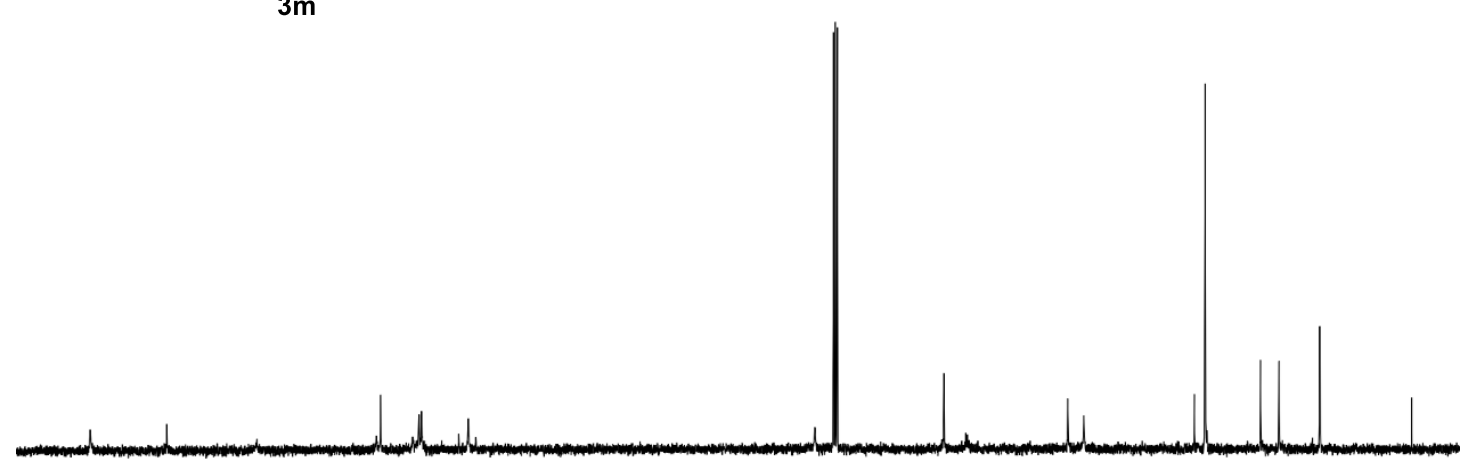

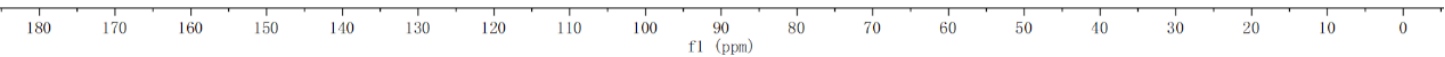



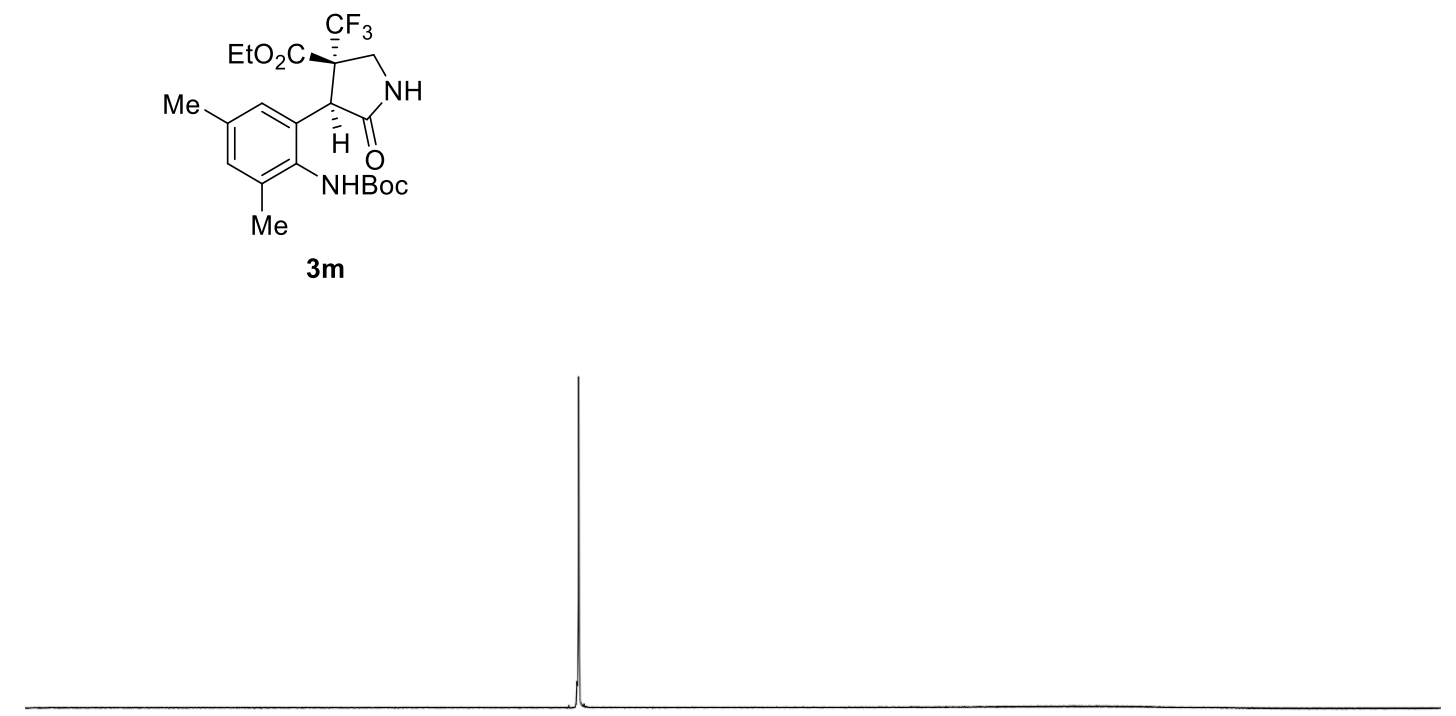

ethyl

(3S,4S)-4-(2-((tert-butoxycarbonyl)amino)pyridin-3-yl)-5-oxo-3-(trifluoromethyl)pyrrolidine-3-c arboxylate (3n)<smiles></smiles>

3n was obtained as a yellow foam in $90 \%$ yield ( $5 \mathrm{~h}$ for Michael addition and $12 \mathrm{~h}$ for catalytic hydrogenation), $85 \%$ ee and $>20: 1 \mathrm{dr}$. The enantiomeric excess was determined by HPLC (Daicel Chiralpak AD-H, hexane/i-PrOH $=80: 20(\mathrm{v} / \mathrm{v}), \lambda=254 \mathrm{~nm}$, flow rate $\left.=1.0 \mathrm{~mL} / \mathrm{min}, 25^{\circ} \mathrm{C}\right): t_{\text {minor }}=9.86 \mathrm{~min}$, $t_{\text {major }}=5.41 \mathrm{~min} ;[\alpha]_{\mathrm{D}}{ }^{25} 27.15(c \quad 0.21, \mathrm{MeOH}) ;{ }^{1} \mathrm{H} \mathrm{NMR}(500 \mathrm{MHz}$,

$3 n$ Chloroform- $d$ ) $\delta 8.42(\mathrm{~s}, 1 \mathrm{H}), 7.79(\mathrm{~s}, 1 \mathrm{H}), 7.45(\mathrm{~d}, J=7.8 \mathrm{~Hz}, 1 \mathrm{H}), 7.25(\mathrm{~d}, J$ $=7.8 \mathrm{~Hz}, 1 \mathrm{H}), 7.10(\mathrm{t}, J=6.3 \mathrm{~Hz}, 1 \mathrm{H}), 4.65(\mathrm{~s}, 1 \mathrm{H}), 4.18(\mathrm{~d}, J=11.5 \mathrm{~Hz}, 1 \mathrm{H})$, $3.87(\mathrm{dt}, J=14.1,7.0 \mathrm{~Hz}, 1 \mathrm{H}), 3.73(\mathrm{~m}, 2 \mathrm{H}), 1.51(\mathrm{~s}, 9 \mathrm{H}), 0.93(\mathrm{t}, J=7.2 \mathrm{~Hz}, 3 \mathrm{H}) \mathrm{ppm} .{ }^{13} \mathrm{C} \mathrm{NMR}$ $(125 \mathrm{MHz}$, Chloroform- $d$ ) $\delta 173.93,165.36,152.61,150.72,148.63,138.67,125.30$ (q, $J=282.0$ $\mathrm{Hz}), 123.18,120.74,81.11,63.02,59.52(\mathrm{q}, J=26.5 \mathrm{~Hz}), 46.48,44.37,28.19,13.37 \mathrm{ppm} ;{ }^{19} \mathrm{~F}$ NMR (471 MHz, Chloroform- $d$ ) $\delta-72.65 \mathrm{ppm}$. ESI-HRMS m/z: $418.1582[\mathrm{M}+\mathrm{H}]{ }^{+}$, $\mathrm{C}_{18} \mathrm{H}_{22} \mathrm{~F}_{3} \mathrm{~N}_{3} \mathrm{O}_{5}+\mathrm{H}^{+}$requires 418.1584 .
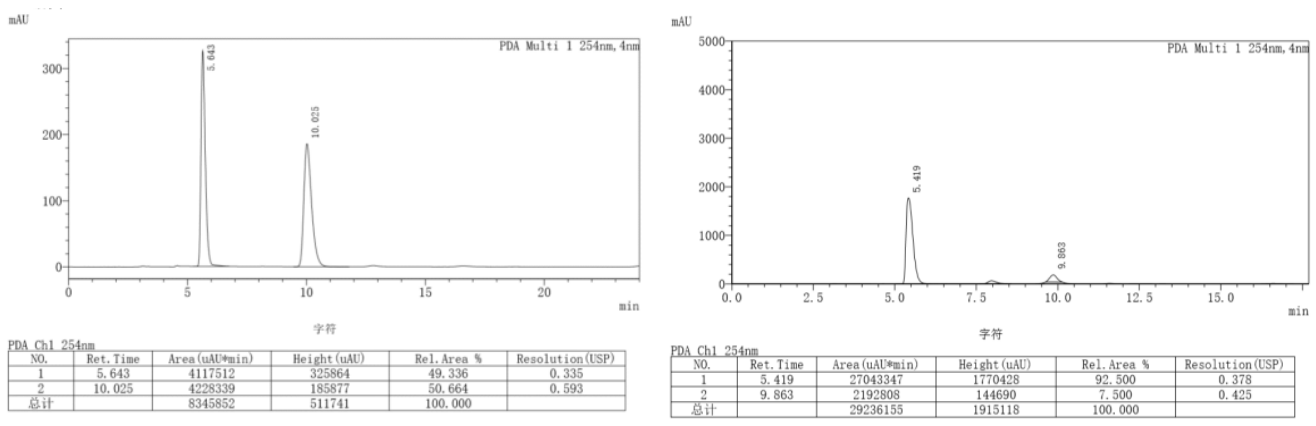


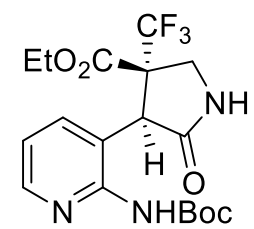

$3 n$

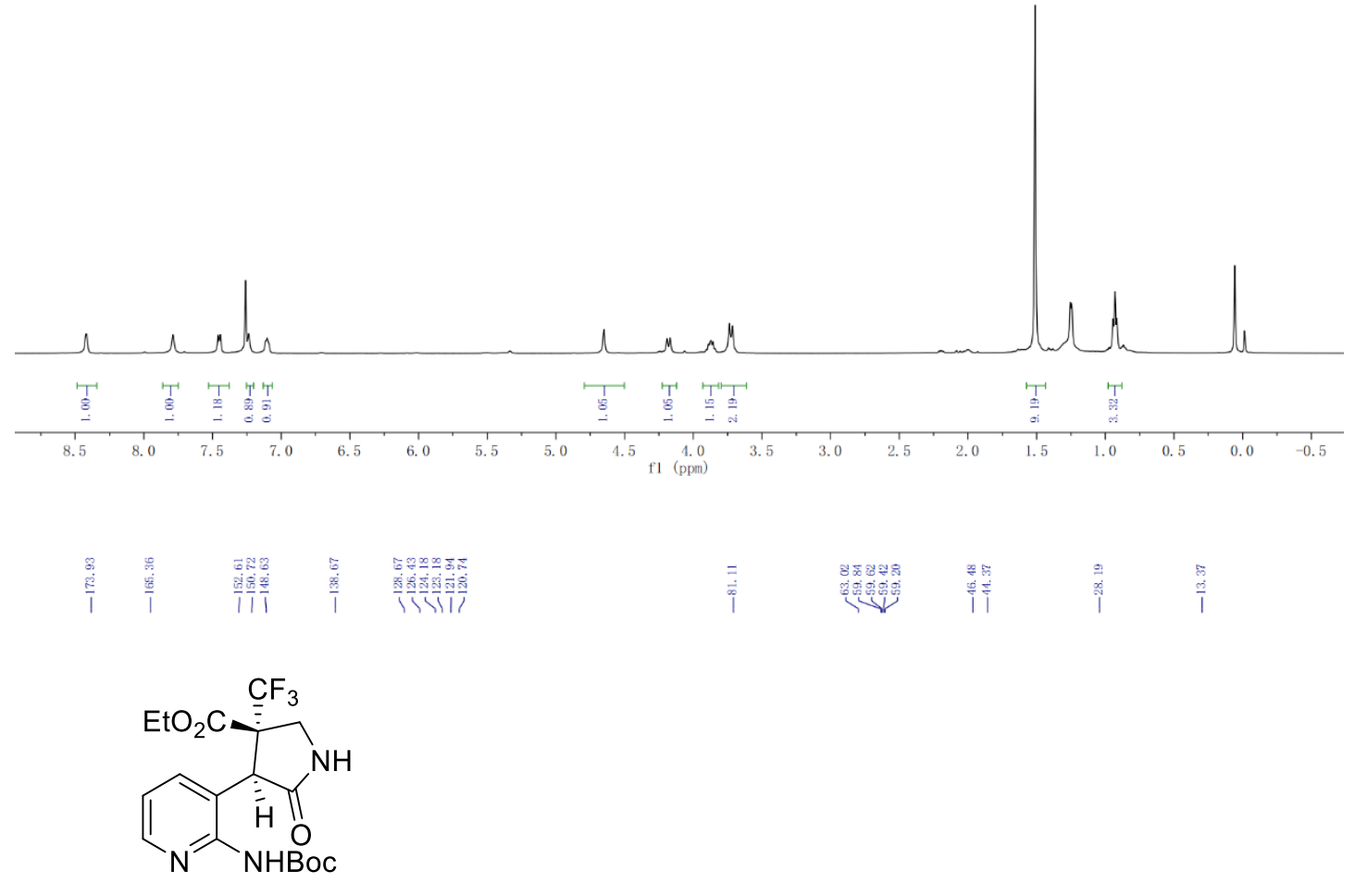

$3 n$

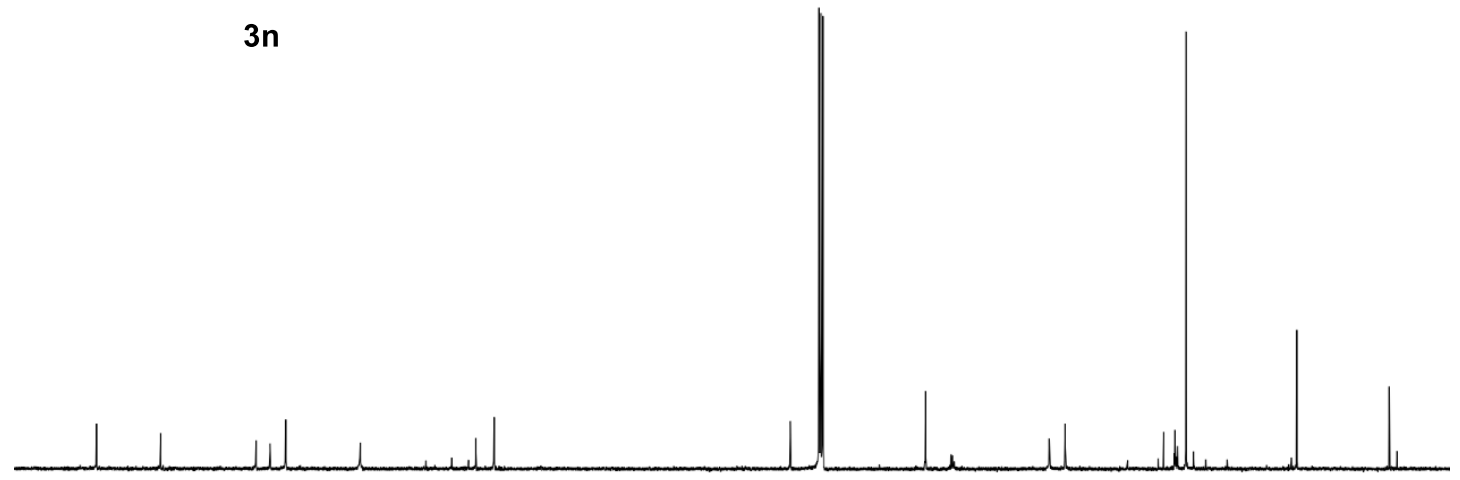

$180+170+160$

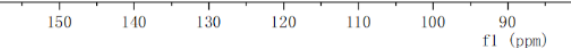




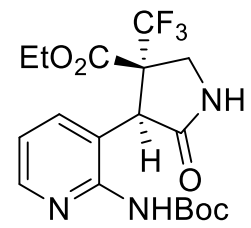

$3 n$

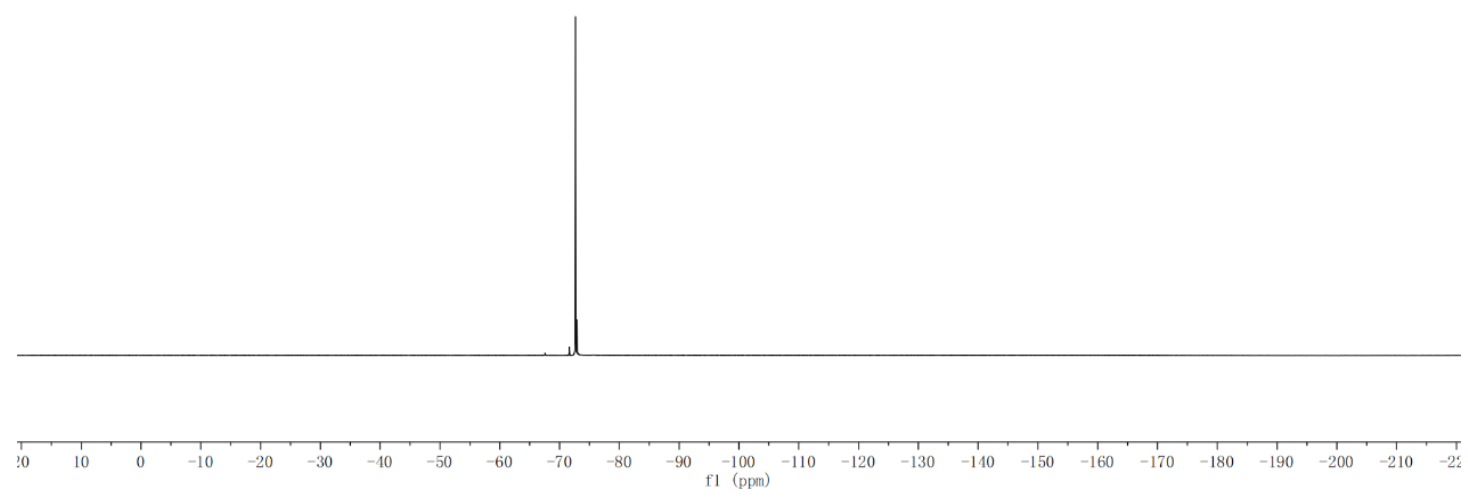

ethyl (3S,4S)-4-(2-acetamidophenyl)-5-oxo-3-(trifluoromethyl) pyrrolidine-3-carboxylate (3o)

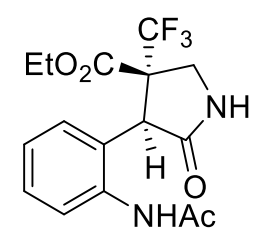

3o was obtained as a yellow oil in $82 \%$ yield $(5 \mathrm{~h}$ for Michael addition and 12 $\mathrm{h}$ for catalytic hydrogenation), $75 \%$ ee and $>20: 1 \mathrm{dr}$. The enantiomeric excess was determined by HPLC (Daicel Chiralpak AD-H, hexane/i-PrOH = 90:10 $(\mathrm{v} / \mathrm{v}), \lambda=254 \mathrm{~nm}$, flow rate $\left.=1.0 \mathrm{~mL} / \mathrm{min}, 25^{\circ} \mathrm{C}\right): t_{\text {minor }}=16.94 \mathrm{~min}, t_{\text {major }}=$ $15.94 \mathrm{~min} ;[\alpha]_{\mathrm{D}}{ }^{25} 13.21$ (c 0.37, MeOH); ${ }^{1} \mathrm{H}$ NMR $(500 \mathrm{MHz}$, Chloroform- $d$ ) $\delta$

30 $7.85(\mathrm{~s}, 1 \mathrm{H}), 7.62(\mathrm{~d}, J=8.2 \mathrm{~Hz}, 1 \mathrm{H}), 7.44(\mathrm{~s}, 1 \mathrm{H}), 7.31(\mathrm{t}, J=7.3 \mathrm{~Hz}, 1 \mathrm{H})$, $7.16(\mathrm{t}, J=7.6 \mathrm{~Hz}, 1 \mathrm{H}), 7.07(\mathrm{~d}, J=7.8 \mathrm{~Hz}, 1 \mathrm{H}), 4.38(\mathrm{~s}, 1 \mathrm{H}), 4.28(\mathrm{~d}, J=$ $11.7 \mathrm{~Hz}, 1 \mathrm{H}), 3.85(\mathrm{dq}, J=14.0,7.3 \mathrm{~Hz}, 1 \mathrm{H}), 3.79-3.68(\mathrm{~m}, 2 \mathrm{H}), 2.13(\mathrm{~s}, 3 \mathrm{H}), 0.87(\mathrm{t}, J=7.2 \mathrm{~Hz}$, 3H) ppm. ${ }^{13} \mathrm{C}$ NMR (125 MHz, Chloroform-d) $\delta 174.84,168.74,165.44,136.82,129.17,127.17$, 127.06, 126.06, 125.99, 125.58 (q, $J=283.7 \mathrm{~Hz}$ ), 63.27, 59.66 (q, $J=26.3 \mathrm{~Hz}), 44.18,29.68$, 23.81, 13.22 ppm; ${ }^{19} \mathrm{~F}$ NMR (471 MHz, Chloroform- $d$ ) $\delta-73.35$ ppm. ESI-HRMS m/z: 359.1211 $[\mathrm{M}+\mathrm{H}]{ }^{+}, \mathrm{C}_{16} \mathrm{H}_{17} \mathrm{~F}_{3} \mathrm{~N}_{2} \mathrm{O}_{4}+\mathrm{H}^{+}$requires 359.1213.
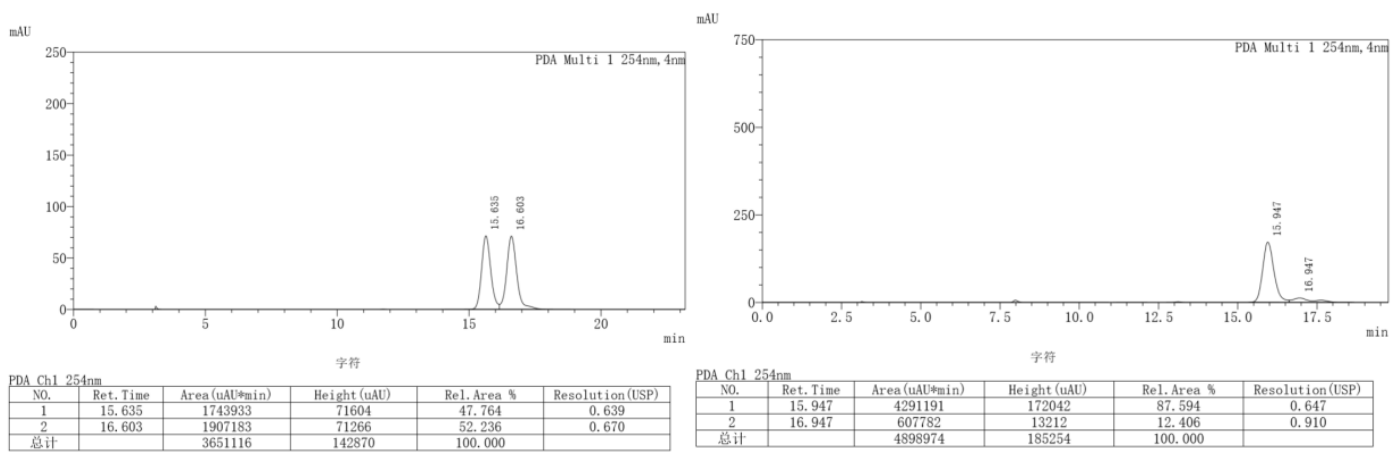


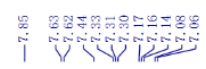

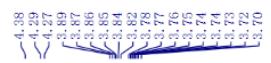

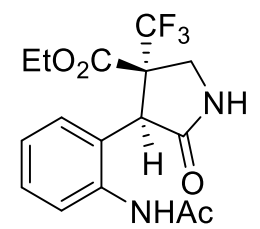

30

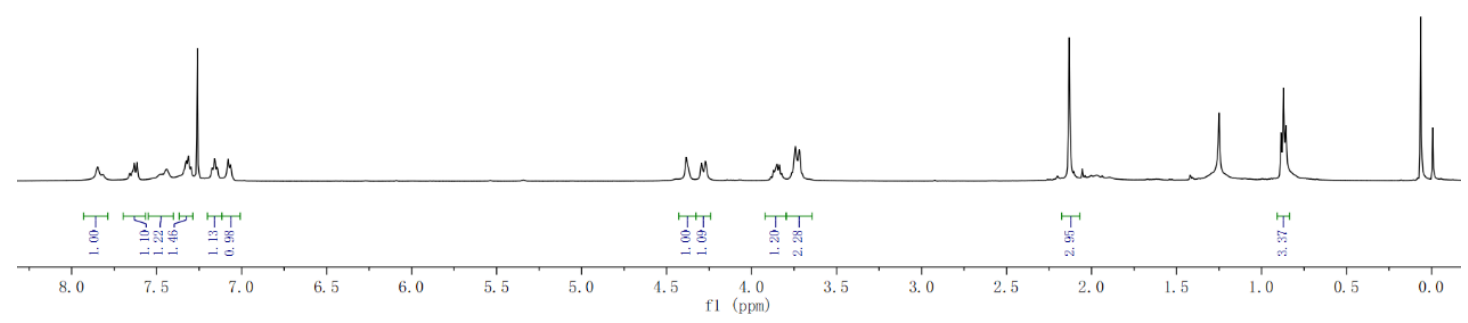

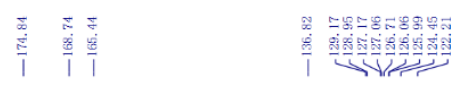

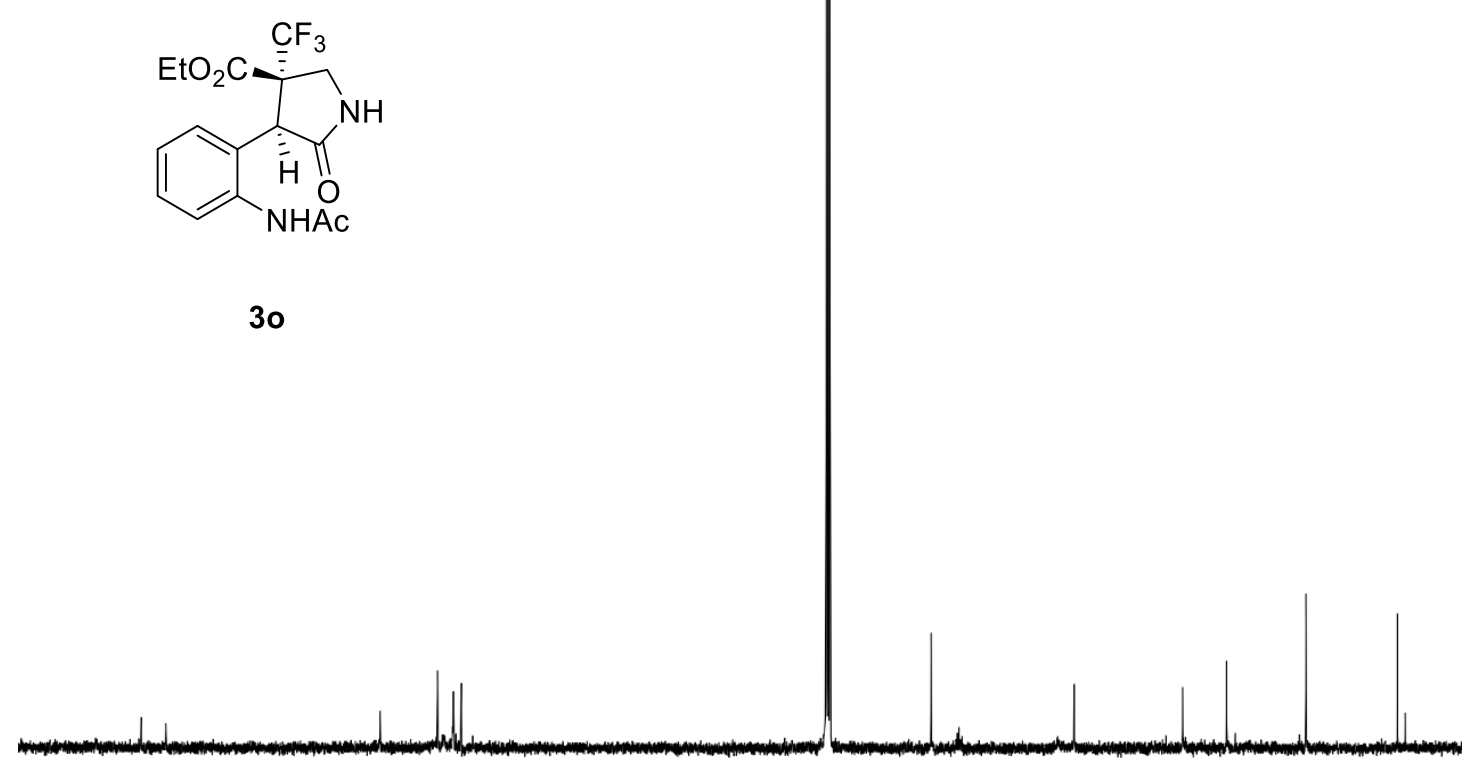

30

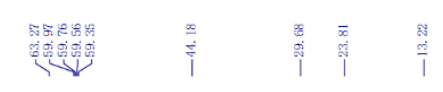

180

160

$150 \quad 140$

120

90
$\mathrm{fl}(\mathrm{ppm})$ 


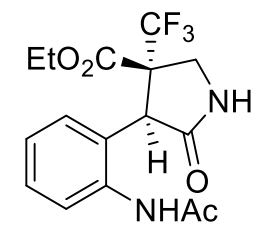

30

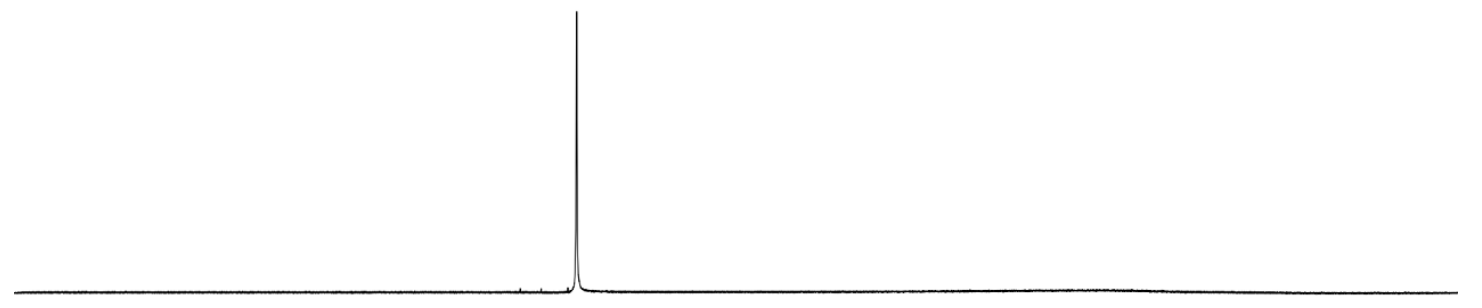

$\begin{array}{llllllllllllllllllllllllllllll}10 & 10 & 0 & -10 & -20 & -30 & -40 & -50 & -60 & -70 & -80 & -90 & -100 & -110 & -120 & -130 & -140 & -150 & -160 & -170 & -180 & -190 & -200 & -210 & -22\end{array}$

methyl

(3S,4S)-4-(2-((tert-butoxycarbonyl)amino)phenyl)-5-oxo-3-(trifluoromethyl)pyrrolidine-3-carbo xylate (3p)<smiles>COC(=O)C(F)(F)C1(C(F)(F)F)C2NC(=O)C1C2c1ccccc1NC(C)(C)C</smiles>

$3 p$

3p was obtained as a yellow foam in $90 \%$ yield (3 $\mathrm{h}$ for Michael addition and $10 \mathrm{~h}$ for catalytic hydrogenation), $95 \%$ ee and $>20: 1 \mathrm{dr}$. The enantiomeric excess was determined by HPLC (Daicel Chiralpak OD-H, hexane/i-PrOH $=85: 15(\mathrm{v} / \mathrm{v}), \lambda=254 \mathrm{~nm}$, flow rate $\left.=1.0 \mathrm{~mL} / \mathrm{min}, 25{ }^{\circ} \mathrm{C}\right): t_{\text {minor }}=9.50 \mathrm{~min}$, $t_{\text {major }}=11.01 \mathrm{~min} ;[\alpha]_{\mathrm{D}}{ }^{25} 38.74(c \quad 0.29, \mathrm{MeOH}) ;{ }^{1} \mathrm{H} \mathrm{NMR}(500 \mathrm{MHz}$, Chloroform- $d$ ) $\delta 7.61(\mathrm{~d}, J=8.4 \mathrm{~Hz}, 1 \mathrm{H}), 7.46(\mathrm{~s}, 1 \mathrm{H}), 7.30(\mathrm{t}, J=7.8 \mathrm{~Hz}$, $1 \mathrm{H}), 7.12(\mathrm{t}, J=7.4 \mathrm{~Hz}, 1 \mathrm{H}), 7.03(\mathrm{~d}, J=7.9 \mathrm{~Hz}, 1 \mathrm{H}), 6.73(\mathrm{~s}, 1 \mathrm{H}), 4.41-$ $4.16(\mathrm{~m}, 2 \mathrm{H}), 3.73(\mathrm{~d}, J=11.6 \mathrm{~Hz}, 1 \mathrm{H}), 3.32(\mathrm{~s}, 3 \mathrm{H}), 1.51(\mathrm{~s}, 9 \mathrm{H}) \mathrm{ppm} .{ }^{13} \mathrm{C}$ NMR (125 MHz, Chloroform- $d$ ) $\delta 164.70,152.48,152.33,136.13,128.11,125.83,124.94,124.48$ (q, $J=282.6 \mathrm{~Hz})$, 124.33, 124.18, 79.52, 62.08, 58.72 (q, $J=26.0 \mathrm{~Hz}), 52.32,43.16,27.27 \mathrm{ppm} ;{ }^{19} \mathrm{~F}$ NMR (471 $\mathrm{MHz}$, Chloroform- $d$ ) $\delta$-73.29 ppm. ESI-HRMS m/z: 403.1471 [M+ H] ${ }^{+}, \mathrm{C}_{18} \mathrm{H}_{21} \mathrm{~F}_{3} \mathrm{~N}_{2} \mathrm{O}_{5}+\mathrm{H}^{+}$ requires 403.1475 .
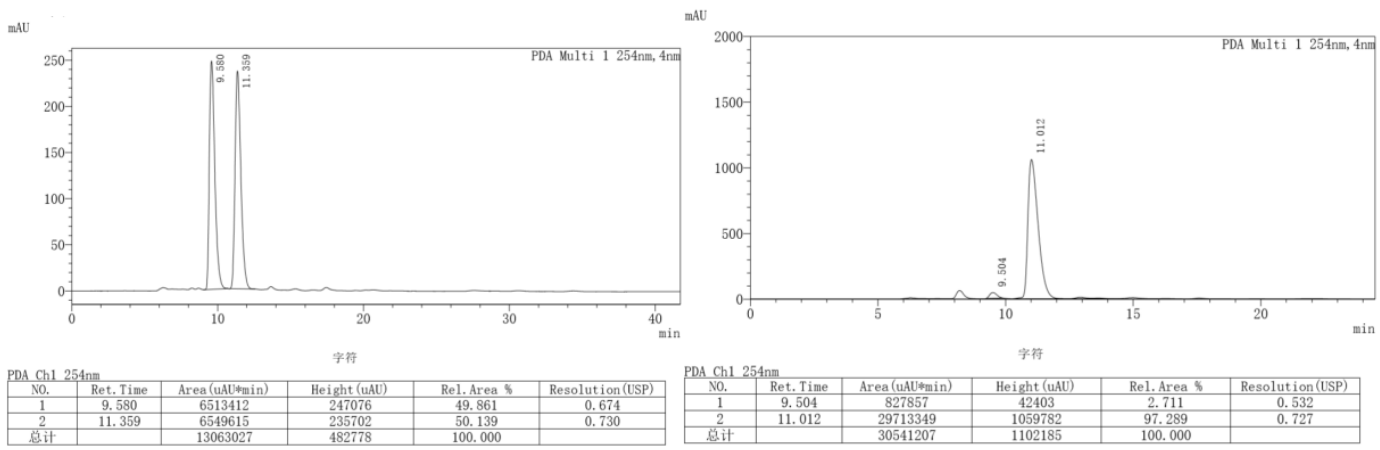


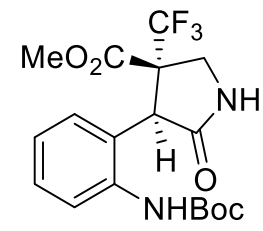

$3 p$

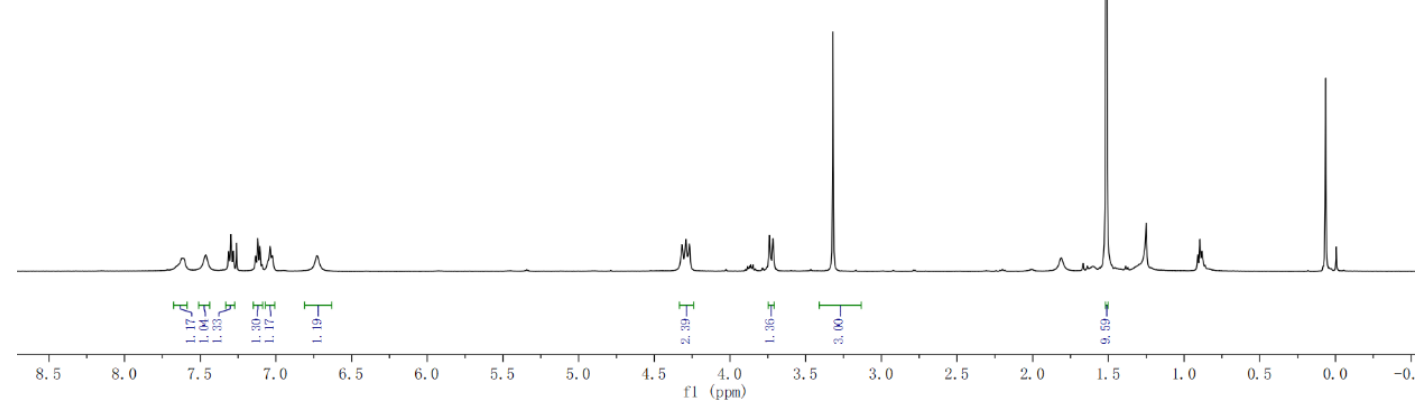

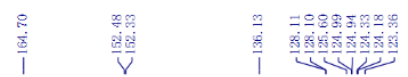

|<smiles>COC(=O)Nc1ccccc1[C@H]1C(=O)NC[C@@]1(C(C)(C)C)C(F)(F)F</smiles>

$3 p$

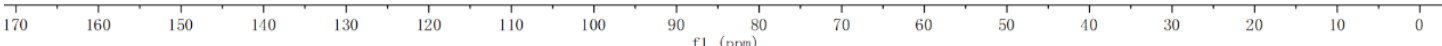




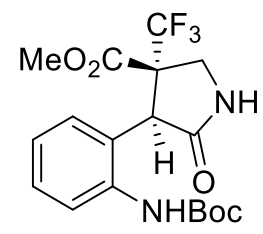

$3 p$

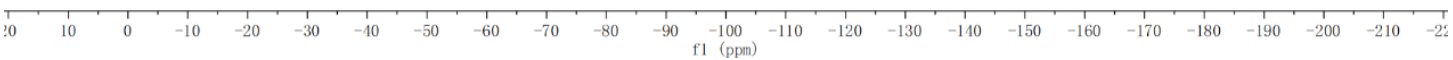

tert-butyl (2-((3R,4R)-2-oxo-4-(trifluoromethyl)pyrrolidin-3-yl)phenyl)carbamate (3q)<smiles>O=C1NC[C@H](c2ccccc2Nc2ccccc2)C1C(F)(F)F</smiles>

$3 q$

3q was obtained as a yellow oil in $85 \%$ yield ( $3 \mathrm{~h}$ for Michael addition and 11 $\mathrm{h}$ for catalytic hydrogenation), 95\% ee and > 20:1 dr. The enantiomeric excess was determined by HPLC (Daicel Chiralpak OD-H, hexane/i-PrOH =90:10 $(\mathrm{v} / \mathrm{v}), \lambda=254 \mathrm{~nm}$, flow rate $\left.=0.5 \mathrm{~mL} / \mathrm{min}, 25^{\circ} \mathrm{C}\right): t_{\text {minor }}=24.80 \mathrm{~min}, t_{\text {major }}=$ $14.43 \mathrm{~min} ;[\alpha]_{\mathrm{D}}{ }^{25} 44.21$ (c 0.65, MeOH); ${ }^{1} \mathrm{H}$ NMR (500 MHz, Chloroform- $d$ ) $\delta 7.72(\mathrm{~m}, 1 \mathrm{H}), 7.62(\mathrm{~s}, 1 \mathrm{H}), 7.39(\mathrm{~s}, 1 \mathrm{H}), 7.29(\mathrm{ddd}, J=8.3,6.8,2.1 \mathrm{~Hz}, 1 \mathrm{H})$, $7.22-6.96(\mathrm{~m}, 2 \mathrm{H}), 4.01(\mathrm{~d}, J=6.5 \mathrm{~Hz}, 1 \mathrm{H}), 3.65(\mathrm{dd}, J=10.7,8.8 \mathrm{~Hz}, 1 \mathrm{H}), 3.53(\mathrm{dd}, J=10.8$, $5.4 \mathrm{~Hz}, 1 \mathrm{H}), 3.44$ (tt, $J=8.6,6.0 \mathrm{~Hz}, 1 \mathrm{H}), 1.50$ (s, 9H) ppm. ${ }^{13} \mathrm{C}$ NMR (125 MHz, Chloroform- $d$ ) $\delta 176.45,154.12,136.95,129.83,129.36,128.59,126.54,126.44$ (q, $J=277.9 \mathrm{~Hz}), 125.46,80.49$, 43.86 (q, $J=28.4 \mathrm{~Hz}$ ), 40.49, 29.68, 28.30 ppm; ${ }^{19} \mathrm{~F}$ NMR (471 MHz, Chloroform- $d$ ) $\delta-71.96$ ppm. ESI-HRMS m/z: $345.1423[\mathrm{M}+\mathrm{H}]{ }^{+}, \mathrm{C}_{16} \mathrm{H}_{19} \mathrm{~F}_{3} \mathrm{~N}_{2} \mathrm{O}_{3}+\mathrm{H}^{+}$requires 345.1421.
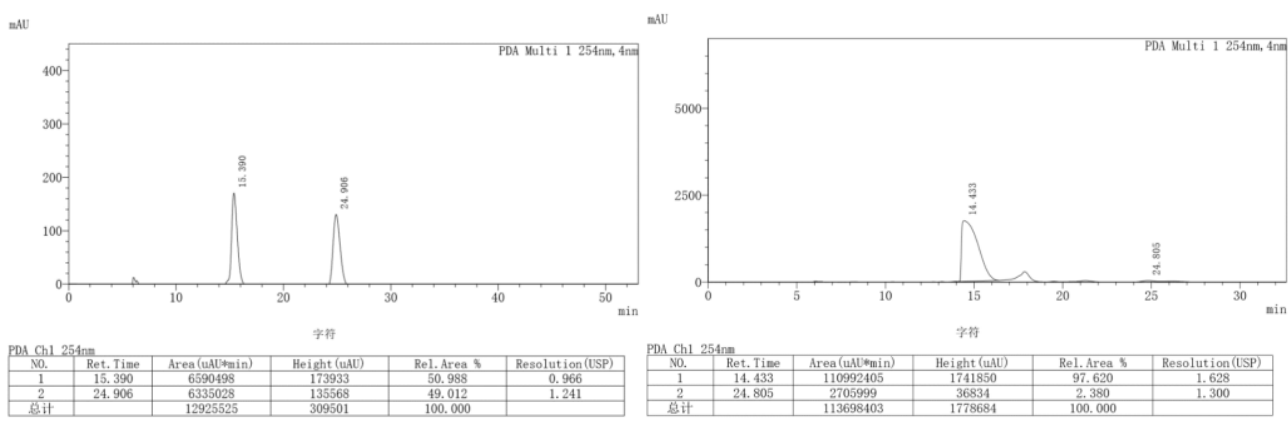

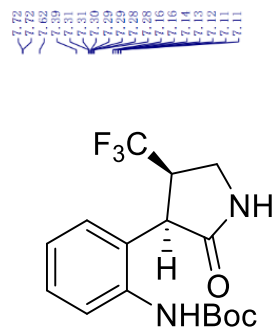

$3 q$
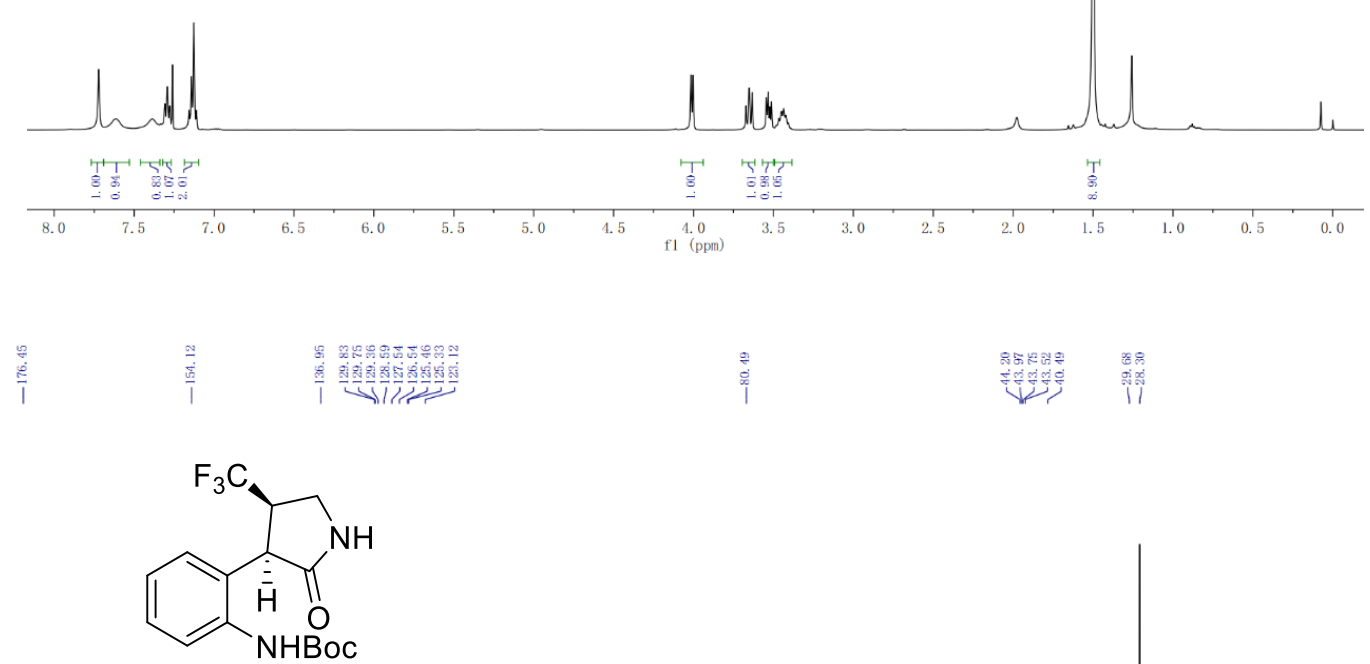

$3 q$

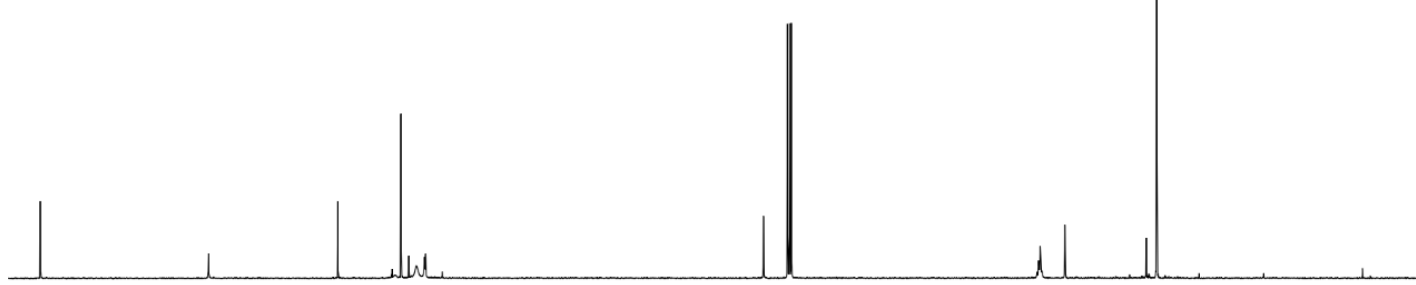

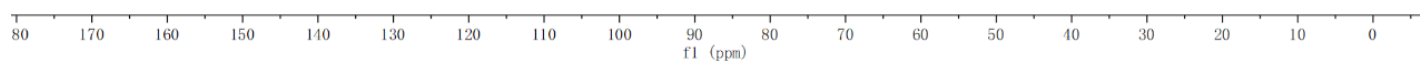




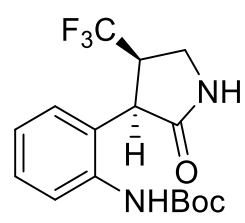

$3 q$

\section{$\frac{1}{20}$}

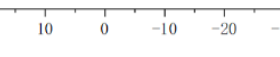

\section{ethyl}

(3R,4S)-4-(2-((tert-butoxycarbonyl)amino)phenyl)-3-methyl-5-oxopyrrolidine-3-carboxylate (3r)

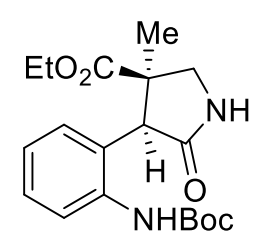

$3 \mathbf{r}$

3r was obtained as a yellow foam in $87 \%$ yield ( $4 \mathrm{~h}$ for Michael addition and $10 \mathrm{~h}$ for catalytic hydrogenation), $74 \%$ ee and $>20: 1 \mathrm{dr}$. The enantiomeric excess was determined by HPLC (Daicel Chiralpak AD-H, hexane/i-PrOH $=80: 20(\mathrm{v} / \mathrm{v}), \lambda=254 \mathrm{~nm}$, flow rate $\left.=1.0 \mathrm{~mL} / \mathrm{min}, 25^{\circ} \mathrm{C}\right): t_{\text {minor }}=4.50 \mathrm{~min}$, $t_{\text {major }}=6.07 \mathrm{~min} ;[\alpha]_{\mathrm{D}}{ }^{25} 15.73(c \quad 0.32, \mathrm{MeOH}) ;{ }^{1} \mathrm{H}$ NMR $(500 \mathrm{MHz}$, Chloroform- $d$ ) $\delta 7.66(\mathrm{~s}, 1 \mathrm{H}), 7.35(\mathrm{~s}, 1 \mathrm{H}), 7.25-7.08(\mathrm{~m}, 2 \mathrm{H}), 7.03(\mathrm{~m}, 2 \mathrm{H})$, $4.11(\mathrm{~d}, J=10.6 \mathrm{~Hz}, 1 \mathrm{H}), 3.88(\mathrm{q}, J=8.3,7.0 \mathrm{~Hz}, 1 \mathrm{H}), 3.86-3.76(\mathrm{~m}, 1 \mathrm{H})$, $3.72(\mathrm{~s}, 1 \mathrm{H}), 3.21(\mathrm{~d}, J=10.6 \mathrm{~Hz}, 1 \mathrm{H}), 1.63(\mathrm{~s}, 3 \mathrm{H}), 1.52(\mathrm{~s}, 9 \mathrm{H}), 0.91(\mathrm{t}, J=7.2 \mathrm{~Hz}, 3 \mathrm{H}) \mathrm{ppm} .{ }^{13} \mathrm{C}$ NMR (125 MHz, Chloroform- $d$ ) $\delta$ 176.90, 173.33, 153.55, 137.08, 129.90, 128.09, 126.63, 125.16, 124.68, 80.25, 61.37, 52.87, 50.61, 49.50, 28.39, 26.58, 13.54 ppm; ESI-HRMS m/z: 363.1913 [M $+\mathrm{H}]{ }^{+}, \mathrm{C}_{19} \mathrm{H}_{26} \mathrm{~N}_{2} \mathrm{O}_{5}+\mathrm{H}^{+}$requires 363.1914 .
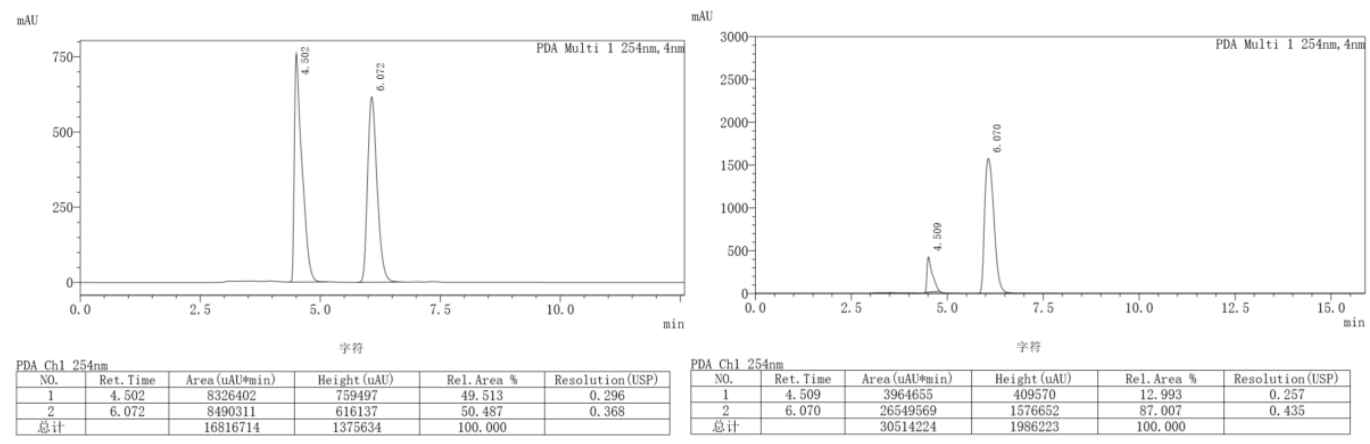


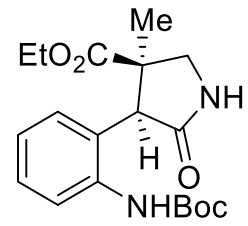

$3 r$

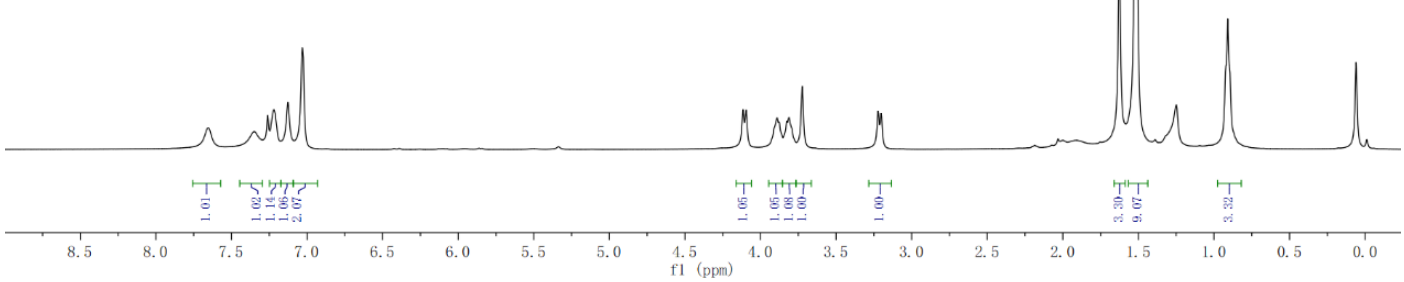

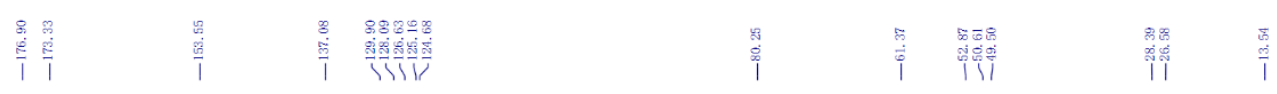<smiles>CCOC(=O)c1ccccc1NC(=O)C1C(=O)NCC1(C)C(C)(C)C</smiles>

$3 r$
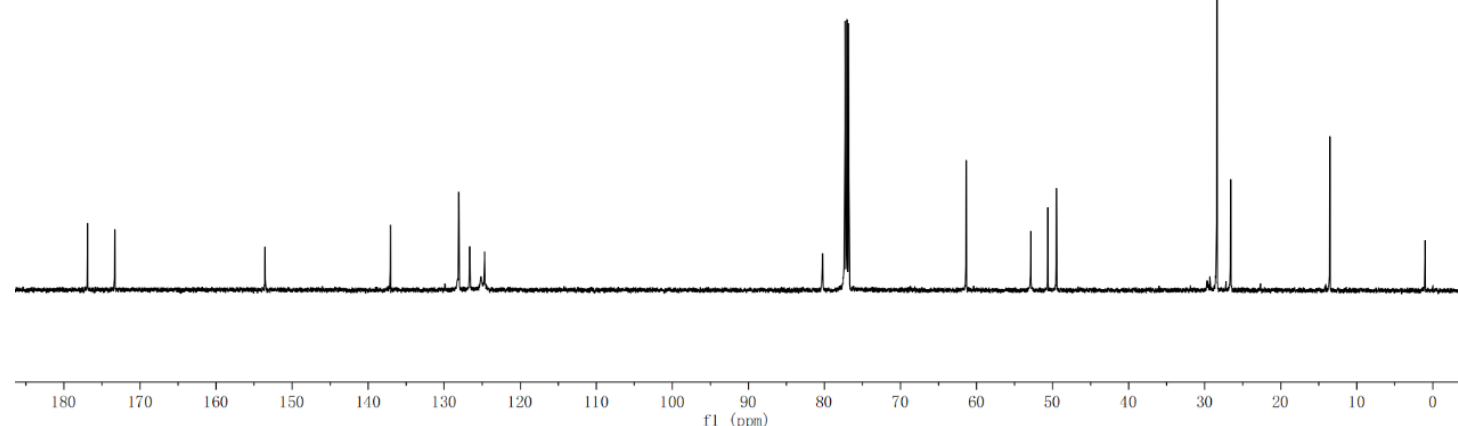

ethyl (3R,4R)-4-(2-((tert-butoxycarbonyl) amino) phenyl)-5-oxopyrrolidine-3-carboxylate (3s) 


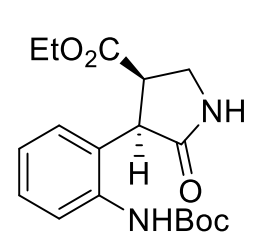

3s

3s was obtained as a yellow oil in $88 \%$ yield ( $4 \mathrm{~h}$ for Michael addition and $11 \mathrm{~h}$ for Catalytic hydrogenation), $51 \%$ ee and $>20: 1 \mathrm{dr}$. The enantiomeric excess was determined by HPLC (Daicel Chiralpak AD-H, hexane/i-PrOH $=95: 5$ $(\mathrm{v} / \mathrm{v}), \lambda=254 \mathrm{~nm}$, flow rate $\left.=0.5 \mathrm{~mL} / \mathrm{min}, 25^{\circ} \mathrm{C}\right): t_{\text {minor }}=79.29 \mathrm{~min}, t_{\text {major }}=$ $69.94 \mathrm{~min} ;[\alpha]_{\mathrm{D}}{ }^{25}-28.36$ ( $c$ $\left.0.17, \mathrm{MeOH}\right) ;{ }^{1} \mathrm{H}$ NMR $(500 \mathrm{MHz}$, Chloroform- $d$ ) $\delta 7.96(\mathrm{~s}, 1 \mathrm{H}), 7.73(\mathrm{~d}, J=8.1 \mathrm{~Hz}, 1 \mathrm{H}), 7.27(\mathrm{t}, J=7.5 \mathrm{~Hz}, 1 \mathrm{H}), 7.12(\mathrm{dt}, J=$ 14.8, 7.4 Hz, 2H), $6.92(\mathrm{~s}, 1 \mathrm{H}), 4.31-4.11(\mathrm{~m}, 3 \mathrm{H}), 3.72(\mathrm{t}, J=9.3 \mathrm{~Hz}, 1 \mathrm{H}), 3.62(\mathrm{dd}, J=9.9,6.9$ $\mathrm{Hz}, 1 \mathrm{H}), 3.53(\mathrm{q}, J=8.1 \mathrm{~Hz}, 1 \mathrm{H}), 1.52(\mathrm{~s}, 9 \mathrm{H}), 1.24(\mathrm{t}, J=7.2 \mathrm{~Hz}, 3 \mathrm{H}) \mathrm{ppm} .{ }^{13} \mathrm{C}$ NMR $(125 \mathrm{MHz}$, Chloroform- $d$ ) $\delta 176.90,172.28,153.76,137.34,128.69,128.17,128.10,126.88,124.61,80.15$, 61.89, 46.26, 45.75, 43.00, 28.39, 14.09 ppm; ESI-HRMS m/z: $349.1755[\mathrm{M}+\mathrm{H}]{ }^{+}$, $\mathrm{C}_{18} \mathrm{H}_{24} \mathrm{~N}_{2} \mathrm{O}_{5}+\mathrm{H}^{+}$requires 349.1758 .

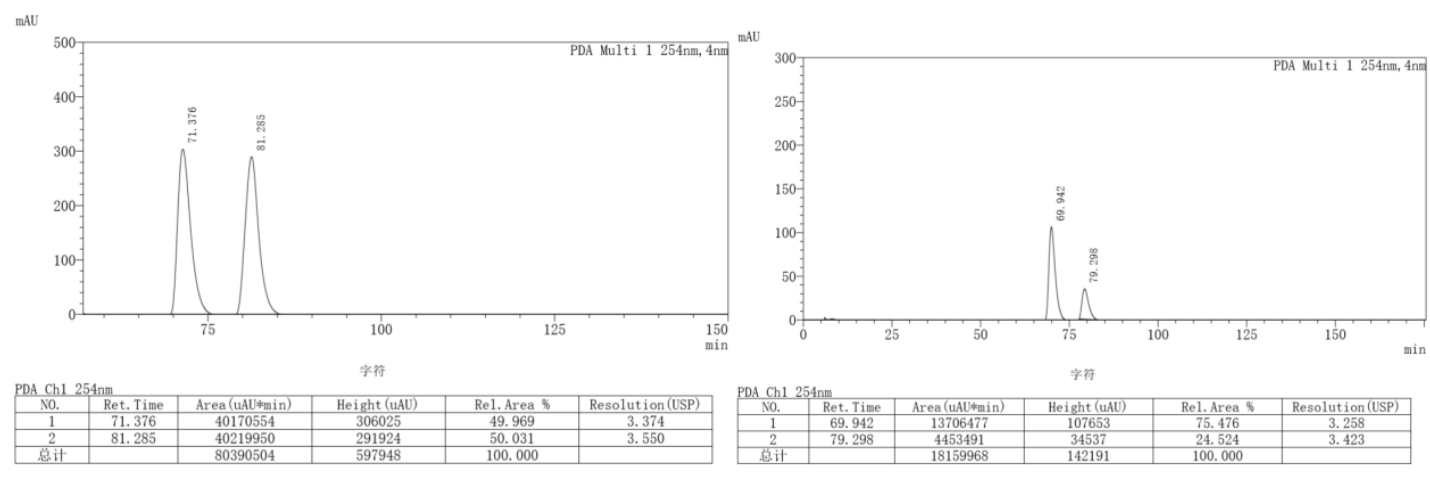

B
B
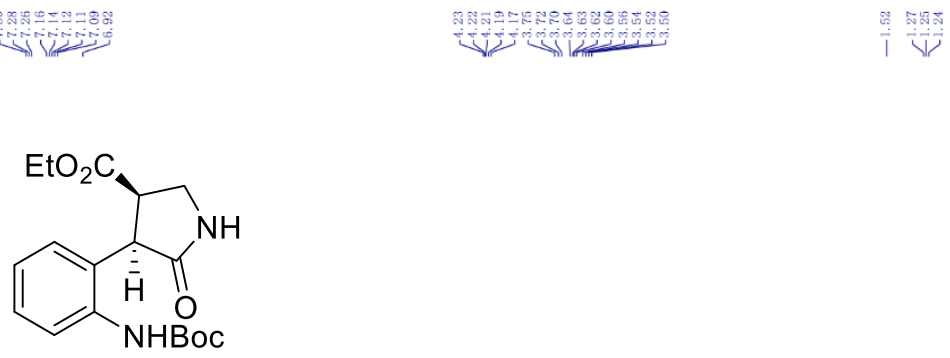

3s

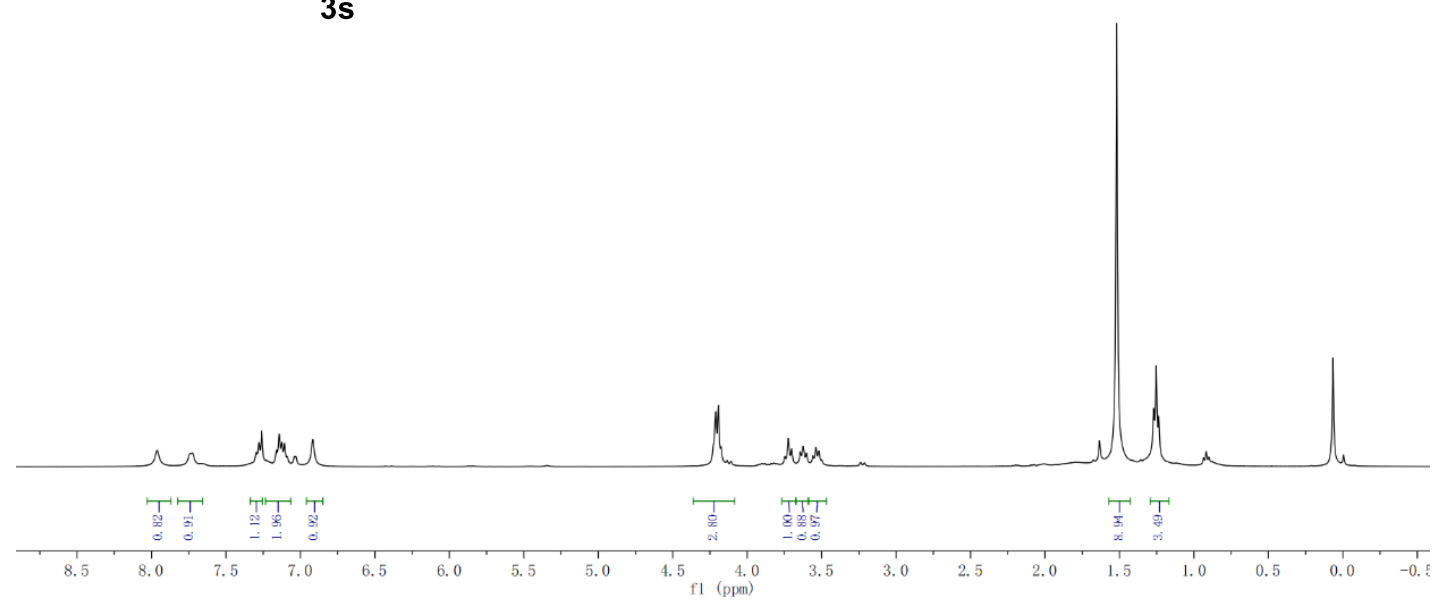




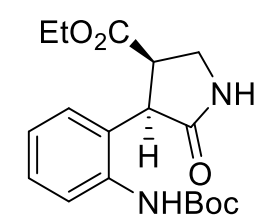

$3 \mathbf{s}$

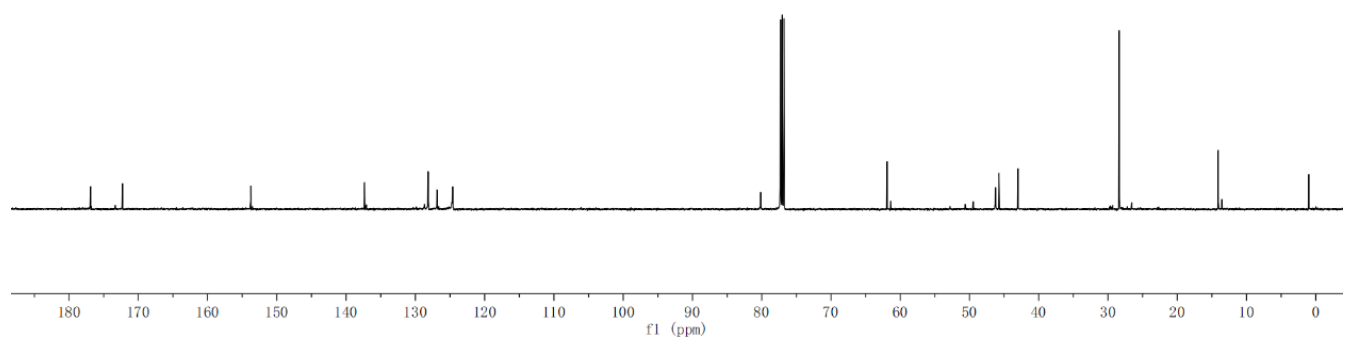

ethyl

(2S,3S,4S)-4-(2-((tert-butoxycarbonyl)amino)phenyl)-2-methyl-5-oxo-3-(trifluoromethyl)pyrroli dine-3-carboxylate (3t-major)<smiles>CCOC(=O)[C@@]1(c2ccccc2N)C(=O)N[C@@H](C)[C@H]1C(F)(F)F</smiles>

3t-major

3t-major was obtained as a yellow oil in $68 \%$ yield $(6 \mathrm{~h}$ for Michael addition and $18 \mathrm{~h}$ for catalytic hydrogenation), 95\% ee. The enantiomeric excess was determined by HPLC (Daicel Chiralpak AD-H, hexane/i-PrOH $=90: 10(\mathrm{v} / \mathrm{v}), \lambda=254 \mathrm{~nm}$, flow rate $\left.=0.5 \mathrm{~mL} / \mathrm{min}, 25^{\circ} \mathrm{C}\right): t_{\text {minor }}=33.18 \mathrm{~min}$, $t_{\text {major }}=35.29 \mathrm{~min} ;[\alpha]_{\mathrm{D}}{ }^{25} 38.37(c 0.44, \mathrm{MeOH}) ;{ }^{1} \mathrm{H}$ NMR $(500 \mathrm{MHz}$, Chloroform- $d$ ) $\delta 7.78(\mathrm{~d}, \mathrm{~J}=9.6 \mathrm{~Hz}, 1 \mathrm{H}), 7.71(\mathrm{~s}, 1 \mathrm{H}), 7.29(\mathrm{dt}, \mathrm{J}=8.5,4.4$ $4.36(\mathrm{~s}, 1 \mathrm{H}), 3.93(\mathrm{dq}, \mathrm{J}=14.2,7.2 \mathrm{~Hz}, 1 \mathrm{H}), 3.79(\mathrm{dq}, \mathrm{J}=10.7,7.1 \mathrm{~Hz}, 1 \mathrm{H}), 1.51(\mathrm{~s}, 9 \mathrm{H}), 1.44$ (d, $\mathrm{J}=6.8 \mathrm{~Hz}, 3 \mathrm{H}), 0.93(\mathrm{t}, \mathrm{J}=7.1 \mathrm{~Hz}, 3 \mathrm{H}) \mathrm{ppm} .{ }^{13} \mathrm{C}$ NMR $(125 \mathrm{MHz}$, Chloroform- $d$ ) $\delta 173.72$, $165.15,153.24,137.49,129.12,126.80,125.63,125.23$ (q, $J=285.2 \mathrm{~Hz}), 125.17,125.00,80.51$, 62.99, 62.64 (q, $J=23.3 \mathrm{~Hz}), 52.12,47.63,28.31,14.87,13.22 \mathrm{ppm} ;{ }^{19} \mathrm{~F}$ NMR $(471 \mathrm{MHz}$, Chloroform- $d$ ) $\delta$-66.74 ppm. ESI-HRMS m/z: $431.1786[\mathrm{M}+\mathrm{H}]{ }^{+}, \mathrm{C}_{20} \mathrm{H}_{25} \mathrm{~F}_{3} \mathrm{~N}_{2} \mathrm{O}_{5}+\mathrm{H}^{+}$requires 431.1788 .
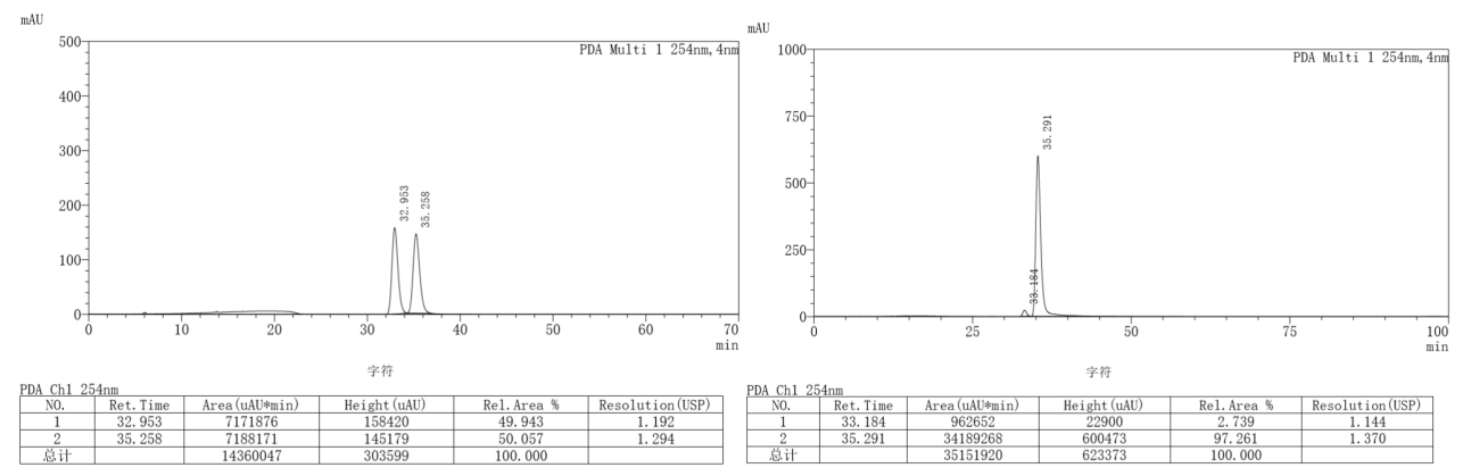
<smiles>CCOC(=O)[C@@]1(C(F)(F)F)[C@H](c2ccccc2N)C(=O)N[C@@H]1C</smiles>

3t-major

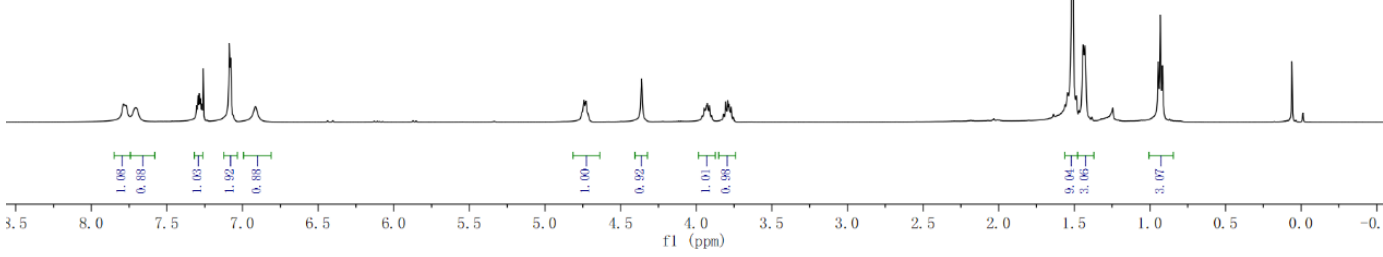

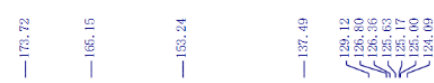
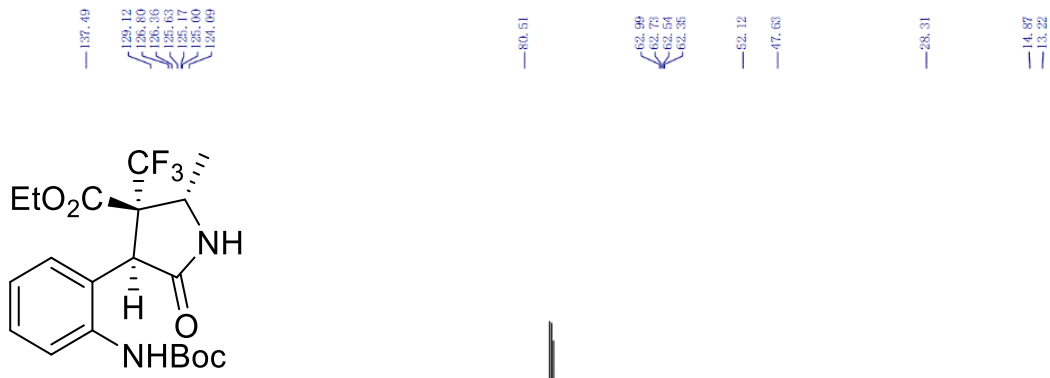

3t-major

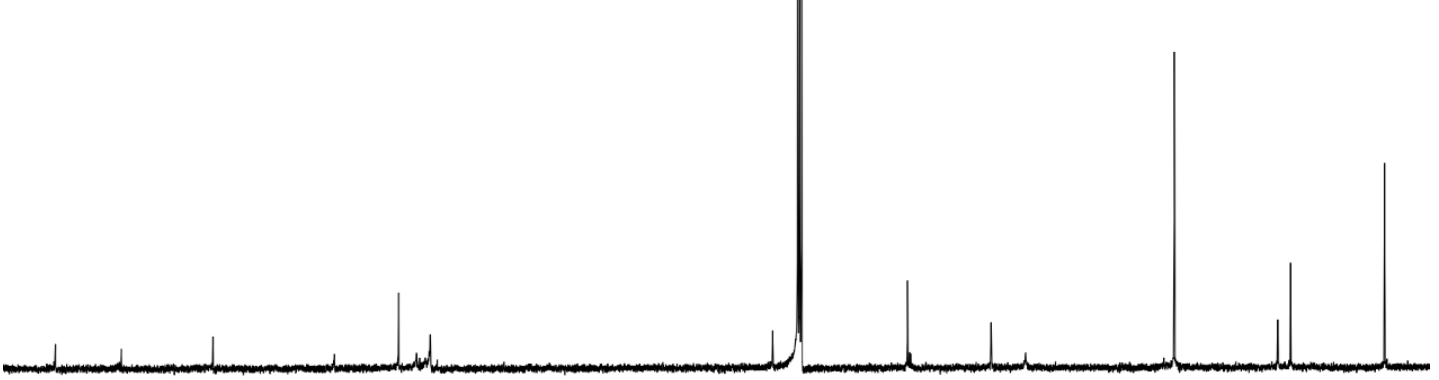

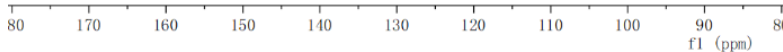


<smiles>CCOC(=O)c1ccccc1Nc1ccccc1C1C(=O)N[C@H](C)C1(C(F)(F)F)C(F)(F)F</smiles>

3t-major

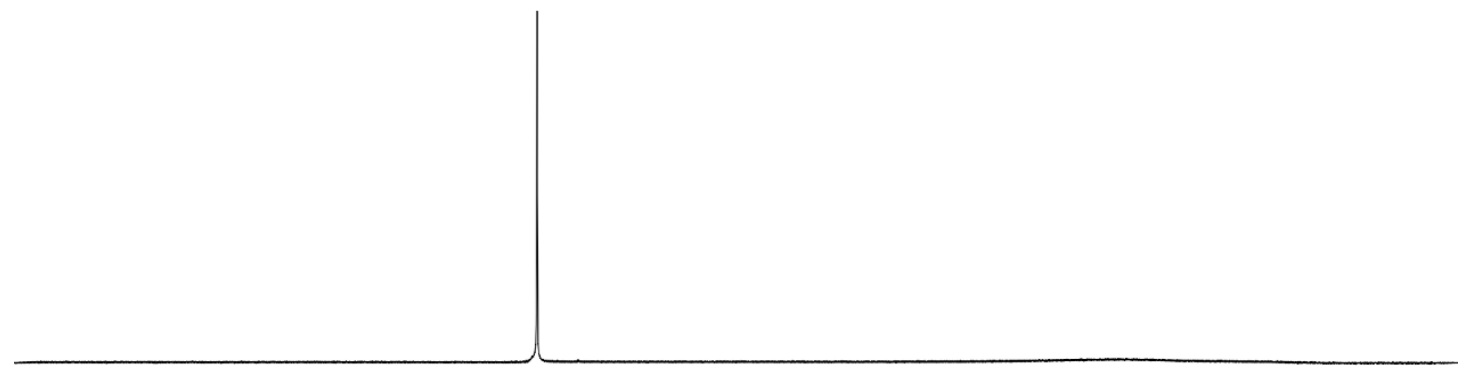

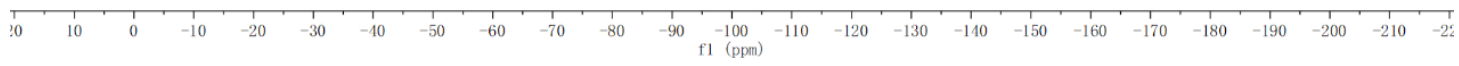

ethyl

(2R,3S,4S)-4-(2-((tert-butoxycarbonyl)amino)phenyl)-2-methyl-5-oxo-3-(trifluoromethyl)pyrroli dine-3-carboxylate (3t-minor)<smiles>CCOC(=O)c1ccccc1C1C(=O)NC(C)[C@]1(C(C)(F)F)C(F)(F)F</smiles>

3t-minor

3t-minor was obtained as a yellow oil in $15 \%$ yield $(6 \mathrm{~h}$ for Michael addition and $18 \mathrm{~h}$ for catalytic hydrogenation), $95 \%$ ee. The enantiomeric excess was determined by HPLC (Daicel Chiralpak AD-H, hexane/i-PrOH $=80: 20(\mathrm{v} / \mathrm{v}), \lambda=254 \mathrm{~nm}$, flow rate $\left.=1.0 \mathrm{~mL} / \mathrm{min}, 25^{\circ} \mathrm{C}\right): t_{\text {minor }}=5.42 \mathrm{~min}$, $t_{\text {major }}=7.21 \mathrm{~min} ;[\alpha]_{\mathrm{D}}{ }^{25} 15.17(c 0.11, \mathrm{MeOH}) ;{ }^{1} \mathrm{H} \mathrm{NMR}(500 \mathrm{MHz}$, Chloroform- $d$ ) $\delta 7.75(\mathrm{~s}, 2 \mathrm{H}), 7.30(\mathrm{t}, J=7.9 \mathrm{~Hz}, 1 \mathrm{H}), 7.23(\mathrm{~d}, J=7.7 \mathrm{~Hz}$, $1 \mathrm{H}), 7.10(\mathrm{t}, J=7.6 \mathrm{~Hz}, 1 \mathrm{H}), 6.92(\mathrm{~m}, 1 \mathrm{H}), 4.68(\mathrm{~s}, 1 \mathrm{H}), 4.40(\mathrm{q}, J=7.1 \mathrm{~Hz}$, 2H), $4.34(\mathrm{q}, J=6.9 \mathrm{~Hz}, 1 \mathrm{H}), 1.51(\mathrm{~s}, 9 \mathrm{H}), 1.36(\mathrm{t}, J=7.1 \mathrm{~Hz}, 3 \mathrm{H}), 1.25(\mathrm{~d}, J=6.9 \mathrm{~Hz}, 3 \mathrm{H}) \mathrm{ppm}$. ${ }^{13} \mathrm{C}$ NMR (125 MHz, Chloroform- $d$ ) $\delta$ 173.04, 168.90, 153.48, 138.10, 131.89, 131.86, 129.88, $128.82,125.98$ (q, $J=248.8 \mathrm{~Hz}), 123.78,80.23,64.03,63.39$ (q, $J=24.1 \mathrm{~Hz}), 53.01,49.21,28.36$, 16.96, 13.88 ppm; ${ }^{19} \mathrm{~F}$ NMR (471 MHz, Chloroform- $d$ ) $\delta-67.95$ ppm. ESI-HRMS m/z: 431.1786 $[\mathrm{M}+\mathrm{H}]^{+}, \mathrm{C}_{20} \mathrm{H}_{25} \mathrm{~F}_{3} \mathrm{~N}_{2} \mathrm{O}_{5}+\mathrm{H}^{+}$requires 431.1788 .
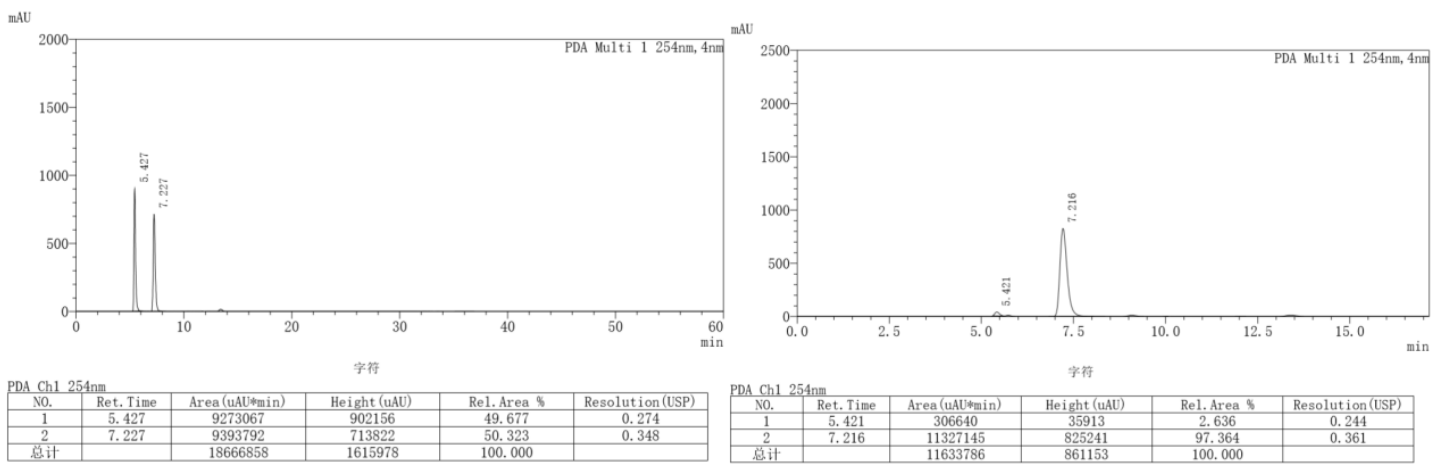


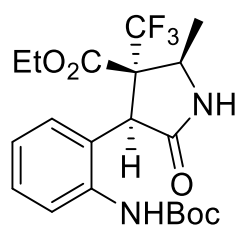

3t-minor
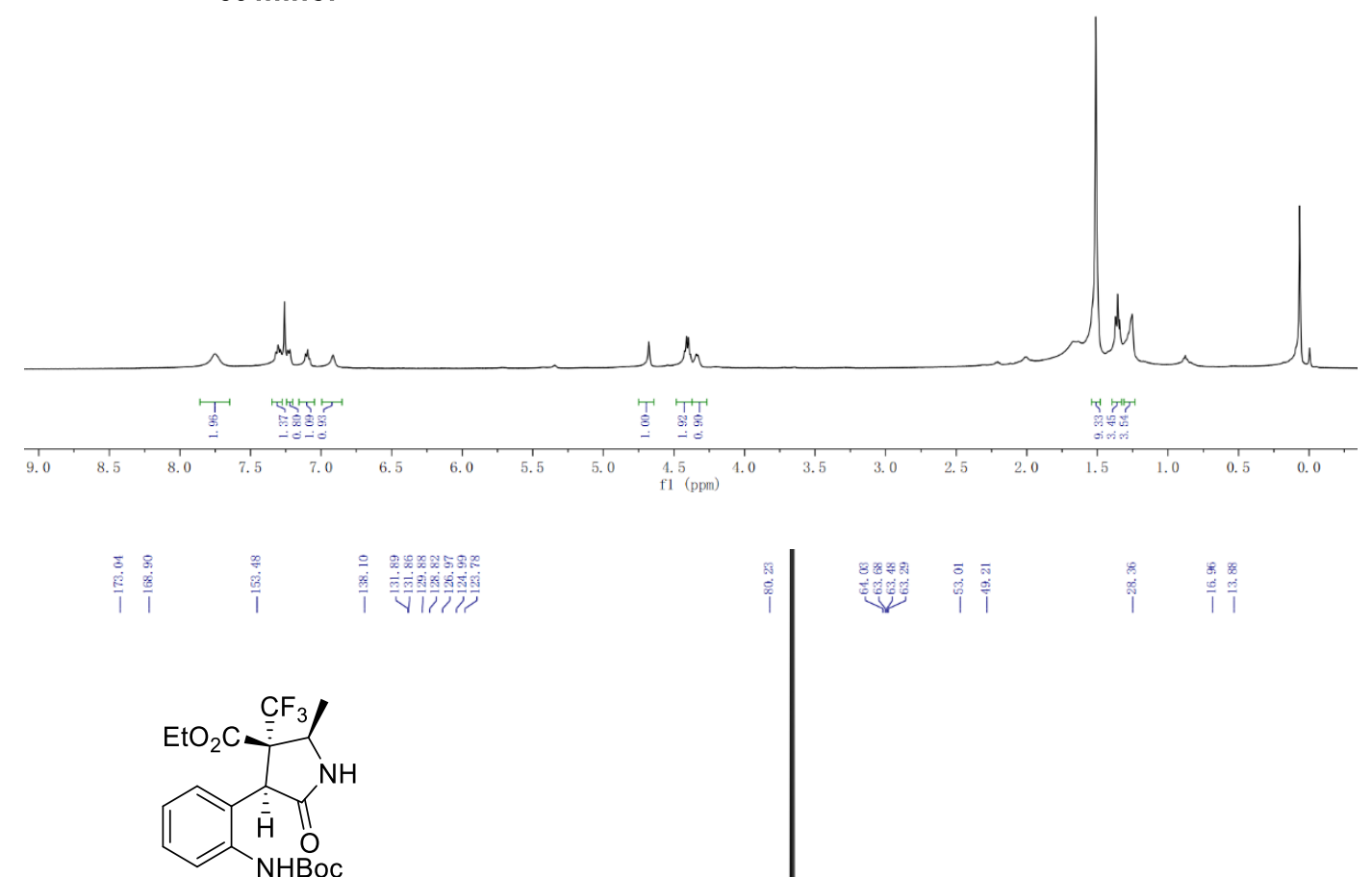

을

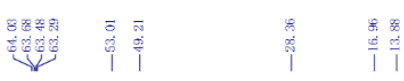

3t-minor

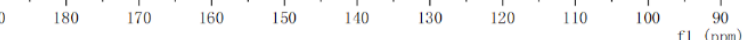




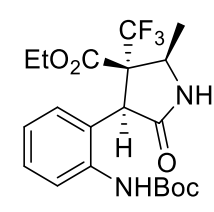

3t-minor

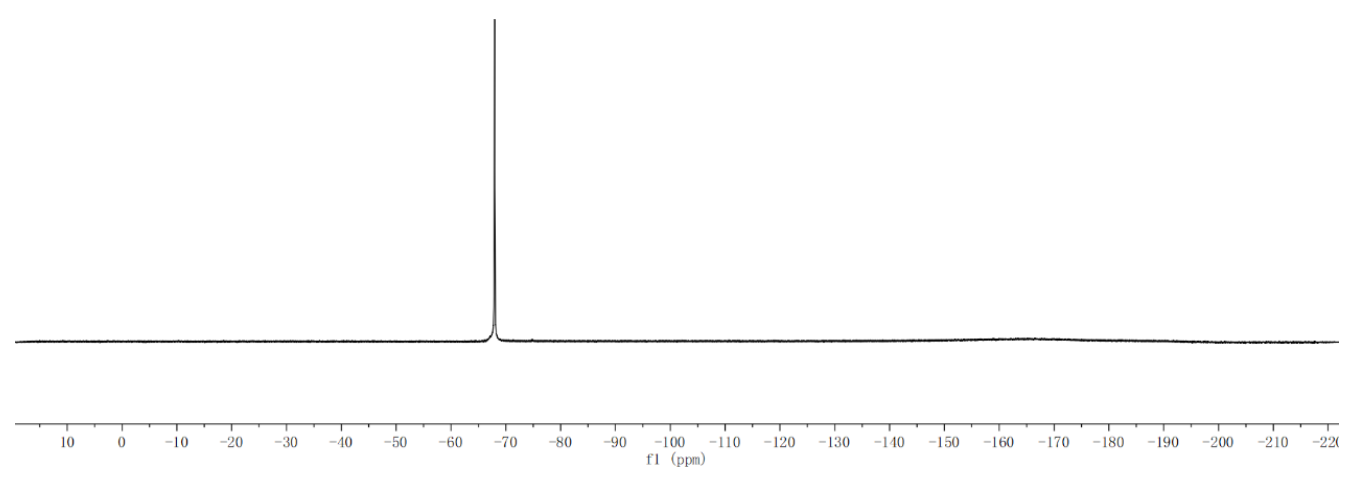

ethyl

(2S,3S,4S)-4-(2-((tert-butoxycarbonyl)amino)phenyl)-2-ethyl-5-oxo-3-(trifluoromethyl)pyrrolidi ne-3-carboxylate (3u-major)

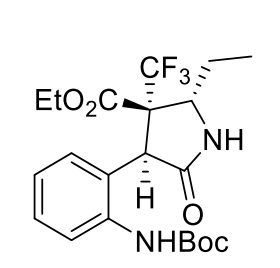

3u-major

3u-major was obtained as a yellow oil in $41 \%$ yield $(6 \mathrm{~h}$ for Michael addition and $30 \mathrm{~h}$ for catalytic hydrogenation), $90 \%$ ee. The enantiomeric excess was determined by HPLC (Daicel Chiralpak AD-H, hexane/i-PrOH $=80: 20(\mathrm{v} / \mathrm{v}), \lambda$ $=254 \mathrm{~nm}$, flow rate $\left.=1.0 \mathrm{~mL} / \mathrm{min}, 25^{\circ} \mathrm{C}\right): t_{\text {minor }}=5.82 \mathrm{~min}, t_{\text {major }}=4.16 \mathrm{~min}$; $[\alpha]_{\mathrm{D}}^{25} 68.73\left(c\right.$ 0.50, MeOH); ${ }^{1} \mathrm{H}$ NMR (500 MHz, Chloroform- $d$ ) $\delta 8.30$ (s, $1 \mathrm{H}), 7.88(\mathrm{~d}, J=8.2 \mathrm{~Hz}, 1 \mathrm{H}), 7.45(\mathrm{ddd}, J=10.1,5.2,2.2 \mathrm{~Hz}, 1 \mathrm{H}), 7.25(\mathrm{~d}, J$ $=5.7 \mathrm{~Hz}, 2 \mathrm{H}), 7.14(\mathrm{~s}, 1 \mathrm{H}), 4.65(\mathrm{~d}, J=10.3 \mathrm{~Hz}, 1 \mathrm{H}), 4.52(\mathrm{~s}, 1 \mathrm{H}), 4.08(\mathrm{dq}, J=14.3,7.0 \mathrm{~Hz}, 1 \mathrm{H})$, $3.95(\mathrm{dq}, J=10.9,7.2 \mathrm{~Hz}, 1 \mathrm{H}), 2.12-2.00(\mathrm{~m}, 1 \mathrm{H}), 1.91-1.78(\mathrm{~m}, 1 \mathrm{H}), 1.68(\mathrm{~s}, 9 \mathrm{H}), 1.24(\mathrm{t}, J=$ $7.4 \mathrm{~Hz}, 3 \mathrm{H}), 1.10$ (t, $J=5.8 \mathrm{~Hz}, 3 \mathrm{H}) \mathrm{ppm} .{ }^{13} \mathrm{C}$ NMR $(125 \mathrm{MHz}$, Chloroform- $d$ ) $\delta 174.37,165.26$, 153.27, 137.44, 129.04, 127.97, 126.91, 125.63, 125.31 (q, $J=286.2 \mathrm{~Hz}), 124.88,80.44,62.90$, $62.47(\mathrm{q}, J=23.4 \mathrm{~Hz}), 58.91,47.87,28.30,22.73,13.19,12.37 \mathrm{ppm} ;{ }^{19} \mathrm{~F}$ NMR $(471 \mathrm{MHz}$, Chloroform- $d$ ) $\delta-66.42$ ppm. ESI-HRMS m/z: $445.1944[\mathrm{M}+\mathrm{H}]{ }^{+}, \mathrm{C}_{21} \mathrm{H}_{27} \mathrm{~F}_{3} \mathrm{~N}_{2} \mathrm{O}_{5}+\mathrm{H}^{+}$requires 445.1945 .
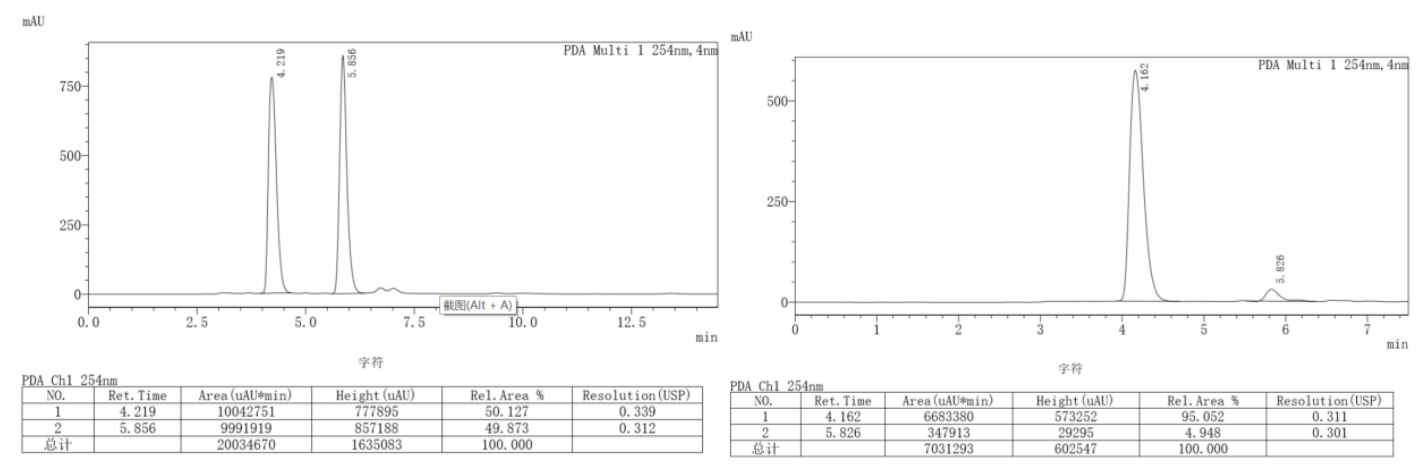


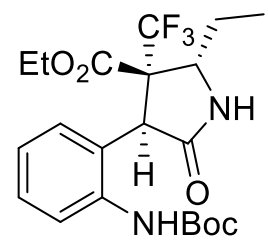

3u-major
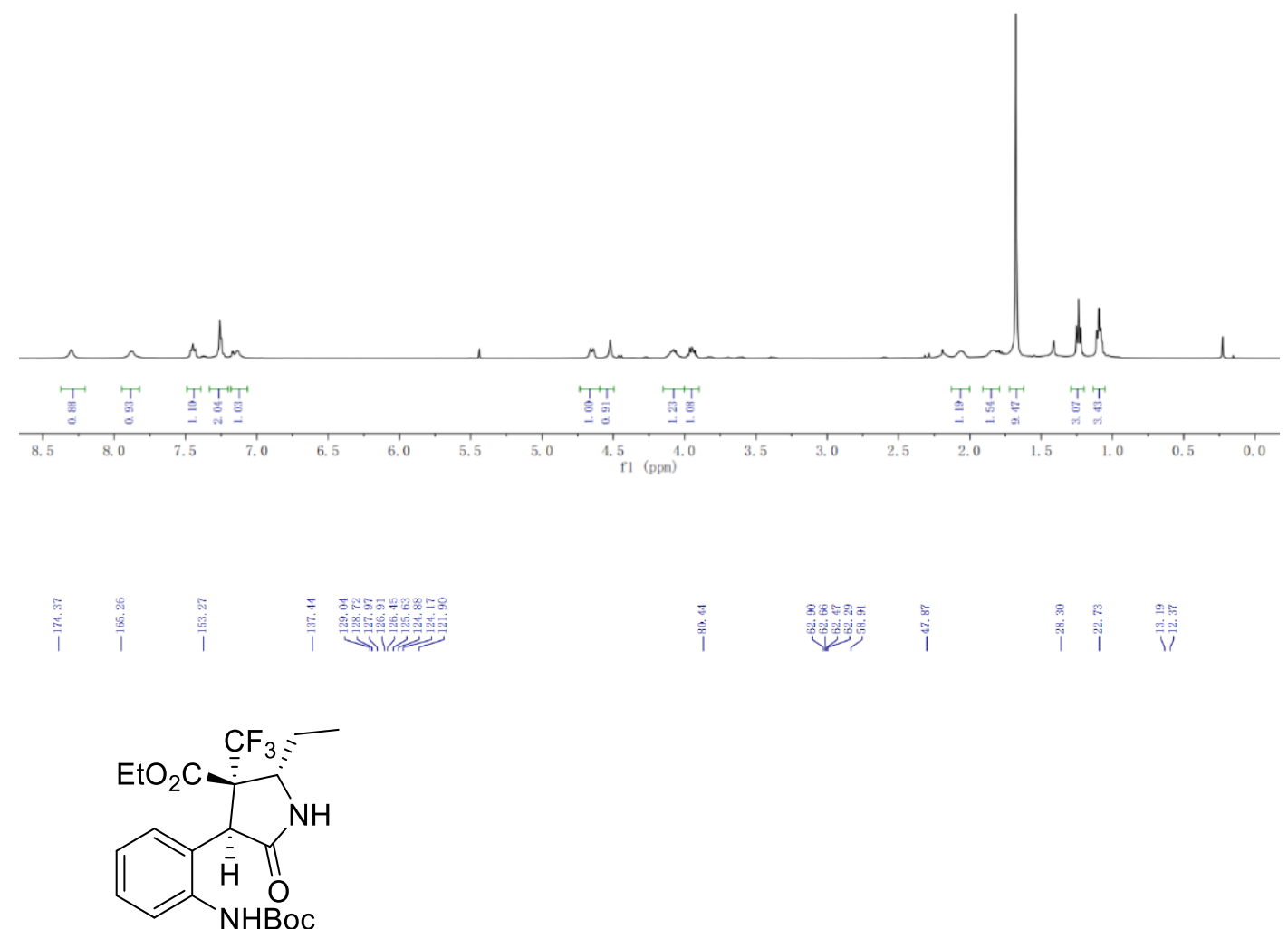

3u-major

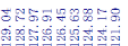

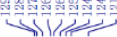

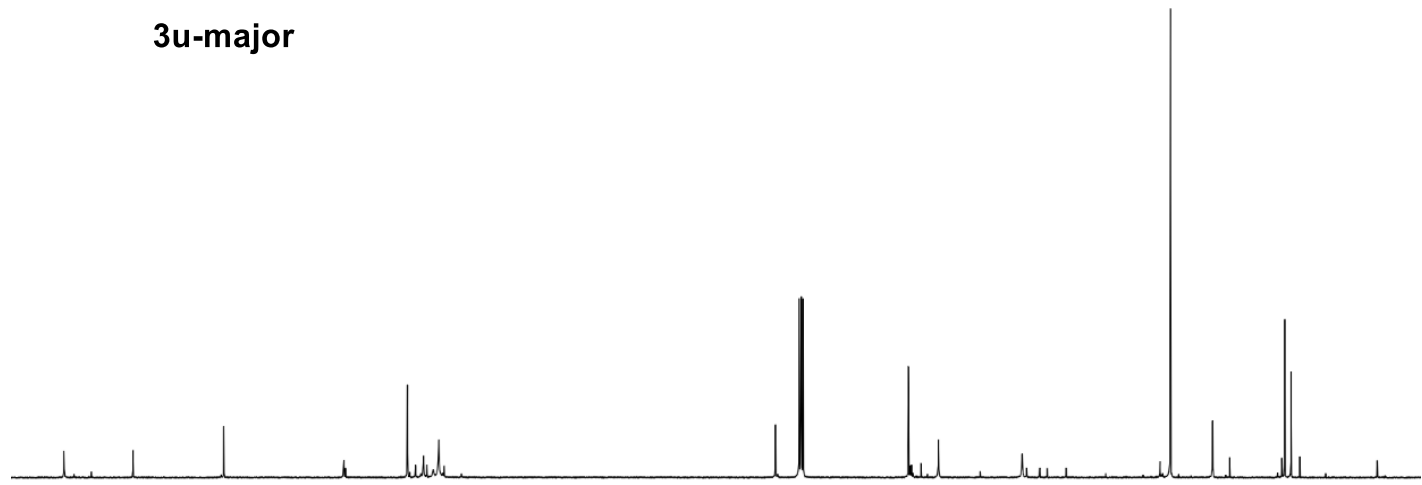

$\stackrel{T}{180}$

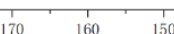

${ }_{140}^{1}+130$

$120 \quad 110 \quad 100$

f1 90 


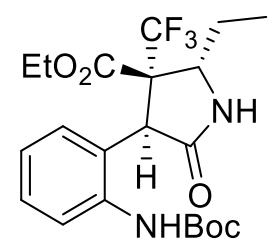

3u-major

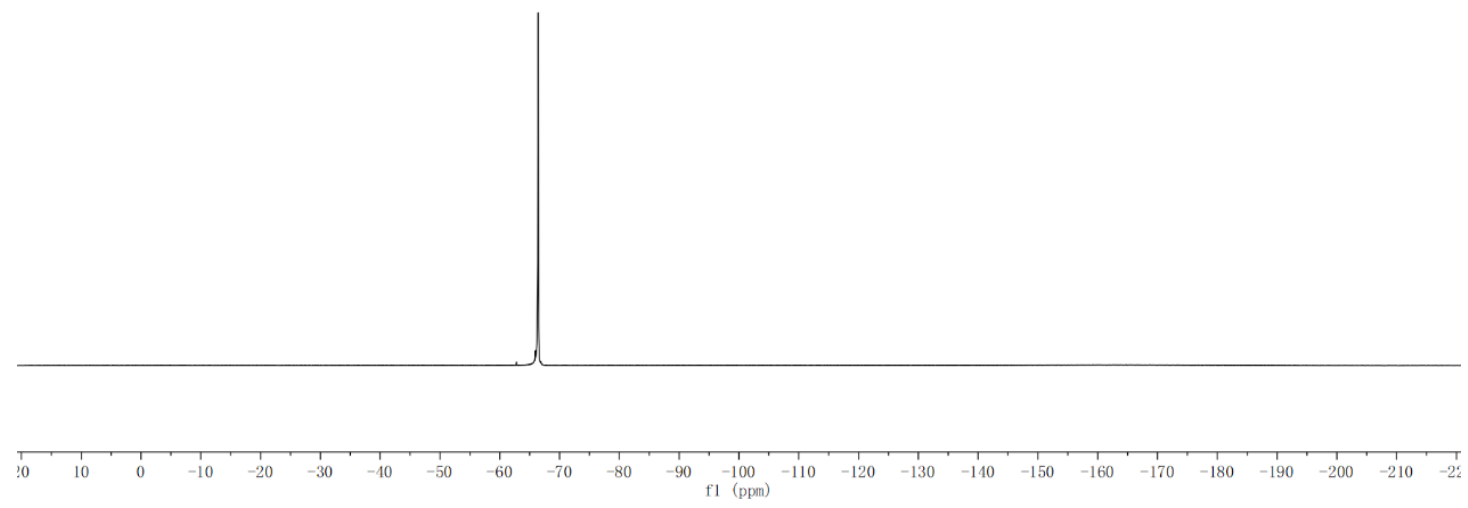

ethyl

(2R,3S,4S)-4-(2-((tert-butoxycarbonyl)amino)phenyl)-2-ethyl-5-oxo-3-(trifluoromethyl)pyrrolidi ne-3-carboxylate (3u-minor)<smiles>CCOC(=O)[O-]</smiles>

3u-minor was obtained as a yellow oil in 9\% yield $(6 \mathrm{~h}$ for Michael addition and $30 \mathrm{~h}$ for catalytic hydrogenation), $90 \%$ ee. The enantiomeric excess was determined by HPLC (Daicel Chiralpak AD-H, hexane/i-PrOH $=80: 20(\mathrm{v} / \mathrm{v}), \lambda=254 \mathrm{~nm}$, flow rate $\left.=1.0 \mathrm{~mL} / \mathrm{min}, 25^{\circ} \mathrm{C}\right)$ : $t_{\text {minor }}=5.03 \mathrm{~min}, t_{\text {major }}=3.53 \mathrm{~min} ;[\alpha]_{\mathrm{D}}{ }^{25} 21.71(c 0.13, \mathrm{MeOH}) ;{ }^{1} \mathrm{H} \mathrm{NMR}$ (500 MHz, Chloroform- $d$ ) $\delta 7.87(\mathrm{~d}, J=8.0 \mathrm{~Hz}, 1 \mathrm{H}), 7.71(\mathrm{~s}, 1 \mathrm{H}), 7.61$ (s, 1H), $7.28(\mathrm{t}, J=7.8 \mathrm{~Hz}, 1 \mathrm{H}), 7.21(\mathrm{~d}, J=8.0 \mathrm{~Hz}, 1 \mathrm{H}), 7.07(\mathrm{t}, J=7.6$ $\mathrm{Hz}, 1 \mathrm{H}), 4.71(\mathrm{~s}, 1 \mathrm{H}), 4.51-4.19(\mathrm{~m}, 2 \mathrm{H}), 4.05(\mathrm{dd}, J=10.6,3.4 \mathrm{~Hz}, 1 \mathrm{H}), 1.99-1.91(\mathrm{~m}, 1 \mathrm{H})$, $1.66(\mathrm{td}, J=18.4,17.7,9.6 \mathrm{~Hz}, 1 \mathrm{H}), 1.49(\mathrm{~s}, 9 \mathrm{H}), 1.33(\mathrm{t}, J=7.1 \mathrm{~Hz}, 3 \mathrm{H}), 1.02(\mathrm{t}, J=7.4 \mathrm{~Hz}, 3 \mathrm{H})$ ppm. ${ }^{13} \mathrm{C}$ NMR (125 MHz, Chloroform- $d$ ) $\delta 173.58,168.87,153.53,138.04,131.92,131.90$, $128.69,124.04$ (q, $J=286.3 \mathrm{~Hz}), 123.92,123.74,80.16,63.84,63.39$ (q, $J=23.8 \mathrm{~Hz}), 59.88$, 49.63, 28.33, 24.13, 13.84, $11.84 \mathrm{ppm} ;{ }^{19} \mathrm{~F}$ NMR (471 MHz, Chloroform- $d$ ) $\delta-59.99$ ppm. ESI-HRMS m/z: $445.1947[\mathrm{M}+\mathrm{H}]{ }^{+}, \mathrm{C}_{21} \mathrm{H}_{27} \mathrm{~F}_{3} \mathrm{~N}_{2} \mathrm{O}_{5}+\mathrm{H}^{+}$requires 445.1945. 

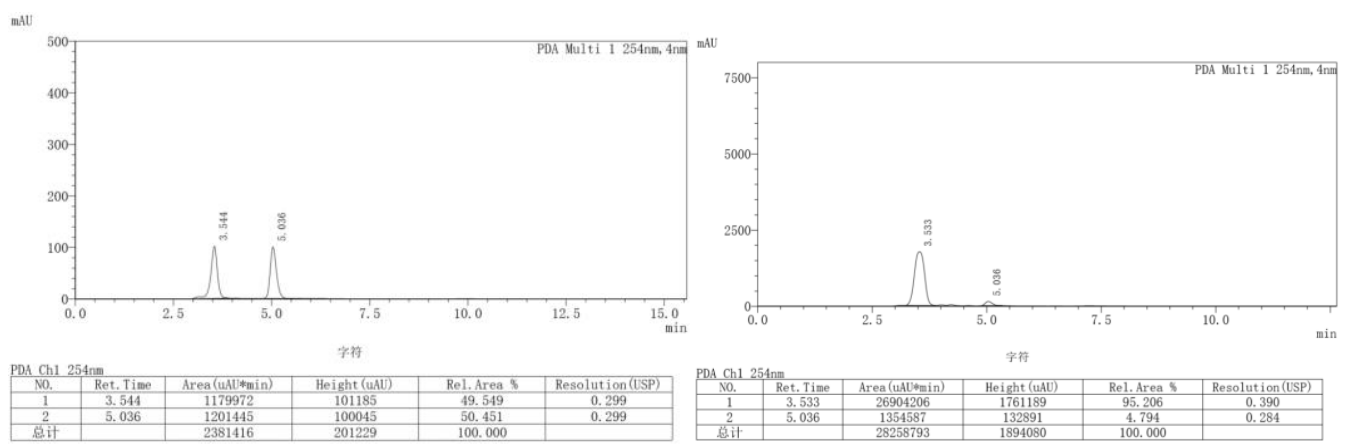

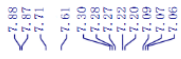

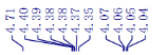

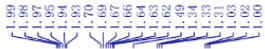

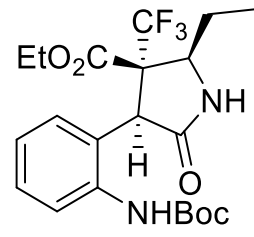

3u-minor

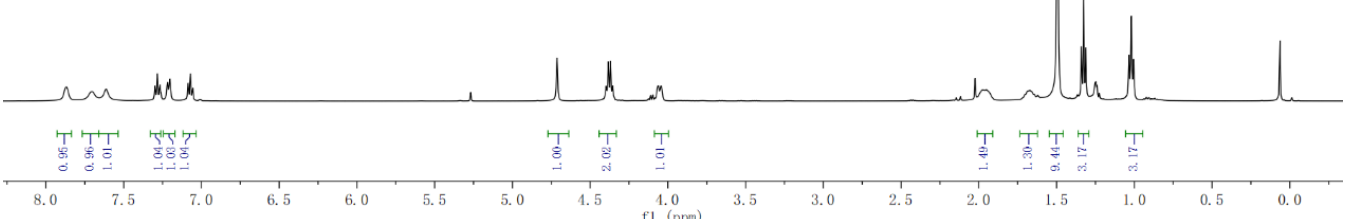



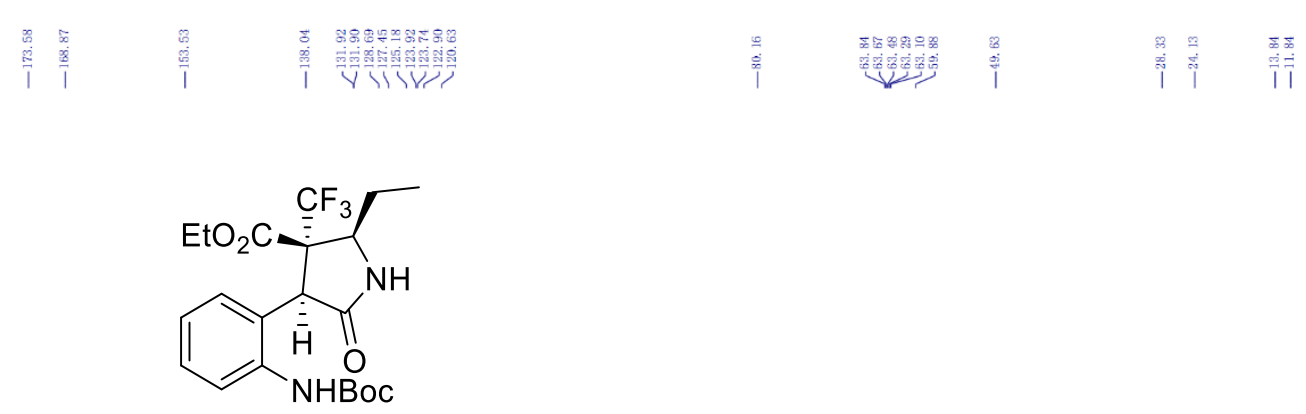

3u-minor
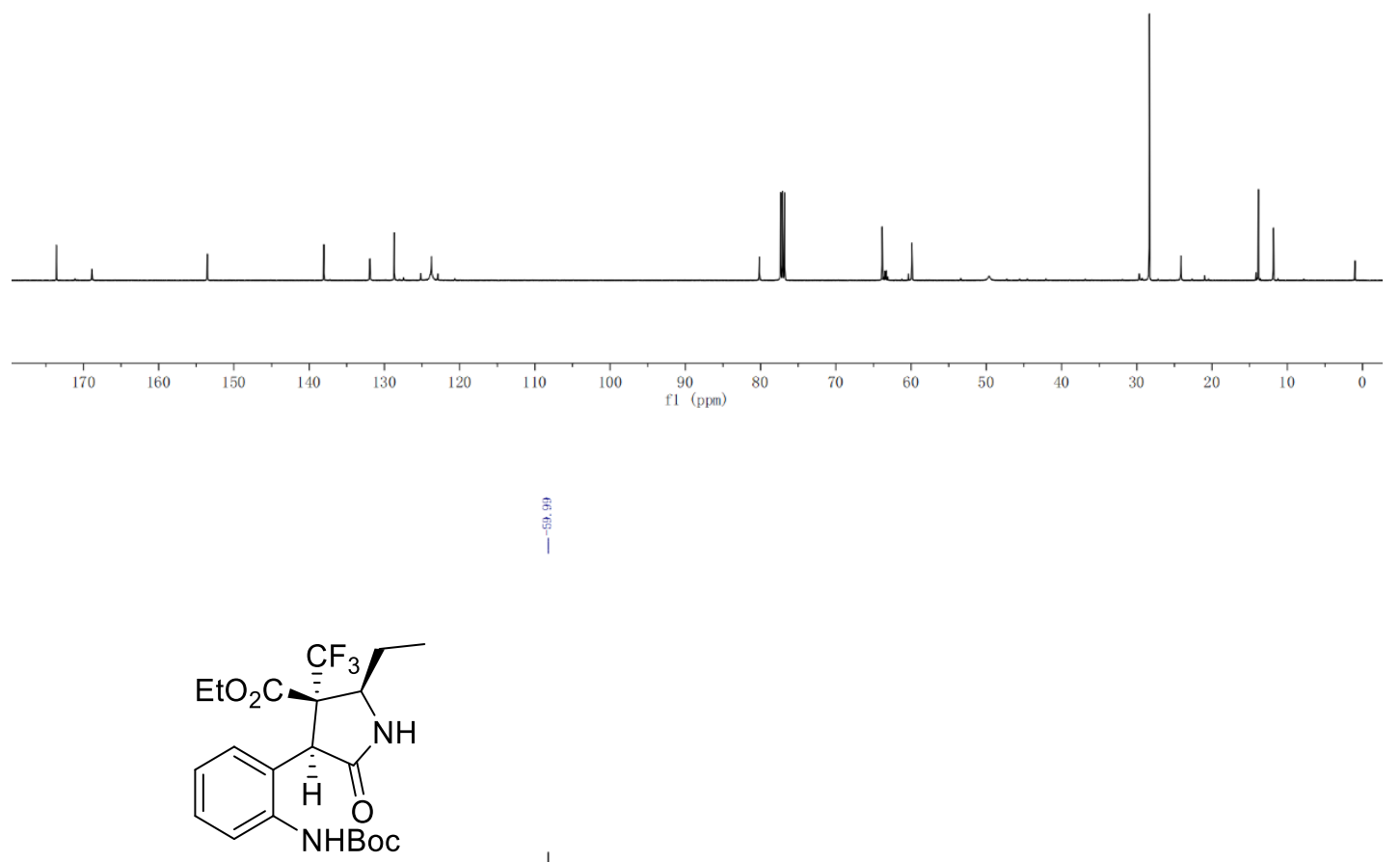

3u-minor

Note: The synthesis of $\mathbf{3 u}$ was also performed with $\mathbf{1 a}(0.1 \mathrm{mmol}, 1.0$ equiv.), $\mathbf{C 8}$ (10 mol\%), and 1-Nitropropane $(0.1 \mathrm{mmol}, 1.0$ equiv.) in DCM $(0.2 \mathrm{ml})$. Compared to the neat conditions, the time for Michael addition is extend to $36 \mathrm{~h}$. After catalytic hydrogenation, 3u was obtained with $43 \%$ yield, $88 \%$ ee and 3.5:1 dr. 


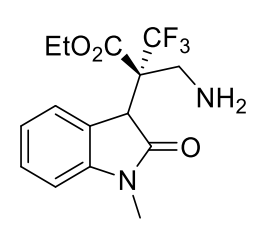

$3 \mathbf{v}$

3v was obtained as a yellow oil in $90 \%$ yield ( $5 \mathrm{~h}$ for Michael addition and $10 \mathrm{~h}$ for catalytic hydrogenation), 4.5:1 dr. We have tried our best to get the ee value of $3 \mathrm{v}$ with HPLC analysis, unfortunately, these stereoisomers could not be separated under present conditions. $[\alpha]_{\mathrm{D}}{ }^{25}-12.7($ c $0.23, \mathrm{MeOH}) ;{ }^{1} \mathrm{H}$ NMR (500 MHz, Chloroform- $d$ ) $\delta 7.40-7.26(\mathrm{~m}, 2 \mathrm{H}), 7.05(\mathrm{t}, J=7.6 \mathrm{~Hz}, 1 \mathrm{H})$, $6.84(\mathrm{~d}, J=7.9 \mathrm{~Hz}, 1 \mathrm{H}), 4.43(\mathrm{q}, J=7.2 \mathrm{~Hz}, 2 \mathrm{H}), 4.36(\mathrm{~s}, 1 \mathrm{H}), 3.31$ (d, $J=13.5$ $\mathrm{Hz}, 1 \mathrm{H}), 3.18$ (s, 3H), 3.12 (d, $J=13.6 \mathrm{~Hz}, 1 \mathrm{H}), 1.39$ (t, $J=7.2 \mathrm{~Hz}, 3 \mathrm{H}) \mathrm{ppm} .{ }^{13} \mathrm{C}$ NMR $(125 \mathrm{MHz}$, Chloroform- $d$ ) $\delta 173.77,167.92,144.40,128.81,125.75,124.01$ (q, $J=287.2 \mathrm{~Hz}), 123.72,122.48$, $108.24,62.50,60.36$ (q, $J=25.3 \mathrm{~Hz}), 45.31,29.67,26.30,13.93 \mathrm{ppm} ;{ }^{19} \mathrm{~F}$ NMR $(471 \mathrm{MHz}$, Chloroform- $d$ ) $\delta$-64.22 ppm. ESI-HRMS m/z: $331.1265[\mathrm{M}+\mathrm{H}]{ }^{+}, \mathrm{C}_{15} \mathrm{H}_{17} \mathrm{~F}_{3} \mathrm{~N}_{2} \mathrm{O}_{3}+\mathrm{H}^{+}$requires 331.1264 .
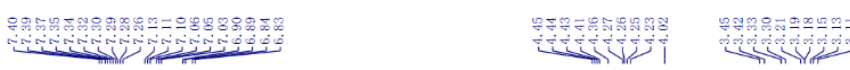<smiles>CCOC(=O)C(c1ccccc1NC)C(CN)C(=O)OC</smiles>

3v

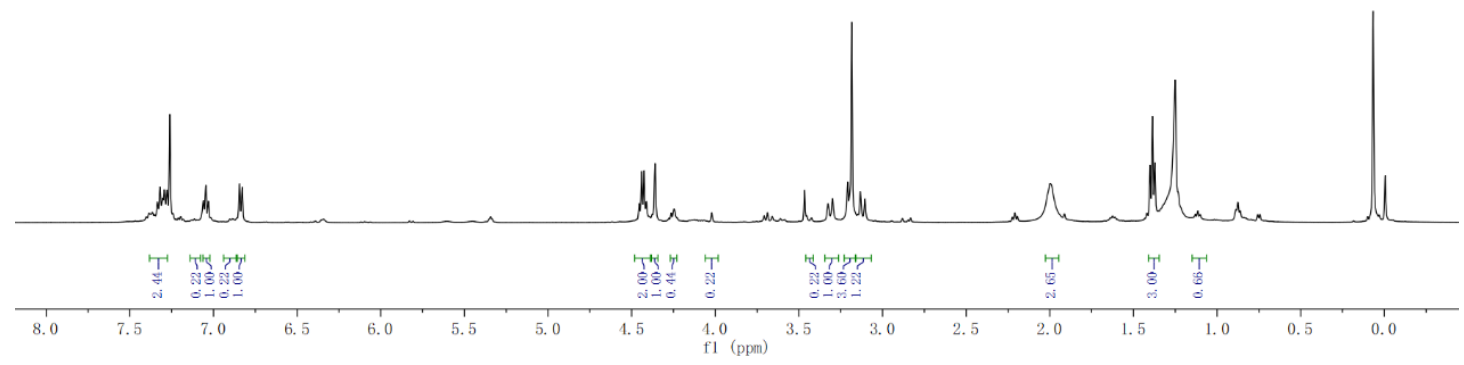



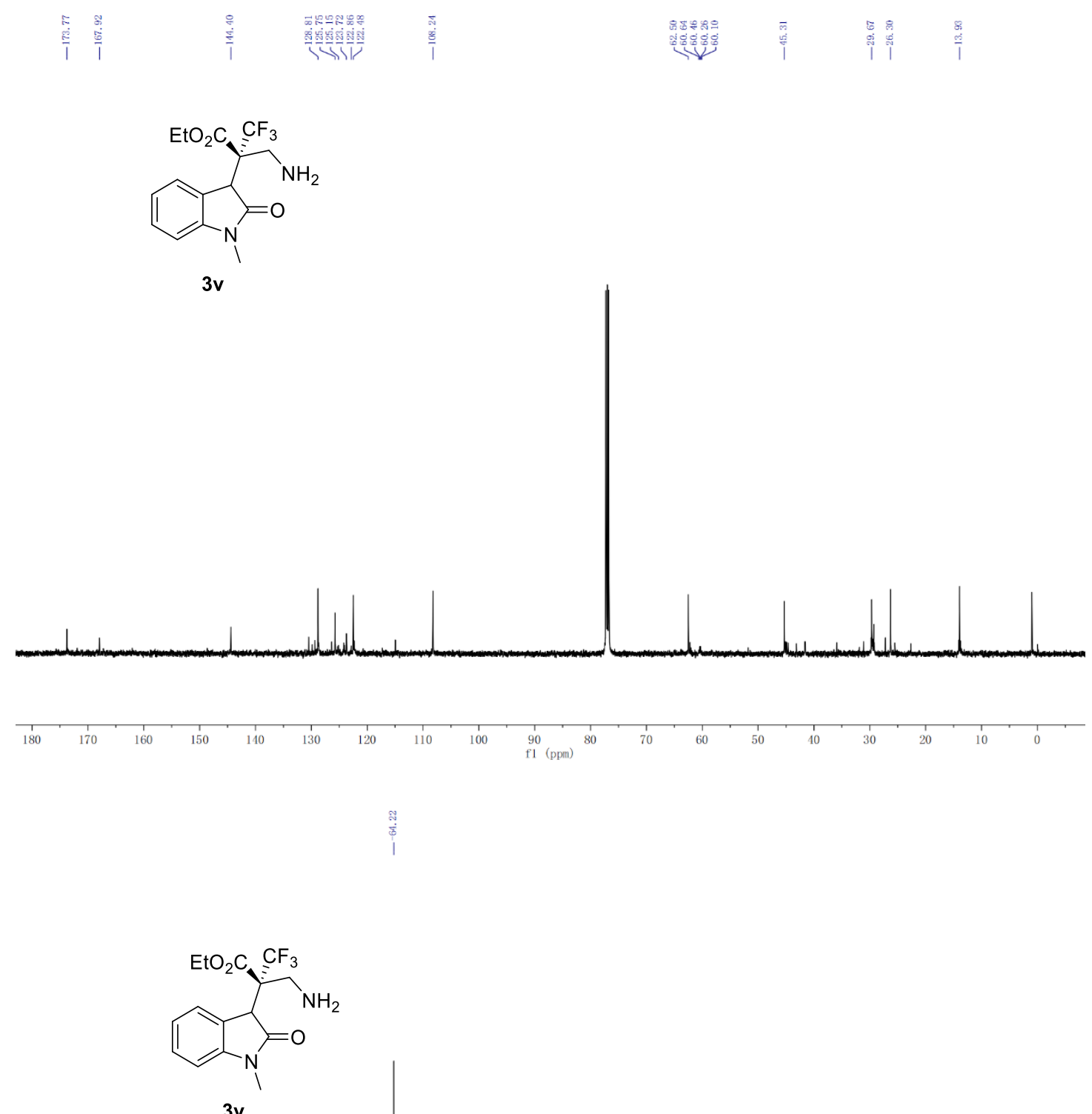

3v

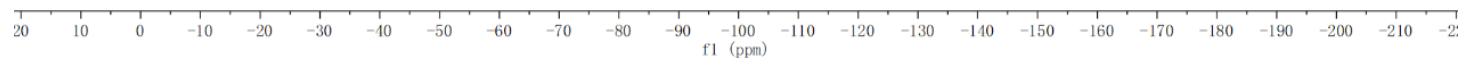




\section{Typical procedures and characterization data for compounds 4,5 and 6}

\section{Procedure for the synthesis of product 4}
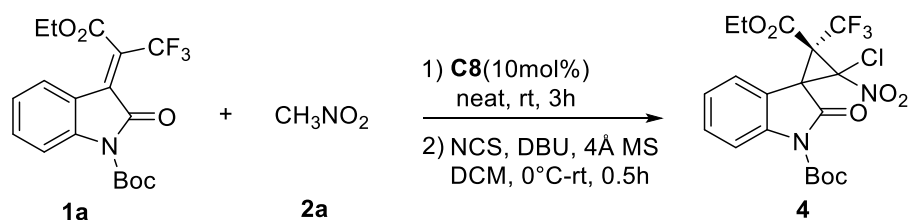

To a sample bottle equipped with a magnetic stirring bar, C8 (10 mol\%) and 2a (2 mmol, 20.0 equiv.) were stirred at room temperature under $\mathrm{N}_{2}$. Subsequently, 1a (0.1 mmol, 1.0 equiv.) was added in one portion. After completion (about 3 hours, monitored by TLC), the reaction mixture was directly concentrated under reduced pressure. The residue was dissolved in DCM (2.0 mL) at $0^{\circ} \mathrm{C}$. And $4 \AA$ molecular sieves $(20 \mathrm{mg})$, DBU (0.20 mmol, 2.0 equiv.) were added followed by NCS $\left(0.25 \mathrm{mmol}, 2.5\right.$ equiv.). Then the mixture was stirred from $0^{\circ} \mathrm{C}$ to room temperature until completion (about $0.5 \mathrm{~h}$, monitored by TLC). The reaction mixture was filtered through Celite. The filtrate was concentrated and the residue was purified by flash chromatography on silica gel (petroleum ether/ethyl acetate) to afford the product 4.

\section{Procedure for the synthesis of the racemic products 4}<smiles>CCOC(=O)C(=C1C(=O)N(C(C)=O)c2ccccc21)C(F)(F)C(F)(F)F</smiles>

1a<smiles>CNC(=O)O</smiles>

2a

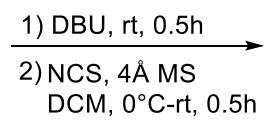
$\mathrm{DCM}, 0^{\circ} \mathrm{C}$-rt, $0.5 \mathrm{~h}$<smiles>CCOC(=O)c1ccccc1C1(C(F)(F)F)C(=O)N(c2ccccc2)C1(Cl)C(F)(F)F</smiles>

4

To a sample bottle equipped with a magnetic stirring bar, $1 \mathbf{a}(0.1 \mathrm{mmol}, 1.0$ equiv. $)$ was added to a solution of DBU ( $0.01 \mathrm{mmol}, 0.1$ equiv.) and $\mathbf{2 a}(1 \mathrm{mmol}, 10.0$ equiv.) in DCM (2.0 mL) at room temperature under $\mathrm{N}_{2}$. After $0.5 \mathrm{~h}$, the reaction mixture was concentrated under reduced pressure. The residue was dissolved in DCM $(2.0 \mathrm{~mL})$ at $0^{\circ} \mathrm{C}$. And $4 \AA$ molecular sieves $(20 \mathrm{mg})$, DBU (0.20 mmol, 2.0 equiv.) were added followed by NCS (0.25 mmol, 2.5 equiv.). Then the mixture was stirred from $0^{\circ} \mathrm{C}$ to room temperature until completion (about $0.5 \mathrm{~h}$, monitored by TLC). The reaction mixture was filtered through Celite. The filtrate was concentrated and the residue was purified by flash chromatography on silica gel (petroleum ether/ethyl acetate) to afford the racemic compounds 4 .

\section{1'-(tert-butyl) 2-ethyl}

(2S)-3-chloro-3-nitro-2'-oxo-2-(trifluoromethyl)spiro[cyclopropane-1,3'-indoline]-1',2-dicarbox ylate (4)

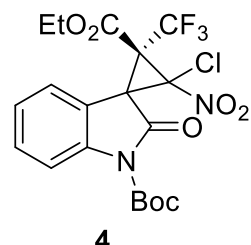

4 was obtained as a yellow oil in $60 \%$ yield $(3.5 \mathrm{~h}), 95 \%$ ee and $>20: 1 \mathrm{dr}$. The enantiomeric excess was determined by HPLC (Daicel Chiralpak OD-H, hexane $/ \mathrm{i}-\mathrm{PrOH}=90: 10(\mathrm{v} / \mathrm{v}), \lambda=254 \mathrm{~nm}$, flow rate $\left.=0.5 \mathrm{~mL} / \mathrm{min}, 25^{\circ} \mathrm{C}\right)$ : $t_{\text {minor }}=12.02 \mathrm{~min}, t_{\text {major }}=10.37 \mathrm{~min} ;[\alpha]_{\mathrm{D}}{ }^{25}-65.1(c 0.27, \mathrm{MeOH}) ;{ }^{1} \mathrm{H} \mathrm{NMR}$ (400 MHz, Chloroform- $d$ ) $\delta 7.98(\mathrm{~d}, J=8.3 \mathrm{~Hz}, 1 \mathrm{H}), 7.62(\mathrm{~d}, J=7.9 \mathrm{~Hz}, 1 \mathrm{H})$, $7.54(\mathrm{t}, J=8.0 \mathrm{~Hz}, 1 \mathrm{H}), 7.27(\mathrm{t}, J=7.8 \mathrm{~Hz}, 1 \mathrm{H}), 4.30(\mathrm{q}, J=7.1 \mathrm{~Hz}, 2 \mathrm{H})$, 
$1.61(\mathrm{~s}, 9 \mathrm{H}), 1.27(\mathrm{t}, J=7.1 \mathrm{~Hz}, 3 \mathrm{H}) \mathrm{ppm} .{ }^{13} \mathrm{C}$ NMR $(125 \mathrm{MHz}$, Chloroform- $d$ ) $\delta$ 169.09, 161.32, 148.01, 141.76, 133.24, 127.58, 125.14, 122.23 (q, $J=288.8 \mathrm{~Hz}$ ), 117.04, 115.77, 85.75, 81.34, 67.15 (q, $J=28.4 \mathrm{~Hz}), 64.60,27.96,18.41,13.36 \mathrm{ppm} .{ }^{19} \mathrm{~F}$ NMR $(471 \mathrm{MHz}$, Chloroform- $d$ ) $\delta$ -60.32 ppm. ESI-HRMS m/z: $479.0829[\mathrm{M}+\mathrm{H}]{ }^{+}, \mathrm{C}_{19} \mathrm{H}_{18} \mathrm{ClF}_{3} \mathrm{~N}_{2} \mathrm{O}_{7}+\mathrm{H}^{+}$requires 479.0827.
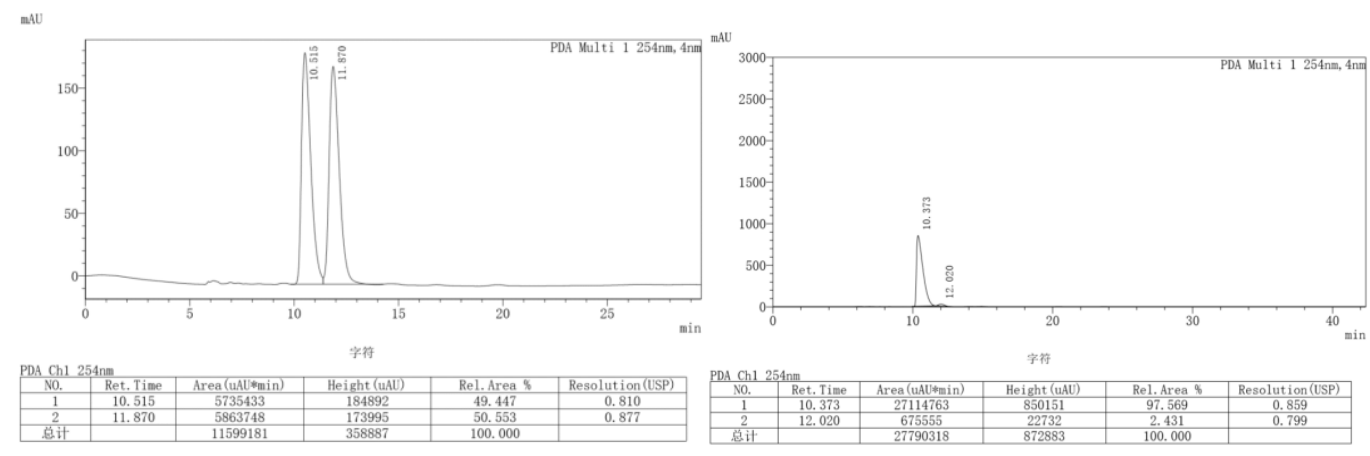

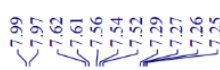

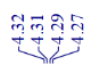

本<smiles>CCOC(=O)c1ccccc1N1C(=O)C2(C(F)(F)F)c3ccccc3C1(C(F)(F)F)C2(Cl)Cl</smiles>

4

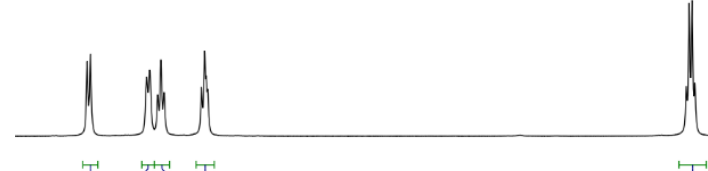

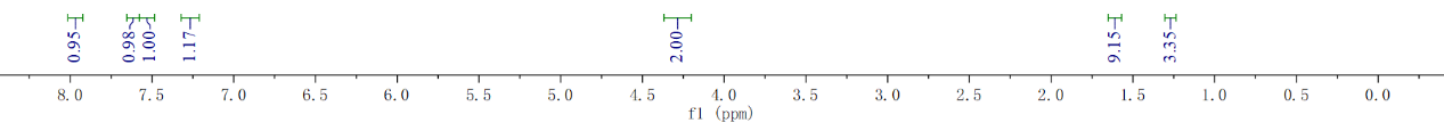




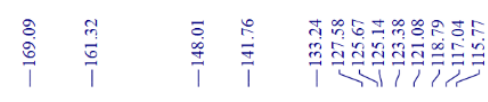

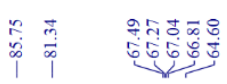

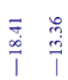

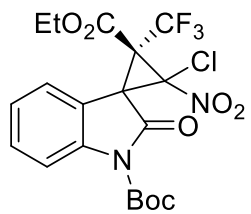

4

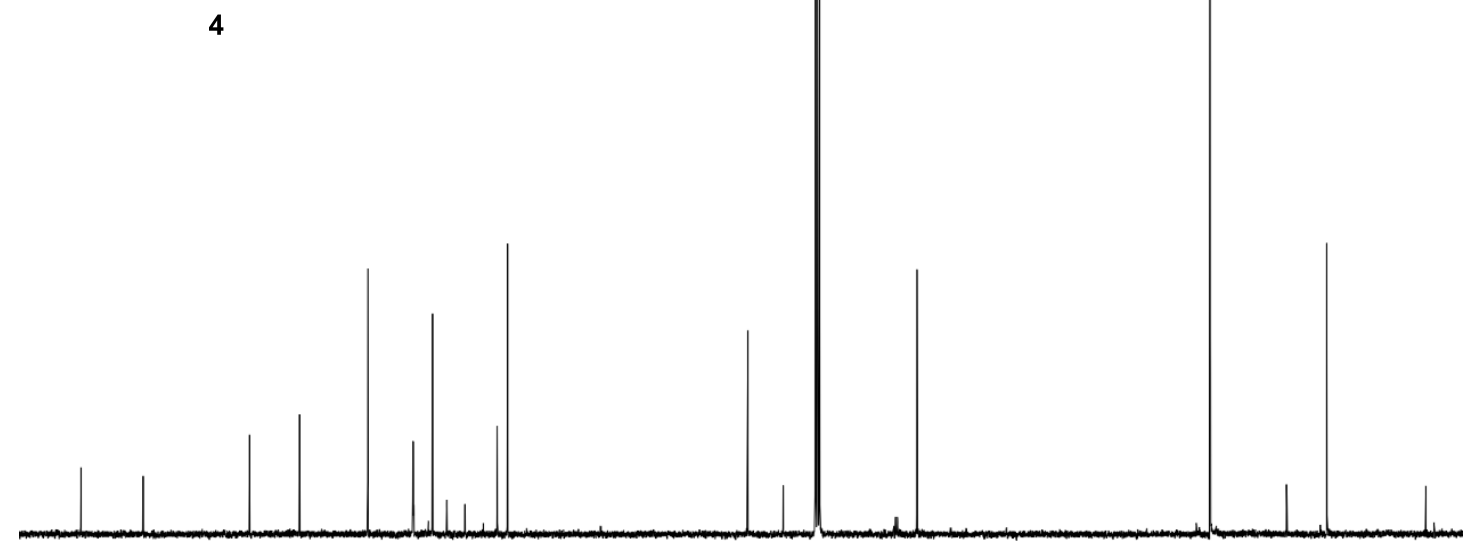<smiles>CCOC(=O)N1C(=O)C2(c3ccccc31)C(O)([N+](=O)[O-])[C@@]2(C(=O)OCC)C(F)(F)Cl</smiles>

4

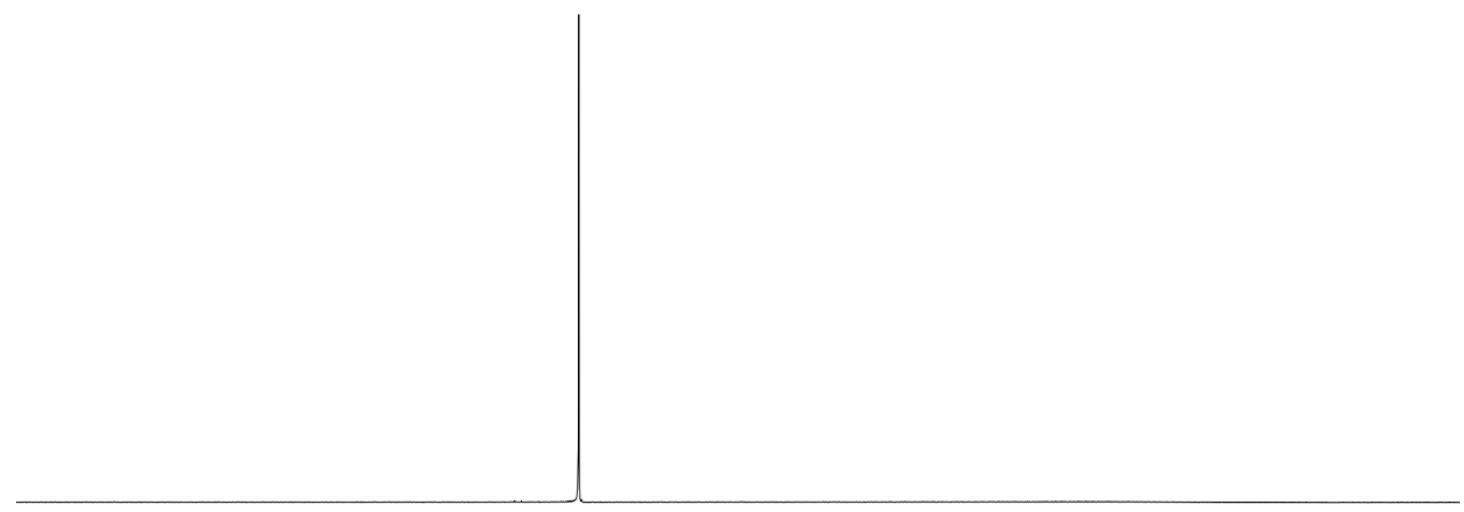

30
30 
Procedure for the synthesis of product 5

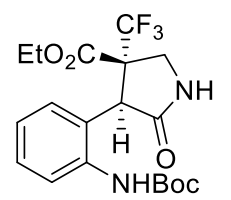

$3 a$<smiles>O=C1CC2(C(=O)N1)C(=O)Nc1ccccc12</smiles>

To a sample bottle equipped with a magnetic stirring bar, 3a $(0.1 \mathrm{mmol}, 1.0$ equiv.) was dissolved in dichloromethane $(0.5 \mathrm{~mL})$, and $\mathrm{CF}_{3} \mathrm{COOH}(0.5 \mathrm{mmol}, 5.0$ equiv. $)$ was added. The mixture was stirred at room temperature under $\mathrm{N}_{2}$ for $1.0 \mathrm{~h}$, then the solution was removed under reduced pressure and the residue was purified by flash chromatography on silica gel (petroleum ether/ethyl acetate) to afford the product 5 .

(3aS,9bS)-3a-(trifluoromethyl)-3,3a,5,9b-tetrahydro-1H-pyrrolo[3,4-c]

dione (5)<smiles>O=C1NC[C@]2(C(F)(F)F)C(=O)Nc3ccccc3[C@H]12</smiles>

5

5 was obtained as a yellow foam in $88 \%$ yield $(1.0 \mathrm{~h}), 97 \%$ ee and $>20: 1 \mathrm{dr}$. The enantiomeric excess was determined by HPLC (Daicel Chiralpak AD-H, hexane $/ \mathrm{i}-\mathrm{PrOH}=80: 20(\mathrm{v} / \mathrm{v}), \lambda=254 \mathrm{~nm}$, flow rate $\left.=1.0 \mathrm{~mL} / \mathrm{min}, 25^{\circ} \mathrm{C}\right): t_{\text {minor }}$ $=10.98 \mathrm{~min}, t_{\text {major }}=6.32 \mathrm{~min} ;[\alpha]_{\mathrm{D}}{ }^{25} 24.5(c 0.58, \mathrm{MeOH}) ;{ }^{1} \mathrm{H}$ NMR $(400 \mathrm{MHz}$, Chloroform- $d$ ) $\delta 10.89(\mathrm{~s}, 1 \mathrm{H}), 8.42(\mathrm{~s}, 1 \mathrm{H}), 7.33(\mathrm{~d}, \mathrm{~J}=7.3 \mathrm{~Hz}, 1 \mathrm{H}), 7.25(\mathrm{t}, \mathrm{J}$ $=7.3 \mathrm{~Hz}, 1 \mathrm{H}), 7.13(\mathrm{t}, \mathrm{J}=7.4 \mathrm{~Hz}, 1 \mathrm{H}), 6.88(\mathrm{~d}, \mathrm{~J}=7.3 \mathrm{~Hz}, 1 \mathrm{H}), 4.24(\mathrm{~d}, \mathrm{~J}=$ $10.2 \mathrm{~Hz}, 1 \mathrm{H}), 4.07(\mathrm{~s}, 1 \mathrm{H}), 3.65(\mathrm{~d}, \mathrm{~J}=10.4 \mathrm{~Hz}, 1 \mathrm{H}) \mathrm{ppm} .{ }^{13} \mathrm{C}$ NMR $(125$ MHz, Chloroform- $d$ ) $\delta$ 173.62, 164.28, 134.48, 129.94, 129.34, 124.42, 124.18 (q, $J=282.0 \mathrm{~Hz}$ ), 116.32, 115.68, 53.36 (q, $J=26.7 \mathrm{~Hz}), 45.53,44.43 \mathrm{ppm} .{ }^{19} \mathrm{~F}$ NMR (471 MHz, Chloroform- $d$ ) $\delta$ -71.29 ppm. ESI-HRMS m/z: $271.0692[\mathrm{M}+\mathrm{H}]^{+}, \mathrm{C}_{12} \mathrm{H}_{9} \mathrm{~F}_{3} \mathrm{~N}_{2} \mathrm{O}_{2}+\mathrm{H}^{+}$requires 271.0694.
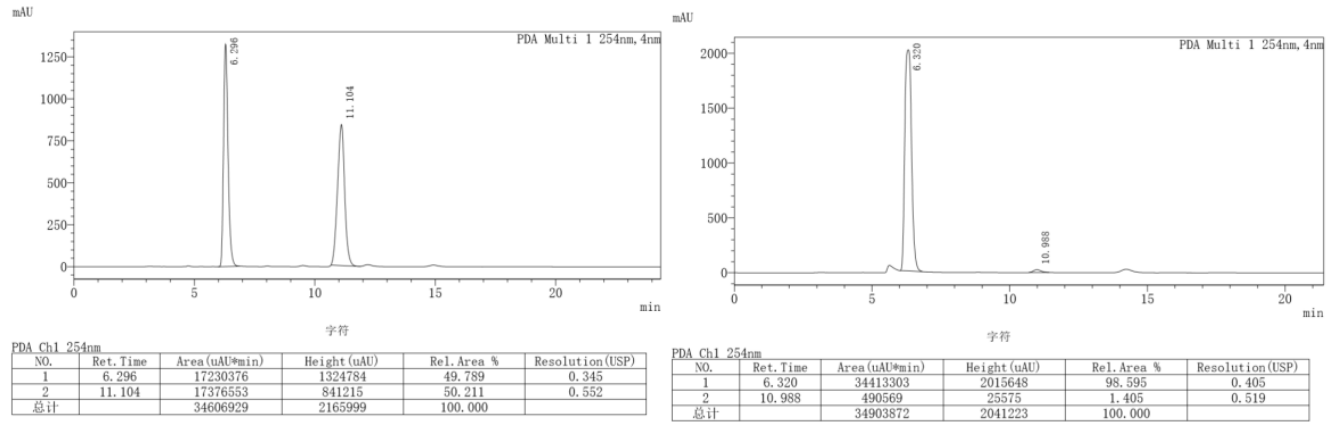


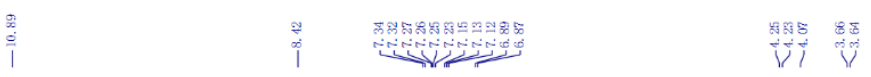<smiles>C[C@@]12C(=O)NCC1(C(F)(F)F)C(=O)Nc1ccccc12</smiles>

5

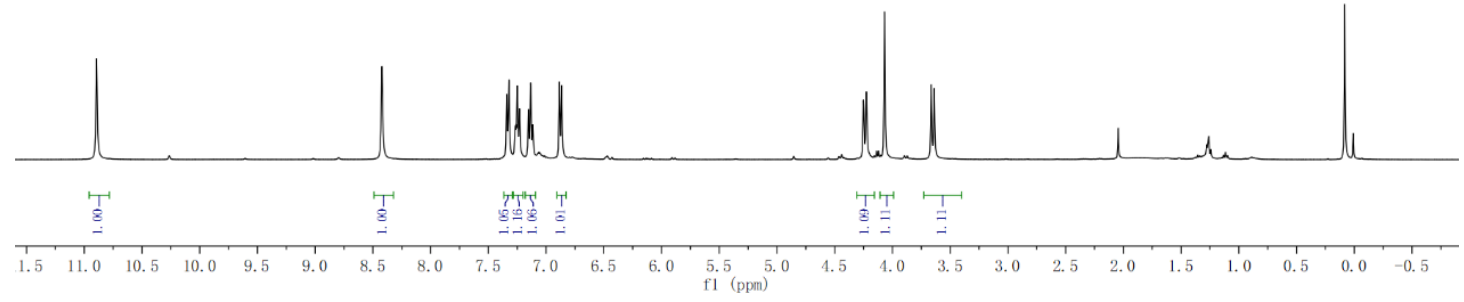

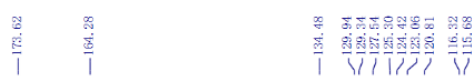

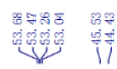<smiles>O=C1NCC2(C(F)(F)F)C(=O)Nc3ccccc3[C@H]12</smiles>

5
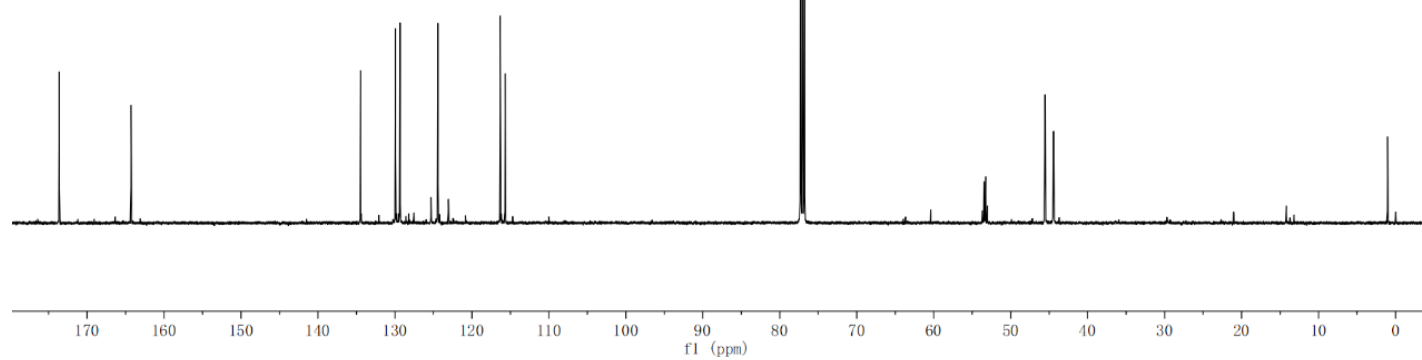
<smiles>O=C1NC(=O)C2(C(F)(F)F)C(=O)NCC12CF</smiles>

5

Procedure for the synthesis of products 6 and 6 '

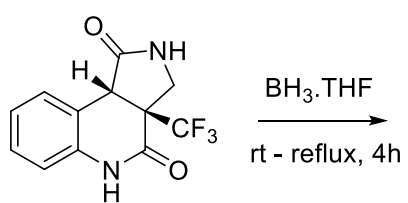

5<smiles>FC1(F)CNCC12CNCC21CCCC1</smiles>

6<smiles>FC(F)(F)C12CNCC1c1ccccc1NC2</smiles>

$6^{\prime}$

To a solution of 5 ( $0.1 \mathrm{mmol}, 1.0$ equiv. $)$ in anhydrous THF $(2 \mathrm{~mL})$ was slowly added $1.0 \mathrm{M}$ borane tetrahydrofuran complex $\left(0.5 \mathrm{mmol}, 5.0\right.$ equiv.) at room temperature under $\mathrm{N}_{2}$. Then the mixture was refluxed for 4 hours. The solvent was removed under reduced pressure and the residue was purified by silica gel column chromatography (petroleum ether/ethyl acetate) to afford the compounds $\mathbf{6}$ and $\mathbf{6}$ '.

(3aS,9bS)-3a-(trifluoromethyl)-2,3,3a,4,5,9b-hexahydro-1H-pyrrolo[3,4-c] quinoline (6)<smiles>FC(F)(F)C12CNCC1CNc1ccccc12</smiles>

6

6 was obtained as a yellow foam in 35\% yield $(4.0 \mathrm{~h}), 97 \%$ ee. The enantiomeric excess was determined by HPLC (Daicel Chiralpak AD-H, hexane/i-PrOH $=80: 20(\mathrm{v} / \mathrm{v}), \lambda=254 \mathrm{~nm}$, flow rate $\left.=1.0 \mathrm{~mL} / \mathrm{min}, 25{ }^{\circ} \mathrm{C}\right)$ : $t_{\text {minor }}=5.82 \mathrm{~min}, t_{\text {major }}=7.77 \mathrm{~min} ;[\alpha]_{\mathrm{D}}^{25} 38.6(c 0.41, \mathrm{MeOH})$;

${ }^{1} \mathrm{H}$ NMR (400 MHz, Chloroform-d) $\delta 7.12(\mathrm{td}, J=7.7,1.5 \mathrm{~Hz}, 1 \mathrm{H}), 7.03$ (dd, $J=7.7,1.5 \mathrm{~Hz}, 1 \mathrm{H}), 6.83(\mathrm{td}, J=7.5,1.2 \mathrm{~Hz}, 1 \mathrm{H}), 6.74(\mathrm{dd}, \mathrm{J}=7.5,1.2 \mathrm{~Hz}$, 1H), 4.32 (s, 1H), 3.80 (s, 1H), $3.73-3.60(\mathrm{~m}, 2 \mathrm{H}), 3.53$ (dd, $J=10.8,8.1 \mathrm{~Hz}, 1 \mathrm{H}), 3.31-3.16$ $(\mathrm{m}, 2 \mathrm{H}), 3.10(\mathrm{~d}, J=11.8 \mathrm{~Hz}, 1 \mathrm{H}), 2.61$ (q, $J=11.8 \mathrm{~Hz}, 1 \mathrm{H}) \mathrm{ppm} .{ }^{13} \mathrm{C}$ NMR $(125 \mathrm{MHz}$, Chloroform- $d$ ) $\delta 145.14,128.70,128.23,127.77$ (q, $J=281.4 \mathrm{~Hz}$ ), 122.39, 120.51, 116.10, 62.15, 
$58.71,54.43(\mathrm{q}, J=23.8 \mathrm{~Hz}), 45.14,41.05 \mathrm{ppm} .{ }^{19} \mathrm{~F}$ NMR $(471 \mathrm{MHz}$, Chloroform- $d$ ) $\delta-75.60 \mathrm{ppm}$. ESI-HRMS m/z: $243.1107[\mathrm{M}+\mathrm{H}]^{+}, \mathrm{C}_{12} \mathrm{H}_{13} \mathrm{~F}_{3} \mathrm{~N}_{2}+\mathrm{H}^{+}$requires 243.1103 .
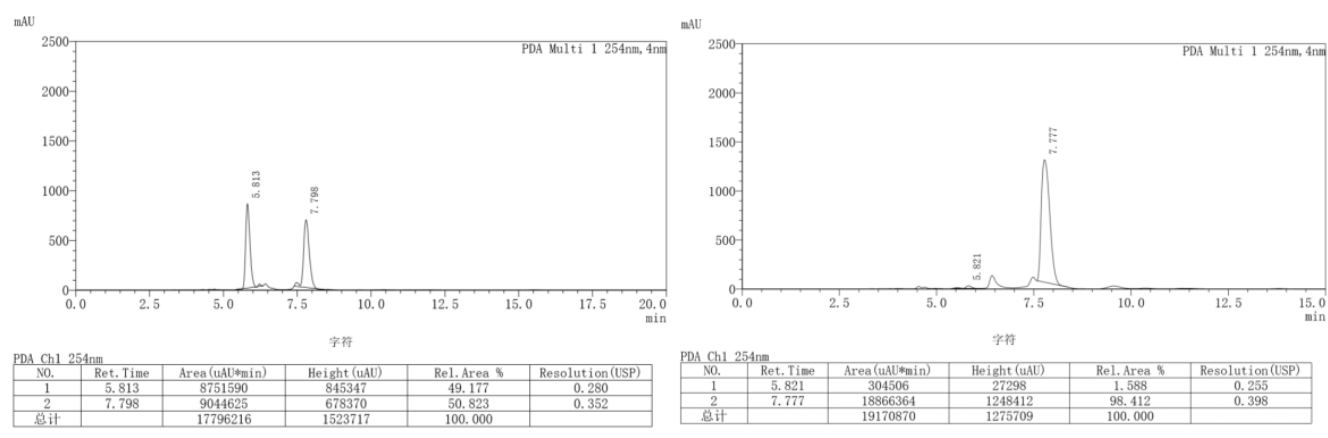

$\underbrace{209=0}$

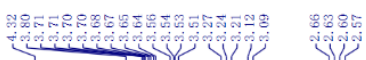<smiles>FC1(F)CNCC12CNCC21CNCc2ccccc21</smiles>
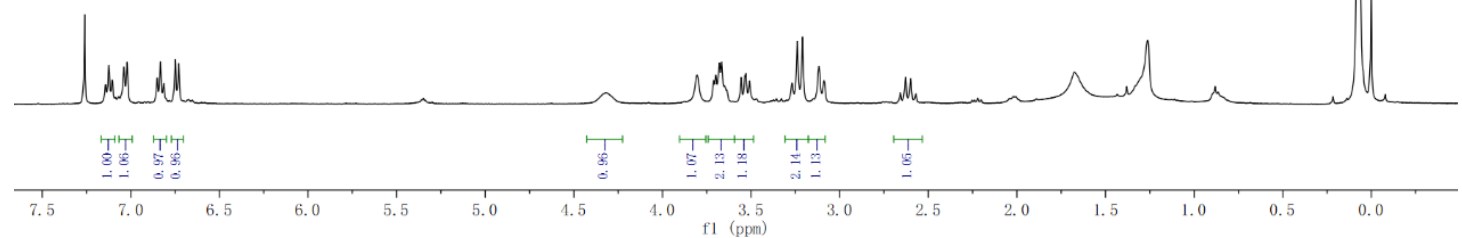

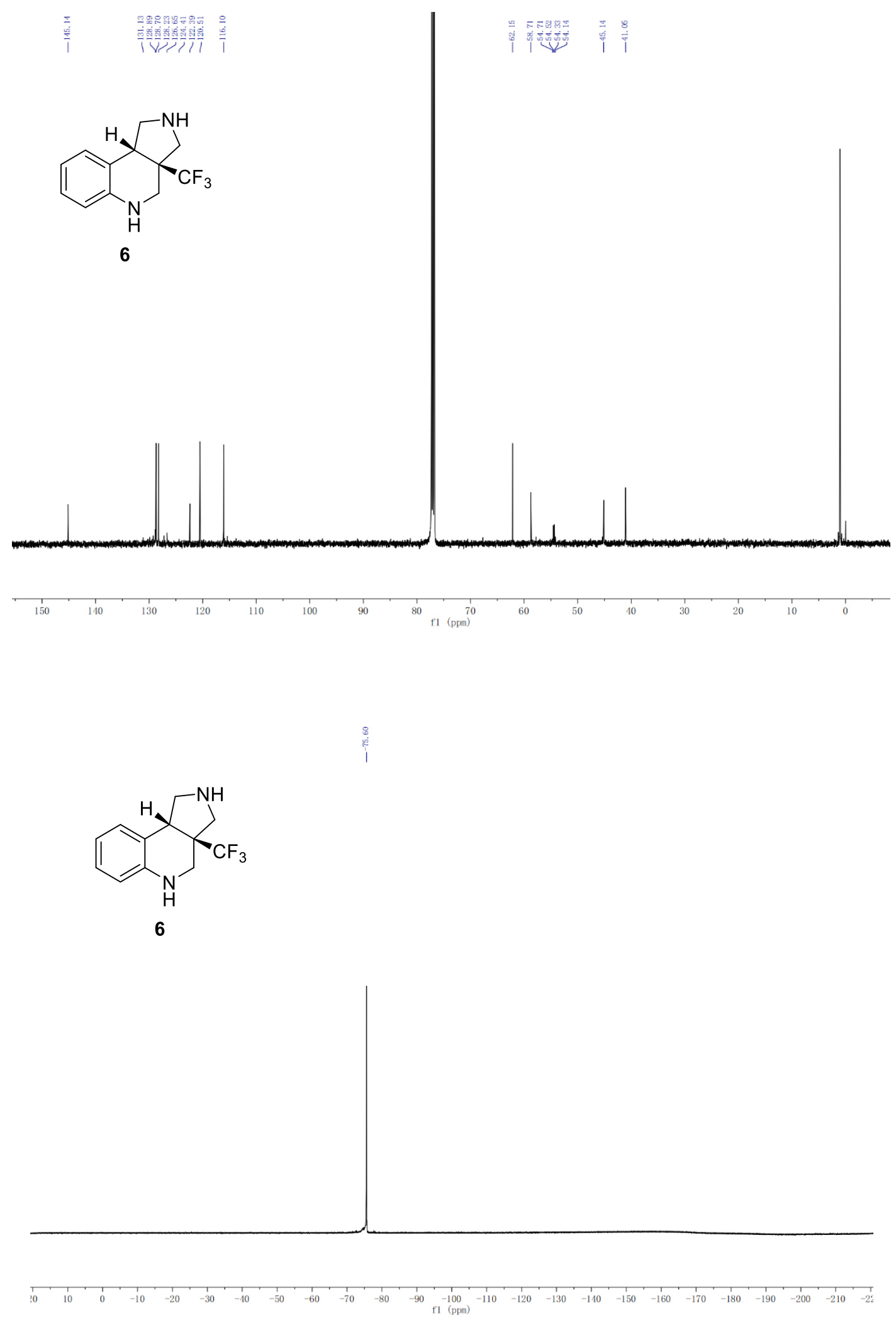
6' was obtained as a white foam in $33 \%$ yield $(4.0 \mathrm{~h}), 97 \%$ ee. The enantiomeric excess was determined by HPLC (Daicel Chiralpak OD-H,<smiles>FC(F)(F)C12CNCC1CNc1ccccc12</smiles>

6 hexane/i-PrOH $=80: 20(\mathrm{v} / \mathrm{v}), \lambda=254 \mathrm{~nm}$, flow rate $\left.=1.0 \mathrm{~mL} / \mathrm{min}, 25{ }^{\circ} \mathrm{C}\right)$ : $t_{\text {minor }}=12.49 \mathrm{~min}, t_{\text {major }}=9.28 \mathrm{~min} ;[\alpha]_{\mathrm{D}}{ }^{25}-17.2(c 0.38, \mathrm{MeOH}) ;{ }^{1} \mathrm{H} \mathrm{NMR}$ (400 MHz, Chloroform- $d$ ) $\delta 7.20-7.00(\mathrm{~m}, 2 \mathrm{H}), 6.91(\mathrm{~d}, J=7.5 \mathrm{~Hz}, 1 \mathrm{H})$, $6.69(\mathrm{~d}, J=8.0 \mathrm{~Hz}, 1 \mathrm{H}), 4.28(\mathrm{~s}, 1 \mathrm{H}), 3.81(\mathrm{~s}, 1 \mathrm{H}), 3.69(\mathrm{~d}, J=6.2 \mathrm{~Hz}, 1 \mathrm{H})$, $3.51(\mathrm{dd}, J=13.3,5.9 \mathrm{~Hz}, 1 \mathrm{H}), 3.35-3.19(\mathrm{~m}, 4 \mathrm{H}), 3.14(\mathrm{dd}, J=12.2,4.2$ $\mathrm{Hz}, 1 \mathrm{H}) \mathrm{ppm} .{ }^{13} \mathrm{C}$ NMR $(125 \mathrm{MHz}$, Chloroform- $d$ ) $\delta 146.08,129.44,128.33,127.05$ (q, $J=$ $280.8 \mathrm{~Hz}$ ), 123.92, 121.55, 115.92, 65.09, 60.00, 54.38 (q, $J=25.0 \mathrm{~Hz}), 46.03,41.70 \mathrm{ppm} .{ }^{19} \mathrm{~F}$ NMR (471 MHz, Chloroform- $d$ ) $\delta-75.67$ ppm. ESI-HRMS m/z: $243.1105[\mathrm{M}+\mathrm{H}]{ }^{+}$, $\mathrm{C}_{12} \mathrm{H}_{13} \mathrm{~F}_{3} \mathrm{~N}_{2}+\mathrm{H}^{+}$requires 243.1103 .
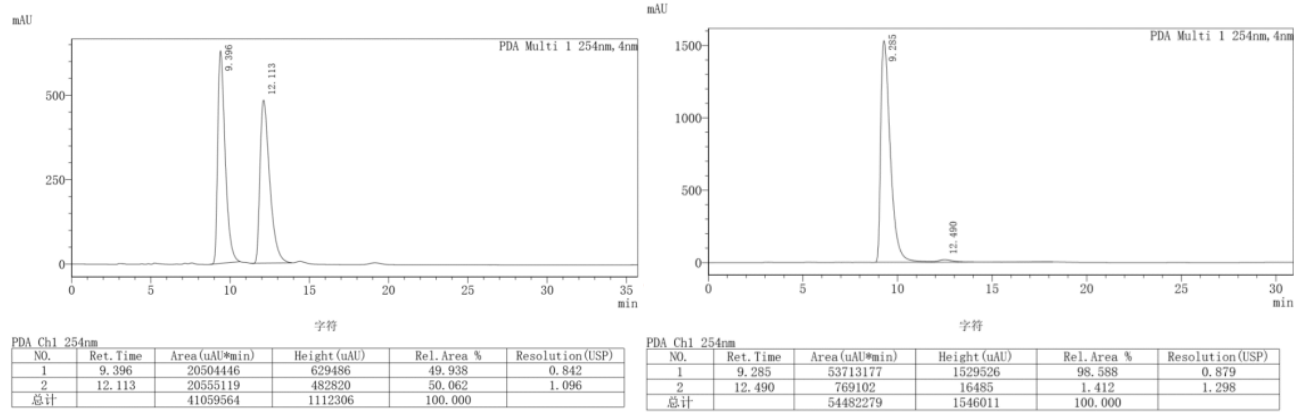<smiles>FC(F)(F)C12CNCC1c1ccccc1NC2</smiles>

$6^{\prime}$

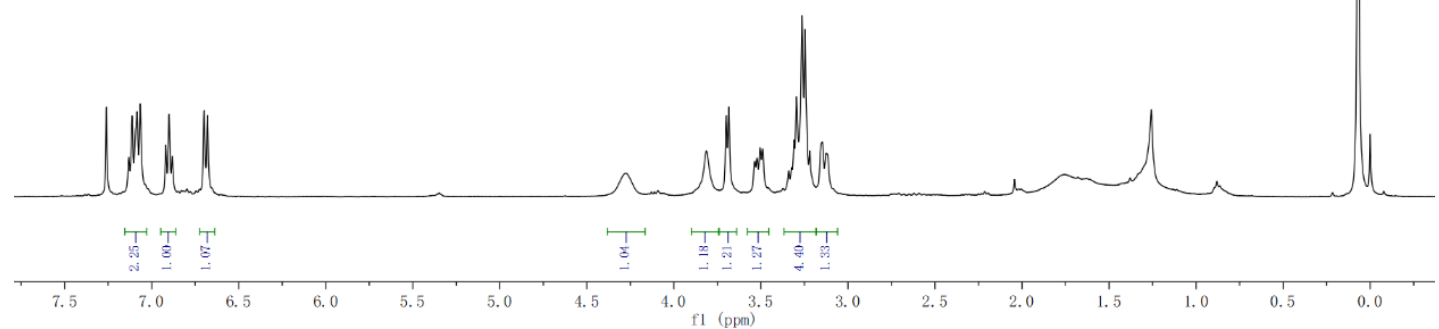




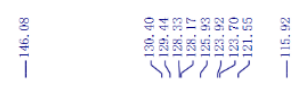
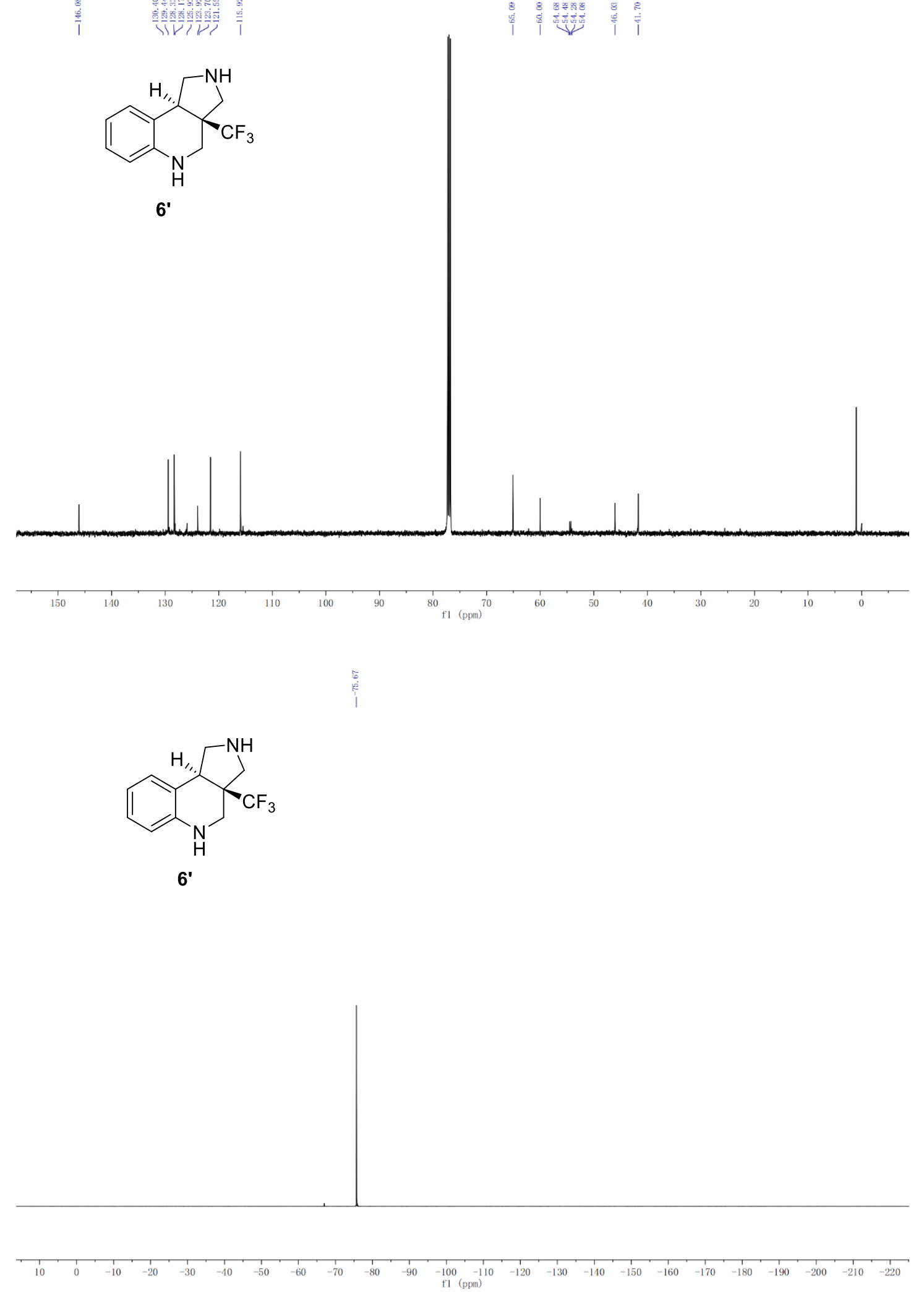
4. X-ray data of 3a
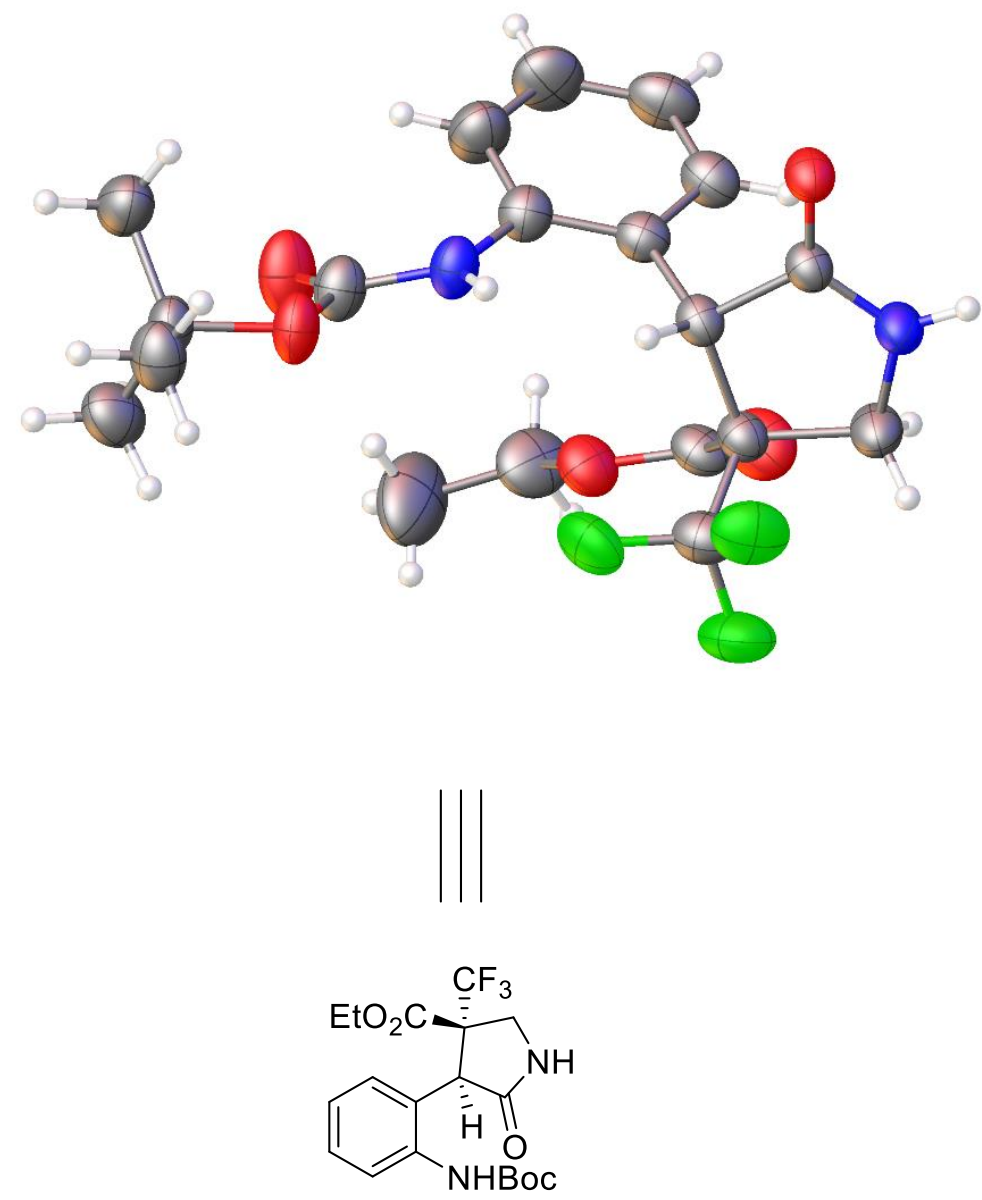

$3 a$ 
Table 1. Crystal data and structure refinement for 3a.

Identification code

Empirical formula

Formula weight

Temperature

Wavelength

Crystal system, space group

Unit cell dimensions

Volume

Z, Calculated density

Absorption coefficient

F (000)

Crystal size

Theta range for data collection

Limiting indices

Reflections collected / unique

Completeness to theta $=66.849$

Absorption correction

Max. and min. transmission

Refinement method

Data / restraints / parameters

Goodness-of-fit on $\mathrm{F}^{\wedge} 2$

Final R indices [I $>2 \operatorname{sigma}(\mathrm{I})]$

$\mathrm{R}$ indices (all data)

Absolute structure parameter

Extinction coefficient

Largest diff. peak and hole 3a

C19 H23 F3 N2 O5

416.39

166(4) K

$1.54184 \mathrm{~A}$

Monoclinic, P 1211

$\mathrm{a}=17.34100(10)$ A $\quad$ alpha $=90 \mathrm{deg}$.

$\mathrm{b}=14.45120(10) \mathrm{A} \quad$ beta $=92.8490(10) \mathrm{deg}$.

$\mathrm{c}=18.20780(10) \mathrm{A} \quad$ gamma $=90 \mathrm{deg}$.

4557.20(5) $\mathrm{A}^{\wedge} 3$

$8,1.214 \mathrm{Mg} / \mathrm{m}^{\wedge} 3$

$0.892 \mathrm{~mm}^{\wedge}-1$

1744

$0.25 \times 0.21 \times 0.14 \mathrm{~mm}$

3.611 to $66.849 \mathrm{deg}$.

$-20<=\mathrm{h}<=20,-16<=\mathrm{k}<=17,-21<=1<=20$

$75547 / 15847[\mathrm{R}(\mathrm{int})=0.0304]$

$99.3 \%$

Semi-empirical from equivalents

1.00000 and 0.89580

Full-matrix least-squares on $\mathrm{F}^{\wedge} 2$

15847 / 17 / 1061

1.055

$\mathrm{R} 1=0.0368, \mathrm{wR} 2=0.1144$

$\mathrm{R} 1=0.0385, \mathrm{wR} 2=0.1165$

$-0.03(3)$

$\mathrm{n} / \mathrm{a}$

0.447 and -0.222 e. $\mathrm{A}^{\wedge}-3$

Table 2. Atomic coordinates ( $\left.\times 10^{\wedge} 4\right)$ and equivalent isotropic displacement parameters $\left(A^{\wedge} 2 \times 10^{\wedge} 3\right)$ for $3 a$.

$\mathrm{U}(\mathrm{eq})$ is defined as one third of the trace of the orthogonalized Uij tensor.

\begin{tabular}{ccccc}
\hline $\mathrm{x}$ & $\mathrm{y}$ & $\mathrm{z}$ & $\mathrm{U}(\mathrm{eq})$ & \\
& & & & \\
\hline $\mathrm{F}(001)$ & $3653(1)$ & $5015(2)$ & $3576(1)$ & $60(1)$ \\
$\mathrm{F}(002)$ & $6529(1)$ & $4893(2)$ & $5943(1)$ & $62(1)$
\end{tabular}




\begin{tabular}{|c|c|c|c|c|}
\hline $\mathrm{F}(003)$ & $4054(1)$ & $2656(2)$ & $6111(1)$ & $58(1)$ \\
\hline $\mathrm{F}(004)$ & $4216(1)$ & $3603(2)$ & $5230(1)$ & $66(1)$ \\
\hline $\mathrm{F}(005)$ & $3622(1)$ & $2316(2)$ & $5018(1)$ & $64(1)$ \\
\hline $\mathrm{O}(006)$ & $3531(1)$ & $8388(1)$ & $2843(1)$ & $41(1)$ \\
\hline $\mathrm{O}(007)$ & $3834(1)$ & $2831(2)$ & $8570(1)$ & $45(1)$ \\
\hline $\mathrm{F}(008)$ & $4500(1)$ & $6024(2)$ & $3908(1)$ & $67(1)$ \\
\hline $\mathrm{O}(009)$ & $3387(1)$ & $4774(2)$ & $2142(1)$ & $51(1)$ \\
\hline $\mathrm{O}(00 \mathrm{~A})$ & $4592(1)$ & $4238(1)$ & $7314(1)$ & $39(1)$ \\
\hline $\mathrm{F}(00 \mathrm{~B})$ & $4423(1)$ & $7507(2)$ & $6480(1)$ & $64(1)$ \\
\hline $\mathrm{F}(00 \mathrm{C})$ & $4832(1)$ & $4829(2)$ & $3291(1)$ & $69(1)$ \\
\hline $\mathrm{O}(00 \mathrm{D})$ & $7279(1)$ & $1654(1)$ & $5721(1)$ & $38(1)$ \\
\hline $\mathrm{F}(00 \mathrm{E})$ & $5203(2)$ & $6483(2)$ & $6105(1)$ & $69(1)$ \\
\hline $\mathrm{O}(00 \mathrm{~F})$ & 2593(1) & $2264(2)$ & $6386(1)$ & $48(1)$ \\
\hline $\mathrm{O}(00 \mathrm{G})$ & $3320(1)$ & $5882(1)$ & $6068(1)$ & $41(1)$ \\
\hline $\mathrm{F}(00 \mathrm{H})$ & $6378(1)$ & $5137(2)$ & $4781(1)$ & $73(1)$ \\
\hline $\mathrm{F}(00 \mathrm{I})$ & $5994(1)$ & $3860(2)$ & $5254(2)$ & $74(1)$ \\
\hline $\mathrm{O}(00 \mathrm{~J})$ & $1196(1)$ & $5313(2)$ & $3668(1)$ & $50(1)$ \\
\hline $\mathrm{O}(00 \mathrm{~K})$ & 1719(1) & $2695(2)$ & $5501(1)$ & $57(1)$ \\
\hline $\mathrm{O}(00 \mathrm{~L})$ & 1994(1) & $7203(2)$ & $6859(1)$ & $57(1)$ \\
\hline $\mathrm{O}(00 \mathrm{M})$ & $8012(2)$ & $5261(2)$ & $5743(1)$ & $57(1)$ \\
\hline $\mathrm{O}(00 \mathrm{~N})$ & $4505(1)$ & $7879(2)$ & $7918(1)$ & $55(1)$ \\
\hline $\mathrm{O}(00 \mathrm{O})$ & $2546(1)$ & $2515(2)$ & $8481(1)$ & $60(1)$ \\
\hline $\mathrm{F}(00 \mathrm{P})$ & $5647(2)$ & $7757(2)$ & $6582(1)$ & $72(1)$ \\
\hline $\mathrm{O}(00 \mathrm{Q})$ & $7784(2)$ & $4494(2)$ & $8206(1)$ & $60(1)$ \\
\hline $\mathrm{O}(00 \mathrm{R})$ & 1951(1) & $7208(2)$ & $8101(1)$ & $56(1)$ \\
\hline $\mathrm{O}(00 \mathrm{~S})$ & $4300(2)$ & $5344(2)$ & $1439(1)$ & $64(1)$ \\
\hline $\mathrm{O}(00 \mathrm{~T})$ & $977(2)$ & $4991(2)$ & $2453(1)$ & $67(1)$ \\
\hline $\mathrm{N}(00 \mathrm{U})$ & 3091(1) & $3621(2)$ & 7771(1) & $40(1)$ \\
\hline $\mathrm{N}(00 \mathrm{~V})$ & $4609(1)$ & $7536(2)$ & $2637(1)$ & $42(1)$ \\
\hline $\mathrm{N}(00 \mathrm{~W})$ & $8186(2)$ & $3784(2)$ & $7228(1)$ & $44(1)$ \\
\hline $\mathrm{N}(00 \mathrm{X})$ & $2878(1)$ & $6429(2)$ & $7555(1)$ & $41(1)$ \\
\hline $\mathrm{N}(00 \mathrm{Y})$ & $1880(1)$ & $6051(2)$ & 2861(1) & $44(1)$ \\
\hline $\mathrm{O}(00 \mathrm{Z})$ & $8329(2)$ & $4936(2)$ & $4603(2)$ & $76(1)$ \\
\hline $\mathrm{N}(010)$ & $5683(1)$ & $5124(2)$ & $7371(2)$ & $44(1)$ \\
\hline $\mathrm{C}(011)$ & $4088(2)$ & $5881(2)$ & $8169(1)$ & $34(1)$ \\
\hline $\mathrm{O}(012)$ & $5616(2)$ & $7444(2)$ & $8483(2)$ & $78(1)$ \\
\hline $\mathrm{O}(013)$ & $9015(2)$ & $4736(2)$ & $7880(2)$ & $83(1)$ \\
\hline $\mathrm{C}(014)$ & $7544(2)$ & $3309(2)$ & $5753(1)$ & $35(1)$ \\
\hline
\end{tabular}




\begin{tabular}{|c|c|c|c|c|}
\hline $\mathrm{N}(015)$ & $7147(2)$ & $2536(2)$ & $4680(1)$ & $50(1)$ \\
\hline $\mathrm{C}(016)$ & $2338(2)$ & $2759(2)$ & $5815(2)$ & $40(1)$ \\
\hline $\mathrm{C}(017)$ & $3872(2)$ & $7642(2)$ & $2751(1)$ & $35(1)$ \\
\hline $\mathrm{C}(018)$ & $4522(2)$ & $5904(2)$ & $7449(1)$ & $34(1)$ \\
\hline $\mathrm{C}(019)$ & $2376(2)$ & $4352(2)$ & $6733(1)$ & $36(1)$ \\
\hline $\mathrm{C}(01 \mathrm{~A})$ & $2405(2)$ & $4030(2)$ & $7463(2)$ & $38(1)$ \\
\hline $\mathrm{C}(01 \mathrm{~B})$ & 4929(2) & 4991(2) & $7366(1)$ & $36(1)$ \\
\hline $\mathrm{N}(01 \mathrm{C})$ & 2921(2) & 4980(2) & $5094(1)$ & $43(1)$ \\
\hline $\mathrm{C}(01 \mathrm{D})$ & $3099(2)$ & $2945(2)$ & $8297(2)$ & $43(1)$ \\
\hline $\mathrm{C}(01 \mathrm{E})$ & $3461(2)$ & $6712(2)$ & $2714(1)$ & $35(1)$ \\
\hline $\mathrm{C}(01 \mathrm{~F})$ & $2943(2)$ & $3485(2)$ & $5622(1)$ & $36(1)$ \\
\hline $\mathrm{C}(01 \mathrm{G})$ & $3965(2)$ & $5333(2)$ & $1998(2)$ & $43(1)$ \\
\hline $\mathrm{C}(01 \mathrm{H})$ & $3298(2)$ & $6112(2)$ & $8197(2)$ & $37(1)$ \\
\hline $\mathrm{C}(01 \mathrm{I})$ & $7302(2)$ & $2400(2)$ & $5390(1)$ & $37(1)$ \\
\hline $\mathrm{C}(01 \mathrm{~J})$ & $7948(2)$ & $4802(2)$ & $5115(2)$ & $49(1)$ \\
\hline $\mathrm{C}(01 \mathrm{~K})$ & $1314(2)$ & $5409(3)$ & $2948(2)$ & $48(1)$ \\
\hline $\mathrm{C}(01 \mathrm{~L})$ & $7334(2)$ & $4045(2)$ & $5148(2)$ & $41(1)$ \\
\hline $\mathrm{C}(01 \mathrm{M})$ & $3053(2)$ & $4244(2)$ & $6238(1)$ & $34(1)$ \\
\hline $\mathrm{C}(01 \mathrm{~N})$ & $3716(2)$ & $3009(2)$ & $5505(2)$ & $47(1)$ \\
\hline $\mathrm{C}(01 \mathrm{O})$ & $4136(2)$ & $5996(2)$ & $2640(2)$ & $38(1)$ \\
\hline $\mathrm{C}(01 \mathrm{P})$ & $8396(2)$ & $4386(3)$ & $7787(2)$ & $56(1)$ \\
\hline $\mathrm{C}(01 \mathrm{Q})$ & $2945(2)$ & $5991(2)$ & $8864(2)$ & $46(1)$ \\
\hline $\mathrm{C}(01 \mathrm{R})$ & $5163(2)$ & $6644(2)$ & $7385(2)$ & $40(1)$ \\
\hline $\mathrm{C}(01 \mathrm{~S})$ & $3027(2)$ & $7083(2)$ & $1413(2)$ & $46(1)$ \\
\hline $\mathrm{C}(01 \mathrm{~T})$ & $6560(2)$ & $4486(2)$ & $5289(2)$ & $51(1)$ \\
\hline $\mathrm{C}(01 \mathrm{U})$ & $8398(2)$ & $3242(2)$ & $5988(2)$ & $39(1)$ \\
\hline $\mathrm{C}(01 \mathrm{~V})$ & $2846(2)$ & $6722(2)$ & $2092(2)$ & $38(1)$ \\
\hline $\mathrm{C}(01 \mathrm{~W})$ & $9698(2)$ & $2838(3)$ & $5659(2)$ & $59(1)$ \\
\hline $\mathrm{C}(01 \mathrm{X})$ & $4274(2)$ & $5462(2)$ & $3357(2)$ & $50(1)$ \\
\hline $\mathrm{C}(01 \mathrm{Y})$ & $3128(2)$ & $5125(2)$ & $5796(1)$ & $37(1)$ \\
\hline $\mathrm{C}(01 Z)$ & $1117(2)$ & $4598(3)$ & $7612(2)$ & $51(1)$ \\
\hline $\mathrm{C}(020)$ & $2097(2)$ & $6400(2)$ & $2178(2)$ & $42(1)$ \\
\hline $\mathrm{C}(021)$ & $5136(2)$ & $7377(2)$ & $7995(2)$ & $47(1)$ \\
\hline $\mathrm{C}(022)$ & $1709(2)$ & $4807(2)$ & $6473(2)$ & $44(1)$ \\
\hline $\mathrm{C}(023)$ & $4494(2)$ & $5584(2)$ & $8798(2)$ & $44(1)$ \\
\hline $\mathrm{C}(024)$ & $8682(2)$ & $3467(2)$ & $6692(2)$ & $41(1)$ \\
\hline $\mathrm{C}(025)$ & $8918(2)$ & 2926(2) & $5476(2)$ & $49(1)$ \\
\hline $\mathrm{C}(026)$ & $476(2)$ & $4874(2)$ & $3889(2)$ & $44(1)$ \\
\hline
\end{tabular}




\begin{tabular}{|c|c|c|c|c|}
\hline $\mathrm{C}(027)$ & $9474(2)$ & $3355(3)$ & $6880(2)$ & $55(1)$ \\
\hline $\mathrm{C}(028)$ & 1081(2) & 4924(3) & $6895(2)$ & $52(1)$ \\
\hline $\mathrm{C}(029)$ & $3369(2)$ & $5705(2)$ & $9486(2)$ & $52(1)$ \\
\hline $\mathrm{C}(02 \mathrm{~A})$ & $2243(2)$ & $6963(2)$ & $7461(2)$ & $44(1)$ \\
\hline $\mathrm{C}(02 \mathrm{~B})$ & $5099(2)$ & $7107(2)$ & $6635(2)$ & $51(1)$ \\
\hline $\mathrm{C}(02 \mathrm{C})$ & $1775(2)$ & $4157(2)$ & $7890(2)$ & $46(1)$ \\
\hline $\mathrm{C}(02 \mathrm{D})$ & $498(2)$ & $5051(3)$ & $4713(2)$ & $57(1)$ \\
\hline $\mathrm{C}(02 \mathrm{E})$ & $1551(2)$ & $6461(3)$ & $1587(2)$ & $59(1)$ \\
\hline $\mathrm{C}(02 \mathrm{~F})$ & $9968(2)$ & $3054(3)$ & $6355(3)$ & $63(1)$ \\
\hline $\mathrm{C}(02 \mathrm{G})$ & $7256(2)$ & $3480(2)$ & $4426(2)$ & $53(1)$ \\
\hline $\mathrm{C}(02 \mathrm{H})$ & $4144(2)$ & $5508(2)$ & $9466(2)$ & $51(1)$ \\
\hline $\mathrm{C}(02 \mathrm{I})$ & $3964(2)$ & 2393(3) & $9299(2)$ & $50(1)$ \\
\hline $\mathrm{C}(02 \mathrm{~J})$ & $-215(2)$ & $5356(3)$ & $3521(2)$ & $58(1)$ \\
\hline $\mathrm{C}(02 \mathrm{~K})$ & $4865(2)$ & $6598(2)$ & $2523(2)$ & $48(1)$ \\
\hline $\mathrm{C}(02 \mathrm{~L})$ & $2694(2)$ & $4027(2)$ & $4920(2)$ & $45(1)$ \\
\hline $\mathrm{C}(02 \mathrm{M})$ & $1754(2)$ & $6805(3)$ & $918(2)$ & $65(1)$ \\
\hline $\mathrm{C}(02 \mathrm{~N})$ & $5930(2)$ & $6078(2)$ & $7453(2)$ & $52(1)$ \\
\hline $\mathrm{C}(02 \mathrm{O})$ & $2489(2)$ & $7119(3)$ & $823(2)$ & $57(1)$ \\
\hline $\mathrm{C}(02 \mathrm{P})$ & $4812(2)$ & 2593(3) & $9473(2)$ & $65(1)$ \\
\hline $\mathrm{C}(02 \mathrm{Q})$ & $2070(2)$ & $1576(3)$ & $6669(2)$ & $62(1)$ \\
\hline $\mathrm{C}(02 \mathrm{R})$ & $3468(3)$ & $2853(4)$ & $9856(2)$ & $71(1)$ \\
\hline $\mathrm{C}(02 \mathrm{~S})$ & $492(2)$ & $3849(3)$ & $3735(2)$ & $62(1)$ \\
\hline $\mathrm{C}(02 \mathrm{~T})$ & $3816(2)$ & $1367(3)$ & $9244(2)$ & $63(1)$ \\
\hline $\mathrm{C}(02 \mathrm{U})$ & $3086(3)$ & $4195(3)$ & $1537(2)$ & $69(1)$ \\
\hline $\mathrm{C}(02 \mathrm{~V})$ & 7861(3) & $5020(3)$ & $8894(2)$ & $74(1)$ \\
\hline $\mathrm{C}(02 \mathrm{~W})$ & $1235(2)$ & $7761(3)$ & $8117(2)$ & $63(1)$ \\
\hline $\mathrm{C}(02 \mathrm{X})$ & $568(2)$ & $7226(4)$ & 7733(3) & $80(1)$ \\
\hline $\mathrm{C}(02 \mathrm{Y})$ & $4360(3)$ & $8536(4)$ & $8491(3)$ & $85(2)$ \\
\hline $\mathrm{C}(02 Z)$ & $1116(4)$ & $7809(6)$ & $8934(3)$ & $116(3)$ \\
\hline $\mathrm{C}(030)$ & $2555(4)$ & $854(3)$ & $7053(3)$ & $87(1)$ \\
\hline $\mathrm{C}(031)$ & $8624(3)$ & $5951(3)$ & $5827(3)$ & $87(2)$ \\
\hline $\mathrm{C}(032)$ & 1349(3) & $8696(3)$ & $7787(3)$ & $88(1)$ \\
\hline $\mathrm{C}(033)$ & $8014(4)$ & $6035(3)$ & $8730(3)$ & $95(2)$ \\
\hline $\mathrm{C}(034)$ & $8493(4)$ & $4591(4)$ & $9392(3)$ & $105(2)$ \\
\hline $\mathrm{C}(035)$ & $7080(4)$ & $4876(4)$ & $9212(2)$ & $96(2)$ \\
\hline $\mathrm{C}(036)$ & $2535(3)$ & $3536(4)$ & $1848(4)$ & $104(2)$ \\
\hline $\mathrm{C}(037)$ & $8500(7)$ & $6469(6)$ & 6493(4) & $176(5)$ \\
\hline $\mathrm{C}(2)$ & $3635(5)$ & $8865(8)$ & $8428(7)$ & $194(5)$ \\
\hline
\end{tabular}


Table 3. Bond lengths $[\mathrm{A}]$ and angles $[\mathrm{deg}]$ for $3 \mathrm{a}$.

\begin{tabular}{|c|c|}
\hline $\mathrm{F}(001)-\mathrm{C}(01 \mathrm{X})$ & $1.332(4)$ \\
\hline $\mathrm{F}(002)-\mathrm{C}(01 \mathrm{~T})$ & $1.331(4)$ \\
\hline $\mathrm{F}(003)-\mathrm{C}(01 \mathrm{~N})$ & $1.326(4)$ \\
\hline $\mathrm{F}(004)-\mathrm{C}(01 \mathrm{~N})$ & $1.335(4)$ \\
\hline $\mathrm{F}(005)-\mathrm{C}(01 \mathrm{~N})$ & $1.342(4)$ \\
\hline $\mathrm{O}(006)-\mathrm{C}(017)$ & $1.245(3)$ \\
\hline $\mathrm{O}(007)-\mathrm{C}(01 \mathrm{D})$ & $1.355(4)$ \\
\hline $\mathrm{O}(007)-\mathrm{C}(02 \mathrm{I})$ & $1.478(3)$ \\
\hline $\mathrm{F}(008)-\mathrm{C}(01 \mathrm{X})$ & $1.334(4)$ \\
\hline $\mathrm{O}(009)-\mathrm{C}(01 \mathrm{G})$ & $1.323(4)$ \\
\hline $\mathrm{O}(009)-\mathrm{C}(02 \mathrm{U})$ & $1.460(4)$ \\
\hline $\mathrm{O}(00 \mathrm{~A})-\mathrm{C}(01 \mathrm{~B})$ & $1.237(3)$ \\
\hline $\mathrm{F}(00 \mathrm{~B})-\mathrm{C}(02 \mathrm{~B})$ & $1.324(4)$ \\
\hline $\mathrm{F}(00 \mathrm{C})-\mathrm{C}(01 \mathrm{X})$ & $1.341(4)$ \\
\hline $\mathrm{O}(00 \mathrm{D})-\mathrm{C}(01 \mathrm{I})$ & $1.237(4)$ \\
\hline $\mathrm{F}(00 \mathrm{E})-\mathrm{C}(02 \mathrm{~B})$ & $1.341(4)$ \\
\hline $\mathrm{O}(00 \mathrm{~F})-\mathrm{C}(016)$ & $1.320(4)$ \\
\hline $\mathrm{O}(00 \mathrm{~F})-\mathrm{C}(02 \mathrm{Q})$ & $1.456(4)$ \\
\hline $\mathrm{O}(00 \mathrm{G})-\mathrm{C}(01 \mathrm{Y})$ & $1.239(4)$ \\
\hline $\mathrm{F}(00 \mathrm{H})-\mathrm{C}(01 \mathrm{~T})$ & $1.345(4)$ \\
\hline $\mathrm{F}(00 \mathrm{I})-\mathrm{C}(01 \mathrm{~T})$ & $1.334(4)$ \\
\hline $\mathrm{O}(00 \mathrm{~J})-\mathrm{C}(01 \mathrm{~K})$ & $1.343(4)$ \\
\hline $\mathrm{O}(00 \mathrm{~J})-\mathrm{C}(026)$ & $1.474(3)$ \\
\hline $\mathrm{O}(00 \mathrm{~K})-\mathrm{C}(016)$ & $1.194(4)$ \\
\hline $\mathrm{O}(00 \mathrm{~L})-\mathrm{C}(02 \mathrm{~A})$ & $1.209(4)$ \\
\hline $\mathrm{O}(00 \mathrm{M})-\mathrm{C}(01 \mathrm{~J})$ & $1.322(4)$ \\
\hline $\mathrm{O}(00 \mathrm{M})-\mathrm{C}(031)$ & $1.458(5)$ \\
\hline $\mathrm{O}(00 \mathrm{~N})-\mathrm{C}(021)$ & $1.315(4)$ \\
\hline $\mathrm{O}(00 \mathrm{~N})-\mathrm{C}(02 \mathrm{Y})$ & $1.443(5)$ \\
\hline $\mathrm{O}(00 \mathrm{O})-\mathrm{C}(01 \mathrm{D})$ & $1.205(4)$ \\
\hline $\mathrm{F}(00 \mathrm{P})-\mathrm{C}(02 \mathrm{~B})$ & $1.343(4)$ \\
\hline $\mathrm{O}(00 \mathrm{Q})-\mathrm{C}(01 \mathrm{P})$ & $1.347(5)$ \\
\hline $\mathrm{O}(00 \mathrm{Q})-\mathrm{C}(02 \mathrm{~V})$ & $1.466(4)$ \\
\hline $\mathrm{O}(00 \mathrm{R})-\mathrm{C}(02 \mathrm{~A})$ & $1.341(4)$ \\
\hline $\mathrm{O}(00 \mathrm{R})-\mathrm{C}(02 \mathrm{~W})$ & $1.478(4)$ \\
\hline
\end{tabular}




\begin{tabular}{|c|c|}
\hline $\mathrm{O}(00 \mathrm{~S})-\mathrm{C}(01 \mathrm{G})$ & $1.198(4)$ \\
\hline $\mathrm{O}(00 \mathrm{~T})-\mathrm{C}(01 \mathrm{~K})$ & $1.210(4)$ \\
\hline $\mathrm{N}(00 \mathrm{U})-\mathrm{H}(00 \mathrm{U})$ & 0.8600 \\
\hline $\mathrm{N}(00 \mathrm{U})-\mathrm{C}(01 \mathrm{~A})$ & $1.419(4)$ \\
\hline $\mathrm{N}(00 \mathrm{U})-\mathrm{C}(01 \mathrm{D})$ & $1.368(4)$ \\
\hline $\mathrm{N}(00 \mathrm{~V})-\mathrm{H}(00 \mathrm{~V})$ & 0.8600 \\
\hline $\mathrm{N}(00 \mathrm{~V})-\mathrm{C}(017)$ & $1.312(4)$ \\
\hline $\mathrm{N}(00 \mathrm{~V})-\mathrm{C}(02 \mathrm{~K})$ & $1.444(4)$ \\
\hline $\mathrm{N}(00 \mathrm{~W})-\mathrm{H}(00 \mathrm{~W})$ & 0.8600 \\
\hline $\mathrm{N}(00 \mathrm{~W})-\mathrm{C}(01 \mathrm{P})$ & $1.374(4)$ \\
\hline $\mathrm{N}(00 \mathrm{~W})-\mathrm{C}(024)$ & $1.410(4)$ \\
\hline $\mathrm{N}(00 \mathrm{X})-\mathrm{H}(00 \mathrm{X})$ & 0.8600 \\
\hline $\mathrm{N}(00 \mathrm{X})-\mathrm{C}(01 \mathrm{H})$ & $1.422(4)$ \\
\hline $\mathrm{N}(00 \mathrm{X})-\mathrm{C}(02 \mathrm{~A})$ & $1.349(4)$ \\
\hline $\mathrm{N}(00 \mathrm{Y})-\mathrm{H}(00 \mathrm{Y})$ & 0.8600 \\
\hline $\mathrm{N}(00 \mathrm{Y})-\mathrm{C}(01 \mathrm{~K})$ & $1.365(4)$ \\
\hline $\mathrm{N}(00 \mathrm{Y})-\mathrm{C}(020)$ & $1.410(4)$ \\
\hline $\mathrm{O}(00 \mathrm{Z})-\mathrm{C}(01 \mathrm{~J})$ & $1.186(4)$ \\
\hline $\mathrm{N}(010)-\mathrm{H}(010)$ & 0.8600 \\
\hline $\mathrm{N}(010)-\mathrm{C}(01 \mathrm{~B})$ & $1.321(4)$ \\
\hline $\mathrm{N}(010)-\mathrm{C}(02 \mathrm{~N})$ & $1.449(4)$ \\
\hline $\mathrm{C}(011)-\mathrm{C}(018)$ & $1.543(4)$ \\
\hline $\mathrm{C}(011)-\mathrm{C}(01 \mathrm{H})$ & $1.413(4)$ \\
\hline $\mathrm{C}(011)-\mathrm{C}(023)$ & $1.383(4)$ \\
\hline $\mathrm{O}(012)-\mathrm{C}(021)$ & $1.190(4)$ \\
\hline $\mathrm{O}(013)-\mathrm{C}(01 \mathrm{P})$ & $1.191(5)$ \\
\hline $\mathrm{C}(014)-\mathrm{H}(014)$ & 0.9800 \\
\hline $\mathrm{C}(014)-\mathrm{C}(01 \mathrm{I})$ & $1.521(4)$ \\
\hline $\mathrm{C}(014)-\mathrm{C}(01 \mathrm{~L})$ & $1.561(4)$ \\
\hline $\mathrm{C}(014)-\mathrm{C}(01 \mathrm{U})$ & $1.524(4)$ \\
\hline $\mathrm{N}(015)-\mathrm{H}(015)$ & 0.8600 \\
\hline $\mathrm{N}(015)-\mathrm{C}(01 \mathrm{I})$ & $1.322(4)$ \\
\hline $\mathrm{N}(015)-\mathrm{C}(02 \mathrm{G})$ & $1.455(4)$ \\
\hline $\mathrm{C}(016)-\mathrm{C}(01 \mathrm{~F})$ & $1.538(4)$ \\
\hline $\mathrm{C}(017)-\mathrm{C}(01 \mathrm{E})$ & $1.521(4)$ \\
\hline $\mathrm{C}(018)-\mathrm{H}(018)$ & 0.9800 \\
\hline $\mathrm{C}(018)-\mathrm{C}(01 \mathrm{~B})$ & $1.508(4)$ \\
\hline $\mathrm{C}(018)-\mathrm{C}(01 \mathrm{R})$ & $1.551(4)$ \\
\hline
\end{tabular}




\begin{tabular}{|c|c|}
\hline $\mathrm{C}(019)-\mathrm{C}(01 \mathrm{~A})$ & $1.406(4)$ \\
\hline $\mathrm{C}(019)-\mathrm{C}(01 \mathrm{M})$ & $1.523(4)$ \\
\hline $\mathrm{C}(019)-\mathrm{C}(022)$ & $1.392(4)$ \\
\hline $\mathrm{C}(01 \mathrm{~A})-\mathrm{C}(02 \mathrm{C})$ & $1.384(4)$ \\
\hline $\mathrm{N}(01 \mathrm{C})-\mathrm{H}(01 \mathrm{C})$ & 0.8600 \\
\hline $\mathrm{N}(01 \mathrm{C})-\mathrm{C}(01 \mathrm{Y})$ & $1.327(4)$ \\
\hline $\mathrm{N}(01 \mathrm{C})-\mathrm{C}(02 \mathrm{~L})$ & $1.463(4)$ \\
\hline $\mathrm{C}(01 \mathrm{E})-\mathrm{H}(01 \mathrm{E})$ & 0.9800 \\
\hline $\mathrm{C}(01 \mathrm{E})-\mathrm{C}(01 \mathrm{O})$ & $1.574(4)$ \\
\hline$C(01 E)-C(01 V)$ & $1.517(4)$ \\
\hline $\mathrm{C}(01 \mathrm{~F})-\mathrm{C}(01 \mathrm{M})$ & $1.573(4)$ \\
\hline $\mathrm{C}(01 \mathrm{~F})-\mathrm{C}(01 \mathrm{~N})$ & $1.531(4)$ \\
\hline $\mathrm{C}(01 \mathrm{~F})-\mathrm{C}(02 \mathrm{~L})$ & $1.542(4)$ \\
\hline $\mathrm{C}(01 \mathrm{G})-\mathrm{C}(01 \mathrm{O})$ & $1.529(4)$ \\
\hline $\mathrm{C}(01 \mathrm{H})-\mathrm{C}(01 \mathrm{Q})$ & $1.399(4)$ \\
\hline $\mathrm{C}(01 \mathrm{~J})-\mathrm{C}(01 \mathrm{~L})$ & $1.530(4)$ \\
\hline $\mathrm{C}(01 \mathrm{~L})-\mathrm{C}(01 \mathrm{~T})$ & $1.519(4)$ \\
\hline $\mathrm{C}(01 \mathrm{~L})-\mathrm{C}(02 \mathrm{G})$ & $1.547(4)$ \\
\hline $\mathrm{C}(01 \mathrm{M})-\mathrm{H}(01 \mathrm{M})$ & 0.9800 \\
\hline $\mathrm{C}(01 \mathrm{M})-\mathrm{C}(01 \mathrm{Y})$ & $1.516(4)$ \\
\hline $\mathrm{C}(01 \mathrm{O})-\mathrm{C}(01 \mathrm{X})$ & $1.527(4)$ \\
\hline $\mathrm{C}(01 \mathrm{O})-\mathrm{C}(02 \mathrm{~K})$ & $1.558(4)$ \\
\hline $\mathrm{C}(01 \mathrm{Q})-\mathrm{H}(01 \mathrm{Q})$ & 0.9300 \\
\hline $\mathrm{C}(01 \mathrm{Q})-\mathrm{C}(029)$ & $1.381(5)$ \\
\hline $\mathrm{C}(01 \mathrm{R})-\mathrm{C}(021)$ & $1.537(4)$ \\
\hline $\mathrm{C}(01 \mathrm{R})-\mathrm{C}(02 \mathrm{~B})$ & $1.519(4)$ \\
\hline $\mathrm{C}(01 \mathrm{R})-\mathrm{C}(02 \mathrm{~N})$ & $1.561(4)$ \\
\hline $\mathrm{C}(01 \mathrm{~S})-\mathrm{H}(01 \mathrm{~S})$ & 0.9300 \\
\hline $\mathrm{C}(01 \mathrm{~S})-\mathrm{C}(01 \mathrm{~V})$ & 1.391(4) \\
\hline $\mathrm{C}(01 \mathrm{~S})-\mathrm{C}(02 \mathrm{O})$ & $1.389(5)$ \\
\hline $\mathrm{C}(01 \mathrm{U})-\mathrm{C}(024)$ & $1.389(4)$ \\
\hline $\mathrm{C}(01 \mathrm{U})-\mathrm{C}(025)$ & $1.405(4)$ \\
\hline $\mathrm{C}(01 \mathrm{~V})-\mathrm{C}(020)$ & $1.397(4)$ \\
\hline $\mathrm{C}(01 \mathrm{~W})-\mathrm{H}(01 \mathrm{~W})$ & 0.9300 \\
\hline $\mathrm{C}(01 \mathrm{~W})-\mathrm{C}(025)$ & $1.382(5)$ \\
\hline $\mathrm{C}(01 \mathrm{~W})-\mathrm{C}(02 \mathrm{~F})$ & $1.365(6)$ \\
\hline $\mathrm{C}(01 \mathrm{Z})-\mathrm{H}(01 \mathrm{Z})$ & 0.9300 \\
\hline $\mathrm{C}(01 \mathrm{Z})-\mathrm{C}(028)$ & $1.385(5)$ \\
\hline
\end{tabular}




\begin{tabular}{|c|c|}
\hline $\mathrm{C}(01 \mathrm{Z})-\mathrm{C}(02 \mathrm{C})$ & $1.382(5)$ \\
\hline $\mathrm{C}(020)-\mathrm{C}(02 \mathrm{E})$ & $1.401(4)$ \\
\hline $\mathrm{C}(022)-\mathrm{H}(022)$ & 0.9300 \\
\hline $\mathrm{C}(022)-\mathrm{C}(028)$ & $1.375(5)$ \\
\hline $\mathrm{C}(023)-\mathrm{H}(023)$ & 0.9300 \\
\hline $\mathrm{C}(023)-\mathrm{C}(02 \mathrm{H})$ & $1.391(5)$ \\
\hline $\mathrm{C}(024)-\mathrm{C}(027)$ & $1.409(4)$ \\
\hline $\mathrm{C}(025)-\mathrm{H}(025)$ & 0.9300 \\
\hline $\mathrm{C}(026)-\mathrm{C}(02 \mathrm{D})$ & $1.521(5)$ \\
\hline $\mathrm{C}(026)-\mathrm{C}(02 \mathrm{~J})$ & $1.512(5)$ \\
\hline $\mathrm{C}(026)-\mathrm{C}(02 \mathrm{~S})$ & $1.508(5)$ \\
\hline $\mathrm{C}(027)-\mathrm{H}(027)$ & 0.9300 \\
\hline $\mathrm{C}(027)-\mathrm{C}(02 \mathrm{~F})$ & $1.384(6)$ \\
\hline $\mathrm{C}(028)-\mathrm{H}(028)$ & 0.9300 \\
\hline $\mathrm{C}(029)-\mathrm{H}(029)$ & 0.9300 \\
\hline $\mathrm{C}(029)-\mathrm{C}(02 \mathrm{H})$ & $1.376(5)$ \\
\hline $\mathrm{C}(02 \mathrm{C})-\mathrm{H}(02 \mathrm{~T})$ & 0.9300 \\
\hline $\mathrm{C}(02 \mathrm{D})-\mathrm{H}(02 \mathrm{~A})$ & 0.9600 \\
\hline $\mathrm{C}(02 \mathrm{D})-\mathrm{H}(02 \mathrm{~B})$ & 0.9600 \\
\hline $\mathrm{C}(02 \mathrm{D})-\mathrm{H}(02 \mathrm{C})$ & 0.9600 \\
\hline $\mathrm{C}(02 \mathrm{E})-\mathrm{H}(02 \mathrm{E})$ & 0.9300 \\
\hline $\mathrm{C}(02 \mathrm{E})-\mathrm{C}(02 \mathrm{M})$ & $1.378(6)$ \\
\hline $\mathrm{C}(02 \mathrm{~F})-\mathrm{H}(02 \mathrm{Q})$ & 0.9300 \\
\hline $\mathrm{C}(02 \mathrm{G})-\mathrm{H}(02 \mathrm{R})$ & 0.9700 \\
\hline $\mathrm{C}(02 \mathrm{G})-\mathrm{H}(02 \mathrm{~S})$ & 0.9700 \\
\hline $\mathrm{C}(02 \mathrm{H})-\mathrm{H}(1 \mathrm{AA})$ & 0.9300 \\
\hline $\mathrm{C}(02 \mathrm{I})-\mathrm{C}(02 \mathrm{P})$ & $1.516(5)$ \\
\hline $\mathrm{C}(02 \mathrm{I})-\mathrm{C}(02 \mathrm{R})$ & $1.515(5)$ \\
\hline $\mathrm{C}(02 \mathrm{I})-\mathrm{C}(02 \mathrm{~T})$ & $1.506(6)$ \\
\hline $\mathrm{C}(02 \mathrm{~J})-\mathrm{H}(02 \mathrm{D})$ & 0.9600 \\
\hline $\mathrm{C}(02 \mathrm{~J})-\mathrm{H}(02 \mathrm{~F})$ & 0.9600 \\
\hline $\mathrm{C}(02 \mathrm{~J})-\mathrm{H}(02 \mathrm{G})$ & 0.9600 \\
\hline $\mathrm{C}(02 \mathrm{~K})-\mathrm{H}(02 \mathrm{H})$ & 0.9700 \\
\hline $\mathrm{C}(02 \mathrm{~K})-\mathrm{H}(02 \mathrm{I})$ & 0.9700 \\
\hline $\mathrm{C}(02 \mathrm{~L})-\mathrm{H}(02 \mathrm{U})$ & 0.9700 \\
\hline $\mathrm{C}(02 \mathrm{~L})-\mathrm{H}(02 \mathrm{~V})$ & 0.9700 \\
\hline $\mathrm{C}(02 \mathrm{M})-\mathrm{H}(02 \mathrm{M})$ & 0.9300 \\
\hline $\mathrm{C}(02 \mathrm{M})-\mathrm{C}(02 \mathrm{O})$ & $1.372(6)$ \\
\hline
\end{tabular}




\begin{tabular}{|c|c|}
\hline $\mathrm{C}(02 \mathrm{~N})-\mathrm{H}(2 \mathrm{AA})$ & 0.9700 \\
\hline $\mathrm{C}(02 \mathrm{~N})-\mathrm{H}$ & 0.9700 \\
\hline $\mathrm{C}(02 \mathrm{O})-\mathrm{H}(02 \mathrm{O})$ & 0.9300 \\
\hline $\mathrm{C}(02 \mathrm{P})-\mathrm{H}(02 \mathrm{~W})$ & 0.9600 \\
\hline $\mathrm{C}(02 \mathrm{P})-\mathrm{H}(02 \mathrm{X})$ & 0.9600 \\
\hline $\mathrm{C}(02 \mathrm{P})-\mathrm{H}(02 \mathrm{Y})$ & 0.9600 \\
\hline $\mathrm{C}(02 \mathrm{Q})-\mathrm{H}(02 \mathrm{Z})$ & 0.9700 \\
\hline $\mathrm{C}(02 \mathrm{Q})-\mathrm{HA}$ & 0.9700 \\
\hline $\mathrm{C}(02 \mathrm{Q})-\mathrm{C}(030)$ & $1.492(7)$ \\
\hline $\mathrm{C}(02 \mathrm{R})-\mathrm{H}(02)$ & 0.9600 \\
\hline $\mathrm{C}(02 \mathrm{R})-\mathrm{HB}$ & 0.9600 \\
\hline $\mathrm{C}(02 \mathrm{R})-\mathrm{HC}$ & 0.9600 \\
\hline $\mathrm{C}(02 \mathrm{~S})-\mathrm{H}(02 \mathrm{~J})$ & 0.9600 \\
\hline $\mathrm{C}(02 \mathrm{~S})-\mathrm{H}(02 \mathrm{~K})$ & 0.9600 \\
\hline $\mathrm{C}(02 \mathrm{~S})-\mathrm{H}(02 \mathrm{~L})$ & 0.9600 \\
\hline $\mathrm{C}(02 \mathrm{~T})-\mathrm{H}(0 \mathrm{AA})$ & 0.9600 \\
\hline $\mathrm{C}(02 \mathrm{~T})-\mathrm{HD}$ & 0.9600 \\
\hline $\mathrm{C}(02 \mathrm{~T})-\mathrm{HE}$ & 0.9600 \\
\hline $\mathrm{C}(02 \mathrm{U})-\mathrm{H}(02 \mathrm{~N})$ & 0.9700 \\
\hline $\mathrm{C}(02 \mathrm{U})-\mathrm{H}(02 \mathrm{P})$ & 0.9700 \\
\hline $\mathrm{C}(02 \mathrm{U})-\mathrm{C}(036)$ & $1.481(8)$ \\
\hline $\mathrm{C}(02 \mathrm{~V})-\mathrm{C}(033)$ & $1.523(7)$ \\
\hline $\mathrm{C}(02 \mathrm{~V})-\mathrm{C}(034)$ & $1.519(7)$ \\
\hline $\mathrm{C}(02 \mathrm{~V})-\mathrm{C}(035)$ & $1.513(8)$ \\
\hline $\mathrm{C}(02 \mathrm{~W})-\mathrm{C}(02 \mathrm{X})$ & $1.532(7)$ \\
\hline $\mathrm{C}(02 \mathrm{~W})-\mathrm{C}(02 \mathrm{Z})$ & $1.513(6)$ \\
\hline $\mathrm{C}(02 \mathrm{~W})-\mathrm{C}(032)$ & $1.497(7)$ \\
\hline $\mathrm{C}(02 \mathrm{X})-\mathrm{H}(3 \mathrm{AA})$ & 0.9600 \\
\hline $\mathrm{C}(02 \mathrm{X})-\mathrm{HF}$ & 0.9600 \\
\hline $\mathrm{C}(02 \mathrm{X})-\mathrm{HG}$ & 0.9600 \\
\hline $\mathrm{C}(02 \mathrm{Y})-\mathrm{H}(4 \mathrm{AA})$ & 0.9300 \\
\hline $\mathrm{C}(02 \mathrm{Y})-\mathrm{C}(2)$ & $1.343(9)$ \\
\hline $\mathrm{C}(02 \mathrm{Z})-\mathrm{H}(5 \mathrm{AA})$ & 0.9600 \\
\hline $\mathrm{C}(02 \mathrm{Z})-\mathrm{HH}$ & 0.9600 \\
\hline $\mathrm{C}(02 \mathrm{Z})-\mathrm{HI}$ & 0.9600 \\
\hline $\mathrm{C}(030)-\mathrm{H}(03 \mathrm{R})$ & 0.9600 \\
\hline $\mathrm{C}(030)-\mathrm{H}(03 \mathrm{~S})$ & 0.9600 \\
\hline $\mathrm{C}(030)-\mathrm{H}(03 \mathrm{~T})$ & 0.9600 \\
\hline
\end{tabular}




\begin{tabular}{|c|c|}
\hline $\mathrm{C}(031)-\mathrm{H}(03 \mathrm{D})$ & 0.9700 \\
\hline $\mathrm{C}(031)-\mathrm{H}(03 \mathrm{E})$ & 0.9700 \\
\hline $\mathrm{C}(031)-\mathrm{C}(037)$ & $1.451(10$ \\
\hline $\mathrm{C}(032)-\mathrm{H}(03 \mathrm{U})$ & 0.9600 \\
\hline $\mathrm{C}(032)-\mathrm{H}(03 \mathrm{~V})$ & 0.9600 \\
\hline $\mathrm{C}(032)-\mathrm{H}(03 \mathrm{~W})$ & 0.9600 \\
\hline $\mathrm{C}(033)-\mathrm{H}(03 \mathrm{~F})$ & 0.9600 \\
\hline $\mathrm{C}(033)-\mathrm{H}(03 \mathrm{G})$ & 0.9600 \\
\hline $\mathrm{C}(033)-\mathrm{H}(03 \mathrm{H})$ & 0.9600 \\
\hline $\mathrm{C}(034)-\mathrm{H}(03 \mathrm{I})$ & 0.9600 \\
\hline $\mathrm{C}(034)-\mathrm{H}(03 \mathrm{~J})$ & 0.9600 \\
\hline $\mathrm{C}(034)-\mathrm{H}(03 \mathrm{~K})$ & 0.9600 \\
\hline $\mathrm{C}(035)-\mathrm{H}(03 \mathrm{~L})$ & 0.9600 \\
\hline $\mathrm{C}(035)-\mathrm{H}(03 \mathrm{M})$ & 0.9600 \\
\hline $\mathrm{C}(035)-\mathrm{H}(03 \mathrm{~N})$ & 0.9600 \\
\hline $\mathrm{C}(036)-\mathrm{H}(03 \mathrm{~A})$ & 0.9600 \\
\hline $\mathrm{C}(036)-\mathrm{H}(03 \mathrm{~B})$ & 0.9600 \\
\hline $\mathrm{C}(036)-\mathrm{H}(03 \mathrm{C})$ & 0.9600 \\
\hline $\mathrm{C}(037)-\mathrm{H}(03 \mathrm{O})$ & 0.9600 \\
\hline $\mathrm{C}(037)-\mathrm{H}(03 \mathrm{P})$ & 0.9600 \\
\hline $\mathrm{C}(037)-\mathrm{H}(03 \mathrm{Q})$ & 0.9600 \\
\hline $\mathrm{C}(2)-\mathrm{H}(2 \mathrm{~A})$ & 0.9600 \\
\hline $\mathrm{C}(2)-\mathrm{H}(2 \mathrm{~B})$ & 0.9600 \\
\hline $\mathrm{C}(2)-\mathrm{H}(2 \mathrm{C})$ & 0.9600 \\
\hline $\mathrm{C}(01 \mathrm{D})-\mathrm{O}(007)-\mathrm{C}(02 \mathrm{I})$ & $118.8(2)$ \\
\hline $\mathrm{C}(01 \mathrm{G})-\mathrm{O}(009)-\mathrm{C}(02 \mathrm{U})$ & $116.5(3)$ \\
\hline $\mathrm{C}(016)-\mathrm{O}(00 \mathrm{~F})-\mathrm{C}(02 \mathrm{Q})$ & $117.3(3)$ \\
\hline $\mathrm{C}(01 \mathrm{~K})-\mathrm{O}(00 \mathrm{~J})-\mathrm{C}(026)$ & $118.9(2)$ \\
\hline $\mathrm{C}(01 \mathrm{~J})-\mathrm{O}(00 \mathrm{M})-\mathrm{C}(031)$ & $117.7(3)$ \\
\hline $\mathrm{C}(021)-\mathrm{O}(00 \mathrm{~N})-\mathrm{C}(02 \mathrm{Y})$ & $117.4(3)$ \\
\hline $\mathrm{C}(01 \mathrm{P})-\mathrm{O}(00 \mathrm{Q})-\mathrm{C}(02 \mathrm{~V})$ & $120.2(3)$ \\
\hline $\mathrm{C}(02 \mathrm{~A})-\mathrm{O}(00 \mathrm{R})-\mathrm{C}(02 \mathrm{~W})$ & $120.9(2)$ \\
\hline $\mathrm{C}(01 \mathrm{~A})-\mathrm{N}(00 \mathrm{U})-\mathrm{H}(00 \mathrm{U})$ & 118.2 \\
\hline $\mathrm{C}(01 \mathrm{D})-\mathrm{N}(00 \mathrm{U})-\mathrm{H}(00 \mathrm{U})$ & 118.2 \\
\hline $\mathrm{C}(01 \mathrm{D})-\mathrm{N}(00 \mathrm{U})-\mathrm{C}(01 \mathrm{~A})$ & $123.6(2)$ \\
\hline $\mathrm{C}(017)-\mathrm{N}(00 \mathrm{~V})-\mathrm{H}(00 \mathrm{~V})$ & 121.9 \\
\hline $\mathrm{C}(017)-\mathrm{N}(00 \mathrm{~V})-\mathrm{C}(02 \mathrm{~K})$ & $116.2(2)$ \\
\hline
\end{tabular}




\begin{tabular}{|c|c|}
\hline $\mathrm{C}(02 \mathrm{~K})-\mathrm{N}(00 \mathrm{~V})-\mathrm{H}(00 \mathrm{~V})$ & 121.9 \\
\hline $\mathrm{C}(01 \mathrm{P})-\mathrm{N}(00 \mathrm{~W})-\mathrm{H}(00 \mathrm{~W})$ & 117.7 \\
\hline $\mathrm{C}(01 \mathrm{P})-\mathrm{N}(00 \mathrm{~W})-\mathrm{C}(024)$ & $124.7(3)$ \\
\hline $\mathrm{C}(024)-\mathrm{N}(00 \mathrm{~W})-\mathrm{H}(00 \mathrm{~W})$ & 117.7 \\
\hline $\mathrm{C}(01 \mathrm{H})-\mathrm{N}(00 \mathrm{X})-\mathrm{H}(00 \mathrm{X})$ & 113.9 \\
\hline $\mathrm{C}(02 \mathrm{~A})-\mathrm{N}(00 \mathrm{X})-\mathrm{H}(00 \mathrm{X})$ & 113.9 \\
\hline $\mathrm{C}(02 \mathrm{~A})-\mathrm{N}(00 \mathrm{X})-\mathrm{C}(01 \mathrm{H})$ & $132.2(2)$ \\
\hline $\mathrm{C}(01 \mathrm{~K})-\mathrm{N}(00 \mathrm{Y})-\mathrm{H}(00 \mathrm{Y})$ & 117.7 \\
\hline $\mathrm{C}(01 \mathrm{~K})-\mathrm{N}(00 \mathrm{Y})-\mathrm{C}(020)$ & $124.7(2)$ \\
\hline $\mathrm{C}(020)-\mathrm{N}(00 \mathrm{Y})-\mathrm{H}(00 \mathrm{Y})$ & 117.7 \\
\hline $\mathrm{C}(01 \mathrm{~B})-\mathrm{N}(010)-\mathrm{H}(010)$ & 122.4 \\
\hline $\mathrm{C}(01 \mathrm{~B})-\mathrm{N}(010)-\mathrm{C}(02 \mathrm{~N})$ & $115.3(2)$ \\
\hline $\mathrm{C}(02 \mathrm{~N})-\mathrm{N}(010)-\mathrm{H}(010)$ & 122.4 \\
\hline $\mathrm{C}(01 \mathrm{H})-\mathrm{C}(011)-\mathrm{C}(018)$ & $122.7(2)$ \\
\hline $\mathrm{C}(023)-\mathrm{C}(011)-\mathrm{C}(018)$ & $117.4(2)$ \\
\hline $\mathrm{C}(023)-\mathrm{C}(011)-\mathrm{C}(01 \mathrm{H})$ & $119.9(3)$ \\
\hline $\mathrm{C}(01 \mathrm{I})-\mathrm{C}(014)-\mathrm{H}(014)$ & 109.8 \\
\hline $\mathrm{C}(01 \mathrm{I})-\mathrm{C}(014)-\mathrm{C}(01 \mathrm{~L})$ & $103.4(2)$ \\
\hline $\mathrm{C}(01 \mathrm{I})-\mathrm{C}(014)-\mathrm{C}(01 \mathrm{U})$ & $108.1(2)$ \\
\hline $\mathrm{C}(01 \mathrm{~L})-\mathrm{C}(014)-\mathrm{H}(014)$ & 109.8 \\
\hline $\mathrm{C}(01 \mathrm{U})-\mathrm{C}(014)-\mathrm{H}(014)$ & 109.8 \\
\hline $\mathrm{C}(01 \mathrm{U})-\mathrm{C}(014)-\mathrm{C}(01 \mathrm{~L})$ & $115.6(2)$ \\
\hline $\mathrm{C}(01 \mathrm{I})-\mathrm{N}(015)-\mathrm{H}(015)$ & 122.4 \\
\hline $\mathrm{C}(01 \mathrm{I})-\mathrm{N}(015)-\mathrm{C}(02 \mathrm{G})$ & $115.2(3)$ \\
\hline $\mathrm{C}(02 \mathrm{G})-\mathrm{N}(015)-\mathrm{H}(015)$ & 122.4 \\
\hline $\mathrm{O}(00 \mathrm{~F})-\mathrm{C}(016)-\mathrm{C}(01 \mathrm{~F})$ & $110.1(2)$ \\
\hline $\mathrm{O}(00 \mathrm{~K})-\mathrm{C}(016)-\mathrm{O}(00 \mathrm{~F})$ & $126.4(3)$ \\
\hline $\mathrm{O}(00 \mathrm{~K})-\mathrm{C}(016)-\mathrm{C}(01 \mathrm{~F})$ & $123.4(3)$ \\
\hline $\mathrm{O}(006)-\mathrm{C}(017)-\mathrm{N}(00 \mathrm{~V})$ & $126.5(2)$ \\
\hline $\mathrm{O}(006)-\mathrm{C}(017)-\mathrm{C}(01 \mathrm{E})$ & $123.0(2)$ \\
\hline $\mathrm{N}(00 \mathrm{~V})-\mathrm{C}(017)-\mathrm{C}(01 \mathrm{E})$ & $110.4(2)$ \\
\hline $\mathrm{C}(011)-\mathrm{C}(018)-\mathrm{H}(018)$ & 108.6 \\
\hline $\mathrm{C}(011)-\mathrm{C}(018)-\mathrm{C}(01 \mathrm{R})$ & $117.4(2)$ \\
\hline $\mathrm{C}(01 \mathrm{~B})-\mathrm{C}(018)-\mathrm{C}(011)$ & $108.5(2)$ \\
\hline $\mathrm{C}(01 \mathrm{~B})-\mathrm{C}(018)-\mathrm{H}(018)$ & 108.6 \\
\hline $\mathrm{C}(01 \mathrm{~B})-\mathrm{C}(018)-\mathrm{C}(01 \mathrm{R})$ & $104.8(2)$ \\
\hline $\mathrm{C}(01 \mathrm{R})-\mathrm{C}(018)-\mathrm{H}(018)$ & 108.6 \\
\hline $\mathrm{C}(01 \mathrm{~A})-\mathrm{C}(019)-\mathrm{C}(01 \mathrm{M})$ & $122.2(2)$ \\
\hline
\end{tabular}




\begin{tabular}{|c|c|}
\hline $\mathrm{C}(022)-\mathrm{C}(019)-\mathrm{C}(01 \mathrm{~A})$ & $117.9(3)$ \\
\hline $\mathrm{C}(022)-\mathrm{C}(019)-\mathrm{C}(01 \mathrm{M})$ & $119.9(2)$ \\
\hline $\mathrm{C}(019)-\mathrm{C}(01 \mathrm{~A})-\mathrm{N}(00 \mathrm{U})$ & $120.1(2)$ \\
\hline $\mathrm{C}(02 \mathrm{C})-\mathrm{C}(01 \mathrm{~A})-\mathrm{N}(00 \mathrm{U})$ & $120.1(3)$ \\
\hline $\mathrm{C}(02 \mathrm{C})-\mathrm{C}(01 \mathrm{~A})-\mathrm{C}(019)$ & $119.7(3)$ \\
\hline $\mathrm{O}(00 \mathrm{~A})-\mathrm{C}(01 \mathrm{~B})-\mathrm{N}(010)$ & $126.4(3)$ \\
\hline $\mathrm{O}(00 \mathrm{~A})-\mathrm{C}(01 \mathrm{~B})-\mathrm{C}(018)$ & $123.8(2)$ \\
\hline $\mathrm{N}(010)-\mathrm{C}(01 \mathrm{~B})-\mathrm{C}(018)$ & $109.9(2)$ \\
\hline $\mathrm{C}(01 \mathrm{Y})-\mathrm{N}(01 \mathrm{C})-\mathrm{H}(01 \mathrm{C})$ & 122.8 \\
\hline $\mathrm{C}(01 \mathrm{Y})-\mathrm{N}(01 \mathrm{C})-\mathrm{C}(02 \mathrm{~L})$ & $114.4(2)$ \\
\hline $\mathrm{C}(02 \mathrm{~L})-\mathrm{N}(01 \mathrm{C})-\mathrm{H}(01 \mathrm{C})$ & 122.8 \\
\hline $\mathrm{O}(007)-\mathrm{C}(01 \mathrm{D})-\mathrm{N}(00 \mathrm{U})$ & $108.7(2)$ \\
\hline $\mathrm{O}(00 \mathrm{O})-\mathrm{C}(01 \mathrm{D})-\mathrm{O}(007)$ & $125.6(3)$ \\
\hline $\mathrm{O}(00 \mathrm{O})-\mathrm{C}(01 \mathrm{D})-\mathrm{N}(00 \mathrm{U})$ & $125.7(3)$ \\
\hline $\mathrm{C}(017)-\mathrm{C}(01 \mathrm{E})-\mathrm{H}(01 \mathrm{E})$ & 109.1 \\
\hline$C(017)-C(01 E)-C(01 O)$ & $103.6(2)$ \\
\hline $\mathrm{C}(01 \mathrm{O})-\mathrm{C}(01 \mathrm{E})-\mathrm{H}(01 \mathrm{E})$ & 109.1 \\
\hline $\mathrm{C}(01 \mathrm{~V})-\mathrm{C}(01 \mathrm{E})-\mathrm{C}(017)$ & $109.5(2)$ \\
\hline $\mathrm{C}(01 \mathrm{~V})-\mathrm{C}(01 \mathrm{E})-\mathrm{H}(01 \mathrm{E})$ & 109.1 \\
\hline $\mathrm{C}(01 \mathrm{~V})-\mathrm{C}(01 \mathrm{E})-\mathrm{C}(01 \mathrm{O})$ & $116.1(2)$ \\
\hline $\mathrm{C}(016)-\mathrm{C}(01 \mathrm{~F})-\mathrm{C}(01 \mathrm{M})$ & $111.8(2)$ \\
\hline $\mathrm{C}(016)-\mathrm{C}(01 \mathrm{~F})-\mathrm{C}(02 \mathrm{~L})$ & $111.7(2)$ \\
\hline $\mathrm{C}(01 \mathrm{~N})-\mathrm{C}(01 \mathrm{~F})-\mathrm{C}(016)$ & $109.7(2)$ \\
\hline $\mathrm{C}(01 \mathrm{~N})-\mathrm{C}(01 \mathrm{~F})-\mathrm{C}(01 \mathrm{M})$ & $109.7(2)$ \\
\hline $\mathrm{C}(01 \mathrm{~N})-\mathrm{C}(01 \mathrm{~F})-\mathrm{C}(02 \mathrm{~L})$ & $108.9(2)$ \\
\hline $\mathrm{C}(02 \mathrm{~L})-\mathrm{C}(01 \mathrm{~F})-\mathrm{C}(01 \mathrm{M})$ & $104.8(2)$ \\
\hline $\mathrm{O}(009)-\mathrm{C}(01 \mathrm{G})-\mathrm{C}(01 \mathrm{O})$ & $110.6(2)$ \\
\hline $\mathrm{O}(00 \mathrm{~S})-\mathrm{C}(01 \mathrm{G})-\mathrm{O}(009)$ & $125.5(3)$ \\
\hline $\mathrm{O}(00 \mathrm{~S})-\mathrm{C}(01 \mathrm{G})-\mathrm{C}(01 \mathrm{O})$ & $123.9(3)$ \\
\hline $\mathrm{C}(011)-\mathrm{C}(01 \mathrm{H})-\mathrm{N}(00 \mathrm{X})$ & $120.3(2)$ \\
\hline $\mathrm{C}(01 \mathrm{Q})-\mathrm{C}(01 \mathrm{H})-\mathrm{N}(00 \mathrm{X})$ & $121.7(3)$ \\
\hline $\mathrm{C}(01 \mathrm{Q})-\mathrm{C}(01 \mathrm{H})-\mathrm{C}(011)$ & $118.0(3)$ \\
\hline $\mathrm{O}(00 \mathrm{D})-\mathrm{C}(01 \mathrm{I})-\mathrm{C}(014)$ & $123.7(2)$ \\
\hline $\mathrm{O}(00 \mathrm{D})-\mathrm{C}(01 \mathrm{I})-\mathrm{N}(015)$ & $126.7(3)$ \\
\hline $\mathrm{N}(015)-\mathrm{C}(01 \mathrm{I})-\mathrm{C}(014)$ & $109.6(2)$ \\
\hline $\mathrm{O}(00 \mathrm{M})-\mathrm{C}(01 \mathrm{~J})-\mathrm{C}(01 \mathrm{~L})$ & $110.8(2)$ \\
\hline $\mathrm{O}(00 \mathrm{Z})-\mathrm{C}(01 \mathrm{~J})-\mathrm{O}(00 \mathrm{M})$ & $124.8(3)$ \\
\hline $\mathrm{O}(00 \mathrm{Z})-\mathrm{C}(01 \mathrm{~J})-\mathrm{C}(01 \mathrm{~L})$ & $124.3(3)$ \\
\hline
\end{tabular}




\begin{tabular}{|c|c|}
\hline $\mathrm{O}(00 \mathrm{~J})-\mathrm{C}(01 \mathrm{~K})-\mathrm{N}(00 \mathrm{Y})$ & 109.2(2) \\
\hline $\mathrm{O}(00 \mathrm{~T})-\mathrm{C}(01 \mathrm{~K})-\mathrm{O}(00 \mathrm{~J})$ & $125.7(3)$ \\
\hline $\mathrm{O}(00 \mathrm{~T})-\mathrm{C}(01 \mathrm{~K})-\mathrm{N}(00 \mathrm{Y})$ & $125.2(3)$ \\
\hline $\mathrm{C}(01 \mathrm{~J})-\mathrm{C}(01 \mathrm{~L})-\mathrm{C}(014)$ & $112.0(2)$ \\
\hline $\mathrm{C}(01 \mathrm{~J})-\mathrm{C}(01 \mathrm{~L})-\mathrm{C}(02 \mathrm{G})$ & $112.1(3)$ \\
\hline $\mathrm{C}(01 \mathrm{~T})-\mathrm{C}(01 \mathrm{~L})-\mathrm{C}(014)$ & $110.1(2)$ \\
\hline $\mathrm{C}(01 \mathrm{~T})-\mathrm{C}(01 \mathrm{~L})-\mathrm{C}(01 \mathrm{~J})$ & 109.2(3) \\
\hline $\mathrm{C}(01 \mathrm{~T})-\mathrm{C}(01 \mathrm{~L})-\mathrm{C}(02 \mathrm{G})$ & $109.0(3)$ \\
\hline $\mathrm{C}(02 \mathrm{G})-\mathrm{C}(01 \mathrm{~L})-\mathrm{C}(014)$ & $104.3(2)$ \\
\hline $\mathrm{C}(019)-\mathrm{C}(01 \mathrm{M})-\mathrm{C}(01 \mathrm{~F})$ & $115.1(2)$ \\
\hline $\mathrm{C}(019)-\mathrm{C}(01 \mathrm{M})-\mathrm{H}(01 \mathrm{M})$ & 110.2 \\
\hline $\mathrm{C}(01 \mathrm{~F})-\mathrm{C}(01 \mathrm{M})-\mathrm{H}(01 \mathrm{M})$ & 110.2 \\
\hline $\mathrm{C}(01 \mathrm{Y})-\mathrm{C}(01 \mathrm{M})-\mathrm{C}(019)$ & $108.5(2)$ \\
\hline $\mathrm{C}(01 \mathrm{Y})-\mathrm{C}(01 \mathrm{M})-\mathrm{C}(01 \mathrm{~F})$ & $102.5(2)$ \\
\hline $\mathrm{C}(01 \mathrm{Y})-\mathrm{C}(01 \mathrm{M})-\mathrm{H}(01 \mathrm{M})$ & 110.2 \\
\hline $\mathrm{F}(003)-\mathrm{C}(01 \mathrm{~N})-\mathrm{F}(004)$ & $106.9(3)$ \\
\hline $\mathrm{F}(003)-\mathrm{C}(01 \mathrm{~N})-\mathrm{F}(005)$ & 107.3(3) \\
\hline $\mathrm{F}(003)-\mathrm{C}(01 \mathrm{~N})-\mathrm{C}(01 \mathrm{~F})$ & $114.2(2)$ \\
\hline $\mathrm{F}(004)-\mathrm{C}(01 \mathrm{~N})-\mathrm{F}(005)$ & 107.1(3) \\
\hline $\mathrm{F}(004)-\mathrm{C}(01 \mathrm{~N})-\mathrm{C}(01 \mathrm{~F})$ & $110.6(3)$ \\
\hline $\mathrm{F}(005)-\mathrm{C}(01 \mathrm{~N})-\mathrm{C}(01 \mathrm{~F})$ & $110.4(3)$ \\
\hline $\mathrm{C}(01 \mathrm{G})-\mathrm{C}(01 \mathrm{O})-\mathrm{C}(01 \mathrm{E})$ & $111.2(2)$ \\
\hline $\mathrm{C}(01 \mathrm{G})-\mathrm{C}(01 \mathrm{O})-\mathrm{C}(02 \mathrm{~K})$ & $111.9(2)$ \\
\hline $\mathrm{C}(01 \mathrm{X})-\mathrm{C}(01 \mathrm{O})-\mathrm{C}(01 \mathrm{E})$ & $110.1(2)$ \\
\hline $\mathrm{C}(01 \mathrm{X})-\mathrm{C}(01 \mathrm{O})-\mathrm{C}(01 \mathrm{G})$ & $110.6(2)$ \\
\hline $\mathrm{C}(01 \mathrm{X})-\mathrm{C}(01 \mathrm{O})-\mathrm{C}(02 \mathrm{~K})$ & $107.8(3)$ \\
\hline $\mathrm{C}(02 \mathrm{~K})-\mathrm{C}(01 \mathrm{O})-\mathrm{C}(01 \mathrm{E})$ & $104.9(2)$ \\
\hline $\mathrm{O}(00 \mathrm{Q})-\mathrm{C}(01 \mathrm{P})-\mathrm{N}(00 \mathrm{~W})$ & $107.8(3)$ \\
\hline $\mathrm{O}(013)-\mathrm{C}(01 \mathrm{P})-\mathrm{O}(00 \mathrm{Q})$ & $126.9(3)$ \\
\hline $\mathrm{O}(013)-\mathrm{C}(01 \mathrm{P})-\mathrm{N}(00 \mathrm{~W})$ & $125.4(4)$ \\
\hline $\mathrm{C}(01 \mathrm{H})-\mathrm{C}(01 \mathrm{Q})-\mathrm{H}(01 \mathrm{Q})$ & 119.7 \\
\hline $\mathrm{C}(029)-\mathrm{C}(01 \mathrm{Q})-\mathrm{C}(01 \mathrm{H})$ & $120.7(3)$ \\
\hline $\mathrm{C}(029)-\mathrm{C}(01 \mathrm{Q})-\mathrm{H}(01 \mathrm{Q})$ & 119.7 \\
\hline $\mathrm{C}(018)-\mathrm{C}(01 \mathrm{R})-\mathrm{C}(02 \mathrm{~N})$ & $104.1(2)$ \\
\hline $\mathrm{C}(021)-\mathrm{C}(01 \mathrm{R})-\mathrm{C}(018)$ & $111.9(2)$ \\
\hline $\mathrm{C}(021)-\mathrm{C}(01 \mathrm{R})-\mathrm{C}(02 \mathrm{~N})$ & 111.1(2) \\
\hline $\mathrm{C}(02 \mathrm{~B})-\mathrm{C}(01 \mathrm{R})-\mathrm{C}(018)$ & $110.6(2)$ \\
\hline $\mathrm{C}(02 \mathrm{~B})-\mathrm{C}(01 \mathrm{R})-\mathrm{C}(021)$ & $110.1(3)$ \\
\hline
\end{tabular}




\begin{tabular}{|c|c|}
\hline $\mathrm{C}(02 \mathrm{~B})-\mathrm{C}(01 \mathrm{R})-\mathrm{C}(02 \mathrm{~N})$ & $109.0(3)$ \\
\hline $\mathrm{C}(01 \mathrm{~V})-\mathrm{C}(01 \mathrm{~S})-\mathrm{H}(01 \mathrm{~S})$ & 119.1 \\
\hline $\mathrm{C}(02 \mathrm{O})-\mathrm{C}(01 \mathrm{~S})-\mathrm{H}(01 \mathrm{~S})$ & 119.1 \\
\hline $\mathrm{C}(02 \mathrm{O})-\mathrm{C}(01 \mathrm{~S})-\mathrm{C}(01 \mathrm{~V})$ & $121.9(3)$ \\
\hline $\mathrm{F}(002)-\mathrm{C}(01 \mathrm{~T})-\mathrm{F}(00 \mathrm{H})$ & $106.7(3)$ \\
\hline $\mathrm{F}(002)-\mathrm{C}(01 \mathrm{~T})-\mathrm{F}(00 \mathrm{I})$ & $106.2(3)$ \\
\hline $\mathrm{F}(002)-\mathrm{C}(01 \mathrm{~T})-\mathrm{C}(01 \mathrm{~L})$ & $114.4(3)$ \\
\hline $\mathrm{F}(00 \mathrm{H})-\mathrm{C}(01 \mathrm{~T})-\mathrm{C}(01 \mathrm{~L})$ & $110.8(3)$ \\
\hline $\mathrm{F}(00 \mathrm{I})-\mathrm{C}(01 \mathrm{~T})-\mathrm{F}(00 \mathrm{H})$ & $107.1(3)$ \\
\hline $\mathrm{F}(00 \mathrm{I})-\mathrm{C}(01 \mathrm{~T})-\mathrm{C}(01 \mathrm{~L})$ & $111.2(3)$ \\
\hline $\mathrm{C}(024)-\mathrm{C}(01 \mathrm{U})-\mathrm{C}(014)$ & $122.6(2)$ \\
\hline $\mathrm{C}(024)-\mathrm{C}(01 \mathrm{U})-\mathrm{C}(025)$ & $118.6(3)$ \\
\hline $\mathrm{C}(025)-\mathrm{C}(01 \mathrm{U})-\mathrm{C}(014)$ & $118.7(3)$ \\
\hline $\mathrm{C}(01 \mathrm{~S})-\mathrm{C}(01 \mathrm{~V})-\mathrm{C}(01 \mathrm{E})$ & 119.1(3) \\
\hline $\mathrm{C}(01 \mathrm{~S})-\mathrm{C}(01 \mathrm{~V})-\mathrm{C}(020)$ & $118.5(3)$ \\
\hline $\mathrm{C}(020)-\mathrm{C}(01 \mathrm{~V})-\mathrm{C}(01 \mathrm{E})$ & $122.3(2)$ \\
\hline $\mathrm{C}(025)-\mathrm{C}(01 \mathrm{~W})-\mathrm{H}(01 \mathrm{~W})$ & 120.3 \\
\hline $\mathrm{C}(02 \mathrm{~F})-\mathrm{C}(01 \mathrm{~W})-\mathrm{H}(01 \mathrm{~W})$ & 120.3 \\
\hline $\mathrm{C}(02 \mathrm{~F})-\mathrm{C}(01 \mathrm{~W})-\mathrm{C}(025)$ & 119.3(3) \\
\hline $\mathrm{F}(001)-\mathrm{C}(01 \mathrm{X})-\mathrm{F}(008)$ & $106.4(3)$ \\
\hline $\mathrm{F}(001)-\mathrm{C}(01 \mathrm{X})-\mathrm{F}(00 \mathrm{C})$ & 107.1(3) \\
\hline $\mathrm{F}(001)-\mathrm{C}(01 \mathrm{X})-\mathrm{C}(01 \mathrm{O})$ & $114.0(3)$ \\
\hline $\mathrm{F}(008)-\mathrm{C}(01 \mathrm{X})-\mathrm{F}(00 \mathrm{C})$ & $107.3(3)$ \\
\hline $\mathrm{F}(008)-\mathrm{C}(01 \mathrm{X})-\mathrm{C}(01 \mathrm{O})$ & $111.2(3)$ \\
\hline $\mathrm{F}(00 \mathrm{C})-\mathrm{C}(01 \mathrm{X})-\mathrm{C}(01 \mathrm{O})$ & $110.5(3)$ \\
\hline $\mathrm{O}(00 \mathrm{G})-\mathrm{C}(01 \mathrm{Y})-\mathrm{N}(01 \mathrm{C})$ & $125.3(3)$ \\
\hline $\mathrm{O}(00 \mathrm{G})-\mathrm{C}(01 \mathrm{Y})-\mathrm{C}(01 \mathrm{M})$ & $123.9(2)$ \\
\hline $\mathrm{N}(01 \mathrm{C})-\mathrm{C}(01 \mathrm{Y})-\mathrm{C}(01 \mathrm{M})$ & $110.7(2)$ \\
\hline $\mathrm{C}(028)-\mathrm{C}(01 \mathrm{Z})-\mathrm{H}(01 \mathrm{Z})$ & 120.0 \\
\hline $\mathrm{C}(02 \mathrm{C})-\mathrm{C}(01 \mathrm{Z})-\mathrm{H}(01 \mathrm{Z})$ & 120.0 \\
\hline $\mathrm{C}(02 \mathrm{C})-\mathrm{C}(01 \mathrm{Z})-\mathrm{C}(028)$ & $120.0(3)$ \\
\hline $\mathrm{C}(01 \mathrm{~V})-\mathrm{C}(020)-\mathrm{N}(00 \mathrm{Y})$ & $120.6(3)$ \\
\hline$C(01 V)-C(020)-C(02 E)$ & $119.3(3)$ \\
\hline $\mathrm{C}(02 \mathrm{E})-\mathrm{C}(020)-\mathrm{N}(00 \mathrm{Y})$ & $120.1(3)$ \\
\hline $\mathrm{O}(00 \mathrm{~N})-\mathrm{C}(021)-\mathrm{C}(01 \mathrm{R})$ & $110.9(2)$ \\
\hline $\mathrm{O}(012)-\mathrm{C}(021)-\mathrm{O}(00 \mathrm{~N})$ & $125.6(3)$ \\
\hline $\mathrm{O}(012)-\mathrm{C}(021)-\mathrm{C}(01 \mathrm{R})$ & $123.4(3)$ \\
\hline $\mathrm{C}(019)-\mathrm{C}(022)-\mathrm{H}(022)$ & 118.8 \\
\hline
\end{tabular}




\begin{tabular}{|c|c|}
\hline $\mathrm{C}(028)-\mathrm{C}(022)-\mathrm{C}(019)$ & $122.4(3)$ \\
\hline $\mathrm{C}(028)-\mathrm{C}(022)-\mathrm{H}(022)$ & 118.8 \\
\hline $\mathrm{C}(011)-\mathrm{C}(023)-\mathrm{H}(023)$ & 119.3 \\
\hline $\mathrm{C}(011)-\mathrm{C}(023)-\mathrm{C}(02 \mathrm{H})$ & $121.5(3)$ \\
\hline $\mathrm{C}(02 \mathrm{H})-\mathrm{C}(023)-\mathrm{H}(023)$ & 119.3 \\
\hline $\mathrm{C}(01 \mathrm{U})-\mathrm{C}(024)-\mathrm{N}(00 \mathrm{~W})$ & $120.9(3)$ \\
\hline $\mathrm{C}(01 \mathrm{U})-\mathrm{C}(024)-\mathrm{C}(027)$ & $119.6(3)$ \\
\hline $\mathrm{C}(027)-\mathrm{C}(024)-\mathrm{N}(00 \mathrm{~W})$ & $119.4(3)$ \\
\hline $\mathrm{C}(01 \mathrm{U})-\mathrm{C}(025)-\mathrm{H}(025)$ & 119.3 \\
\hline $\mathrm{C}(01 \mathrm{~W})-\mathrm{C}(025)-\mathrm{C}(01 \mathrm{U})$ & $121.4(3)$ \\
\hline $\mathrm{C}(01 \mathrm{~W})-\mathrm{C}(025)-\mathrm{H}(025)$ & 119.3 \\
\hline $\mathrm{O}(00 \mathrm{~J})-\mathrm{C}(026)-\mathrm{C}(02 \mathrm{D})$ & $102.5(2)$ \\
\hline $\mathrm{O}(00 \mathrm{~J})-\mathrm{C}(026)-\mathrm{C}(02 \mathrm{~J})$ & $110.1(3)$ \\
\hline $\mathrm{O}(00 \mathrm{~J})-\mathrm{C}(026)-\mathrm{C}(02 \mathrm{~S})$ & $110.4(3)$ \\
\hline $\mathrm{C}(02 \mathrm{~J})-\mathrm{C}(026)-\mathrm{C}(02 \mathrm{D})$ & $109.9(3)$ \\
\hline $\mathrm{C}(02 \mathrm{~S})-\mathrm{C}(026)-\mathrm{C}(02 \mathrm{D})$ & $110.4(3)$ \\
\hline $\mathrm{C}(02 \mathrm{~S})-\mathrm{C}(026)-\mathrm{C}(02 \mathrm{~J})$ & $113.0(3)$ \\
\hline $\mathrm{C}(024)-\mathrm{C}(027)-\mathrm{H}(027)$ & 120.1 \\
\hline $\mathrm{C}(02 \mathrm{~F})-\mathrm{C}(027)-\mathrm{C}(024)$ & $119.8(3)$ \\
\hline $\mathrm{C}(02 \mathrm{~F})-\mathrm{C}(027)-\mathrm{H}(027)$ & 120.1 \\
\hline $\mathrm{C}(01 \mathrm{Z})-\mathrm{C}(028)-\mathrm{H}(028)$ & 120.5 \\
\hline $\mathrm{C}(022)-\mathrm{C}(028)-\mathrm{C}(01 \mathrm{Z})$ & $119.0(3)$ \\
\hline $\mathrm{C}(022)-\mathrm{C}(028)-\mathrm{H}(028)$ & 120.5 \\
\hline $\mathrm{C}(01 \mathrm{Q})-\mathrm{C}(029)-\mathrm{H}(029)$ & 119.3 \\
\hline $\mathrm{C}(02 \mathrm{H})-\mathrm{C}(029)-\mathrm{C}(01 \mathrm{Q})$ & $121.4(3)$ \\
\hline $\mathrm{C}(02 \mathrm{H})-\mathrm{C}(029)-\mathrm{H}(029)$ & 119.3 \\
\hline $\mathrm{O}(00 \mathrm{~L})-\mathrm{C}(02 \mathrm{~A})-\mathrm{O}(00 \mathrm{R})$ & $125.4(3)$ \\
\hline $\mathrm{O}(00 \mathrm{~L})-\mathrm{C}(02 \mathrm{~A})-\mathrm{N}(00 \mathrm{X})$ & $122.1(3)$ \\
\hline $\mathrm{O}(00 \mathrm{R})-\mathrm{C}(02 \mathrm{~A})-\mathrm{N}(00 \mathrm{X})$ & $112.4(2)$ \\
\hline $\mathrm{F}(00 \mathrm{~B})-\mathrm{C}(02 \mathrm{~B})-\mathrm{F}(00 \mathrm{E})$ & $106.8(3)$ \\
\hline $\mathrm{F}(00 \mathrm{~B})-\mathrm{C}(02 \mathrm{~B})-\mathrm{F}(00 \mathrm{P})$ & $107.5(3)$ \\
\hline $\mathrm{F}(00 \mathrm{~B})-\mathrm{C}(02 \mathrm{~B})-\mathrm{C}(01 \mathrm{R})$ & $114.1(3)$ \\
\hline $\mathrm{F}(00 \mathrm{E})-\mathrm{C}(02 \mathrm{~B})-\mathrm{F}(00 \mathrm{P})$ & $107.3(3)$ \\
\hline $\mathrm{F}(00 \mathrm{E})-\mathrm{C}(02 \mathrm{~B})-\mathrm{C}(01 \mathrm{R})$ & $110.2(3)$ \\
\hline $\mathrm{F}(00 \mathrm{P})-\mathrm{C}(02 \mathrm{~B})-\mathrm{C}(01 \mathrm{R})$ & $110.6(3)$ \\
\hline $\mathrm{C}(01 \mathrm{~A})-\mathrm{C}(02 \mathrm{C})-\mathrm{H}(02 \mathrm{~T})$ & 119.5 \\
\hline $\mathrm{C}(01 \mathrm{Z})-\mathrm{C}(02 \mathrm{C})-\mathrm{C}(01 \mathrm{~A})$ & $121.0(3)$ \\
\hline $\mathrm{C}(01 \mathrm{Z})-\mathrm{C}(02 \mathrm{C})-\mathrm{H}(02 \mathrm{~T})$ & 119.5 \\
\hline
\end{tabular}




\begin{tabular}{|c|c|}
\hline $\mathrm{C}(026)-\mathrm{C}(02 \mathrm{D})-\mathrm{H}(02 \mathrm{~A})$ & 109.5 \\
\hline $\mathrm{C}(026)-\mathrm{C}(02 \mathrm{D})-\mathrm{H}(02 \mathrm{~B})$ & 109.5 \\
\hline $\mathrm{C}(026)-\mathrm{C}(02 \mathrm{D})-\mathrm{H}(02 \mathrm{C})$ & 109.5 \\
\hline $\mathrm{H}(02 \mathrm{~A})-\mathrm{C}(02 \mathrm{D})-\mathrm{H}(02 \mathrm{~B})$ & 109.5 \\
\hline $\mathrm{H}(02 \mathrm{~A})-\mathrm{C}(02 \mathrm{D})-\mathrm{H}(02 \mathrm{C})$ & 109.5 \\
\hline $\mathrm{H}(02 \mathrm{~B})-\mathrm{C}(02 \mathrm{D})-\mathrm{H}(02 \mathrm{C})$ & 109.5 \\
\hline $\mathrm{C}(020)-\mathrm{C}(02 \mathrm{E})-\mathrm{H}(02 \mathrm{E})$ & 119.7 \\
\hline $\mathrm{C}(02 \mathrm{M})-\mathrm{C}(02 \mathrm{E})-\mathrm{C}(020)$ & $120.6(3)$ \\
\hline $\mathrm{C}(02 \mathrm{M})-\mathrm{C}(02 \mathrm{E})-\mathrm{H}(02 \mathrm{E})$ & 119.7 \\
\hline $\mathrm{C}(01 \mathrm{~W})-\mathrm{C}(02 \mathrm{~F})-\mathrm{C}(027)$ & $121.2(3)$ \\
\hline $\mathrm{C}(01 \mathrm{~W})-\mathrm{C}(02 \mathrm{~F})-\mathrm{H}(02 \mathrm{Q})$ & 119.4 \\
\hline $\mathrm{C}(027)-\mathrm{C}(02 \mathrm{~F})-\mathrm{H}(02 \mathrm{Q})$ & 119.4 \\
\hline $\mathrm{N}(015)-\mathrm{C}(02 \mathrm{G})-\mathrm{C}(01 \mathrm{~L})$ & $103.4(2)$ \\
\hline $\mathrm{N}(015)-\mathrm{C}(02 \mathrm{G})-\mathrm{H}(02 \mathrm{R})$ & 111.1 \\
\hline $\mathrm{N}(015)-\mathrm{C}(02 \mathrm{G})-\mathrm{H}(02 \mathrm{~S})$ & 111.1 \\
\hline $\mathrm{C}(01 \mathrm{~L})-\mathrm{C}(02 \mathrm{G})-\mathrm{H}(02 \mathrm{R})$ & 111.1 \\
\hline $\mathrm{C}(01 \mathrm{~L})-\mathrm{C}(02 \mathrm{G})-\mathrm{H}(02 \mathrm{~S})$ & 111.1 \\
\hline $\mathrm{H}(02 \mathrm{R})-\mathrm{C}(02 \mathrm{G})-\mathrm{H}(02 \mathrm{~S})$ & 109.0 \\
\hline $\mathrm{C}(023)-\mathrm{C}(02 \mathrm{H})-\mathrm{H}(1 \mathrm{AA})$ & 120.8 \\
\hline $\mathrm{C}(029)-\mathrm{C}(02 \mathrm{H})-\mathrm{C}(023)$ & $118.5(3)$ \\
\hline $\mathrm{C}(029)-\mathrm{C}(02 \mathrm{H})-\mathrm{H}(1 \mathrm{AA})$ & 120.8 \\
\hline $\mathrm{O}(007)-\mathrm{C}(02 \mathrm{I})-\mathrm{C}(02 \mathrm{P})$ & $102.0(3)$ \\
\hline $\mathrm{O}(007)-\mathrm{C}(02 \mathrm{I})-\mathrm{C}(02 \mathrm{R})$ & $110.2(3)$ \\
\hline $\mathrm{O}(007)-\mathrm{C}(02 \mathrm{I})-\mathrm{C}(02 \mathrm{~T})$ & $110.1(3)$ \\
\hline $\mathrm{C}(02 \mathrm{R})-\mathrm{C}(02 \mathrm{I})-\mathrm{C}(02 \mathrm{P})$ & $110.8(3)$ \\
\hline $\mathrm{C}(02 \mathrm{~T})-\mathrm{C}(02 \mathrm{I})-\mathrm{C}(02 \mathrm{P})$ & $111.2(3)$ \\
\hline $\mathrm{C}(02 \mathrm{~T})-\mathrm{C}(02 \mathrm{I})-\mathrm{C}(02 \mathrm{R})$ & $112.1(3)$ \\
\hline $\mathrm{C}(026)-\mathrm{C}(02 \mathrm{~J})-\mathrm{H}(02 \mathrm{D})$ & 109.5 \\
\hline $\mathrm{C}(026)-\mathrm{C}(02 \mathrm{~J})-\mathrm{H}(02 \mathrm{~F})$ & 109.5 \\
\hline $\mathrm{C}(026)-\mathrm{C}(02 \mathrm{~J})-\mathrm{H}(02 \mathrm{G})$ & 109.5 \\
\hline $\mathrm{H}(02 \mathrm{D})-\mathrm{C}(02 \mathrm{~J})-\mathrm{H}(02 \mathrm{~F})$ & 109.5 \\
\hline $\mathrm{H}(02 \mathrm{D})-\mathrm{C}(02 \mathrm{~J})-\mathrm{H}(02 \mathrm{G})$ & 109.5 \\
\hline $\mathrm{H}(02 \mathrm{~F})-\mathrm{C}(02 \mathrm{~J})-\mathrm{H}(02 \mathrm{G})$ & 109.5 \\
\hline $\mathrm{N}(00 \mathrm{~V})-\mathrm{C}(02 \mathrm{~K})-\mathrm{C}(01 \mathrm{O})$ & $104.3(2)$ \\
\hline $\mathrm{N}(00 \mathrm{~V})-\mathrm{C}(02 \mathrm{~K})-\mathrm{H}(02 \mathrm{H})$ & 110.9 \\
\hline $\mathrm{N}(00 \mathrm{~V})-\mathrm{C}(02 \mathrm{~K})-\mathrm{H}(02 \mathrm{I})$ & 110.9 \\
\hline $\mathrm{C}(01 \mathrm{O})-\mathrm{C}(02 \mathrm{~K})-\mathrm{H}(02 \mathrm{H})$ & 110.9 \\
\hline $\mathrm{C}(01 \mathrm{O})-\mathrm{C}(02 \mathrm{~K})-\mathrm{H}(02 \mathrm{I})$ & 110.9 \\
\hline
\end{tabular}




\begin{tabular}{|c|c|}
\hline $\mathrm{H}(02 \mathrm{H})-\mathrm{C}(02 \mathrm{~K})-\mathrm{H}(02 \mathrm{I})$ & 108.9 \\
\hline $\mathrm{N}(01 \mathrm{C})-\mathrm{C}(02 \mathrm{~L})-\mathrm{C}(01 \mathrm{~F})$ & $103.7(2)$ \\
\hline $\mathrm{N}(01 \mathrm{C})-\mathrm{C}(02 \mathrm{~L})-\mathrm{H}(02 \mathrm{U})$ & 111.0 \\
\hline $\mathrm{N}(01 \mathrm{C})-\mathrm{C}(02 \mathrm{~L})-\mathrm{H}(02 \mathrm{~V})$ & 111.0 \\
\hline $\mathrm{C}(01 \mathrm{~F})-\mathrm{C}(02 \mathrm{~L})-\mathrm{H}(02 \mathrm{U})$ & 111.0 \\
\hline $\mathrm{C}(01 \mathrm{~F})-\mathrm{C}(02 \mathrm{~L})-\mathrm{H}(02 \mathrm{~V})$ & 111.0 \\
\hline $\mathrm{H}(02 \mathrm{U})-\mathrm{C}(02 \mathrm{~L})-\mathrm{H}(02 \mathrm{~V})$ & 109.0 \\
\hline $\mathrm{C}(02 \mathrm{E})-\mathrm{C}(02 \mathrm{M})-\mathrm{H}(02 \mathrm{M})$ & 119.6 \\
\hline $\mathrm{C}(02 \mathrm{O})-\mathrm{C}(02 \mathrm{M})-\mathrm{C}(02 \mathrm{E})$ & $120.7(3)$ \\
\hline $\mathrm{C}(02 \mathrm{O})-\mathrm{C}(02 \mathrm{M})-\mathrm{H}(02 \mathrm{M})$ & 119.6 \\
\hline $\mathrm{N}(010)-\mathrm{C}(02 \mathrm{~N})-\mathrm{C}(01 \mathrm{R})$ & 104.1(2) \\
\hline $\mathrm{N}(010)-\mathrm{C}(02 \mathrm{~N})-\mathrm{H}(2 \mathrm{AA})$ & 110.9 \\
\hline $\mathrm{N}(010)-\mathrm{C}(02 \mathrm{~N})-\mathrm{H}$ & 110.9 \\
\hline $\mathrm{C}(01 \mathrm{R})-\mathrm{C}(02 \mathrm{~N})-\mathrm{H}(2 \mathrm{AA})$ & 110.9 \\
\hline $\mathrm{C}(01 \mathrm{R})-\mathrm{C}(02 \mathrm{~N})-\mathrm{H}$ & 110.9 \\
\hline $\mathrm{H}(2 \mathrm{AA})-\mathrm{C}(02 \mathrm{~N})-\mathrm{H}$ & 109.0 \\
\hline $\mathrm{C}(01 \mathrm{~S})-\mathrm{C}(02 \mathrm{O})-\mathrm{H}(02 \mathrm{O})$ & 120.5 \\
\hline $\mathrm{C}(02 \mathrm{M})-\mathrm{C}(02 \mathrm{O})-\mathrm{C}(01 \mathrm{~S})$ & $118.9(3)$ \\
\hline $\mathrm{C}(02 \mathrm{M})-\mathrm{C}(02 \mathrm{O})-\mathrm{H}(02 \mathrm{O})$ & 120.5 \\
\hline $\mathrm{C}(02 \mathrm{I})-\mathrm{C}(02 \mathrm{P})-\mathrm{H}(02 \mathrm{~W})$ & 109.5 \\
\hline $\mathrm{C}(02 \mathrm{I})-\mathrm{C}(02 \mathrm{P})-\mathrm{H}(02 \mathrm{X})$ & 109.5 \\
\hline $\mathrm{C}(02 \mathrm{I})-\mathrm{C}(02 \mathrm{P})-\mathrm{H}(02 \mathrm{Y})$ & 109.5 \\
\hline $\mathrm{H}(02 \mathrm{~W})-\mathrm{C}(02 \mathrm{P})-\mathrm{H}(02 \mathrm{X})$ & 109.5 \\
\hline $\mathrm{H}(02 \mathrm{~W})-\mathrm{C}(02 \mathrm{P})-\mathrm{H}(02 \mathrm{Y})$ & 109.5 \\
\hline $\mathrm{H}(02 \mathrm{X})-\mathrm{C}(02 \mathrm{P})-\mathrm{H}(02 \mathrm{Y})$ & 109.5 \\
\hline $\mathrm{O}(00 \mathrm{~F})-\mathrm{C}(02 \mathrm{Q})-\mathrm{H}(02 \mathrm{Z})$ & 110.3 \\
\hline $\mathrm{O}(00 \mathrm{~F})-\mathrm{C}(02 \mathrm{Q})-\mathrm{HA}$ & 110.3 \\
\hline $\mathrm{O}(00 \mathrm{~F})-\mathrm{C}(02 \mathrm{Q})-\mathrm{C}(030)$ & 107.3(3) \\
\hline $\mathrm{H}(02 \mathrm{Z})-\mathrm{C}(02 \mathrm{Q})-\mathrm{HA}$ & 108.5 \\
\hline $\mathrm{C}(030)-\mathrm{C}(02 \mathrm{Q})-\mathrm{H}(02 \mathrm{Z})$ & 110.3 \\
\hline $\mathrm{C}(030)-\mathrm{C}(02 \mathrm{Q})-\mathrm{HA}$ & 110.3 \\
\hline $\mathrm{C}(02 \mathrm{I})-\mathrm{C}(02 \mathrm{R})-\mathrm{H}(02)$ & 109.5 \\
\hline $\mathrm{C}(02 \mathrm{I})-\mathrm{C}(02 \mathrm{R})-\mathrm{HB}$ & 109.5 \\
\hline $\mathrm{C}(02 \mathrm{I})-\mathrm{C}(02 \mathrm{R})-\mathrm{HC}$ & 109.5 \\
\hline $\mathrm{H}(02)-\mathrm{C}(02 \mathrm{R})-\mathrm{HB}$ & 109.5 \\
\hline $\mathrm{H}(02)-\mathrm{C}(02 \mathrm{R})-\mathrm{HC}$ & 109.5 \\
\hline $\mathrm{HB}-\mathrm{C}(02 \mathrm{R})-\mathrm{HC}$ & 109.5 \\
\hline $\mathrm{C}(026)-\mathrm{C}(02 \mathrm{~S})-\mathrm{H}(02 \mathrm{~J})$ & 109.5 \\
\hline
\end{tabular}




\begin{tabular}{|c|c|}
\hline $\mathrm{C}(026)-\mathrm{C}(02 \mathrm{~S})-\mathrm{H}(02 \mathrm{~K})$ & 109.5 \\
\hline $\mathrm{C}(026)-\mathrm{C}(02 \mathrm{~S})-\mathrm{H}(02 \mathrm{~L})$ & 109.5 \\
\hline $\mathrm{H}(02 \mathrm{~J})-\mathrm{C}(02 \mathrm{~S})-\mathrm{H}(02 \mathrm{~K})$ & 109.5 \\
\hline $\mathrm{H}(02 \mathrm{~J})-\mathrm{C}(02 \mathrm{~S})-\mathrm{H}(02 \mathrm{~L})$ & 109.5 \\
\hline $\mathrm{H}(02 \mathrm{~K})-\mathrm{C}(02 \mathrm{~S})-\mathrm{H}(02 \mathrm{~L})$ & 109.5 \\
\hline $\mathrm{C}(02 \mathrm{I})-\mathrm{C}(02 \mathrm{~T})-\mathrm{H}(0 \mathrm{AA})$ & 109.5 \\
\hline $\mathrm{C}(02 \mathrm{I})-\mathrm{C}(02 \mathrm{~T})-\mathrm{HD}$ & 109.5 \\
\hline $\mathrm{C}(02 \mathrm{I})-\mathrm{C}(02 \mathrm{~T})-\mathrm{HE}$ & 109.5 \\
\hline $\mathrm{H}(0 \mathrm{AA})-\mathrm{C}(02 \mathrm{~T})-\mathrm{HD}$ & 109.5 \\
\hline $\mathrm{H}(0 \mathrm{AA})-\mathrm{C}(02 \mathrm{~T})-\mathrm{HE}$ & 109.5 \\
\hline $\mathrm{HD}-\mathrm{C}(02 \mathrm{~T})-\mathrm{HE}$ & 109.5 \\
\hline $\mathrm{O}(009)-\mathrm{C}(02 \mathrm{U})-\mathrm{H}(02 \mathrm{~N})$ & 110.3 \\
\hline $\mathrm{O}(009)-\mathrm{C}(02 \mathrm{U})-\mathrm{H}(02 \mathrm{P})$ & 110.3 \\
\hline $\mathrm{O}(009)-\mathrm{C}(02 \mathrm{U})-\mathrm{C}(036)$ & $107.0(4)$ \\
\hline $\mathrm{H}(02 \mathrm{~N})-\mathrm{C}(02 \mathrm{U})-\mathrm{H}(02 \mathrm{P})$ & 108.6 \\
\hline $\mathrm{C}(036)-\mathrm{C}(02 \mathrm{U})-\mathrm{H}(02 \mathrm{~N})$ & 110.3 \\
\hline $\mathrm{C}(036)-\mathrm{C}(02 \mathrm{U})-\mathrm{H}(02 \mathrm{P})$ & 110.3 \\
\hline $\mathrm{O}(00 \mathrm{Q})-\mathrm{C}(02 \mathrm{~V})-\mathrm{C}(033)$ & $110.0(3)$ \\
\hline $\mathrm{O}(00 \mathrm{Q})-\mathrm{C}(02 \mathrm{~V})-\mathrm{C}(034)$ & $109.4(3)$ \\
\hline $\mathrm{O}(00 \mathrm{Q})-\mathrm{C}(02 \mathrm{~V})-\mathrm{C}(035)$ & $102.1(4)$ \\
\hline $\mathrm{C}(034)-\mathrm{C}(02 \mathrm{~V})-\mathrm{C}(033)$ & $112.5(5)$ \\
\hline $\mathrm{C}(035)-\mathrm{C}(02 \mathrm{~V})-\mathrm{C}(033)$ & $112.1(4)$ \\
\hline $\mathrm{C}(035)-\mathrm{C}(02 \mathrm{~V})-\mathrm{C}(034)$ & $110.4(5)$ \\
\hline $\mathrm{O}(00 \mathrm{R})-\mathrm{C}(02 \mathrm{~W})-\mathrm{C}(02 \mathrm{X})$ & $109.5(3$ \\
\hline $\mathrm{O}(00 \mathrm{R})-\mathrm{C}(02 \mathrm{~W})-\mathrm{C}(02 \mathrm{Z})$ & $101.6(3)$ \\
\hline $\mathrm{O}(00 \mathrm{R})-\mathrm{C}(02 \mathrm{~W})-\mathrm{C}(032)$ & $110.6(4)$ \\
\hline $\mathrm{C}(02 \mathrm{Z})-\mathrm{C}(02 \mathrm{~W})-\mathrm{C}(02 \mathrm{X})$ & $109.6(5)$ \\
\hline $\mathrm{C}(032)-\mathrm{C}(02 \mathrm{~W})-\mathrm{C}(02 \mathrm{X})$ & $112.6(4)$ \\
\hline $\mathrm{C}(032)-\mathrm{C}(02 \mathrm{~W})-\mathrm{C}(02 \mathrm{Z})$ & $112.4(5)$ \\
\hline $\mathrm{C}(02 \mathrm{~W})-\mathrm{C}(02 \mathrm{X})-\mathrm{H}(3 \mathrm{AA})$ & 109.5 \\
\hline $\mathrm{C}(02 \mathrm{~W})-\mathrm{C}(02 \mathrm{X})-\mathrm{HF}$ & 109.5 \\
\hline $\mathrm{C}(02 \mathrm{~W})-\mathrm{C}(02 \mathrm{X})-\mathrm{HG}$ & 109.5 \\
\hline $\mathrm{H}(3 \mathrm{AA})-\mathrm{C}(02 \mathrm{X})-\mathrm{HF}$ & 109.5 \\
\hline $\mathrm{H}(3 \mathrm{AA})-\mathrm{C}(02 \mathrm{X})-\mathrm{HG}$ & 109.5 \\
\hline $\mathrm{HF}-\mathrm{C}(02 \mathrm{X})-\mathrm{HG}$ & 109.5 \\
\hline $\mathrm{O}(00 \mathrm{~N})-\mathrm{C}(02 \mathrm{Y})-\mathrm{H}(4 \mathrm{AA})$ & 124.2 \\
\hline $\mathrm{C}(2)-\mathrm{C}(02 \mathrm{Y})-\mathrm{O}(00 \mathrm{~N})$ & $111.6(5)$ \\
\hline $\mathrm{C}(2)-\mathrm{C}(02 \mathrm{Y})-\mathrm{H}(4 \mathrm{AA})$ & 124.2 \\
\hline
\end{tabular}




\begin{tabular}{|c|c|}
\hline $\mathrm{C}(02 \mathrm{~W})-\mathrm{C}(02 \mathrm{Z})-\mathrm{H}(5 \mathrm{AA})$ & 109.5 \\
\hline $\mathrm{C}(02 \mathrm{~W})-\mathrm{C}(02 \mathrm{Z})-\mathrm{HH}$ & 109.5 \\
\hline $\mathrm{C}(02 \mathrm{~W})-\mathrm{C}(02 \mathrm{Z})-\mathrm{HI}$ & 109.5 \\
\hline $\mathrm{H}(5 \mathrm{AA})-\mathrm{C}(02 \mathrm{Z})-\mathrm{HH}$ & 109.5 \\
\hline $\mathrm{H}(5 \mathrm{AA})-\mathrm{C}(02 \mathrm{Z})-\mathrm{HI}$ & 109.5 \\
\hline HH-C(02Z)-HI & 109.5 \\
\hline $\mathrm{C}(02 \mathrm{Q})-\mathrm{C}(030)-\mathrm{H}(03 \mathrm{R})$ & 109.5 \\
\hline $\mathrm{C}(02 \mathrm{Q})-\mathrm{C}(030)-\mathrm{H}(03 \mathrm{~S})$ & 109.5 \\
\hline $\mathrm{C}(02 \mathrm{Q})-\mathrm{C}(030)-\mathrm{H}(03 \mathrm{~T})$ & 109.5 \\
\hline $\mathrm{H}(03 \mathrm{R})-\mathrm{C}(030)-\mathrm{H}(03 \mathrm{~S})$ & 109.5 \\
\hline $\mathrm{H}(03 \mathrm{R})-\mathrm{C}(030)-\mathrm{H}(03 \mathrm{~T})$ & 109.5 \\
\hline $\mathrm{H}(03 \mathrm{~S})-\mathrm{C}(030)-\mathrm{H}(03 \mathrm{~T})$ & 109.5 \\
\hline $\mathrm{O}(00 \mathrm{M})-\mathrm{C}(031)-\mathrm{H}(03 \mathrm{D})$ & 110.2 \\
\hline $\mathrm{O}(00 \mathrm{M})-\mathrm{C}(031)-\mathrm{H}(03 \mathrm{E})$ & 110.2 \\
\hline $\mathrm{H}(03 \mathrm{D})-\mathrm{C}(031)-\mathrm{H}(03 \mathrm{E})$ & 108.5 \\
\hline $\mathrm{C}(037)-\mathrm{C}(031)-\mathrm{O}(00 \mathrm{M})$ & $107.7(5)$ \\
\hline $\mathrm{C}(037)-\mathrm{C}(031)-\mathrm{H}(03 \mathrm{D})$ & 110.2 \\
\hline $\mathrm{C}(037)-\mathrm{C}(031)-\mathrm{H}(03 \mathrm{E})$ & 110.2 \\
\hline $\mathrm{C}(02 \mathrm{~W})-\mathrm{C}(032)-\mathrm{H}(03 \mathrm{U})$ & 109.5 \\
\hline $\mathrm{C}(02 \mathrm{~W})-\mathrm{C}(032)-\mathrm{H}(03 \mathrm{~V})$ & 109.5 \\
\hline $\mathrm{C}(02 \mathrm{~W})-\mathrm{C}(032)-\mathrm{H}(03 \mathrm{~W})$ & 109.5 \\
\hline $\mathrm{H}(03 \mathrm{U})-\mathrm{C}(032)-\mathrm{H}(03 \mathrm{~V})$ & 109.5 \\
\hline $\mathrm{H}(03 \mathrm{U})-\mathrm{C}(032)-\mathrm{H}(03 \mathrm{~W})$ & 109.5 \\
\hline $\mathrm{H}(03 \mathrm{~V})-\mathrm{C}(032)-\mathrm{H}(03 \mathrm{~W})$ & 109.5 \\
\hline $\mathrm{C}(02 \mathrm{~V})-\mathrm{C}(033)-\mathrm{H}(03 \mathrm{~F})$ & 109.5 \\
\hline $\mathrm{C}(02 \mathrm{~V})-\mathrm{C}(033)-\mathrm{H}(03 \mathrm{G})$ & 109.5 \\
\hline $\mathrm{C}(02 \mathrm{~V})-\mathrm{C}(033)-\mathrm{H}(03 \mathrm{H})$ & 109.5 \\
\hline $\mathrm{H}(03 \mathrm{~F})-\mathrm{C}(033)-\mathrm{H}(03 \mathrm{G})$ & 109.5 \\
\hline $\mathrm{H}(03 \mathrm{~F})-\mathrm{C}(033)-\mathrm{H}(03 \mathrm{H})$ & 109.5 \\
\hline $\mathrm{H}(03 \mathrm{G})-\mathrm{C}(033)-\mathrm{H}(03 \mathrm{H})$ & 109.5 \\
\hline $\mathrm{C}(02 \mathrm{~V})-\mathrm{C}(034)-\mathrm{H}(03 \mathrm{I})$ & 109.5 \\
\hline $\mathrm{C}(02 \mathrm{~V})-\mathrm{C}(034)-\mathrm{H}(03 \mathrm{~J})$ & 109.5 \\
\hline $\mathrm{C}(02 \mathrm{~V})-\mathrm{C}(034)-\mathrm{H}(03 \mathrm{~K})$ & 109.5 \\
\hline $\mathrm{H}(03 \mathrm{I})-\mathrm{C}(034)-\mathrm{H}(03 \mathrm{~J})$ & 109.5 \\
\hline $\mathrm{H}(03 \mathrm{I})-\mathrm{C}(034)-\mathrm{H}(03 \mathrm{~K})$ & 109.5 \\
\hline $\mathrm{H}(03 \mathrm{~J})-\mathrm{C}(034)-\mathrm{H}(03 \mathrm{~K})$ & 109.5 \\
\hline $\mathrm{C}(02 \mathrm{~V})-\mathrm{C}(035)-\mathrm{H}(03 \mathrm{~L})$ & 109.5 \\
\hline $\mathrm{C}(02 \mathrm{~V})-\mathrm{C}(035)-\mathrm{H}(03 \mathrm{M})$ & 109.5 \\
\hline
\end{tabular}




\begin{tabular}{lc} 
C(02V)-C(035)-H(03N) & 109.5 \\
H(03L)-C(035)-H(03M) & 109.5 \\
H(03L)-C(035)-H(03N) & 109.5 \\
H(03M)-C(035)-H(03N) & 109.5 \\
C(02U)-C(036)-H(03A) & 109.5 \\
C(02U)-C(036)-H(03B) & 109.5 \\
C(02U)-C(036)-H(03C) & 109.5 \\
H(03A)-C(036)-H(03B) & 109.5 \\
H(03A)-C(036)-H(03C) & 109.5 \\
H(03B)-C(036)-H(03C) & 109.5 \\
C(031)-C(037)-H(03O) & 109.5 \\
C(031)-C(037)-H(03P) & 109.5 \\
C(031)-C(037)-H(03Q) & 109.5 \\
H(03O)-C(037)-H(03P) & 109.5 \\
H(03O)-C(037)-H(03Q) & 109.5 \\
H(03P)-C(037)-H(03Q) & 109.5 \\
C(02Y)-C(2)-H(2A) & 109.5 \\
C(02Y)-C(2)-H(2B) & 109.5 \\
C(02Y)-C(2)-H(2C) & 109.5 \\
H(2A)-C(2)-H(2B) & 109.5 \\
H(2A)-C(2)-H(2C) & 109.5 \\
H(2B)-C(2)-H(2C) & 109.5 \\
\hline
\end{tabular}

Symmetry transformations used to generate equivalent atoms:

Table 4. Anisotropic displacement parameters $\left(A^{\wedge} 2 \times 10^{\wedge} 3\right)$ for $3 a$.

The anisotropic displacement factor exponent takes the form:

$-2 \mathrm{pi}^{\wedge} 2\left[h^{\wedge} 2 a^{* \wedge} \mathrm{U} U 11+\ldots+2 \mathrm{~h} \mathrm{k} a^{*} b^{*} \mathrm{U12}\right]$

\begin{tabular}{|c|c|c|c|c|c|c|}
\hline \multicolumn{2}{|c|}{ U11 } & \multirow{2}{*}{$\begin{array}{l}\mathrm{U} 22 \\
52(1)\end{array}$} & \multirow{2}{*}{$\begin{array}{l}\text { U33 } \\
50(1)\end{array}$} & \multirow{2}{*}{$\begin{array}{l}\mathrm{U} 23 \\
13(1)\end{array}$} & \multirow{2}{*}{$\begin{array}{l}\text { U13 } \\
17(1)\end{array}$} & \multirow{2}{*}{$\begin{array}{l}\mathrm{U} 12 \\
0(1)\end{array}$} \\
\hline $\mathrm{F}(001)$ & 81(1) & & & & & \\
\hline $\mathrm{F}(002)$ & 71(1) & $54(1)$ & $63(1)$ & $0(1)$ & $21(1)$ & $18(1)$ \\
\hline $\mathrm{F}(003)$ & $50(1)$ & $59(1)$ & $65(1)$ & $5(1)$ & $-3(1)$ & $14(1)$ \\
\hline $\mathrm{F}(004)$ & $56(1)$ & $64(1)$ & $80(1)$ & $3(1)$ & $24(1)$ & $-7(1)$ \\
\hline $\mathrm{F}(005)$ & $73(1)$ & $53(1)$ & $66(1)$ & $-16(1)$ & $14(1)$ & $8(1)$ \\
\hline $\mathrm{O}(006)$ & $41(1)$ & $34(1)$ & $49(1)$ & $-5(1)$ & $10(1)$ & $-1(1)$ \\
\hline $\mathrm{O}(007)$ & $47(1)$ & $53(1)$ & $37(1)$ & $14(1)$ & $0(1)$ & $-3(1)$ \\
\hline $\mathrm{F}(008)$ & $83(1)$ & $68(1)$ & $47(1)$ & $-4(1)$ & $-14(1)$ & $7(1)$ \\
\hline
\end{tabular}




\begin{tabular}{|c|c|c|c|c|c|c|}
\hline $\mathrm{O}(009)$ & $62(1)$ & $39(1)$ & $52(1)$ & $-10(1)$ & $6(1)$ & $-4(1)$ \\
\hline $\mathrm{O}(00 \mathrm{~A})$ & $38(1)$ & $35(1)$ & $43(1)$ & $-2(1)$ & $2(1)$ & $-1(1)$ \\
\hline $\mathrm{F}(00 \mathrm{~B})$ & $82(1)$ & $52(1)$ & $56(1)$ & $15(1)$ & $-10(1)$ & $0(1)$ \\
\hline $\mathrm{F}(00 \mathrm{C})$ & $78(1)$ & $55(1)$ & $73(1)$ & 13(1) & $5(1)$ & $24(1)$ \\
\hline $\mathrm{O}(00 \mathrm{D})$ & $51(1)$ & $32(1)$ & $32(1)$ & $-1(1)$ & $2(1)$ & $-3(1)$ \\
\hline $\mathrm{F}(00 \mathrm{E})$ & $101(2)$ & $64(1)$ & $44(1)$ & $-6(1)$ & $22(1)$ & $-14(1)$ \\
\hline $\mathrm{O}(00 \mathrm{~F})$ & $57(1)$ & $39(1)$ & $47(1)$ & $8(1)$ & $-1(1)$ & $-10(1)$ \\
\hline $\mathrm{O}(00 \mathrm{G})$ & $56(1)$ & $36(1)$ & $30(1)$ & $1(1)$ & $-5(1)$ & $-5(1)$ \\
\hline $\mathrm{F}(00 \mathrm{H})$ & $82(1)$ & $65(1)$ & 71(1) & $23(1)$ & $-1(1)$ & $26(1)$ \\
\hline $\mathrm{F}(00 \mathrm{I})$ & $46(1)$ & 73(2) & 101(2) & $8(1)$ & $-5(1)$ & $-6(1)$ \\
\hline $\mathrm{O}(00 \mathrm{~J})$ & $42(1)$ & $65(2)$ & $43(1)$ & $-9(1)$ & $6(1)$ & $-19(1)$ \\
\hline $\mathrm{O}(00 \mathrm{~K})$ & $49(1)$ & $58(2)$ & $64(1)$ & $-3(1)$ & $-8(1)$ & $-10(1)$ \\
\hline $\mathrm{O}(00 \mathrm{~L})$ & $57(1)$ & 71(2) & $42(1)$ & $12(1)$ & $0(1)$ & $16(1)$ \\
\hline $\mathrm{O}(00 \mathrm{M})$ & $72(2)$ & $37(1)$ & $62(1)$ & $4(1)$ & $3(1)$ & $-13(1)$ \\
\hline $\mathrm{O}(00 \mathrm{~N})$ & $65(1)$ & $42(1)$ & $58(1)$ & $-13(1)$ & $3(1)$ & $-1(1)$ \\
\hline $\mathrm{O}(00 \mathrm{O})$ & $52(1)$ & $70(2)$ & $56(1)$ & $24(1)$ & $2(1)$ & $-14(1)$ \\
\hline $\mathrm{F}(00 \mathrm{P})$ & $89(2)$ & $55(1)$ & $74(1)$ & 13(1) & 15(1) & $-25(1)$ \\
\hline $\mathrm{O}(00 \mathrm{Q})$ & $74(2)$ & $61(2)$ & $46(1)$ & $-19(1)$ & $-1(1)$ & 1(1) \\
\hline $\mathrm{O}(00 \mathrm{R})$ & $62(1)$ & $66(2)$ & $39(1)$ & $3(1)$ & $2(1)$ & $30(1)$ \\
\hline $\mathrm{O}(00 \mathrm{~S})$ & $80(2)$ & $64(2)$ & $51(1)$ & $-10(1)$ & $23(1)$ & $4(1)$ \\
\hline $\mathrm{O}(00 \mathrm{~T})$ & $68(2)$ & $86(2)$ & $47(1)$ & $-20(1)$ & $6(1)$ & $-32(1)$ \\
\hline $\mathrm{N}(00 \mathrm{U})$ & $36(1)$ & $50(1)$ & $33(1)$ & $9(1)$ & $1(1)$ & $-5(1)$ \\
\hline $\mathrm{N}(00 \mathrm{~V})$ & $39(1)$ & $32(1)$ & $57(1)$ & $-1(1)$ & $7(1)$ & $-2(1)$ \\
\hline $\mathrm{N}(00 \mathrm{~W})$ & $43(1)$ & $46(1)$ & $42(1)$ & $-6(1)$ & $-1(1)$ & $-8(1)$ \\
\hline $\mathrm{N}(00 \mathrm{X})$ & $43(1)$ & $47(1)$ & $34(1)$ & $1(1)$ & $4(1)$ & $7(1)$ \\
\hline $\mathrm{N}(00 \mathrm{Y})$ & $39(1)$ & $56(2)$ & $38(1)$ & $-5(1)$ & $2(1)$ & $-10(1)$ \\
\hline $\mathrm{O}(00 \mathrm{Z})$ & $89(2)$ & $72(2)$ & $72(2)$ & $16(1)$ & $35(2)$ & $-15(2)$ \\
\hline $\mathrm{N}(010)$ & $37(1)$ & $36(1)$ & $59(1)$ & $0(1)$ & $5(1)$ & $0(1)$ \\
\hline $\mathrm{C}(011)$ & $42(1)$ & $28(1)$ & $32(1)$ & $-2(1)$ & 1(1) & $-1(1)$ \\
\hline $\mathrm{O}(012)$ & $74(2)$ & $87(2)$ & $69(2)$ & $-24(2)$ & $-20(1)$ & $-7(2)$ \\
\hline $\mathrm{O}(013)$ & $65(2)$ & $81(2)$ & $101(2)$ & $-36(2)$ & $-10(2)$ & $-19(2)$ \\
\hline $\mathrm{C}(014)$ & $40(1)$ & $33(1)$ & $31(1)$ & $-1(1)$ & $7(1)$ & $-1(1)$ \\
\hline $\mathrm{N}(015)$ & $83(2)$ & $34(1)$ & $31(1)$ & $1(1)$ & $-4(1)$ & $0(1)$ \\
\hline $\mathrm{C}(016)$ & $44(1)$ & $39(2)$ & $38(1)$ & $-4(1)$ & 2(1) & $-2(1)$ \\
\hline $\mathrm{C}(017)$ & $39(1)$ & $31(1)$ & $34(1)$ & $-3(1)$ & $6(1)$ & $-2(1)$ \\
\hline $\mathrm{C}(018)$ & $38(1)$ & $35(1)$ & $31(1)$ & $1(1)$ & $1(1)$ & $-1(1)$ \\
\hline $\mathrm{C}(019)$ & $38(1)$ & $36(1)$ & $32(1)$ & $-1(1)$ & $-2(1)$ & $-4(1)$ \\
\hline $\mathrm{C}(01 \mathrm{~A})$ & $38(1)$ & $42(2)$ & $34(1)$ & $0(1)$ & $-1(1)$ & $-5(1)$ \\
\hline
\end{tabular}




\begin{tabular}{|c|c|c|c|c|c|c|}
\hline $\mathrm{C}(01 \mathrm{~B})$ & $38(1)$ & $36(2)$ & $32(1)$ & $0(1)$ & $3(1)$ & $0(1)$ \\
\hline $\mathrm{N}(01 \mathrm{C})$ & $64(2)$ & $35(1)$ & $28(1)$ & $2(1)$ & $-3(1)$ & $-2(1)$ \\
\hline $\mathrm{C}(01 \mathrm{D})$ & $45(2)$ & $54(2)$ & $30(1)$ & $6(1)$ & $3(1)$ & $-6(1)$ \\
\hline $\mathrm{C}(01 \mathrm{E})$ & $40(1)$ & $31(1)$ & $33(1)$ & $-4(1)$ & $8(1)$ & $-2(1)$ \\
\hline $\mathrm{C}(01 \mathrm{~F})$ & $42(1)$ & $35(1)$ & $32(1)$ & $-1(1)$ & $0(1)$ & $-1(1)$ \\
\hline $\mathrm{C}(01 \mathrm{G})$ & $56(2)$ & $31(1)$ & $43(2)$ & $-3(1)$ & $8(1)$ & $4(1)$ \\
\hline $\mathrm{C}(01 \mathrm{H})$ & $44(1)$ & $30(1)$ & $36(1)$ & $-2(1)$ & $4(1)$ & $0(1)$ \\
\hline $\mathrm{C}(01 \mathrm{I})$ & $44(1)$ & $36(2)$ & $32(1)$ & $-2(1)$ & $4(1)$ & $-1(1)$ \\
\hline $\mathrm{C}(01 \mathrm{~J})$ & $55(2)$ & $40(2)$ & $53(2)$ & 11(1) & 11(1) & $2(1)$ \\
\hline $\mathrm{C}(01 \mathrm{~K})$ & $44(2)$ & $58(2)$ & $43(2)$ & $-8(1)$ & $4(1)$ & $-9(1)$ \\
\hline $\mathrm{C}(01 \mathrm{~L})$ & $51(2)$ & $37(2)$ & $37(1)$ & $5(1)$ & $6(1)$ & $2(1)$ \\
\hline $\mathrm{C}(01 \mathrm{M})$ & $38(1)$ & $35(1)$ & $29(1)$ & $1(1)$ & $-3(1)$ & $-3(1)$ \\
\hline $\mathrm{C}(01 \mathrm{~N})$ & $51(2)$ & $44(2)$ & $47(2)$ & $-1(1)$ & $8(1)$ & $2(1)$ \\
\hline $\mathrm{C}(01 \mathrm{O})$ & $42(1)$ & $33(1)$ & $40(1)$ & $-2(1)$ & $6(1)$ & 1(1) \\
\hline $\mathrm{C}(01 \mathrm{P})$ & $59(2)$ & $50(2)$ & $57(2)$ & $-11(2)$ & $-6(2)$ & $-3(2)$ \\
\hline $\mathrm{C}(01 \mathrm{Q})$ & $54(2)$ & $45(2)$ & $40(2)$ & $4(1)$ & $10(1)$ & $12(1)$ \\
\hline $\mathrm{C}(01 \mathrm{R})$ & $42(1)$ & $35(2)$ & $42(1)$ & $0(1)$ & $0(1)$ & $-3(1)$ \\
\hline $\mathrm{C}(01 \mathrm{~S})$ & $58(2)$ & $42(2)$ & $39(1)$ & $-1(1)$ & $8(1)$ & 1(1) \\
\hline $\mathrm{C}(01 \mathrm{~T})$ & $51(2)$ & $47(2)$ & $53(2)$ & $4(1)$ & 1(1) & $7(1)$ \\
\hline $\mathrm{C}(01 \mathrm{U})$ & $39(1)$ & $33(1)$ & $46(2)$ & $5(1)$ & $9(1)$ & $-2(1)$ \\
\hline $\mathrm{C}(01 \mathrm{~V})$ & $45(1)$ & $36(1)$ & $35(1)$ & $-4(1)$ & $4(1)$ & $3(1)$ \\
\hline $\mathrm{C}(01 \mathrm{~W})$ & $48(2)$ & $45(2)$ & $85(2)$ & $6(2)$ & $25(2)$ & $4(1)$ \\
\hline $\mathrm{C}(01 \mathrm{X})$ & $59(2)$ & $44(2)$ & $47(2)$ & $1(1)$ & $6(1)$ & $9(1)$ \\
\hline $\mathrm{C}(01 \mathrm{Y})$ & $42(1)$ & $39(2)$ & $30(1)$ & $2(1)$ & $-1(1)$ & $-3(1)$ \\
\hline $\mathrm{C}(01 \mathrm{Z})$ & $41(2)$ & $60(2)$ & $54(2)$ & $-10(2)$ & $10(1)$ & $-2(1)$ \\
\hline $\mathrm{C}(020)$ & $44(1)$ & $44(2)$ & $39(1)$ & $-6(1)$ & $4(1)$ & $-1(1)$ \\
\hline $\mathrm{C}(021)$ & $51(2)$ & $40(2)$ & $49(2)$ & $-4(1)$ & $-1(1)$ & $-9(1)$ \\
\hline $\mathrm{C}(022)$ & $45(1)$ & $47(2)$ & $39(1)$ & $-2(1)$ & $-6(1)$ & $0(1)$ \\
\hline $\mathrm{C}(023)$ & $52(2)$ & $42(2)$ & $38(1)$ & $1(1)$ & $-2(1)$ & $6(1)$ \\
\hline $\mathrm{C}(024)$ & $40(1)$ & $35(1)$ & $49(2)$ & $4(1)$ & $4(1)$ & $-6(1)$ \\
\hline $\mathrm{C}(025)$ & $54(2)$ & $39(2)$ & $55(2)$ & $2(1)$ & $18(1)$ & $2(1)$ \\
\hline $\mathrm{C}(026)$ & $37(1)$ & $49(2)$ & $47(2)$ & $1(1)$ & $2(1)$ & $-10(1)$ \\
\hline $\mathrm{C}(027)$ & $45(2)$ & $53(2)$ & $67(2)$ & $1(2)$ & $-8(1)$ & $-3(1)$ \\
\hline $\mathrm{C}(028)$ & $41(2)$ & $57(2)$ & $58(2)$ & $-7(2)$ & $-6(1)$ & $5(1)$ \\
\hline $\mathrm{C}(029)$ & $75(2)$ & $47(2)$ & $36(2)$ & $7(1)$ & $16(1)$ & $15(2)$ \\
\hline $\mathrm{C}(02 \mathrm{~A})$ & $48(2)$ & $46(2)$ & $37(1)$ & $6(1)$ & $3(1)$ & $6(1)$ \\
\hline $\mathrm{C}(02 \mathrm{~B})$ & $64(2)$ & $44(2)$ & $47(2)$ & $5(1)$ & $9(1)$ & $-9(1)$ \\
\hline $\mathrm{C}(02 \mathrm{C})$ & $45(2)$ & $55(2)$ & $38(1)$ & $-2(1)$ & $6(1)$ & $-7(1)$ \\
\hline
\end{tabular}




\begin{tabular}{|c|c|c|c|c|c|c|}
\hline$C(02 D)$ & $57(2)$ & 71(2) & $45(2)$ & 2(2) & $6(1)$ & $-14(2)$ \\
\hline $\mathrm{C}(02 \mathrm{E})$ & $52(2)$ & 71(2) & $53(2)$ & $6(2)$ & $-6(1)$ & $-2(2)$ \\
\hline $\mathrm{C}(02 \mathrm{~F})$ & $37(2)$ & $53(2)$ & $100(3)$ & $4(2)$ & $8(2)$ & 2(1) \\
\hline $\mathrm{C}(02 \mathrm{G})$ & $85(2)$ & $40(2)$ & $35(1)$ & $4(1)$ & $0(1)$ & $4(2)$ \\
\hline $\mathrm{C}(02 \mathrm{H})$ & 71(2) & $49(2)$ & $32(1)$ & $1(1)$ & $-6(1)$ & $8(2)$ \\
\hline $\mathrm{C}(02 \mathrm{I})$ & $62(2)$ & $55(2)$ & $34(1)$ & $12(1)$ & 1(1) & $12(2)$ \\
\hline $\mathrm{C}(02 \mathrm{~J})$ & $49(2)$ & $65(2)$ & $60(2)$ & $10(2)$ & $3(2)$ & $-2(2)$ \\
\hline $\mathrm{C}(02 \mathrm{~K})$ & $42(2)$ & $37(2)$ & $64(2)$ & $-2(1)$ & $13(1)$ & $3(1)$ \\
\hline $\mathrm{C}(02 \mathrm{~L})$ & $64(2)$ & $38(2)$ & $31(1)$ & $-2(1)$ & $-5(1)$ & $-3(1)$ \\
\hline $\mathrm{C}(02 \mathrm{M})$ & $71(2)$ & $75(3)$ & $46(2)$ & $3(2)$ & $-17(2)$ & $-2(2)$ \\
\hline $\mathrm{C}(02 \mathrm{~N})$ & $40(2)$ & $40(2)$ & $77(2)$ & $-2(2)$ & $6(1)$ & $-6(1)$ \\
\hline $\mathrm{C}(02 \mathrm{O})$ & $80(2)$ & $53(2)$ & $38(2)$ & $6(1)$ & $0(2)$ & $6(2)$ \\
\hline $\mathrm{C}(02 \mathrm{P})$ & $64(2)$ & $76(3)$ & $54(2)$ & $6(2)$ & $-12(2)$ & $12(2)$ \\
\hline $\mathrm{C}(02 \mathrm{Q})$ & $78(2)$ & $47(2)$ & $62(2)$ & $7(2)$ & $12(2)$ & $-23(2)$ \\
\hline $\mathrm{C}(02 \mathrm{R})$ & $89(3)$ & $87(3)$ & $38(2)$ & $6(2)$ & $6(2)$ & $31(2)$ \\
\hline $\mathrm{C}(02 \mathrm{~S})$ & $69(2)$ & $48(2)$ & $68(2)$ & $-3(2)$ & $-6(2)$ & $-9(2)$ \\
\hline $\mathrm{C}(02 \mathrm{~T})$ & $73(2)$ & $58(2)$ & $59(2)$ & $19(2)$ & $18(2)$ & $8(2)$ \\
\hline $\mathrm{C}(02 \mathrm{U})$ & $80(2)$ & $49(2)$ & $77(2)$ & $-26(2)$ & $-8(2)$ & $0(2)$ \\
\hline $\mathrm{C}(02 \mathrm{~V})$ & $122(4)$ & $58(2)$ & $41(2)$ & $-17(2)$ & $-15(2)$ & $20(2)$ \\
\hline $\mathrm{C}(02 \mathrm{~W})$ & $69(2)$ & $67(2)$ & $54(2)$ & $4(2)$ & $6(2)$ & $36(2)$ \\
\hline $\mathrm{C}(02 \mathrm{X})$ & $60(2)$ & $84(3)$ & $96(3)$ & $1(2)$ & $14(2)$ & $19(2)$ \\
\hline $\mathrm{C}(02 \mathrm{Y})$ & $78(3)$ & 73(3) & $106(3)$ & $-52(3)$ & $17(2)$ & $-4(2)$ \\
\hline $\mathrm{C}(02 \mathrm{Z})$ & $132(5)$ & $152(6)$ & $65(3)$ & $1(3)$ & 23(3) & $94(5)$ \\
\hline $\mathrm{C}(030)$ & $130(4)$ & $54(2)$ & $75(3)$ & $25(2)$ & $-6(3)$ & $-20(3)$ \\
\hline $\mathrm{C}(031)$ & 99(3) & $59(3)$ & $99(3)$ & 21(2) & $-23(3)$ & $-33(2)$ \\
\hline $\mathrm{C}(032)$ & 91(3) & $62(3)$ & 112(4) & $4(2)$ & 1(3) & $30(2)$ \\
\hline $\mathrm{C}(033)$ & $151(5)$ & $52(2)$ & $78(3)$ & $-14(2)$ & $-30(3)$ & $20(3)$ \\
\hline $\mathrm{C}(034)$ & $165(6)$ & $82(3)$ & $64(3)$ & $-11(2)$ & $-42(3)$ & $31(4)$ \\
\hline $\mathrm{C}(035)$ & $149(5)$ & 91(4) & $49(2)$ & $-19(2)$ & $17(3)$ & $24(3)$ \\
\hline $\mathrm{C}(036)$ & $86(3)$ & $72(3)$ & $155(5)$ & $-45(3)$ & $8(3)$ & $-23(3)$ \\
\hline $\mathrm{C}(037)$ & $315(13)$ & $101(5)$ & $110(5)$ & $-23(4)$ & $-3(6)$ & $-125(7)$ \\
\hline$C(2)$ & $117(5)$ & $178(8)$ & $286(11)$ & $-169(8)$ & $6(6)$ & $17(5)$ \\
\hline
\end{tabular}


Table 5. Hydrogen coordinates $\left(x 1^{\wedge} 4\right)$ and isotropic displacement parameters $\left(A^{\wedge} 2 x\right.$ $\left.10^{\wedge} 3\right)$ for $3 a$.

\begin{tabular}{|c|c|c|c|c|}
\hline & $\mathrm{x}$ & $\mathrm{y}$ & $\mathrm{z}$ & $\mathrm{U}(\mathrm{eq})$ \\
\hline $\mathrm{H}(00 \mathrm{U})$ & 3526 & 3809 & 7618 & 47 \\
\hline $\mathrm{H}(00 \mathrm{~V})$ & 4920 & 7998 & 2630 & 51 \\
\hline $\mathrm{H}(00 \mathrm{~W})$ & 7717 & 3586 & 7204 & 53 \\
\hline $\mathrm{H}(00 \mathrm{X})$ & 3064 & 6247 & 7151 & 49 \\
\hline $\mathrm{H}(00 \mathrm{Y})$ & 2122 & 6257 & 3251 & 53 \\
\hline $\mathrm{H}(010)$ & 6005 & 4677 & 7328 & 52 \\
\hline $\mathrm{H}(014)$ & 7240 & 3418 & 6184 & 42 \\
\hline $\mathrm{H}(015)$ & 6993 & 2098 & 4388 & 59 \\
\hline $\mathrm{H}(018)$ & 4144 & 5980 & 7036 & 41 \\
\hline $\mathrm{H}(01 \mathrm{C})$ & 2921 & 5411 & 4768 & 51 \\
\hline $\mathrm{H}(01 \mathrm{E})$ & 3214 & 6604 & 3180 & 42 \\
\hline $\mathrm{H}(01 \mathrm{M})$ & 3530 & 4128 & 6536 & 41 \\
\hline $\mathrm{H}(01 \mathrm{Q})$ & 2419 & 6103 & 8890 & 55 \\
\hline $\mathrm{H}(01 \mathrm{~S})$ & 3523 & 7307 & 1353 & 55 \\
\hline $\mathrm{H}(01 \mathrm{~W})$ & 10035 & 2633 & 5312 & 70 \\
\hline $\mathrm{H}(01 \mathrm{Z})$ & 698 & 4676 & 7905 & 62 \\
\hline $\mathrm{H}(022)$ & 1688 & 5040 & 5996 & 53 \\
\hline $\mathrm{H}(023)$ & 5014 & 5431 & 8774 & 53 \\
\hline $\mathrm{H}(025)$ & 8734 & 2772 & 5003 & 59 \\
\hline $\mathrm{H}(027)$ & 9666 & 3482 & 7356 & 66 \\
\hline $\mathrm{H}(028)$ & 638 & 5218 & 6703 & 63 \\
\hline $\mathrm{H}(029)$ & 3125 & 5645 & 9927 & 63 \\
\hline $\mathrm{H}(02 \mathrm{~T})$ & 1796 & 3943 & 8373 & 55 \\
\hline $\mathrm{H}(02 \mathrm{~A})$ & 938 & 4744 & 4943 & 86 \\
\hline $\mathrm{H}(02 \mathrm{~B})$ & 35 & 4816 & 4912 & 86 \\
\hline $\mathrm{H}(02 \mathrm{C})$ & 536 & 5704 & 4804 & 86 \\
\hline $\mathrm{H}(02 \mathrm{E})$ & 1046 & 6267 & 1647 & 71 \\
\hline $\mathrm{H}(02 \mathrm{Q})$ & 10493 & 2999 & 6479 & 76 \\
\hline $\mathrm{H}(02 \mathrm{R})$ & 6816 & 3686 & 4119 & 64 \\
\hline $\mathrm{H}(02 \mathrm{~S})$ & 7719 & 3531 & 4151 & 64 \\
\hline $\mathrm{H}(1 \mathrm{AA})$ & 4426 & 5328 & 9889 & 61 \\
\hline $\mathrm{H}(02 \mathrm{D})$ & -174 & 6011 & 3604 & 87 \\
\hline $\mathrm{H}(02 \mathrm{~F})$ & -679 & 5128 & 3722 & 87 \\
\hline
\end{tabular}




\begin{tabular}{|c|c|c|c|c|}
\hline $\mathrm{H}(02 \mathrm{G})$ & -228 & 5236 & 3002 & 87 \\
\hline $\mathrm{H}(02 \mathrm{H})$ & 5281 & 6436 & 2875 & 57 \\
\hline $\mathrm{H}(02 \mathrm{I})$ & 5042 & 6520 & 2030 & 57 \\
\hline $\mathrm{H}(02 \mathrm{U})$ & 2957 & 3799 & 4499 & 54 \\
\hline $\mathrm{H}(02 \mathrm{~V})$ & 2141 & 3982 & 4818 & 54 \\
\hline $\mathrm{H}(02 \mathrm{M})$ & 1389 & 6825 & 526 & 78 \\
\hline $\mathrm{H}(2 \mathrm{AA})$ & 6271 & 6250 & 7069 & 63 \\
\hline $\mathrm{H}$ & 6195 & 6178 & 7928 & 63 \\
\hline $\mathrm{H}(02 \mathrm{O})$ & 2624 & 7353 & 371 & 68 \\
\hline $\mathrm{H}(02 \mathrm{~W})$ & 5114 & 2326 & 9099 & 98 \\
\hline $\mathrm{H}(02 \mathrm{X})$ & 4966 & 2330 & 9942 & 98 \\
\hline $\mathrm{H}(02 \mathrm{Y})$ & 4893 & 3250 & 9487 & 98 \\
\hline $\mathrm{H}(02 \mathrm{Z})$ & 1759 & 1302 & 6269 & 74 \\
\hline HA & 1729 & 1862 & 7009 & 74 \\
\hline $\mathrm{H}(02)$ & 3499 & 3513 & 9803 & 107 \\
\hline $\mathrm{HB}$ & 3648 & 2680 & 10343 & 107 \\
\hline $\mathrm{HC}$ & 2941 & 2658 & 9772 & 107 \\
\hline $\mathrm{H}(02 \mathrm{~J})$ & 471 & 3748 & 3213 & 93 \\
\hline $\mathrm{H}(02 \mathrm{~K})$ & 55 & 3559 & 3943 & 93 \\
\hline $\mathrm{H}(02 \mathrm{~L})$ & 959 & 3588 & 3950 & 93 \\
\hline $\mathrm{H}(0 \mathrm{AA})$ & 3276 & 1261 & 9134 & 94 \\
\hline HD & 3969 & 1077 & 9702 & 94 \\
\hline $\mathrm{HE}$ & 4109 & 1111 & 8859 & 94 \\
\hline $\mathrm{H}(02 \mathrm{~N})$ & 3503 & 3863 & 1318 & 83 \\
\hline $\mathrm{H}(02 \mathrm{P})$ & 2826 & 4573 & 1160 & 83 \\
\hline $\mathrm{H}(3 \mathrm{AA})$ & 647 & 7191 & 7216 & 119 \\
\hline $\mathrm{HF}$ & 90 & 7540 & 7809 & 119 \\
\hline HG & 547 & 6613 & 7933 & 119 \\
\hline $\mathrm{H}(4 \mathrm{AA})$ & 4723 & 8711 & 8858 & 102 \\
\hline $\mathrm{H}(5 \mathrm{AA})$ & 1047 & 7195 & 9122 & 174 \\
\hline $\mathrm{HH}$ & 666 & 8173 & 9018 & 174 \\
\hline HI & 1559 & 8089 & 9180 & 174 \\
\hline $\mathrm{H}(03 \mathrm{R})$ & 2882 & 566 & 6709 & 130 \\
\hline $\mathrm{H}(03 \mathrm{~S})$ & 2227 & 395 & 7257 & 130 \\
\hline $\mathrm{H}(03 \mathrm{~T})$ & 2869 & 1136 & 7441 & 130 \\
\hline $\mathrm{H}(03 \mathrm{D})$ & 9125 & 5651 & 5862 & 104 \\
\hline $\mathrm{H}(03 \mathrm{E})$ & 8608 & 6363 & 5406 & 104 \\
\hline $\mathrm{H}(03 \mathrm{U})$ & 1773 & 9003 & 8045 & 132 \\
\hline
\end{tabular}




\begin{tabular}{lcccc}
$\mathrm{H}(03 \mathrm{~V})$ & 888 & 9057 & 7826 & 132 \\
$\mathrm{H}(03 \mathrm{~W})$ & 1460 & 8629 & 7278 & 132 \\
$\mathrm{H}(03 \mathrm{~F})$ & 8534 & 6107 & 8583 & 142 \\
$\mathrm{H}(03 \mathrm{G})$ & 7942 & 6398 & 9164 & 142 \\
$\mathrm{H}(03 \mathrm{H})$ & 7662 & 6242 & 8341 & 142 \\
$\mathrm{H}(03 \mathrm{I})$ & 8421 & 3933 & 9410 & 158 \\
$\mathrm{H}(03 \mathrm{~J})$ & 8469 & 4844 & 9878 & 158 \\
$\mathrm{H}(03 \mathrm{~K})$ & 8987 & 4727 & 9203 & 158 \\
$\mathrm{H}(03 \mathrm{~L})$ & 6688 & 5158 & 8896 & 144 \\
$\mathrm{H}(03 \mathrm{M})$ & 7079 & 5152 & 9691 & 144 \\
$\mathrm{H}(03 \mathrm{~N})$ & 6979 & 4225 & 9250 & 144 \\
$\mathrm{H}(03 \mathrm{~A})$ & 2794 & 3185 & 2234 & 157 \\
$\mathrm{H}(03 \mathrm{~B})$ & 2341 & 3125 & 1469 & 157 \\
$\mathrm{H}(03 \mathrm{C})$ & 2114 & 3872 & 2043 & 157 \\
$\mathrm{H}(03 \mathrm{O})$ & 8513 & 6054 & 6906 & 264 \\
$\mathrm{H}(03 \mathrm{P})$ & 8007 & 6770 & 6450 & 264 \\
$\mathrm{H}(03 \mathrm{Q})$ & 8899 & 6925 & 6566 & 264 \\
$\mathrm{H}(2 \mathrm{~A})$ & 3567 & 9214 & 7981 & 291 \\
$\mathrm{H}(2 \mathrm{~B})$ & 3543 & 9258 & 8840 & 291 \\
$\mathrm{H}(2 \mathrm{C})$ & 3277 & 8358 & 8419 & 291 \\
& & & & \\
\hline
\end{tabular}


Table 6. Torsion angles [deg] for $3 a$.

\begin{tabular}{|c|c|}
\hline $\mathrm{O}(006)-\mathrm{C}(017)-\mathrm{C}(01 \mathrm{E})-\mathrm{C}(01 \mathrm{O})$ & $-176.9(2)$ \\
\hline $\mathrm{O}(006)-\mathrm{C}(017)-\mathrm{C}(01 \mathrm{E})-\mathrm{C}(01 \mathrm{~V})$ & $58.7(3)$ \\
\hline $\mathrm{O}(009)-\mathrm{C}(01 \mathrm{G})-\mathrm{C}(01 \mathrm{O})-\mathrm{C}(01 \mathrm{E})$ & $-68.6(3)$ \\
\hline $\mathrm{O}(009)-\mathrm{C}(01 \mathrm{G})-\mathrm{C}(01 \mathrm{O})-\mathrm{C}(01 \mathrm{X})$ & $54.1(3)$ \\
\hline $\mathrm{O}(009)-\mathrm{C}(01 \mathrm{G})-\mathrm{C}(01 \mathrm{O})-\mathrm{C}(02 \mathrm{~K})$ & $174.4(2)$ \\
\hline $\mathrm{O}(00 \mathrm{~F})-\mathrm{C}(016)-\mathrm{C}(01 \mathrm{~F})-\mathrm{C}(01 \mathrm{M})$ & $-67.4(3)$ \\
\hline $\mathrm{O}(00 \mathrm{~F})-\mathrm{C}(016)-\mathrm{C}(01 \mathrm{~F})-\mathrm{C}(01 \mathrm{~N})$ & $54.5(3)$ \\
\hline $\mathrm{O}(00 \mathrm{~F})-\mathrm{C}(016)-\mathrm{C}(01 \mathrm{~F})-\mathrm{C}(02 \mathrm{~L})$ & $175.5(2)$ \\
\hline $\mathrm{O}(00 \mathrm{~K})-\mathrm{C}(016)-\mathrm{C}(01 \mathrm{~F})-\mathrm{C}(01 \mathrm{M})$ & $110.0(3)$ \\
\hline $\mathrm{O}(00 \mathrm{~K})-\mathrm{C}(016)-\mathrm{C}(01 \mathrm{~F})-\mathrm{C}(01 \mathrm{~N})$ & $-128.0(3)$ \\
\hline $\mathrm{O}(00 \mathrm{~K})-\mathrm{C}(016)-\mathrm{C}(01 \mathrm{~F})-\mathrm{C}(02 \mathrm{~L})$ & $-7.1(4)$ \\
\hline $\mathrm{O}(00 \mathrm{M})-\mathrm{C}(01 \mathrm{~J})-\mathrm{C}(01 \mathrm{~L})-\mathrm{C}(014)$ & $-61.9(3)$ \\
\hline $\mathrm{O}(00 \mathrm{M})-\mathrm{C}(01 \mathrm{~J})-\mathrm{C}(01 \mathrm{~L})-\mathrm{C}(01 \mathrm{~T})$ & $60.4(3)$ \\
\hline $\mathrm{O}(00 \mathrm{M})-\mathrm{C}(01 \mathrm{~J})-\mathrm{C}(01 \mathrm{~L})-\mathrm{C}(02 \mathrm{G})$ & $-178.8(3)$ \\
\hline $\mathrm{O}(00 \mathrm{~S})-\mathrm{C}(01 \mathrm{G})-\mathrm{C}(01 \mathrm{O})-\mathrm{C}(01 \mathrm{E})$ & 109.4(3) \\
\hline $\mathrm{O}(00 \mathrm{~S})-\mathrm{C}(01 \mathrm{G})-\mathrm{C}(01 \mathrm{O})-\mathrm{C}(01 \mathrm{X})$ & $-127.9(3)$ \\
\hline $\mathrm{O}(00 \mathrm{~S})-\mathrm{C}(01 \mathrm{G})-\mathrm{C}(01 \mathrm{O})-\mathrm{C}(02 \mathrm{~K})$ & $-7.6(4)$ \\
\hline $\mathrm{N}(00 \mathrm{U})-\mathrm{C}(01 \mathrm{~A})-\mathrm{C}(02 \mathrm{C})-\mathrm{C}(01 \mathrm{Z})$ & $176.5(3)$ \\
\hline $\mathrm{N}(00 \mathrm{~V})-\mathrm{C}(017)-\mathrm{C}(01 \mathrm{E})-\mathrm{C}(01 \mathrm{O})$ & $6.1(3)$ \\
\hline $\mathrm{N}(00 \mathrm{~V})-\mathrm{C}(017)-\mathrm{C}(01 \mathrm{E})-\mathrm{C}(01 \mathrm{~V})$ & $-118.3(2)$ \\
\hline $\mathrm{N}(00 \mathrm{~W})-\mathrm{C}(024)-\mathrm{C}(027)-\mathrm{C}(02 \mathrm{~F})$ & $-179.3(3)$ \\
\hline $\mathrm{N}(00 \mathrm{X})-\mathrm{C}(01 \mathrm{H})-\mathrm{C}(01 \mathrm{Q})-\mathrm{C}(029)$ & $-178.3(3)$ \\
\hline $\mathrm{N}(00 \mathrm{Y})-\mathrm{C}(020)-\mathrm{C}(02 \mathrm{E})-\mathrm{C}(02 \mathrm{M})$ & $179.7(4)$ \\
\hline $\mathrm{O}(00 \mathrm{Z})-\mathrm{C}(01 \mathrm{~J})-\mathrm{C}(01 \mathrm{~L})-\mathrm{C}(014)$ & $115.9(4)$ \\
\hline $\mathrm{O}(00 \mathrm{Z})-\mathrm{C}(01 \mathrm{~J})-\mathrm{C}(01 \mathrm{~L})-\mathrm{C}(01 \mathrm{~T})$ & $-121.8(4)$ \\
\hline $\mathrm{O}(00 \mathrm{Z})-\mathrm{C}(01 \mathrm{~J})-\mathrm{C}(01 \mathrm{~L})-\mathrm{C}(02 \mathrm{G})$ & $-1.0(5)$ \\
\hline $\mathrm{C}(011)-\mathrm{C}(018)-\mathrm{C}(01 \mathrm{~B})-\mathrm{O}(00 \mathrm{~A})$ & $61.2(3)$ \\
\hline $\mathrm{C}(011)-\mathrm{C}(018)-\mathrm{C}(01 \mathrm{~B})-\mathrm{N}(010)$ & $-117.6(2)$ \\
\hline $\mathrm{C}(011)-\mathrm{C}(018)-\mathrm{C}(01 \mathrm{R})-\mathrm{C}(021)$ & $-12.5(3)$ \\
\hline $\mathrm{C}(011)-\mathrm{C}(018)-\mathrm{C}(01 \mathrm{R})-\mathrm{C}(02 \mathrm{~B})$ & $-135.6(3)$ \\
\hline $\mathrm{C}(011)-\mathrm{C}(018)-\mathrm{C}(01 \mathrm{R})-\mathrm{C}(02 \mathrm{~N})$ & $107.5(3)$ \\
\hline $\mathrm{C}(011)-\mathrm{C}(01 \mathrm{H})-\mathrm{C}(01 \mathrm{Q})-\mathrm{C}(029)$ & $3.8(5)$ \\
\hline $\mathrm{C}(011)-\mathrm{C}(023)-\mathrm{C}(02 \mathrm{H})-\mathrm{C}(029)$ & $2.3(5)$ \\
\hline $\mathrm{C}(014)-\mathrm{C}(01 \mathrm{~L})-\mathrm{C}(01 \mathrm{~T})-\mathrm{F}(002)$ & $57.9(4)$ \\
\hline $\mathrm{C}(014)-\mathrm{C}(01 \mathrm{~L})-\mathrm{C}(01 \mathrm{~T})-\mathrm{F}(00 \mathrm{H})$ & $178.6(3)$ \\
\hline $\mathrm{C}(014)-\mathrm{C}(01 \mathrm{~L})-\mathrm{C}(01 \mathrm{~T})-\mathrm{F}(00 \mathrm{I})$ & $-62.4(3)$ \\
\hline
\end{tabular}




\begin{tabular}{|c|c|}
\hline $\mathrm{C}(014)-\mathrm{C}(01 \mathrm{~L})-\mathrm{C}(02 \mathrm{G})-\mathrm{N}(015)$ & $19.2(3)$ \\
\hline $\mathrm{C}(014)-\mathrm{C}(01 \mathrm{U})-\mathrm{C}(024)-\mathrm{N}(00 \mathrm{~W})$ & $-0.8(4)$ \\
\hline $\mathrm{C}(014)-\mathrm{C}(01 \mathrm{U})-\mathrm{C}(024)-\mathrm{C}(027)$ & $177.5(3)$ \\
\hline $\mathrm{C}(014)-\mathrm{C}(01 \mathrm{U})-\mathrm{C}(025)-\mathrm{C}(01 \mathrm{~W})$ & $-179.1(3)$ \\
\hline $\mathrm{C}(016)-\mathrm{O}(00 \mathrm{~F})-\mathrm{C}(02 \mathrm{Q})-\mathrm{C}(030)$ & $155.4(3)$ \\
\hline $\mathrm{C}(016)-\mathrm{C}(01 \mathrm{~F})-\mathrm{C}(01 \mathrm{M})-\mathrm{C}(019)$ & $-22.2(3)$ \\
\hline $\mathrm{C}(016)-\mathrm{C}(01 \mathrm{~F})-\mathrm{C}(01 \mathrm{M})-\mathrm{C}(01 \mathrm{Y})$ & $-139.8(2)$ \\
\hline $\mathrm{C}(016)-\mathrm{C}(01 \mathrm{~F})-\mathrm{C}(01 \mathrm{~N})-\mathrm{F}(003)$ & $-68.6(3)$ \\
\hline $\mathrm{C}(016)-\mathrm{C}(01 \mathrm{~F})-\mathrm{C}(01 \mathrm{~N})-\mathrm{F}(004)$ & $170.7(2)$ \\
\hline $\mathrm{C}(016)-\mathrm{C}(01 \mathrm{~F})-\mathrm{C}(01 \mathrm{~N})-\mathrm{F}(005)$ & $52.4(3)$ \\
\hline $\mathrm{C}(016)-\mathrm{C}(01 \mathrm{~F})-\mathrm{C}(02 \mathrm{~L})-\mathrm{N}(01 \mathrm{C})$ & $139.4(2)$ \\
\hline $\mathrm{C}(017)-\mathrm{N}(00 \mathrm{~V})-\mathrm{C}(02 \mathrm{~K})-\mathrm{C}(01 \mathrm{O})$ & $-4.2(4)$ \\
\hline $\mathrm{C}(017)-\mathrm{C}(01 \mathrm{E})-\mathrm{C}(01 \mathrm{O})-\mathrm{C}(01 \mathrm{G})$ & $-129.4(2)$ \\
\hline $\mathrm{C}(017)-\mathrm{C}(01 \mathrm{E})-\mathrm{C}(01 \mathrm{O})-\mathrm{C}(01 \mathrm{X})$ & 107.6(3) \\
\hline $\mathrm{C}(017)-\mathrm{C}(01 \mathrm{E})-\mathrm{C}(01 \mathrm{O})-\mathrm{C}(02 \mathrm{~K})$ & $-8.2(3)$ \\
\hline $\mathrm{C}(017)-\mathrm{C}(01 \mathrm{E})-\mathrm{C}(01 \mathrm{~V})-\mathrm{C}(01 \mathrm{~S})$ & $45.0(3)$ \\
\hline $\mathrm{C}(017)-\mathrm{C}(01 \mathrm{E})-\mathrm{C}(01 \mathrm{~V})-\mathrm{C}(020)$ & $-133.5(3)$ \\
\hline $\mathrm{C}(018)-\mathrm{C}(011)-\mathrm{C}(01 \mathrm{H})-\mathrm{N}(00 \mathrm{X})$ & $-3.5(4)$ \\
\hline $\mathrm{C}(018)-\mathrm{C}(011)-\mathrm{C}(01 \mathrm{H})-\mathrm{C}(01 \mathrm{Q})$ & $174.5(3)$ \\
\hline $\mathrm{C}(018)-\mathrm{C}(011)-\mathrm{C}(023)-\mathrm{C}(02 \mathrm{H})$ & $-177.6(3)$ \\
\hline $\mathrm{C}(018)-\mathrm{C}(01 \mathrm{R})-\mathrm{C}(021)-\mathrm{O}(00 \mathrm{~N})$ & $-66.9(3)$ \\
\hline $\mathrm{C}(018)-\mathrm{C}(01 \mathrm{R})-\mathrm{C}(021)-\mathrm{O}(012)$ & $110.5(4)$ \\
\hline $\mathrm{C}(018)-\mathrm{C}(01 \mathrm{R})-\mathrm{C}(02 \mathrm{~B})-\mathrm{F}(00 \mathrm{~B})$ & $57.8(4)$ \\
\hline $\mathrm{C}(018)-\mathrm{C}(01 \mathrm{R})-\mathrm{C}(02 \mathrm{~B})-\mathrm{F}(00 \mathrm{E})$ & $-62.3(3)$ \\
\hline $\mathrm{C}(018)-\mathrm{C}(01 \mathrm{R})-\mathrm{C}(02 \mathrm{~B})-\mathrm{F}(00 \mathrm{P})$ & 179.2(3) \\
\hline $\mathrm{C}(018)-\mathrm{C}(01 \mathrm{R})-\mathrm{C}(02 \mathrm{~N})-\mathrm{N}(010)$ & $12.9(3)$ \\
\hline $\mathrm{C}(019)-\mathrm{C}(01 \mathrm{~A})-\mathrm{C}(02 \mathrm{C})-\mathrm{C}(01 \mathrm{Z})$ & $-0.1(5)$ \\
\hline $\mathrm{C}(019)-\mathrm{C}(01 \mathrm{M})-\mathrm{C}(01 \mathrm{Y})-\mathrm{O}(00 \mathrm{G})$ & $67.3(3)$ \\
\hline $\mathrm{C}(019)-\mathrm{C}(01 \mathrm{M})-\mathrm{C}(01 \mathrm{Y})-\mathrm{N}(01 \mathrm{C})$ & $-109.4(3)$ \\
\hline $\mathrm{C}(019)-\mathrm{C}(022)-\mathrm{C}(028)-\mathrm{C}(01 \mathrm{Z})$ & $1.6(5)$ \\
\hline $\mathrm{C}(01 \mathrm{~A})-\mathrm{N}(00 \mathrm{U})-\mathrm{C}(01 \mathrm{D})-\mathrm{O}(007)$ & $-168.6(3)$ \\
\hline $\mathrm{C}(01 \mathrm{~A})-\mathrm{N}(00 \mathrm{U})-\mathrm{C}(01 \mathrm{D})-\mathrm{O}(00 \mathrm{O})$ & $12.2(5)$ \\
\hline $\mathrm{C}(01 \mathrm{~A})-\mathrm{C}(019)-\mathrm{C}(01 \mathrm{M})-\mathrm{C}(01 \mathrm{~F})$ & 106.3(3) \\
\hline $\mathrm{C}(01 \mathrm{~A})-\mathrm{C}(019)-\mathrm{C}(01 \mathrm{M})-\mathrm{C}(01 \mathrm{Y})$ & $-139.6(3)$ \\
\hline $\mathrm{C}(01 \mathrm{~A})-\mathrm{C}(019)-\mathrm{C}(022)-\mathrm{C}(028)$ & $-1.9(4)$ \\
\hline $\mathrm{C}(01 \mathrm{~B})-\mathrm{N}(010)-\mathrm{C}(02 \mathrm{~N})-\mathrm{C}(01 \mathrm{R})$ & $-8.5(4)$ \\
\hline $\mathrm{C}(01 \mathrm{~B})-\mathrm{C}(018)-\mathrm{C}(01 \mathrm{R})-\mathrm{C}(021)$ & $-133.0(2)$ \\
\hline $\mathrm{C}(01 \mathrm{~B})-\mathrm{C}(018)-\mathrm{C}(01 \mathrm{R})-\mathrm{C}(02 \mathrm{~B})$ & $103.9(3)$ \\
\hline
\end{tabular}




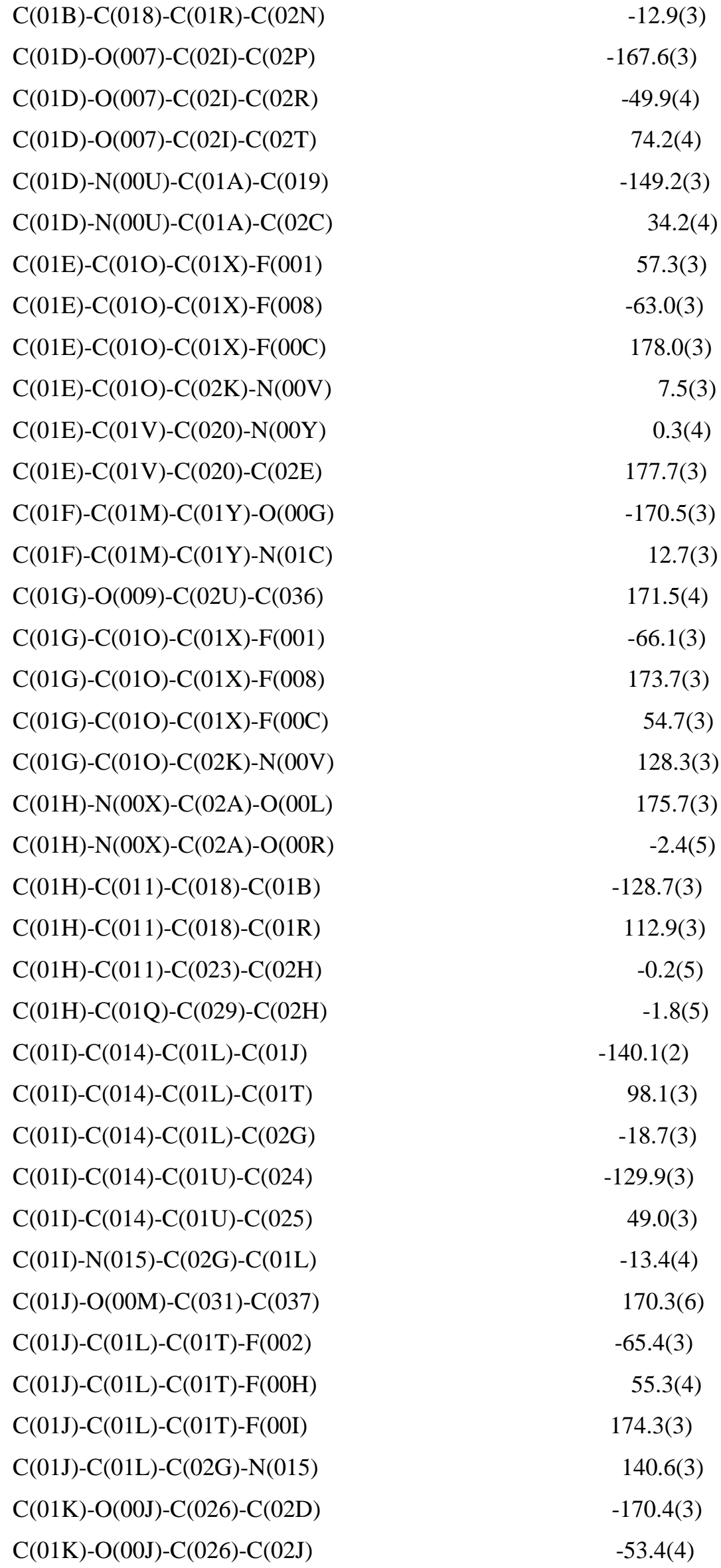




\begin{tabular}{|c|c|}
\hline $\mathrm{C}(01 \mathrm{~K})-\mathrm{O}(00 \mathrm{~J})-\mathrm{C}(026)-\mathrm{C}(02 \mathrm{~S})$ & $72.0(4)$ \\
\hline $\mathrm{C}(01 \mathrm{~K})-\mathrm{N}(00 \mathrm{Y})-\mathrm{C}(020)-\mathrm{C}(01 \mathrm{~V})$ & $-152.6(3)$ \\
\hline $\mathrm{C}(01 \mathrm{~K})-\mathrm{N}(00 \mathrm{Y})-\mathrm{C}(020)-\mathrm{C}(02 \mathrm{E})$ & $30.0(5)$ \\
\hline $\mathrm{C}(01 \mathrm{~L})-\mathrm{C}(014)-\mathrm{C}(01 \mathrm{I})-\mathrm{O}(00 \mathrm{D})$ & $-170.2(3)$ \\
\hline $\mathrm{C}(01 \mathrm{~L})-\mathrm{C}(014)-\mathrm{C}(01 \mathrm{I})-\mathrm{N}(015)$ & 11.6(3) \\
\hline $\mathrm{C}(01 \mathrm{~L})-\mathrm{C}(014)-\mathrm{C}(01 \mathrm{U})-\mathrm{C}(024)$ & $114.9(3)$ \\
\hline $\mathrm{C}(01 \mathrm{~L})-\mathrm{C}(014)-\mathrm{C}(01 \mathrm{U})-\mathrm{C}(025)$ & $-66.2(3)$ \\
\hline $\mathrm{C}(01 \mathrm{M})-\mathrm{C}(019)-\mathrm{C}(01 \mathrm{~A})-\mathrm{N}(00 \mathrm{U})$ & $2.6(4)$ \\
\hline $\mathrm{C}(01 \mathrm{M})-\mathrm{C}(019)-\mathrm{C}(01 \mathrm{~A})-\mathrm{C}(02 \mathrm{C})$ & $179.2(3)$ \\
\hline $\mathrm{C}(01 \mathrm{M})-\mathrm{C}(019)-\mathrm{C}(022)-\mathrm{C}(028)$ & $180.0(3)$ \\
\hline $\mathrm{C}(01 \mathrm{M})-\mathrm{C}(01 \mathrm{~F})-\mathrm{C}(01 \mathrm{~N})-\mathrm{F}(003)$ & $54.6(3)$ \\
\hline $\mathrm{C}(01 \mathrm{M})-\mathrm{C}(01 \mathrm{~F})-\mathrm{C}(01 \mathrm{~N})-\mathrm{F}(004)$ & $-66.1(3)$ \\
\hline $\mathrm{C}(01 \mathrm{M})-\mathrm{C}(01 \mathrm{~F})-\mathrm{C}(01 \mathrm{~N})-\mathrm{F}(005)$ & $175.6(2)$ \\
\hline $\mathrm{C}(01 \mathrm{M})-\mathrm{C}(01 \mathrm{~F})-\mathrm{C}(02 \mathrm{~L})-\mathrm{N}(01 \mathrm{C})$ & $18.1(3)$ \\
\hline $\mathrm{C}(01 \mathrm{~N})-\mathrm{C}(01 \mathrm{~F})-\mathrm{C}(01 \mathrm{M})-\mathrm{C}(019)$ & $-144.2(2)$ \\
\hline $\mathrm{C}(01 \mathrm{~N})-\mathrm{C}(01 \mathrm{~F})-\mathrm{C}(01 \mathrm{M})-\mathrm{C}(01 \mathrm{Y})$ & $98.3(2)$ \\
\hline $\mathrm{C}(01 \mathrm{~N})-\mathrm{C}(01 \mathrm{~F})-\mathrm{C}(02 \mathrm{~L})-\mathrm{N}(01 \mathrm{C})$ & $-99.3(3)$ \\
\hline $\mathrm{C}(01 \mathrm{O})-\mathrm{C}(01 \mathrm{E})-\mathrm{C}(01 \mathrm{~V})-\mathrm{C}(01 \mathrm{~S})$ & $-71.8(3)$ \\
\hline $\mathrm{C}(01 \mathrm{O})-\mathrm{C}(01 \mathrm{E})-\mathrm{C}(01 \mathrm{~V})-\mathrm{C}(020)$ & 109.7(3) \\
\hline $\mathrm{C}(01 \mathrm{P})-\mathrm{O}(00 \mathrm{Q})-\mathrm{C}(02 \mathrm{~V})-\mathrm{C}(033)$ & $66.7(5)$ \\
\hline $\mathrm{C}(01 \mathrm{P})-\mathrm{O}(00 \mathrm{Q})-\mathrm{C}(02 \mathrm{~V})-\mathrm{C}(034)$ & $-57.2(6)$ \\
\hline $\mathrm{C}(01 \mathrm{P})-\mathrm{O}(00 \mathrm{Q})-\mathrm{C}(02 \mathrm{~V})-\mathrm{C}(035)$ & $-174.1(4)$ \\
\hline $\mathrm{C}(01 \mathrm{P})-\mathrm{N}(00 \mathrm{~W})-\mathrm{C}(024)-\mathrm{C}(01 \mathrm{U})$ & $-149.5(3)$ \\
\hline $\mathrm{C}(01 \mathrm{P})-\mathrm{N}(00 \mathrm{~W})-\mathrm{C}(024)-\mathrm{C}(027)$ & $32.2(5)$ \\
\hline $\mathrm{C}(01 \mathrm{Q})-\mathrm{C}(029)-\mathrm{C}(02 \mathrm{H})-\mathrm{C}(023)$ & $-1.3(5)$ \\
\hline $\mathrm{C}(01 \mathrm{R})-\mathrm{C}(018)-\mathrm{C}(01 \mathrm{~B})-\mathrm{O}(00 \mathrm{~A})$ & $-172.6(2)$ \\
\hline $\mathrm{C}(01 \mathrm{R})-\mathrm{C}(018)-\mathrm{C}(01 \mathrm{~B})-\mathrm{N}(010)$ & $8.6(3)$ \\
\hline $\mathrm{C}(01 \mathrm{~S})-\mathrm{C}(01 \mathrm{~V})-\mathrm{C}(020)-\mathrm{N}(00 \mathrm{Y})$ & $-178.3(3)$ \\
\hline $\mathrm{C}(01 \mathrm{~S})-\mathrm{C}(01 \mathrm{~V})-\mathrm{C}(020)-\mathrm{C}(02 \mathrm{E})$ & $-0.9(5)$ \\
\hline $\mathrm{C}(01 \mathrm{~T})-\mathrm{C}(01 \mathrm{~L})-\mathrm{C}(02 \mathrm{G})-\mathrm{N}(015)$ & $-98.4(3)$ \\
\hline $\mathrm{C}(01 \mathrm{U})-\mathrm{C}(014)-\mathrm{C}(01 \mathrm{I})-\mathrm{O}(00 \mathrm{D})$ & $66.8(3)$ \\
\hline $\mathrm{C}(01 \mathrm{U})-\mathrm{C}(014)-\mathrm{C}(01 \mathrm{I})-\mathrm{N}(015)$ & $-111.4(3)$ \\
\hline $\mathrm{C}(01 \mathrm{U})-\mathrm{C}(014)-\mathrm{C}(01 \mathrm{~L})-\mathrm{C}(01 \mathrm{~J})$ & $-22.2(3)$ \\
\hline $\mathrm{C}(01 \mathrm{U})-\mathrm{C}(014)-\mathrm{C}(01 \mathrm{~L})-\mathrm{C}(01 \mathrm{~T})$ & $-143.9(3)$ \\
\hline $\mathrm{C}(01 \mathrm{U})-\mathrm{C}(014)-\mathrm{C}(01 \mathrm{~L})-\mathrm{C}(02 \mathrm{G})$ & $99.2(3)$ \\
\hline $\mathrm{C}(01 \mathrm{U})-\mathrm{C}(024)-\mathrm{C}(027)-\mathrm{C}(02 \mathrm{~F})$ & $2.3(5)$ \\
\hline $\mathrm{C}(01 \mathrm{~V})-\mathrm{C}(01 \mathrm{E})-\mathrm{C}(01 \mathrm{O})-\mathrm{C}(01 \mathrm{G})$ & $-9.3(3)$ \\
\hline $\mathrm{C}(01 \mathrm{~V})-\mathrm{C}(01 \mathrm{E})-\mathrm{C}(01 \mathrm{O})-\mathrm{C}(01 \mathrm{X})$ & $-132.3(3)$ \\
\hline
\end{tabular}




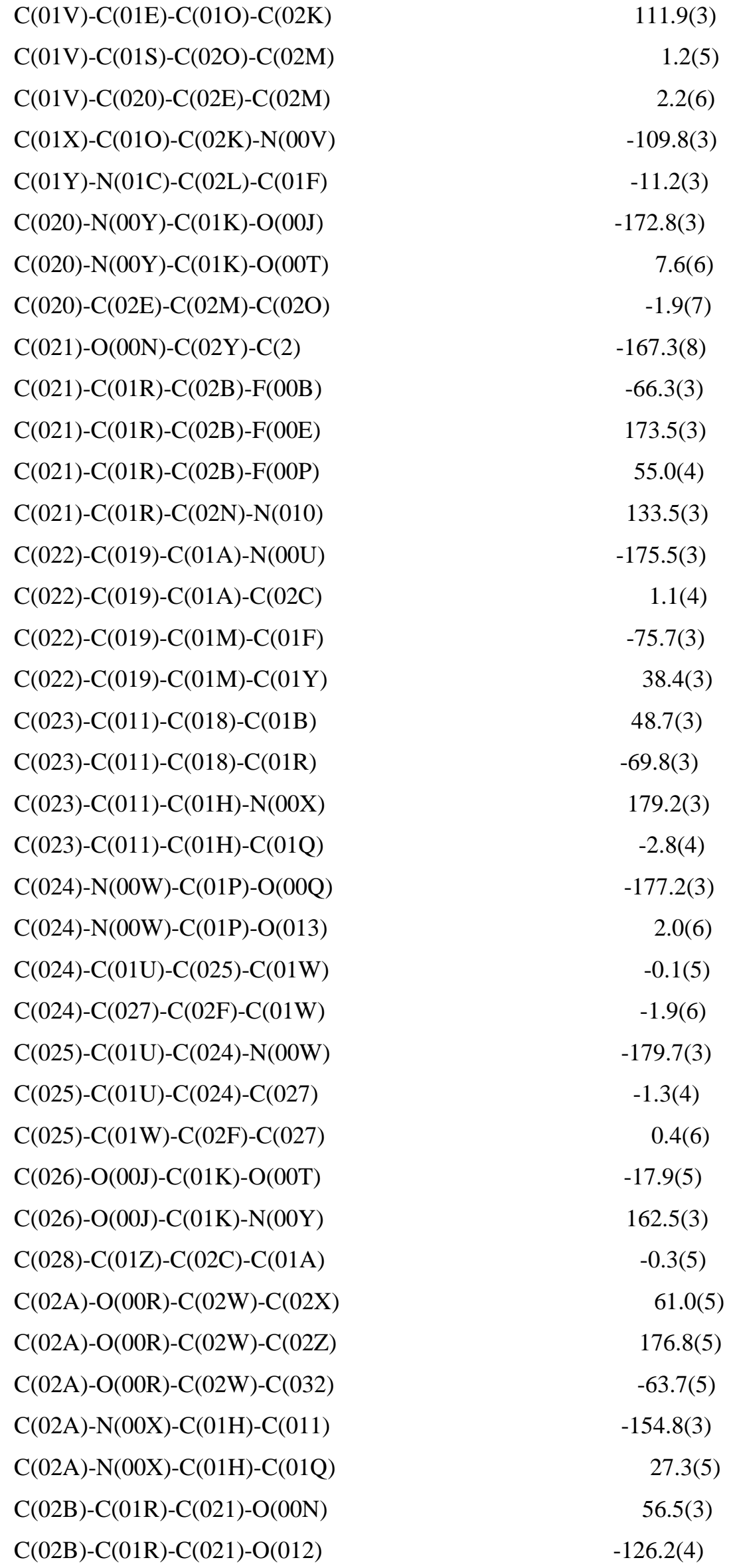




\begin{tabular}{|c|c|}
\hline $\mathrm{C}(02 \mathrm{~B})-\mathrm{C}(01 \mathrm{R})-\mathrm{C}(02 \mathrm{~N})-\mathrm{N}(010)$ & $-105.1(3)$ \\
\hline $\mathrm{C}(02 \mathrm{C})-\mathrm{C}(01 \mathrm{Z})-\mathrm{C}(028)-\mathrm{C}(022)$ & $-0.4(5)$ \\
\hline $\mathrm{C}(02 \mathrm{E})-\mathrm{C}(02 \mathrm{M})-\mathrm{C}(02 \mathrm{O})-\mathrm{C}(01 \mathrm{~S})$ & $0.1(6)$ \\
\hline $\mathrm{C}(02 \mathrm{~F})-\mathrm{C}(01 \mathrm{~W})-\mathrm{C}(025)-\mathrm{C}(01 \mathrm{U})$ & $0.6(5)$ \\
\hline $\mathrm{C}(02 \mathrm{G})-\mathrm{N}(015)-\mathrm{C}(01 \mathrm{I})-\mathrm{O}(00 \mathrm{D})$ & $-177.1(3)$ \\
\hline $\mathrm{C}(02 \mathrm{G})-\mathrm{N}(015)-\mathrm{C}(01 \mathrm{I})-\mathrm{C}(014)$ & $1.1(4)$ \\
\hline $\mathrm{C}(02 \mathrm{G})-\mathrm{C}(01 \mathrm{~L})-\mathrm{C}(01 \mathrm{~T})-\mathrm{F}(002)$ & $171.8(3)$ \\
\hline $\mathrm{C}(02 \mathrm{G})-\mathrm{C}(01 \mathrm{~L})-\mathrm{C}(01 \mathrm{~T})-\mathrm{F}(00 \mathrm{H})$ & $-67.5(3)$ \\
\hline $\mathrm{C}(02 \mathrm{G})-\mathrm{C}(01 \mathrm{~L})-\mathrm{C}(01 \mathrm{~T})-\mathrm{F}(00 \mathrm{I})$ & $51.5(3)$ \\
\hline $\mathrm{C}(02 \mathrm{I})-\mathrm{O}(007)-\mathrm{C}(01 \mathrm{D})-\mathrm{O}(00 \mathrm{O})$ & $-21.5(5)$ \\
\hline $\mathrm{C}(02 \mathrm{I})-\mathrm{O}(007)-\mathrm{C}(01 \mathrm{D})-\mathrm{N}(00 \mathrm{U})$ & 159.2(3) \\
\hline $\mathrm{C}(02 \mathrm{~K})-\mathrm{N}(00 \mathrm{~V})-\mathrm{C}(017)-\mathrm{O}(006)$ & $-178.2(3)$ \\
\hline $\mathrm{C}(02 \mathrm{~K})-\mathrm{N}(00 \mathrm{~V})-\mathrm{C}(017)-\mathrm{C}(01 \mathrm{E})$ & $-1.3(3)$ \\
\hline $\mathrm{C}(02 \mathrm{~K})-\mathrm{C}(01 \mathrm{O})-\mathrm{C}(01 \mathrm{X})-\mathrm{F}(001)$ & 171.2(3) \\
\hline $\mathrm{C}(02 \mathrm{~K})-\mathrm{C}(01 \mathrm{O})-\mathrm{C}(01 \mathrm{X})-\mathrm{F}(008)$ & $51.0(3)$ \\
\hline $\mathrm{C}(02 \mathrm{~K})-\mathrm{C}(01 \mathrm{O})-\mathrm{C}(01 \mathrm{X})-\mathrm{F}(00 \mathrm{C})$ & $-68.0(3)$ \\
\hline $\mathrm{C}(02 \mathrm{~L})-\mathrm{N}(01 \mathrm{C})-\mathrm{C}(01 \mathrm{Y})-\mathrm{O}(00 \mathrm{G})$ & $-177.9(3)$ \\
\hline $\mathrm{C}(02 \mathrm{~L})-\mathrm{N}(01 \mathrm{C})-\mathrm{C}(01 \mathrm{Y})-\mathrm{C}(01 \mathrm{M})$ & $-1.2(3)$ \\
\hline $\mathrm{C}(02 \mathrm{~L})-\mathrm{C}(01 \mathrm{~F})-\mathrm{C}(01 \mathrm{M})-\mathrm{C}(019)$ & $99.0(3)$ \\
\hline $\mathrm{C}(02 \mathrm{~L})-\mathrm{C}(01 \mathrm{~F})-\mathrm{C}(01 \mathrm{M})-\mathrm{C}(01 \mathrm{Y})$ & $-18.6(3)$ \\
\hline $\mathrm{C}(02 \mathrm{~L})-\mathrm{C}(01 \mathrm{~F})-\mathrm{C}(01 \mathrm{~N})-\mathrm{F}(003)$ & $168.8(3)$ \\
\hline $\mathrm{C}(02 \mathrm{~L})-\mathrm{C}(01 \mathrm{~F})-\mathrm{C}(01 \mathrm{~N})-\mathrm{F}(004)$ & $48.2(3)$ \\
\hline $\mathrm{C}(02 \mathrm{~L})-\mathrm{C}(01 \mathrm{~F})-\mathrm{C}(01 \mathrm{~N})-\mathrm{F}(005)$ & $-70.1(3)$ \\
\hline $\mathrm{C}(02 \mathrm{~N})-\mathrm{N}(010)-\mathrm{C}(01 \mathrm{~B})-\mathrm{O}(00 \mathrm{~A})$ & $-178.8(3)$ \\
\hline $\mathrm{C}(02 \mathrm{~N})-\mathrm{N}(010)-\mathrm{C}(01 \mathrm{~B})-\mathrm{C}(018)$ & $0.0(4)$ \\
\hline $\mathrm{C}(02 \mathrm{~N})-\mathrm{C}(01 \mathrm{R})-\mathrm{C}(021)-\mathrm{O}(00 \mathrm{~N})$ & 177.2(3) \\
\hline $\mathrm{C}(02 \mathrm{~N})-\mathrm{C}(01 \mathrm{R})-\mathrm{C}(021)-\mathrm{O}(012)$ & $-5.4(5)$ \\
\hline $\mathrm{C}(02 \mathrm{~N})-\mathrm{C}(01 \mathrm{R})-\mathrm{C}(02 \mathrm{~B})-\mathrm{F}(00 \mathrm{~B})$ & $171.7(3)$ \\
\hline $\mathrm{C}(02 \mathrm{~N})-\mathrm{C}(01 \mathrm{R})-\mathrm{C}(02 \mathrm{~B})-\mathrm{F}(00 \mathrm{E})$ & $51.5(3)$ \\
\hline $\mathrm{C}(02 \mathrm{~N})-\mathrm{C}(01 \mathrm{R})-\mathrm{C}(02 \mathrm{~B})-\mathrm{F}(00 \mathrm{P})$ & $-67.0(3)$ \\
\hline $\mathrm{C}(02 \mathrm{O})-\mathrm{C}(01 \mathrm{~S})-\mathrm{C}(01 \mathrm{~V})-\mathrm{C}(01 \mathrm{E})$ & $-179.4(3)$ \\
\hline $\mathrm{C}(02 \mathrm{O})-\mathrm{C}(01 \mathrm{~S})-\mathrm{C}(01 \mathrm{~V})-\mathrm{C}(020)$ & $-0.8(5)$ \\
\hline $\mathrm{C}(02 \mathrm{Q})-\mathrm{O}(00 \mathrm{~F})-\mathrm{C}(016)-\mathrm{O}(00 \mathrm{~K})$ & $-0.8(5)$ \\
\hline $\mathrm{C}(02 \mathrm{Q})-\mathrm{O}(00 \mathrm{~F})-\mathrm{C}(016)-\mathrm{C}(01 \mathrm{~F})$ & $176.6(3)$ \\
\hline $\mathrm{C}(02 \mathrm{U})-\mathrm{O}(009)-\mathrm{C}(01 \mathrm{G})-\mathrm{O}(00 \mathrm{~S})$ & $-6.7(5)$ \\
\hline $\mathrm{C}(02 \mathrm{U})-\mathrm{O}(009)-\mathrm{C}(01 \mathrm{G})-\mathrm{C}(01 \mathrm{O})$ & 171.3(3) \\
\hline $\mathrm{C}(02 \mathrm{~V})-\mathrm{O}(00 \mathrm{Q})-\mathrm{C}(01 \mathrm{P})-\mathrm{N}(00 \mathrm{~W})$ & $173.2(3)$ \\
\hline $\mathrm{C}(02 \mathrm{~V})-\mathrm{O}(00 \mathrm{Q})-\mathrm{C}(01 \mathrm{P})-\mathrm{O}(013)$ & $-6.1(6)$ \\
\hline
\end{tabular}




$$
\begin{aligned}
& \mathrm{C}(02 \mathrm{~W})-\mathrm{O}(00 \mathrm{R})-\mathrm{C}(02 \mathrm{~A})-\mathrm{O}(00 \mathrm{~L}) \\
& \mathrm{C}(02 \mathrm{~W})-\mathrm{O}(00 \mathrm{R})-\mathrm{C}(02 \mathrm{~A})-\mathrm{N}(00 \mathrm{X}) \\
& \mathrm{C}(02 \mathrm{Y})-\mathrm{O}(00 \mathrm{~N})-\mathrm{C}(021)-\mathrm{O}(012) \\
& \mathrm{C}(02 \mathrm{Y})-\mathrm{O}(00 \mathrm{~N})-\mathrm{C}(021)-\mathrm{C}(01 \mathrm{R}) \\
& \mathrm{C}(031)-\mathrm{O}(00 \mathrm{M})-\mathrm{C}(01 \mathrm{~J})-\mathrm{O}(00 \mathrm{Z}) \\
& \mathrm{C}(031)-\mathrm{O}(00 \mathrm{M})-\mathrm{C}(01 \mathrm{~J})-\mathrm{C}(01 \mathrm{~L})
\end{aligned}
$$

\begin{tabular}{|c|c|c|c|c|c|c|c|c|}
\hline \multicolumn{3}{|c|}{ D-H...A } & \multicolumn{2}{|r|}{$\mathrm{d}(\mathrm{D}-\mathrm{H})$} & \multirow{2}{*}{$\begin{array}{r}\mathrm{d}(\mathrm{H} \ldots \mathrm{A}) \\
0.86\end{array}$} & \multirow{2}{*}{$\begin{array}{r}\mathrm{d}(\mathrm{D} \ldots \mathrm{A}) \\
2.05\end{array}$} & \multicolumn{2}{|l|}{$<(\mathrm{DHA})$} \\
\hline NOOU & $--\mathrm{H} 00 \mathrm{U}$ &.. $\mathrm{O} 00 \mathrm{~A}$ & [ & $2656.04]$ & & & $2.9109(1)$ & 177 \\
\hline NOOV & $--\mathrm{H} 00 \mathrm{~V}$ &.. $\mathrm{O} 00 \mathrm{~A}$ & {[} & $1565.04]$ & 0.86 & 1.98 & $2.8220(1)$ & 16 \\
\hline N00W & --H00W &. .0006 & [ & {$\left[\begin{array}{ll}1555.01\end{array}\right]$} & 0.86 & 2.18 & $3.0281(1)$ & 16 \\
\hline NOOX & $--\mathrm{H} 00 \mathrm{X}$ &.. $\mathrm{O} 00 \mathrm{G}$ & [ & 2646.03] & 0.86 & 2.11 & $2.9596(1)$ & 169 \\
\hline NOOY & $--\mathrm{H} 00 \mathrm{Y}$ &.. $\mathrm{O} 00 \mathrm{D}$ & [ & $1555.02]$ & 0.86 & 2.17 & $3.0291(1)$ & 175 \\
\hline N010 & --H010 &. .0006 & & $1545.01]$ & 0.86 & 2.06 & $2.8902(1)$ & 163 \\
\hline N015 & --H015 &.. $\mathrm{O} 00 \mathrm{G}$ & & $1555.03]$ & 0.86 & 2.01 & $2.8485(1)$ & 16 \\
\hline N01C & $--\mathrm{H} 01 \mathrm{C}$ &.. $\mathrm{O} 00 \mathrm{D}$ & {[} & $1555.02]$ & 0.86 & 2.03 & $2.8501(1)$ & 160 \\
\hline $\mathrm{C} 02 \mathrm{~N}$ & $--\mathrm{H}$ &. .0012 & {[} & ] & 0.97 & 2.34 & $2.7946(1)$ & 108 \\
\hline C014 & --H014 & ..F002 & {[} & ] & 0.98 & 2.49 & $2.9189(1)$ & 106 \\
\hline C014 & --H014 &. .0006 & {[} & $1555.01]$ & 0.98 & 2.27 & $3.2405(1)$ & 169 \\
\hline C014 & --H014 & ..N00W & {[} & ] & 0.98 & 2.50 & $2.9369(1)$ & 107 \\
\hline C018 & --H018 &.. $\mathrm{F} 00 \mathrm{~B}$ & {[} & ] & 0.98 & 2.49 & $2.9123(1)$ & 106 \\
\hline $\mathrm{C} 018$ & --H018 &.. $\mathrm{N} 00 \mathrm{X}$ & {[} & ] & 0.98 & 2.52 & $2.9651(1)$ & 10 \\
\hline C018 & --H018 &.. $\mathrm{O} 00 \mathrm{G}$ & {[} & 2646.03] & 0.98 & 2.22 & $3.1867(1)$ & 170 \\
\hline C01E & $--\mathrm{H} 01 \mathrm{E}$ & ..F001 & {[} & ] & 0.98 & 2.51 & $2.9219(1)$ & 105 \\
\hline C01E & $--\mathrm{H} 01 \mathrm{E}$ &.. $\mathrm{O} 00 \mathrm{D}$ & {[} & $1555.02]$ & 0.98 & 2.22 & $3.1824(1)$ & 16 \\
\hline C01E & $--\mathrm{H} 01 \mathrm{E}$ & ..NOOY & {[} & ] & 0.98 & 2.49 & $2.9272(1)$ & 107 \\
\hline $\mathrm{C} 01 \mathrm{M}$ & --H01M &.. $\mathrm{F} 003$ & {[} & ] & 0.98 & 2.45 & $2.8930(1)$ & 10 \\
\hline $\mathrm{C} 01 \mathrm{M}$ & --H01M &.. $\mathrm{N} 00 \mathrm{U}$ & [ & ] & 0.98 & 2.52 & $2.9293(1)$ & 105 \\
\hline $\mathrm{C} 01 \mathrm{M}$ & --H01M &.. $\mathrm{O} 00 \mathrm{~A}$ & [ & {$\left[\begin{array}{ll}2656.04\end{array}\right]$} & 0.98 & 2.27 & $3.2310(1)$ & 166 \\
\hline C01Q & --H01Q &.. $\mathrm{O} 00 \mathrm{R}$ & {[} & ] & 0.93 & 2.27 & $2.7854(1)$ & 11 \\
\hline $\mathrm{C} 027$ & --H027 &. .0013 & {[} & ] & 0.93 & 2.36 & $2.8421(1)$ & 112 \\
\hline $\mathrm{C} 02 \mathrm{C}$ & $--\mathrm{H} 02 \mathrm{~T}$ &.. $\mathrm{O} 00 \mathrm{O}$ & {[} & ] & 0.93 & 2.44 & $2.9045(1)$ & 111 \\
\hline $\mathrm{C} 02 \mathrm{E}$ & $--\mathrm{H} 02 \mathrm{E}$ &.. $\mathrm{O} 00 \mathrm{~T}$ & {[} & ] & 0.93 & 2.36 & $2.8529(1)$ & 113 \\
\hline $\mathrm{C} 02 \mathrm{G}$ & $--\mathrm{H} 02 \mathrm{~S}$ &.. $\mathrm{O} 00 \mathrm{Z}$ & {[} & ] & 0.97 & 2.41 & $2.8164(1)$ & 104 \\
\hline $\mathrm{C} 02 \mathrm{~J}$ & $--\mathrm{H} 02 \mathrm{~F}$ &.. $\mathrm{O} 00 \mathrm{Z}$ & {[} & 2546.02] & 0.96 & 2.43 & $3.3357(1)$ & 158 \\
\hline
\end{tabular}

Symmetry transformations used to generate equivalent atoms:

Table 7. Hydrogen bonds for $3 a$ [A and deg.]. 


\begin{tabular}{|c|c|c|c|c|c|c|c|}
\hline $\mathrm{CO2J}$ & $--\mathrm{H} 02 \mathrm{G}$ &.. $\mathrm{O} 00 \mathrm{~T}$ & {[} & ] & 0.96 & 2.39 & $2.9539(1)$ \\
\hline $\mathrm{C} 02 \mathrm{~K}$ & --H02I &.. $\mathrm{O} 00 \mathrm{~S}$ & {[} & ] & 0.97 & 2.36 & $2.8199(1)$ \\
\hline $\mathrm{C} 02 \mathrm{~L}$ & $--\mathrm{H} 02 \mathrm{~V}$ &.. $\mathrm{O} 00 \mathrm{~K}$ & {[} & ] & 0.97 & 2.37 & $2.8041(1)$ \\
\hline $\mathrm{C} 02 \mathrm{Q}$ & --H02Z &.. $\mathrm{O} 00 \mathrm{Z}$ & [ & $1545.02]$ & 0.97 & 2.53 & $3.3623(1)$ \\
\hline $\mathrm{C} 02 \mathrm{R}$ & $--\mathrm{Hb}$ &. .0012 & {[} & $1556.04]$ & 0.96 & 2.46 & $3.3990(1)$ \\
\hline $\mathrm{C} 02 \mathrm{R}$ & $--\mathrm{Hc}$ &.. $\mathrm{O} 00 \mathrm{O}$ & {[} & ] & 0.96 & 2.43 & $2.9428(1)$ \\
\hline $\mathrm{C} 02 \mathrm{~S}$ & $--\mathrm{H} 02 \mathrm{~J}$ &.. $\mathrm{O} 00 \mathrm{~T}$ & {[} & ] & 0.96 & 2.46 & $3.0120(1)$ \\
\hline $\mathrm{C} 02 \mathrm{~T}$ & --H0AA &.. $\mathrm{O} 00 \mathrm{O}$ & {[} & ] & 0.96 & 2.48 & $3.0369(1)$ \\
\hline $\mathrm{C} 02 \mathrm{U}$ & $--\mathrm{H} 02 \mathrm{~N}$ &. .0012 & {[} & $1555.04]$ & 0.97 & 2.57 & $3.3889(1)$ \\
\hline $\mathrm{C} 02 \mathrm{X}$ & --H3AA &.. $\mathrm{O} 00 \mathrm{~L}$ & {[} & ] & 0.96 & 2.46 & $3.0078(1)$ \\
\hline C031 & --H03E &.. $\mathrm{O} 00 \mathrm{~K}$ & {[} & $1565.03]$ & 0.97 & 2.58 & $3.5225(1)$ \\
\hline $\mathrm{C} 032$ & $--\mathrm{H} 03 \mathrm{~W}$ &.. $\mathrm{O} 00 \mathrm{~L}$ & {[} & ] & 0.96 & 2.40 & $2.9919(1)$ \\
\hline C033 & $--\mathrm{H} 03 \mathrm{~F}$ &. .0013 & {[} & ] & 0.96 & 2.52 & $3.0335(1)$ \\
\hline $\mathrm{C} 034$ & --H03K & .0013 & {[} & ] & 0.96 & 2.41 & $2.9479(1)$ \\
\hline
\end{tabular}

